\title{
Numerical Prediction of the Interference Drag of a Streamlined Strut Intersecting a Surface in Transonic Flow
}

\author{
Philippe-André Tétrault \\ Dissertation submitted to the Faculty of the \\ Virginia Polytechnic Institute and State University \\ in partial fulfillment of the requirements for the degree of
}

Doctor of Philosophy

in

Aerospace Engineering

Joseph A. Schetz, Chair

Bernard Grossman

Rakesh K. Kapania

William H. Mason

Pradeep Raj

January 31, 2000

Blacksburg, Virginia

Keywords: Interference Drag, Strut-Braced Wing, Junction Flow, Transonic Flow Copyright @2000, Philippe-André Tétrault 


\title{
Numerical Prediction of the Interference Drag of a Streamlined Strut Intersecting a Surface in Transonic Flow
}

\author{
by \\ Philippe-André Tétrault \\ Committee Chairman: Joseph A. Schetz \\ Aerospace Engineering
}

(ABSTRACT)

In transonic flow, the aerodynamic interference that occurs on a strut-braced wing airplane, pylons, and other applications is significant. The purpose of this work is to provide relationships to estimate the interference drag of wing-strut, wing-pylon, and wing-body arrangements. Those equations are obtained by fitting a curve to the results obtained from numerous Computational Fluid Dynamics (CFD) calculations using state-of-the-art codes that employ the Spalart-Allmaras turbulence model.

In order to estimate the effect of the strut thickness, the Reynolds number of the flow, and the angle made by the strut with an adjacent surface, inviscid and viscous calculations are performed on a symmetrical strut at an angle between parallel walls. The computations are conducted at a Mach number of 0.85 and Reynolds numbers of 5.3 and 10.6 million based on the strut chord. The interference drag is calculated as the drag increment of the arrangement compared to an equivalent two-dimensional strut of the same cross-section. The results show a rapid increase of the interference drag as the angle of the strut deviates from a position perpendicular to the wall. Separation regions appear for low intersection angles, but the viscosity generally provides a positive effect in alleviating the strength of the shock near the junction and thus the drag penalty. When the thickness-to-chord ratio of the strut is reduced, the flowfield is disturbed only locally at the intersection of the strut with the wall. This study provides an equation to estimate the interference drag of simple intersections in transonic flow.

In the course of performing the calculations associated with this work, an unstructured flow solver was utilized. Accurate drag prediction requires a very fine grid and this leads to problems associated with the grid generator. Several challenges facing the unstructured 
grid methodology are discussed: slivers, grid refinement near the leading edge and at the trailing edge, grid convergence studies, volume grid generation, and other practical matters concerning such calculations.

This work was sponsored by NASA Langley Research Center, Grant No. NAG1-1852. Partial financial support for the author was also provided by Fonds FCAR, Fonds pour la Formation de Chercheurs et l'Aide à la Recherche, Governement of Québec, Canada. 


\title{
Dedication
}

\author{
In Memoriam \\ Denis Goyette \\ 1940-1998 \\ In hope that his family and grandchildren will live in great happiness
}

To the most important people in my life:

my parents, Marie-Claude and Guy-Paul, who care so much about my sister and I, my beloved sister, Rose-Marie, and my sunshine, Annick 


\section{Acknowledgments}

First and foremost, I would like to thank my family for always supporting me through good and difficult times over the last three years. My parents and my sister have encouraged me to pursue my studies despite the obstacles on the road. My girlfriend Annick has always been there for me during the last year. She has brought a lot of peace to my soul. I love all of you very much.

This work wouldn't have been possible without the help of the chairman of my committee, Dr. Schetz. Despite the difficulties we faced in the research, he was always there to remind me that I was doing good work. I have always had the feeling that he enjoys what he is doing. Dr. Mason loves to teach and that's what explains why I have spent so much time in his office, leaving with more answers than questions. I also appreciate the fact that he has always had a friendly attitude towards me. I have enjoyed interacting with Dr. Kapania, whose cheerful attitude and perpective on life and research is very similar to mine. I would like to acknowledge the contribution of Dr. Grossman to the team in the field of numerical calculations and his desire of improving the life of the graduate students by forming the Graduate Advisory Committee. Dr. Raj deserves a lot of thanks for providing me with useful advice throughout this project despite his busy schedule. I appreciate the fact that he has always been interested in knowing more about the evolution of my research work and career. The "virtual" presence of Dr. Haftka during our weekly meetings has been very beneficial because of his ability to foresee difficulties and to get the team members to not lose sight of the big picture.

I owe a lot to Dr. Anderson from NASA Langley for his help in solving some of the problems I faced with his flow solver. Without any doubt, he taught me more about CFD than anyone else. Through numerous interactions via phone and e-mail, I think we have become partners and, ultimately, friends. I am indebted to Mr. Asbury from NASA Langley who provided me access to the computing facilities at NASA and arranged two trips for me 
there. His interest in the project has been obvious throughout the duration of the contract. I would like to thank Dr. Pirzadeh and Dr. Parikh from NASA Langley for their help in grid generation and flow visualization. I am very grateful to Dr. Devenport who gave me an excellent preparation for the Ph.D. prelims. Last fall, Dr. Cliff was kind enough to allow me to work temporarily at ICAM and this is what explains why I am completing my degree earlier than I anticipated. I would also like to mention that Mr. Greenfield, the system administrator, was a dream come true: he cared about my work and really did everything he could to help me achieve my goal.

The dream of pursuing and completing graduate studies abroad wouldn't have been possible without the constant guidance of my mentor, Dr. Pelletier from École Polytechnique. Dr. Pelletier has the incredible ability of looking at life and things with a refreshing perspective. I am also grateful to Mr. Godin who has set an example for me to follow. I know both of them are proud of me right now.

The TBW team was a very nice environment to work in because of Amir, Andy, Frank, Erwin, Joel, Jay, and Mike. To me, Andy and Frank go hand in hand because they share the same sense of humor, strong work ethic, and an insatiable desire to do their best in whatever they attempt to do. Life is so much better with them around. Despite all the financial, professional and personal challenges Erwin has faced since he joined Virginia Tech, he has kept his big smile and it has been a pleasure to work with him. I wish to recognize Joel's contribution to the TBW team as well. He created the framework of the MDO code and was an eye-opener with his cleverness and engineering judgment.

If friendship is a garden, I believe I own the most beautiful garden of all. My officemate and very good friend Amir has been through a lot on the TBW team and I wish him well in his career. He has been an inspiration for me for never giving up and for constantly trying to please everyone. Bragi, Stina, and their son Martin Oli are extraordinary people whose generosity and friendship go beyond anything I have experienced. The bond we have together will last a lifetime. Last but not least, I would like to thank Mohamed for the energy he brought to all of us and to me in particular. His intensity and his desire to always reach for the best are amazing. I miss his laugh and his personality very much.

I wish to acknowledge the rest of my "international" friends: Ohh, Tanja, Esa, Jesper, Sampsa, Tomas, and Jean-Pierre and his family who took very good care of me during their stay in Blacksburg. I wish to thank the following people as well: Serhat, Semere, David, 
Lance, Chittiappa, Sam, Troy, Samy, Dan, Chuck, Patrick, Mike, Matt, John, Cindy, Kevin, Alejandro, Iossif, Dave and Andrea Cohen, and the others. And to Ms. Foushee, Ms. Coe, and Ms. Williams, a big thanks also.

Without the help of my friend Frédéric, I wouldn't be heading to Pratt \& Whitney after obtaining my degree. And finally, who would dare to drive 14 hours to come visit their friend in Blacksburg? My best friends Martine and Bruno did just that numerous times and brought with them the most beautiful present: their son Jérémy, who is now my godson. 


\section{Contents}

1 Introduction $\quad 1$

2 Literature Review $\quad 3$

2.1 Innovative Aircraft Concepts . . . . . . . . . . . . . . . . . . . 3

2.2 Interference Drag . . . . . . . . . . . . . . . . . . . . . . 5

2.2.1 Subsonic Flow . . . . . . . . . . . . . . . . . . 6

2.2.2 Transonic Flow . . . . . . . . . . . . . . . . . . . . . . 10

2.2.3 Supersonic Flow . . . . . . . . . . . . . . . . . . . . . . 15

2.3 Contributions to the Field . . . . . . . . . . . . . . . . . . . . . 17

3 Description of the Computational Fluid Dynamics Tools 18

3.1 Unstructured Grid Generation . . . . . . . . . . . . . . . . . . 19

3.1.1 Two-Dimensional Grids .................... 19

3.1 .2 Three-Dimensional Grids . . . . . . . . . . . . . . . . . . . . 21

3.2 Flow Solver . . . . . . . . . . . . . . . . . . . . . . 28

3.2.1 Governing Equations . . . . . . . . . . . . . . . . 28

3.2.2 Turbulence Model . . . . . . . . . . . . . . . . . . . . 31

3.2.3 Finite Volume Formulation . . . . . . . . . . . . . . . . . . . 32

3.2.4 Boundary Conditions . . . . . . . . . . . . . . 38 
3.2 .5 Time Integration $\ldots \ldots \ldots \ldots$

3.2 .6 Multigrid . . . . . . . . . . . . . . . . . . 42

\section{Aerodynamic Analysis of a Strut-Braced Wing Aircraft}

Using the Euler Equations

4.1 Approach . . . . . . . . . . . . . . . . . . . . . 43

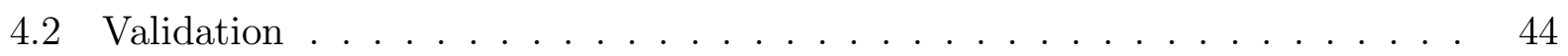

4.3 Description of the Airplane . . . . . . . . . . . . . . . . . . . . . 46

4.4 Analysis of the Wing Alone . . . . . . . . . . . . . . . . 48

4.5 Analysis of the Strut-Braced Wing . . . . . . . . . . . 54

4.6 Concluding Remarks . . . . . . . . . . . . . . . . . . . . 62

5 Analysis of the Interference Drag of a Strut Inclined to a Surface Using $\begin{array}{ll}\text { Euler and Navier-Stokes Equations } & 64\end{array}$

5.1 Modeling of the Problem . . . . . . . . . . . . . . . . . . . . 65

5.2 Validation . . . . . . . . . . . . . . . . . . . 65

5.3 Problem Description $\ldots \ldots \ldots \ldots \ldots \ldots \ldots \ldots$

5.4 Calculation of the Interference Drag . . . . . . . . . . . . . . . 69

5.5 Results and Discussion . . . . . . . . . . . . . . . 71

$5.5 .1 \quad$ Euler Calculations . . . . . . . . . . . . . . . . . 72

$5.5 .2 \quad$ Navier-Stokes Calculations . . . . . . . . . . . . . . . . . 77

5.6 Flowfield Details . . . . . . . . . . . . . . . . . . . . . . . . 89

5.6.1 Flowfield Around the NACA 64A005 Strut Section . . . . . . . 90

5.6.2 Flowfield Around the NACA 64A007.5 Strut Section _. . . . . . . 94

5.6 .3 Two-Dimensionality of the Flow . . . . . . . . . . . . . . 100 
6 Challenges in Computational Fluid Dynamics

6.1 Trailing edge closure . . . . . . . . . . . . . . . . . . . . . . . . 104

6.2 Grid convergence studies . . . . . . . . . . . . . . . . . . . . . 110

6.3 Grid generation in the vicinity of the leading edge and the trailing edge . . . 112

6.4 Impossibility to Close the Volume Grid . . . . . . . . . . . . . . . . 119

6.5 Slivers . . . . . . . . . . . . . . . . . . . . . . . . 122

7 Conclusion 


\section{List of Figures}

3.1 Flowchart of the grid generation process in three dimensions . . . . . . . . 22

3.2 Patch definition on a wing planform (a) View of the planform (b) Close-up view of the wing tip . . . . . . . . . . . . . . . . . 23

3.3 Five linear sources located inside the wing . . . . . . . . . . . . . . . 24

3.4 Wing and nodal sources located at the corners of the computational box . . 25

3.5 Influence of the normals on the growth of the viscous layers around a sharp corner (a) Addition of normals (AFLR2) (b) Use of existing normals only (VGRIDns) 27

3.6 One of three individual control volumes within a triangular cell . . . . . . . 33

3.7 One of four individual control volumes within a tetrahedral cell . . . . . . 33

3.8 Control volume obtained by combining the individual control volumes of the neighboring cells . . . . . . . . . . . . . . . . . . . . . 34

3.9 Control volume and the associated surface normals . . . . . . . . . . . 36

4.1 Surface mesh on the ONERA M6 wing (a) Wing surface and symmetry plane (b) Triangulation of the symmetry plane . . . . . . . . . . . . . 45

4.2 Mach number contours on the ONERA M6 wing surface . . . . . . . . . 45

4.3 Pressure distribution on the ONERA M6 wing (a) $\eta=20 \%$ (b) $\eta=44 \%$ (c) $\eta=65 \%$ (d) $\eta=80 \%$ (e) $\eta=90 \%$ (f) $\eta=95 \%$ (g) $\eta=99 \% \ldots 47$

4.4 Wing twist distribution for the strut-braced wing $\left(C_{L}=0.5191\right) \ldots 49$

4.5 Surface grid on the SS5 wing (a) Wing upper surface (b) Wing lower surface 49 
4.6 Convergence history, $M_{\infty}=0.85, \alpha_{\text {root }}=2.36^{\circ}\left(\alpha=-3.97^{\circ}\right)$ (a) Lift coefficient and residual (b) Drag coefficient and residual . . . . . . . . . 50

4.7 Mesh on the wing upper surface (a) Very coarse grid (b) Coarse grid (c) Medium grid (d) Fine grid . . . . . . . . . . . . . . . . . . . . . 52

4.8 Effect of the grid refinement on the lift and drag coefficients for the wing alone, $M_{\infty}=0.85, \alpha_{\text {root }}=2.36^{\circ}\left(\alpha=-3.97^{\circ}\right)$ (a) Variation of the lift coefficient (b) Variation of the drag coefficient ...............

4.9 Mach number contours on the SS5 clean wing, $M_{\infty}=0.85, \alpha_{\text {root }}=2.36^{\circ}$ $\left(\alpha=-3.97^{\circ}\right)$ (a) Wing upper surface (b) Wing lower surface . . . . . . . .

4.10 Influence of the strut on the spanload distribution of the SS5 strut-braced wing design, $M_{\infty}=0.85, \alpha_{\text {root }}=2.36^{\circ}\left(\alpha=-3.97^{\circ}\right) \ldots \ldots \ldots$

4.11 Effect of the grid refinement on the pressure distribution for the wing alone, $M_{\infty}=0.85, \alpha_{\text {root }}=2.36^{\circ}\left(\alpha=-3.97^{\circ}\right)$ (a) $\eta=0 \%$ (b) $\eta=25 \%$ (c) $\eta=50 \%$ (d) $\eta=70 \%$ (e) $\eta=75 \%$ (f) $\eta=80 \%$ (g) $\eta=85 \%$ (h) $\eta=90 \%$ (i) $\eta=95 \% \ldots \ldots \ldots \ldots \ldots \ldots$

4.12 Strut-braced wing designs (a) With straight strut (b) With arch-shaped strut 56

4.13 Surface grid on the SS5 strut-braced wing with a straight strut (a) Wing upper surface (b) Wing and strut lower surfaces (c) Close-up view of the wing-strut

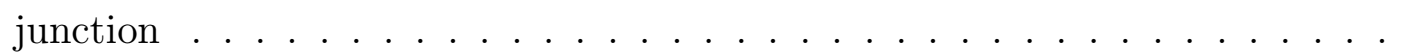

4.14 Mach number contours on the SS5 strut-braced wing with a straight strut, $M_{\infty}=0.85, \alpha_{\text {root }}=2.36^{\circ}\left(\alpha=-3.97^{\circ}\right)$ (a) Wing upper surface (b) Wing lower surface (the strut has been removed from the figure to show the contours on the wing surface) $\ldots \ldots \ldots \ldots \ldots$

4.15 Mach number contours on the SS5 strut-braced wing with a 4-ft arch, $M_{\infty}=0.85$, $\alpha_{\text {root }}=2.36^{\circ}\left(\alpha=-3.97^{\circ}\right)$ (a) Wing upper surface (b) Wing lower surface (the strut has been removed from the figure to show the contours on the wing

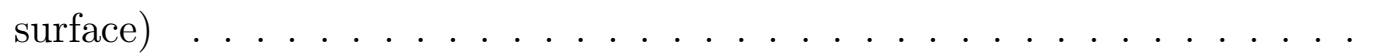


4.16 Influence of the strut on the wing pressure distribution for the SS5 strutbraced wing design, $M_{\infty}=0.85, \alpha_{\text {root }}=2.36^{\circ}\left(\alpha=-3.97^{\circ}\right)$ (a) $\eta=0 \%$ (b) $\eta=25 \%$ (c) $\eta=50 \%$ (d) $\eta=70 \%$ (e) $\eta=75 \%$ (f) $\eta=80 \%$ (g) $\eta=85 \%$ (h) $\eta=90 \%$ (i) $\eta=95 \% \ldots \ldots \ldots 60 \ldots \ldots$

4.17 Pressure distribution on the strut of the SS5 strut-braced wing design, $M_{\infty}=0.85$, $\alpha_{\text {root }}=2.36^{\circ}\left(\alpha=-3.97^{\circ}\right)$ (a) $\eta=25 \%$ (b) $\eta=50 \%$ (c) $\eta=70 \%$ (d) $\eta=75 \%$ (e) $\eta=80 \%$ (f) $\eta=85 \% \ldots \ldots$

4.18 Interference drag penalty $\Delta C_{D}$ in terms of the arch radius, $M_{\infty}=0.85$, $\alpha_{\text {root }}=2.36^{\circ} \ldots \ldots \ldots \ldots \ldots \ldots \ldots \ldots \ldots \ldots \ldots \ldots \ldots \ldots \ldots$

$5.1 C_{p}$ distribution for the AGARD test case, $M_{\infty}=0.73, \alpha=1.5^{\circ}, \operatorname{Re}_{c}=6 \times$ $10^{6}$ (a) y $=2 \mathrm{~mm}(\eta=0.6 \%)(\mathrm{b}) \mathrm{y}=10 \mathrm{~mm}(\eta=2.9 \%)(\mathrm{c}) \mathrm{y}=20 \mathrm{~mm}$ $(\eta=5.9 \%)(\mathrm{d}) \mathrm{y}=40 \mathrm{~mm}(\eta=11.8 \%)(\mathrm{e}) \mathrm{y}=80 \mathrm{~mm}(\eta=23.5 \%)$ (f) $\mathrm{y}=170 \mathrm{~mm}(\eta=50.0 \%) \quad \ldots \ldots \ldots \ldots$

5.2 Strut between parallel walls in transonic flow . . . . . . . . . . . . . . 69

5.3 Convergence History NACA 64A007.5 - $\phi=30^{\circ}, M_{\infty}=0.85, \alpha=0^{\circ}$ (Euler solution) - Residual (left axis) and total drag coefficient (right axis) .

$5.4 C_{p}$ distribution for the NACA 64A005 strut, $M_{\infty}=0.85, \alpha=0^{\circ}$ (Euler solution) (a) $\eta=0 \%$ (b) $\eta=10 \%$ (c) $\eta=20 \%$ (d) $\eta=30 \%$ (e) $\eta=40 \%$ (f) $\eta=50 \%$

$5.5 C_{p}$ distribution for the NACA 64A007.5 strut, $M_{\infty}=0.85, \alpha=0^{\circ}$ (Euler solution) (a) $\eta=0 \%$ (b) $\eta=10 \%$ (c) $\eta=20 \%$ (d) $\eta=30 \%$ (e) $\eta=40 \%$

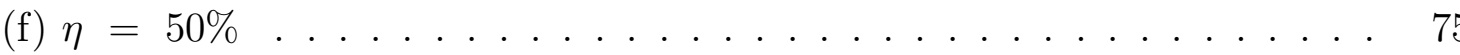

5.6 Spanload distribution, $M_{\infty}=0.85, \alpha=0^{\circ}$ (Euler solution) (a) NACA 64A005 (b) NACA $64 \mathrm{~A} 007.5 \ldots \ldots \ldots \ldots \ldots \ldots$

5.7 Variation of $y^{+}$for the first grid point from the NACA 64A007.5 airfoil surface, $M_{\infty}=0.85, \alpha=0^{o}, R e_{c}=10.6 \times 10^{6}$ (Navier-Stokes solution) . . . . 78

5.8 Fine viscous surface grid at the end plane of the NACA 64A007.5 strut -

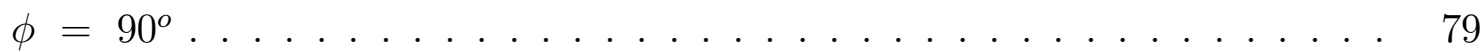


5.9 Upper surface triangulation of the NACA $64 \mathrm{~A} 007.5$ strut $-\phi=90^{\circ}$ (inviscid side walls $\ldots \ldots \ldots \ldots \ldots$. . . . . . . . . . . . . . . . 80

5.10 Effects of grid refinement on $C_{p}$ distribution for the NACA 64A007.5 strut $\phi=90^{\circ}, M_{\infty}=0.85, \alpha=0^{\circ}, R e_{c}=5.3 \times 10^{6}$ (Navier-Stokes solution) (a) $\eta=0 \%$ (b) $\eta=10 \%$ (c) $\eta=20 \%$ (d) $\eta=30 \%$ (e) $\eta=40 \%$

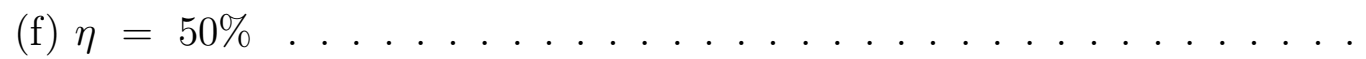

5.11 Grid convergence study, $M_{\infty}=0.85, \alpha=0^{\circ}, R e_{c}=5.3 \times 10^{6}$ (NavierStokes solution) - Pressure and skin friction drag components (left axis) and total drag coefficient (right axis) $\ldots \ldots \ldots \ldots \ldots \ldots$

5.12 Convergence history for the NACA 64A007.5 strut $-\phi=30^{\circ}, M_{\infty}=0.85$, $\alpha=0^{\circ}, R e_{c}=5.3 \times 10^{6}$ (Navier-Stokes solution) - Residual (left axis) and components of the drag coefficient (right axis) . . . . . . . . . . .

$5.13 C_{p}$ distribution for the NACA $64 \mathrm{~A} 005$ strut, $M_{\infty}=0.85, \alpha=0^{\circ}$, $R e_{c}=5.3 \times 10^{6}$ (Navier-Stokes solution) (a) $\eta=0 \%$ (b) $\eta=10 \%$ (c) $\eta=20 \%$ (d) $\eta=30 \%$ (e) $\eta=40 \%$ (f) $\eta=50 \% \ldots \ldots$

$5.14 C_{p}$ distribution for the NACA 64A007.5 strut, $M_{\infty}=0.85, \alpha=0^{\circ}$, $R e_{c}=5.3 \times 10^{6}$ (Navier-Stokes solution) (a) $\eta=0 \%$ (b) $\eta=10 \%$ (c) $\eta=20 \%$ (d) $\eta=30 \%$ (e) $\eta=40 \%$ (f) $\eta=50 \% \ldots \ldots$. . . .

5.15 Spanload distribution, $M_{\infty}=0.85, \alpha=0^{\circ}$ (Navier-Stokes solution) (a) NACA 64A005 with $R e_{c}=5.3 \times 10^{6}$ (b) NACA 64A007.5 with $R e_{c}=5.3 \times$ $10^{6}$ (c) NACA 64A005 with $R e_{c}=10.6 \times 10^{6}$ (d) NACA 64A007.5 with

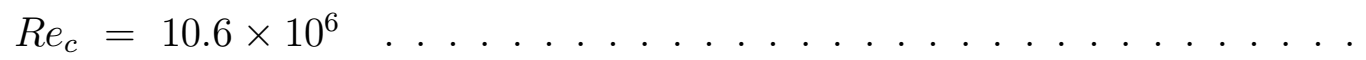

5.16 Response surface with contours of $C_{D_{\text {interf }}}$ for $R e_{c}=5.3 \times 10^{6} \ldots \ldots 89$

5.17 Comparison of the response surface with the CFD data for $M_{\infty}=0.85$, $\alpha=0^{\circ}$, and $R e_{c}=5.3 \times 10^{6}$ and $10.6 \times 10^{6} \ldots \ldots \ldots \ldots$

5.18 Pressure contours with streamtraces at $\eta=0.0002 \%$ for NACA 64A005, $M_{\infty}=0.85, \alpha=0^{\circ}, R e_{c}=5.3 \times 10^{6}-$ Euler solution: (a) $\phi=90^{\circ}$ (b) $\phi=60^{\circ}$ (c) $\phi=30^{\circ}$ - Navier-Stokes solution: (d) $\phi=90^{\circ}$ (inviscid side walls) (e) $\phi=90^{\circ}$ (f) $\phi=60^{\circ}$ (g) $\phi=30^{\circ} \ldots \ldots \ldots$ 
5.19 Pressure contours with streamtraces at $\eta=10 \%$ for NACA $64 \mathrm{~A} 005, M_{\infty}=0.85$, $\alpha=0^{\circ}, R e_{c}=5.3 \times 10^{6}$ - Euler solution: (a) $\phi=90^{\circ}$ (b) $\phi=60^{\circ}$

(c) $\phi=30^{\circ}-$ Navier-Stokes solution: (d) $\phi=90^{\circ}$ (inviscid side walls) (e) $\phi=90^{\circ}$ (f) $\phi=60^{\circ}$ (g) $\phi=30^{\circ} \ldots \ldots \ldots$

5.20 Pressure contours with streamtraces at $\eta=0.0002 \%$ for NACA 64A007.5, $M_{\infty}=0.85, \alpha=0^{\circ}, R e_{c}=5.3 \times 10^{6}-$ Euler solution: (a) $\phi=90^{\circ}$ (b) $\phi=60^{\circ}$ (c) $\phi=30^{\circ}-$ Navier-Stokes solution: (d) $\phi=90^{\circ}$ (inviscid side walls) (e) $\phi=90^{\circ}$ (f) $\phi=60^{\circ}$ (g) $\phi=30^{\circ} \ldots \ldots$. . . . . . 95

5.21 Recirculation vortex at $\eta=0.0002 \%$ for NACA $64 \mathrm{~A} 007.5$ with $\phi=30^{\circ}$, $M_{\infty}=0.85, \alpha=0^{\circ}, R e_{c}=5.3 \times 10^{6}$ (Navier-Stokes solution) . . . . 98

5.22 Dissipation of the recirculation vortex for NACA 64A007.5 with $\phi=30^{\circ}$, $M_{\infty}=0.85, \alpha=0^{\circ}, R e_{c}=5.3 \times 10^{6}$ (Navier-Stokes solution) (a) $\eta=0.0002 \%$

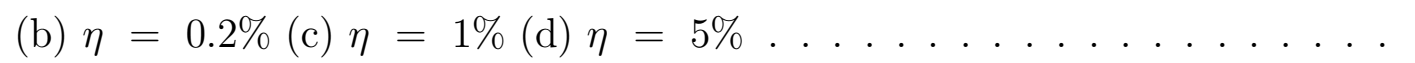

5.23 Pressure contours with streamtraces at $\eta=10 \%$ for NACA 64A007.5, $M_{\infty}=0.85, \alpha=0^{\circ}, R e_{c}=5.3 \times 10^{6}-$ Euler solution: (a) $\phi=90^{\circ}$ (b) $\phi=60^{\circ}$ (c) $\phi=30^{\circ}$ - Navier-Stokes solution: (d) $\phi=90^{\circ}$ (inviscid side walls) (e) $\phi=90^{\circ}$ (f) $\phi=60^{\circ}$ (g) $\phi=30^{\circ} \ldots \ldots$. . . . . . . 100

5.24 Pressure contours in a plane perpendicular to the strut planform at $\eta=50 \%$ for NACA 64A005, $M_{\infty}=0.85, \alpha=0^{\circ}, R e_{c}=5.3 \times 10^{6}$ - Euler solution: (a) $\phi=90^{\circ}$ (b) $\phi=60^{\circ}$ (c) $\phi=30^{\circ}$ - Navier-Stokes solution: (d) $\phi=90^{\circ}$ (inviscid side walls) (e) $\phi=90^{\circ}$ (f) $\phi=60^{\circ}$ (g) $\phi=30^{\circ} \ldots \ldots$. . . .

5.25 Pressure contours in a plane perpendicular to the strut planform at $\eta=50 \%$ for NACA 64A007.5, $M_{\infty}=0.85, \alpha=0^{\circ}, R e_{c}=5.3 \times 10^{6}-$ Euler solution: (a) $\phi=90^{\circ}$ (b) $\phi=60^{\circ}$ (c) $\phi=30^{\circ}$ - Navier-Stokes solution: (d) $\phi=90^{\circ}$ (inviscid side walls) (e) $\phi=90^{\circ}$ (f) $\phi=60^{\circ}$ (g) $\phi=30^{\circ} .103$

6.1 Methods to close the trailing edge (a) Original airfoil (b) Method 1: Extrapolation of the upper and lower surfaces (c) Method 2: Midpoint (d) Method 3: Removal of the last point on the lower surface (e) Method 4: Removal of the last point on the upper surface (f) Method 5: Displacement of the upper and lower surfaces using a linear variation . . . . . . . . . . . . . 106 
6.2 Pressure distribution with trailing edge closure for the NACA 64A007.5 airfoil, $M_{\infty}=0.85, \alpha=0^{\circ}$ (Euler solution) (a) Original airfoil (b) Method 1: Extrapolation of the upper and lower surfaces (c) Method 2: Midpoint (d) Method 3: Removal of the last point on the lower surface (e) Method 4: Removal of the last point on the upper surface (f) Method 5: Displacement of the upper and lower surfaces using a linear variation . . . . . . . . . . . . . . . . 108

6.3 Pressure distribution with trailing edge closure for the NACA 64A007.5 airfoil, $M_{\infty}=0.85, \alpha=0^{\circ}, R e_{c}=5.3 \times 10^{6}$ (Navier-Stokes solution) (a) Original airfoil (b) Method 1: Extrapolation of the upper and lower surfaces (c) Method 2: Midpoint (d) Method 3: Removal of the last point on the lower surface (e) Method 4: Removal of the last point on the upper surface (f) Method 5: Displacement of the upper and lower surfaces using a linear

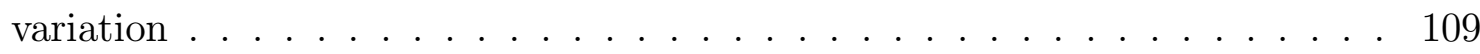

6.4 Forces acting on a 2-D airfoil . . . . . . . . . . . . . . . . . 112

6.5 Refinement of the inviscid grid at the leading edge of the NACA 64A007.5 airfoil (a) Fine grid with close-up view (b) Coarse grid with close-up view . . 114

6.6 Effect of the grid refinement at the leading edge of the NACA 64A007.5 airfoil, $M_{\infty}=0.85, \alpha=0^{\circ}$ (Euler solution) (a) Pressure coefficient as a function of the coordinate $x / c(\mathrm{~b})$ Pressure coefficient as a function of the coordinate $z / c 115$

6.7 Generation of a 2-D grid with VGRIDns . . . . . . . . . . . . . 116

6.8 Grid refinement at the leading edge of the NACA 64A005 airfoil (a) Fine grid (b) Coarse grid . . . . . . . . . . . . . . . . . . . 117

6.9 Effect of the grid refinement at the leading edge of the NACA 64A005 airfoil, $M_{\infty}=0.85, \alpha=0^{\circ}, R e_{c}=5.3 \times 10^{6}$ (Navier-Stokes solution) (a) Pressure distribution on the airfoil surface (b) Close-up view in the vicinity of the leading edge . . . . . . . . . . . . . . . . . . 118

6.10 Grid refinement at the trailing edge (a) Fine grid (b) Coarse grid . . . . . . 119 
6.11 Effect of the grid refinement at the trailing edge of the NACA 64A007.5 airfoil, $M_{\infty}=0.85, \alpha=0^{\circ}, R e_{c}=5.3 \times 10^{6}$ (Navier-Stokes solution) (a) Pressure distribution on the airfoil surface (b) Close-up view in the vicinity of the trailing edge . . . . . . . . . . . . . . . . . . . . 120

6.12 Grid crossing problem (a) Triangulation of a wing planform (b) Comparison of the original and the discretized geometries at the trailing edge . . . . . . .

6.13 A sliver next to a normal cell (a) Perspective view (b) View where the sliver has negligible thickness . . . . . . . . . . . . . . . . . . 122

6.14 Tetrahedral cell and the normal associated with each face . . . . . . . . . . 124

6.15 Location of the slivers in the grid for a strut inclined $60^{\circ}$ with respect to two flat walls (a) Inviscid grid (143 slivers): entire computational domain and close-up view of the strut (b) Viscous grid (5,678 slivers): entire computational domain and close-up view of the strut . . . . . . . . . . . 125

6.16 Distribution and cumulative distribution of the grid quality criteria for the inviscid grid (a) Sliver quality measure $Q_{1}$ (b) Skewness quality measure $Q_{2}$ (c) Maximum angle . . . . . . . . . . . . . . . . . . . . 126

6.17 Distribution and cumulative distribution of the grid quality criteria for the viscous grid (a) Sliver quality measure $Q_{1}$ (b) Skewness quality measure $Q_{2}$ (c) Maximum angle . . . . . . . . . . . . . . . . . . . . . . 127 


\section{List of Tables}

2.1 Aerodynamic interference in subsonic flow $\ldots \ldots \ldots \ldots$

2.2 Aerodynamic interference in transonic flow $\left(M_{\infty}=0.6-1.2\right) \ldots \ldots 11$

2.3 Aerodynamic interference in supersonic flow . . . . . . . . . . . . 15

4.1 Geometry of the strut-braced wing concept SS5 $\ldots \ldots \ldots$. . . . . . 48

4.2 Grid convergence study for the wing alone, $M_{\infty}=0.85, \alpha_{\text {root }}=2.36^{\circ}(\alpha=$

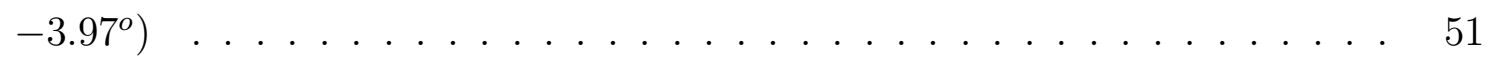

4.3 Grid characteristics for the various SS5 strut-braced wing configurations . . . 57

4.4 Lift and drag coefficients of the SS5 strut-braced wing configurations, $M_{\infty}=0.85$, $\alpha_{\text {root }}=2.36^{\circ}\left(\alpha=-3.97^{\circ}\right) \ldots \ldots \ldots \ldots \ldots \ldots \ldots \ldots \ldots \ldots \ldots \ldots \ldots$

5.1 Side wall boundary layer dimensions $1050 \mathrm{~mm}$ upstream of the strut . . . . 67

5.2 Normal force coefficient $C_{n}$ along the wing span $\ldots \ldots \ldots 67$

5.3 Grid characteristics . . . . . . . . . . . . . . . . . . . . 72

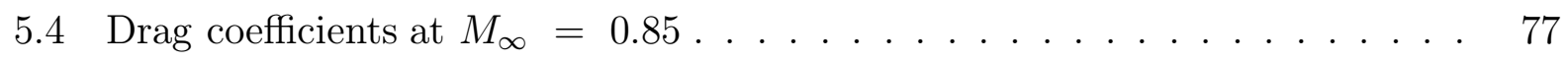

5.5 Grid convergence for the NACA 64A007.5 strut - $\phi=90^{\circ}, M_{\infty}=0.85$, $\alpha=0^{o}, R e_{c}=5.3 \times 10^{6} \ldots \ldots \ldots \ldots$

5.6 Interference drag coefficients . . . . . . . . . . . . . . . . . . . . . 89

6.1 Lift and drag coefficients for various trailing edge closure methods . . . . . . 110 
6.2 Lift and drag coefficients for various grid refinements at the leading edge of the NACA 64 A007.5 airfoil, $M_{\infty}=0.85, \alpha=0^{\circ} \ldots \ldots \ldots 114$

6.3 Lift and drag coefficients for various grid refinements at the leading edge of the NACA $64 \mathrm{~A} 005$ airfoil, $M_{\infty}=0.85, \alpha=0^{\circ}, R e_{c}=5.3 \times 10^{6} \ldots 117$

6.4 Lift and drag coefficients for various grid refinements at the trailing edge of the NACA 64A007.5 airfoil, $M_{\infty}=0.85, \alpha=0^{\circ}, R e_{c}=5.3 \times 10^{6} \ldots 119$ 


\title{
Nomenclature
}

\author{
speed of sound \\ $a_{n}, s, S \quad$ strength, spacing, and stretching of a grid source \\ $b \quad$ span \\ $c \quad$ chord \\ $C_{d} \quad$ drag coefficient of a 2-D airfoil \\ $\Delta C_{D} \quad$ increment of the drag coefficient \\ $C_{D} \quad$ drag coefficient of a 3-D body \\ $C_{D_{p}} \quad$ pressure drag coefficient \\ $C_{D_{v}} \quad$ skin friction drag coefficient \\ $C_{D_{S}} \quad$ drag coefficient of the strut alone \\ $C_{D_{S+W}} \quad$ drag coefficient of the strut-wall configuration \\ $C_{D_{\text {interf }}} \quad$ interference drag coefficient, Eq. 5.10 \\ $C_{D_{t o t}} \quad$ total drag coefficient \\ $C_{l} \quad$ lift coefficient of a 2-D airfoil \\ $C_{L} \quad$ lift coefficient of a $3-\mathrm{D}$ body \\ $C_{L_{S}} \quad$ lift coefficient of the strut alone \\ $C_{L_{S+W}} \quad$ lift coefficient of the strut-wall configuration \\ $C_{n} \quad$ normal force coefficient \\ $C_{p} \quad$ pressure coefficient \\ $C_{p, u}, C_{p, l}$ pressure coefficient on the upper and lower airfoil surface \\ CFL Courant-Friedrich-Levy number \\ $D_{\text {interf }} \quad$ interference drag, Eq. 5.9 \\ $D_{S} \quad$ drag of the strut alone \\ $D_{S+W} \quad$ drag of the strut-wall configuration \\ $E \quad$ total energy per unit volume \\ $\overrightarrow{\mathbf{F}}_{\mathbf{i}} \quad$ inviscid flux \\ $\mathbf{f}, \mathbf{g}, \mathbf{h} \quad$ Cartesian components of the inviscid flux \\ $\overrightarrow{\mathbf{F}}_{\mathbf{v}} \quad$ viscous flux \\ $\mathbf{f}_{\mathbf{v}}, \mathbf{g}_{\mathbf{v}}, \mathbf{h}_{\mathbf{v}}$ Cartesian components of the viscous flux \\ $M_{\infty} \quad$ freestream Mach number \\ $\hat{\mathbf{n}} \quad$ outward pointing normal \\ $\hat{n}_{x}, \hat{n}_{y}, \hat{n}_{z} \quad$ Cartesian components of the normal
}




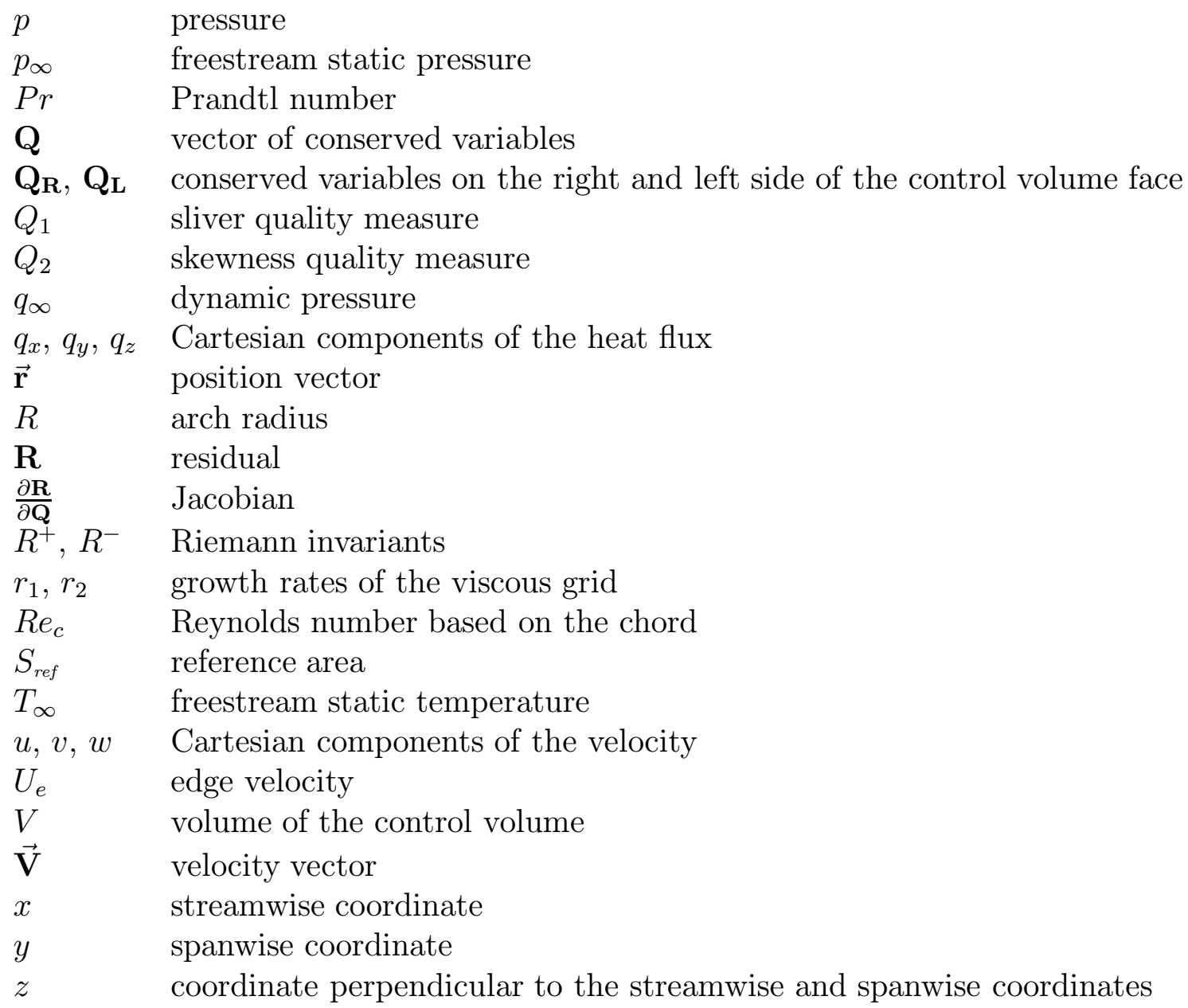




\section{Chapter 1}

\section{Introduction}

In 1996, Dennis Bushnell, chief scientist at the NASA Langley Research Center, challenged the Virginia Tech Multidisciplinary Analysis and Design (MAD) Center to apply Multidisciplinary Design Optimization (MDO) tools to the study of the strut-braced wing aircraft concept $[1,2,3]$. Within the MDO framework, the structural and aerodynamic analysis modules are linked together with an optimizer in order to minimize the aircraft take-off gross weight or any other suitable objective function subject to some constraints. The synergism that is obtained within this MDO context achieves a substantial reduction of the aircraft weight compared to an equivalent cantilever design with the same mission profile.

From the aerodynamics standpoint, the interference drag between the wing, the strut, and the fuselage is a major obstacle in designing a transonic strut-braced wing airplane. Subsequent sections of this work will deal with estimating the penalties associated with such junctions using Computational Fluid Dynamics (CFD) methodology on unstructured grids. The dissertation will be organized as follows:

- Chapter 2 presents a survey of the literature about innovative aircraft concepts and the phenomenon of aerodynamic interference in all speed regimes.

- Chapter 3 describes the CFD tools employed in this research. Details are given about the unstructured grid generation codes and the flow solver.

- Chapter 4 shows the application of the CFD methodology to a strut-braced wing design obtained with the MDO tools. The effect of employing a straight strut is compared with the benefits of employing an arch-shaped strut. 
- Chapter 5 discusses the application of a CFD approach to estimate the interference drag penalty of general strut-wall junctions. An expression for the interference drag is obtained as a function of the thickness of the strut, the Reynolds number of the flow, and the angle made by the strut with the nearby wall.

- Chapter 6 presents some of the challenges facing CFD today. Trailing edge closure, grid refinement, volume grid generation, and slivers are some of the topics dicussed in that chapter.

The dissertation ends with concluding remarks and recommendations for future work in the field. 


\section{Chapter 2}

\section{Literature Review}

The literature contains useful information about the aerodynamic interference and the emergence of novel designs for commercial transports. The most innovative airplane concepts about to challenge the position held by cantilever wing airplanes will be discussed first. Then, a discussion of the various studies made of the interference phenomenon will be presented for the subsonic, transonic, and supersonic speed regimes.

\subsection{Innovative Aircraft Concepts}

In today's aviation industry, cantilever wing designs are the most common configuration used for transonic civil transport aircraft. The Boeing 747-400 [4] is an example of that type of airplane. It has a wing sweep of 37.5 degrees and flies at 35,000 ft at a cruise Mach number of 0.85 . Over the years, this design has been scaled to allow the airplane to carry an increased payload without making major alterations to the concept itself.

In an attempt to seek a major improvement in the performance of transport airplanes, several innovative concepts have been studied in recent years. McMasters and Kroo [5] investigated the benefits of a 600-passenger concept with canard and a C-wing. They also looked at the design of a very large subsonic transport airplane configured as a flying boat. But the most promising concepts for the future of commercial transonic transport are the blended wing body, the inboard wing, the joined wing, and the strut-braced wing designs.

The blended wing body [6] consists in blending the passenger cabin, cargo structure, and 
propulsion system into the inboard wing. This results in a highly integrated design that exhibits large aerodynamics benefits compared to its cantilever counterpart.

The inboard wing concept [7] provides a way to increase the payload capacity without exceeding the dimensions of conventional airplane designs. The wing is located between two fuselages mounted at the wing tips. Because of the presence of bodies at the wing tips, it is suggested that this airplane eliminates or reduces the wing tip vortices found on conventional designs and hence, the induced drag.

The joined wing [8] aircraft employs a swept forward wing and a swept backward wing joined at the tip. The wings form a diamond shape from the top view and the front view. The root of the swept forward wing is fixed at the tail of the airplane. The most important benefits [9] of this configuration over the conventional airplanes are the reduced structural weight and the lower induced and overall drag. Preliminary design of joined wing aircraft led to the testing of several models in wind tunnel $[10,11]$. Recently, an MDO approach was applied to the concept to seek a better synergy among the various disciplines involved in the design of the aircraft. Gallman et al. [9] designed the aircraft for minimum Direct Operating Cost (DOC) and showed a $4 \%$ increase in DOC over a cantilever comparator. Structural optimization employing a minimum weight optimization approach or a fully stressed design method was also applied to the joined wing concept by Gallman and Kroo [12]. The addition of a buckling constraint produced a design that is more expensive to operate than its cantilever counterpart.

A derivative of the joined wing concept is the boxplane [8] or simply boxwing. The main difference with the joined wing is that the wings don't form a diamond shape in the front view. Instead, vertical sections join the tips of the forward and backward swept wings. Wolkovitch [8] mentions that this configuration achieves a significant reduction of the induced drag compared to a conventional wing.

An innovative concept is the strut-braced wing. The strut-braced wing configuration consists in adding a strut to a conventional airplane configuration to offer additional support for the wing. Pfenninger [13] proposed the concept as a means of reducing the induced drag through an increase of the wing aspect ratio. By employing a strut, the bending moment at the wing root is reduced, and it becomes possible to employ thinner airfoil sections for the main wing. This allows a reduction of the wing sweep, thus providing more laminar 
flow and hence less skin friction drag on the wing surface. Jobe et al. [14] presented the preliminary design of a large strut-braced wing airplane and compared it with the result of an optimization study performed on a cantilever wing design. They concluded that to achieve minimum fuel, it was necessary to have a wing of high aspect ratio, low sweep and small thickness to chord ratio. The wing deflection of the cantilever design during taxi was unacceptable but employment of a strut reduced that deflection to a reasonable level.

Grasmeyer [1], Gern et al. [2], and Gundlach et al. [3] presented the approach employed to study the benefits of the strut-braced wing concept within an MDO framework. The strutbraced wing was designed to carry 325 passengers at a cruise Mach number of 0.85 and to have a range of 7,500 nautical miles. The evaluation of the aerodynamic parameters was based on simple methods and the interference drag of the wing with the strut was determined from offline CFD analyses. For the structures, the determination of the bending material weight of the wing was based on a hexagonal wing box model that takes into account the torsional stiffness of the wing. The deformation of the wing under load conditions was considered. The strut was designed with a telescoping sleeve mechanism to prevent buckling in negative $g$ load conditions. Flutter analyses performed offline revealed that the airplane is safe to fly within the prescribed flight envelope. The resulting strut-braced wing design was shown to have a significantly lower take-off gross weight than a baseline cantilever design similar to a Boeing 777. It also employed less fuel and had smaller engines, both of which contribute to a reduction of the gas emissions and the noise level. Gundlach et al. [3] optimized the strutbraced wing and the cantilever designs for minimum take-off gross weight and for minimum fuel. The effect of changing the mission range to 4,000 nautical miles was investigated. All those analyses confirmed the benefits of the strut-braced wing compared to its cantilever counterpart.

\section{$2.2 \quad$ Interference Drag}

In this section, the phenomenon of aerodynamic interference is discussed in detail. The information is categorized according to the speed regime, whether it is in subsonic, transonic, or supersonic flow. Emphasis is put on wing-body, wing-pylon, and strut-endwall configurations. The measured or computed quantities are also summarized in tables. The following acronyms are employed in those tables: 
- B.L.: boundary layer

- RANS: Reynolds-averaged Navier-Stokes equations

- TLNS: thin-layer Navier-Stokes equations

- TSD: transonic small disturbances

- SLNS: slender-layer Navier-Stokes equations

- VLM: vortex-lattice method

\subsubsection{Subsonic Flow}

The work done by many researchers in the subsonic flow speed range is presented in Table 2.1.

Hoerner [24] performed experiments and collected much information related to interference drag of struts in subsonic flow. In some instances, he was able to correlate the interference drag with a simple equation to aid in estimating the drag of various configurations. The case of a strut perpendicular to a wall was studied for several strut thicknesses. The trends suggested that favorable interference drag is possible for thin struts. The interference drag increases significantly when the strut thickness is increased. By varying the strut sweep, it was observed that a substantial reduction in the interfernce drag can be achieved. When the strut is tilted laterally from its position perpendicular to the wall, flow separation in the junction produces a large increase in the interference drag coefficient.

Kubendran et al. [28] conducted an experiment for the flow around a wing-fuselage juncture. The juncture was simulated by attaching a body of constant thickness with an elliptical leading edge radius to a flat plate. The body was placed perpendicular to the flat plate. They obtained favorable interference equivalent to $-3 \%$ of the total drag of the plate and body in isolation. Barber [16] measured the intersection losses for a strut perpendicular to a wall in a low-speed wind tunnel. The struts studied had $t / c$ ratios of $20 \%$ and $30 \%$. Lower drag penalty was observed for thicker incident boundary layers than for thinner ones. Also, the dependence on the flow incidence angle was markedly smaller for a thicker boundary layer. In subsonic flow, Roach and Turner [38] tested a number of strut sections perpendicular to a wall. For streamlined strut sections of small aspect ratio, the secondary losses could be 
Table 2.1: Aerodynamic interference in subsonic flow

\begin{tabular}{|c|c|c|c|c|c|c|c|c|}
\hline \multirow[t]{2}{*}{ Ref. } & \multirow[t]{2}{*}{ Authors } & \multirow[t]{2}{*}{ Year } & \multirow[t]{2}{*}{ Configuration } & \multirow[t]{2}{*}{ Type of study } & \multirow{2}{*}{$\begin{array}{c}\text { If numerical, } \\
\text { flow equations solved }\end{array}$} & \multicolumn{3}{|c|}{ Data } \\
\hline & & & & & & Pressure & Forces & Others \\
\hline$[15]$ & $\begin{array}{l}\text { Ahmed and } \\
\text { Khan }\end{array}$ & 1995 & wing-body jct & Experimental & & $\mathrm{X}$ & & $\begin{array}{l}\text { velocity profiles, } \\
\text { oil viz. }\end{array}$ \\
\hline$[16]$ & Barber & 1978 & strut-endwall & Experimental & & $\bar{X}$ & $\bar{X}$ & oil viz. \\
\hline$[17]$ & Burke & 1989 & wing-body jct & Numerical & $\overline{\text { RANS }}$ & $\overline{\mathrm{X}}$ & & $\begin{array}{l}\text { velocity profiles, } \\
\text { streamtraces }\end{array}$ \\
\hline$[18]$ & $\begin{array}{l}\text { Chang and } \\
\text { Gessner }\end{array}$ & 1991 & wing-body jct & Experimental & & $\overline{\mathrm{X}}$ & & $\begin{array}{l}\text { velocity profiles } \\
\text { and contours, } \\
\text { turb. quantities }\end{array}$ \\
\hline$[19]$ & $\begin{array}{c}\text { Chen and } \\
\text { Hung }\end{array}$ & 1992 & cylinder-endwall & Numerical & RANS & $\mathrm{X}$ & & streamtraces \\
\hline$[20]$ & $\begin{array}{l}\text { Devenport } \\
\text { and Simpson }\end{array}$ & 1990 & wing-body jct & Experimental & & $\mathrm{X}$ & & $\begin{array}{l}\text { turb. quantities, } \\
\text { velocity, } \\
\text { oil viz. }\end{array}$ \\
\hline$[21]$ & $\begin{array}{l}\text { Devenport } \\
\text { et al. }\end{array}$ & 1990 & wing-body jct & Experimental & & $\mathrm{X}$ & & $\begin{array}{l}\text { turb. quantities, } \\
\text { oil viz. }\end{array}$ \\
\hline$[22]$ & $\begin{array}{l}\text { Devenport } \\
\quad \text { et al. }\end{array}$ & 1992 & wing-body jct & Experimental & & $\mathrm{X}$ & & $\begin{array}{l}\text { turb. quantities, } \\
\text { velocity, } \\
\text { oil viz. }\end{array}$ \\
\hline$[23]$ & Fleming et al. & 1992 & wing-body jct & Experimental & & & & $\begin{array}{l}\text { velocity profiles, } \\
\text { turb. quantities }\end{array}$ \\
\hline$[24]$ & Hoerner & 1965 & strut-endwall & Experimental & & & $\mathrm{X}$ & \\
\hline 25$]$ & Kaykayoglu & 1996 & $\begin{array}{l}\text { wing-fuselage-- } \\
\text { pylon-store }\end{array}$ & Numerical & unsteady VLM & & $\bar{X}$ & \\
\hline$[26]$ & $\begin{array}{l}\text { Khan and } \\
\text { Ahmed }\end{array}$ & 1996 & wing-body jct & Experimental & & & & turb. quantities \\
\hline$[27]$ & $\begin{array}{l}\text { Krautheim } \\
\text { et al. }\end{array}$ & 1997 & strut-walls & Experimental & & $\bar{X}$ & & velocity \\
\hline$[28]$ & Kubendran et al. & 1984 & wing-body jct & Experimental & & $\overline{\mathrm{X}}$ & $\overline{\mathrm{X}}$ & velocity profiles, \\
\hline$[29]$ & $\begin{array}{c}\text { Kubendran and } \\
\text { Harvey }\end{array}$ & 1985 & wing-body jct & Experimental & & $\bar{X}$ & & velocity profiles \\
\hline$[30]$ & Kubendran et al. & 1986 & wing-body jct & Experimental & & & & $\begin{array}{l}\text { velocity profiles, } \\
\text { turb. quantities }\end{array}$ \\
\hline [31] & Maughmer & 1987 & wing-fuselage & Experimental & & $\bar{X}$ & $\bar{X}$ & \\
\hline$[32]$ & $\begin{array}{l}\text { Ölçmen and } \\
\text { Simpson }\end{array}$ & 1994 & wing-body jct & Experimental & & $\mathrm{X}$ & & $\begin{array}{l}\text { velocity profiles, } \\
\text { oil viz. }\end{array}$ \\
\hline$[33]$ & $\begin{array}{l}\text { Ölçmen and } \\
\text { Simpson }\end{array}$ & 1996 & wing-body jct & Experimental & & $\mathrm{X}$ & & $\begin{array}{l}\text { velocity profiles, } \\
\text { turb. quantities }\end{array}$ \\
\hline$[34]$ & $\begin{array}{l}\text { Ölçmen and } \\
\text { Simpson }\end{array}$ & 1997 & wing-body jct & Experimental & & & & $\begin{array}{l}\text { velocity profiles, } \\
\text { turb. quantities, } \\
\text { streamlines }\end{array}$ \\
\hline$[35]$ & $\begin{array}{l}\text { Özcan and } \\
\text { Ölçmen }\end{array}$ & 1988 & wing-body jct & Experimental & & $\mathrm{X}$ & $\mathrm{X}$ & $\begin{array}{c}\text { streamtraces } \\
\text { velocity profiles }\end{array}$ \\
\hline$[36]$ & $\begin{array}{l}\text { Paul and } \\
\text { Carlson }\end{array}$ & 1992 & wing-body jct & Numerical & $\begin{array}{c}\text { incompressible } \\
\text { with TLNS and SLNS } \\
\text { assumptions }\end{array}$ & $\bar{X}$ & & $\begin{array}{l}\text { velocity profiles, } \\
\text { streamtraces }\end{array}$ \\
\hline$[37]$ & $\begin{array}{l}\text { Pierce and } \\
\text { Nath } \\
\end{array}$ & 1990 & strut-endwall & Experimental & & $\overline{\mathrm{X}}$ & $\overline{\mathrm{X}}$ & velocity contours \\
\hline$[38]$ & $\begin{array}{l}\text { Roach and } \\
\text { Turner }\end{array}$ & 1985 & strut-endwall & Experimental & & & $\bar{X}$ & pressure contours \\
\hline$[39]$ & $\begin{array}{l}\text { Shabaka and } \\
\text { Bradshaw }\end{array}$ & 1981 & wing-body jct & Experimental & & & & $\begin{array}{l}\text { velocity contours } \\
\text { and vectors, } \\
\text { vorticity }\end{array}$ \\
\hline$[40]$ & Thwaites & 1960 & $\begin{array}{l}\text { wing-wing and } \\
\text { wing-body jcts }\end{array}$ & Theoretical & incompressible & & $\bar{X}$ & $\begin{array}{c}\text { velocity } \\
\text { distribution } \\
\end{array}$ \\
\hline
\end{tabular}


reduced to a correlation in terms of the Reynolds number of the flow, the Mach number, the thickness-to-chord ratio of the strut and the displacement thickness of the incident boundary layer. Pierce and Nath [37] investigated the interference drag of a streamlined body with a thickness-to-chord ratio of $42.6 \%$. The study showed a significant drag increase due to the junction. When compared to similar studies, the results confirmed the dependence of the interference drag on the thickness-to-chord ratio of the strut.

Ref. [40] presents some theoretical developments to estimate the variation of the chordwise velocity distribution for various junctions in subsonic flow. For the case of perpendicular rectangular wings, the expressions derived provide a crude estimate of the behavior of the flow velocity. The flow velocity on the wing surface is in some cases considerably higher near the junction than some distance away from it. Therefore, thinning the airfoil sections near the junction is seen as a way to reduce the onset of compressibility effects and the tendency of the boundary layer to separate in that region. Similar analyses are presented for two intersecting swept wings and several wing-body junctions.

The subsonic flow about a wing-body junction is a topic that is studied extensively in the literature. It serves an important purpose in the determination of the turbulent quantities of the flow. Several experiments and numerical calculations have been conducted on the topic. Ref. [41] provides a very good survey of the experiments done on wing-body junctions in subsonic flow.

Shabaka and Bradshaw [39] measured the turbulent flow and the decay of the horseshoe vortex forming around an idealized wing-body junction. Devenport and Simpson [20] measured the turbulent quantities upstream of a wing-body juncture to obtain some insight in the time-dependent and the time-averaged structure of the flow in that region. They also observed the separation that occurs because of the adverse pressure gradient produced by the wing. Abdulla-Altaii and Raj [41] identified a dominant horseshoe vortex and a weaker corner vortex in the wake of a wing-body junction. Ölçmen and Simpson [33] focused on the vicinity of a 3-D separation line and in the region where the horseshoe vortex forms around the wing. In addition, they investigated the flow in the vicinity of the wing-body junction [34]. Özcan and Ölçmen [35] measured the flow behind a wing-body junction at high angle of attack. Ölçmen and Simpson [32] measured the pressure fluctuations in the flow around several wing-body junctions and showed that they are dependent upon the wing 
geometry. Chang and Gessner [18] and Fleming et al. [23] measured the characteristics of the turbulent flow in the vicinity and downstream of the wing-body junction. For the analysis of the subsonic flow in wing-body junctions, Navier-Stokes flow solvers have been applied successfully by Burke [17] and Paul and Carlson [36].

For wing-body junctions, Ahmed and Khan [15] reported that the location of the juncture vortex is very sensitive to the sweep. The vortex moves towards the wing for back sweep and away for forward sweep. Khan and Ahmed [26] determined that the effect of the wing sweep on the flow structure is significant as the forward swept wing has a lower turbulence kinetic energy level. Kubendran et al. [30] found that the slenderness ratio of the wing leading edge affects the location and the strength of the vortex that wraps around the wing.

The effect of employing fillets at the leading edge of wing-body junctures was assessed experimentally by Kubendran and Harvey [29] and Devenport et al. [21, 22]. Kubendran and Harvey [29] noted that the fillets provide a reduction in the measured drag coefficient of the arrangement at moderate angle of attack. However, past a certain point in fillet size, the flow characteristics deteriorate significantly. Devenport et al. [21] measured the turbulent flow around an idealized wing-body junction. A fillet of uniform radius was wrapped around the base of the wing. The fillet had the detrimental effect of increasing the strength of the horseshoe vortex. In addition, the effective nose radius of the wing was increased. The angle of attack and the thickness of the incoming boundary layer did not change the qualitative behavior of the vortex in the presence of the fillet. Another experimental study conducted by Devenport et al. [22] was more successful in designing a leading edge fillet consisting of a fairing between the nose of the wing and the body surface upstream. At zero angle of attack, the leading edge separation and the horseshoe vortex were eliminated. At nonzero angle of attack, the fillet had a desirable effect on the behavior of the flow. Maughmer [31] reported that an extension of the leading edge of the wing where it intersects the fuselage reduces the strength of the vortex in the vicinity of the junction and thus decreases the drag significantly.

Slooff [42] reports some of the issues related to inverse design methods where a target pressure distribution has to be matched. For those methods, three-dimensional problems are ill-posed because small differences in the target pressure distribution lead to large differences in the final geometry. This is illustrated by the example of the design of a wing-body junction. 
In the vicinity of the junction, a slight difference in the target pressure distribution produces wing sections and twist distributions that are totally different from each other.

Kaykayoglu [25] applied an unsteady vortex lattice method to calculate the loads on a wing-fuselage-pylon-store configuration in incompressible flow. The position of the pylon and the store under the wing was shown to have some influence on the aerodynamic coefficients. Overall, the method proved to be able to simulate the interference phenomenon that takes place between the wing-fuselage-pylon and the store when it is released.

\subsubsection{Transonic Flow}

In transonic flow, numerous studies have been performed on multibody, wing-pylon and wing-body configurations, with more emphasis on numerical calculations. This can be seen in Table 2.2.

Bartelheimer et al. [46] performed an experiment on a wing placed perpendicular to the walls of a wind tunnel. Pressure measurements were made along the span of the strut. The boundary layer characteristics were also determined along the walls to help reproduce the test conditions with numerical tools. The data obtained was suitable only for "in-tunnel" CFD validation, because the strut was enclosed by solid wind tunnel walls above, below and on the sides. It will be used in Chapter 5 for validation purposes.

For wind-tunnel testing in the transonic speed range, Suhs [78] proposed a computational method to estimate the interference produced by model support struts. The method involved the solution of the Euler equations over a complete model with and without the supporting strut. A measure of the interference was obtained by calculating the difference in pressure coefficients between the two arrangements.

Cottrell et al. $[52,53]$ measured the interference effect on a multibody configuration at Mach numbers ranging from 0.6 to 1.2. Configurations containing one, two, and three finned [52] and unfinned [53] stores were tested in a wind tunnel for various angles of attack. The experimental data indicated that the interference effects are greatest near unit Mach number and get smaller when the freestream conditions are increased or decreased from sonic conditions. Chapman et al. [50] studied similar arrangements of unfinned stores for the same speed range. For all cases, the normal interference force tends to pull the bodies 
Table 2.2: Aerodynamic interference in transonic flow $\left(M_{\infty}=0.6-1.2\right)$

\begin{tabular}{|c|c|c|c|c|c|c|c|c|}
\hline \multirow[t]{2}{*}{ Ref. } & \multirow[t]{2}{*}{ Authors } & \multirow[t]{2}{*}{ Year } & \multirow[t]{2}{*}{ Configuration } & \multirow[t]{2}{*}{ Type of study } & \multirow{2}{*}{$\begin{array}{c}\text { If numerical, } \\
\text { flow equations solved }\end{array}$} & \multicolumn{3}{|c|}{ Data } \\
\hline & & & & & & Pressure & Forces & Others \\
\hline$[43]$ & Agrawal et al. & 1991 & wing-fuselage & Numerical & Euler & $\bar{X}$ & $\mathrm{X}$ & \\
\hline$[44]$ & $\begin{array}{l}\text { Arabshahi and } \\
\text { Whitfield }\end{array}$ & 1989 & wing-pylon-store & Numerical & Euler & $\mathrm{X}$ & & \\
\hline$[45]$ & Baxendale & 1990 & wing-body-pylon-store & Numerical & Euler & $\bar{X}$ & & \\
\hline$[46]$ & Bartelheimer et al. & 1994 & wing-endwalls & Experimental & & $\mathrm{X}$ & & side wall B.L. \\
\hline$[47]$ & Benek et al. & 1987 & multiple bodies & Numerical & TLNS & $\mathrm{X}$ & & streamtraces \\
\hline \multirow[t]{2}{*}[48]{} & \multirow{2}{*}{$\begin{array}{l}\text { Carlson and } \\
\text { Lamb }\end{array}$} & \multirow[t]{2}{*}{1987} & \multirow[t]{2}{*}{ wing-body-pylon } & Experimental & & $\mathrm{X}$ & $\mathrm{X}$ & \\
\hline & & & & Numerical & $\begin{array}{l}\text { incompressible } \\
\text { panel method }\end{array}$ & & $\mathrm{X}$ & \\
\hline$[49]$ & Chandrasekaran & 1988 & wing-body-pylon & Numerical & $\begin{array}{c}\text { Euler with } \\
\text { interactive B.L. }\end{array}$ & $\mathrm{X}$ & $\bar{X}$ & \\
\hline \multirow{2}{*}[50]{} & \multirow{2}{*}{ Chapman et al. } & \multirow{2}{*}{1992} & \multirow{2}{*}{ multiple stores } & Experimental & & & $\mathrm{X}$ & oil visualization \\
\hline & & & & Numerical & TLNS & $\bar{X}$ & $\mathrm{X}$ & \\
\hline$[51]$ & Chesser et al. & 1995 & aircraft-store & Numerical & Euler & $\bar{X}$ & $\bar{X}$ & \\
\hline \multirow[t]{2}{*}[52]{} & \multirow{2}{*}{$\begin{array}{l}\text { Cottrell and } \\
\text { Lijewski }\end{array}$} & \multirow[t]{2}{*}{1988} & \multirow[t]{2}{*}{ multiple stores } & Experimental & & $\bar{X}$ & & oil visualization \\
\hline & & & & Numerical & Euler & $\mathrm{X}$ & & \\
\hline \multirow[t]{2}{*}[53]{} & \multirow{2}{*}{ Cottrell et al. } & \multirow{2}{*}{1988} & \multirow[t]{2}{*}{ multiple stores } & Experimental & & $\mathrm{X}$ & & oil visualization \\
\hline & & & & Numerical & Euler & $\mathrm{X}$ & & \\
\hline$[54]$ & Deese et al. & 1984 & wing-fuselage & Numerical & Euler & $\bar{X}$ & & \\
\hline$[55]$ & $\begin{array}{c}\text { Deese and } \\
\text { Agarwal }\end{array}$ & 1988 & wing-body & Numerical & TLNS, SLNS & $\mathrm{X}$ & & \\
\hline$[56]$ & Ecer et al. & 1989 & wing-pylon-nacelle & Numerical & Euler & $\bar{X}$ & & \\
\hline$[57]$ & Fox et al. & 1991 & aircraft-stores & Numerical & Euler & $\mathrm{X}$ & & \\
\hline$[58]$ & $\begin{array}{l}\text { Fujii and } \\
\text { Obayashi }\end{array}$ & 1987 & wing-fuselage & Numerical & TLNS & $\bar{X}$ & & $\begin{array}{c}\text { streamtraces, } \\
\text { contours }\end{array}$ \\
\hline$[59]$ & Gea et al. & 1993 & wing-pylon & Numerical & TLNS & $\mathrm{X}$ & & $\begin{array}{c}\text { streamtraces, } \\
\text { contours }\end{array}$ \\
\hline$[60]$ & Jordan & 1992 & wing-pylon-store & Numerical & Euler & $\bar{X}$ & $\bar{X}$ & \\
\hline [61] & Kao et al. & 1993 & wing-body & Numerical & TLNS & $\mathrm{X}$ & & velocity vectors \\
\hline [62] & Lijewski & 1990 & multiple stores & Numerical & Euler & $\mathrm{X}$ & $\mathrm{X}$ & \\
\hline [63] & Lijewski & 1991 & wing-pylon-store & Numerical & Euler & $\mathrm{X}$ & $\mathrm{X}$ & \\
\hline$[64]$ & Lijewski & 1993 & wing-pylon-store & Numerical & Euler & $\bar{X}$ & $\mathrm{X}$ & \\
\hline [65] & $\begin{array}{c}\text { Lijewski and } \\
\text { Suhs }\end{array}$ & 1994 & wing-pylon-store & Numerical & Euler & $\mathrm{X}$ & $\bar{X}$ & \\
\hline$[66]$ & Lord and Zysman & 1986 & wing-pylon-nacelle & Numerical & $\begin{array}{c}\text { incompressible } \\
\text { panel method with } \\
\text { compressibility } \\
\text { correction }\end{array}$ & $\mathrm{X}$ & & \\
\hline$[67]$ & $\begin{array}{c}\text { Lynch and } \\
\text { Rizk }\end{array}$ & 1991 & multiple stores & Numerical & TLNS & $\mathrm{X}$ & $\mathrm{X}$ & \\
\hline$[68]$ & Madson et al. & 1994 & wing-pylon-store & Numerical & full-potential & $\bar{X}$ & $\mathrm{X}$ & \\
\hline$[69]$ & Meakin & 1992 & wing-pylon-store & Numerical & TLNS & $\mathrm{X}$ & $\mathrm{X}$ & \\
\hline$[70]$ & Naik & 1989 & wing-pylon & Numerical & Euler & $\mathrm{X}$ & $\mathrm{X}$ & \\
\hline$[71]$ & Naik et al. & 1993 & wing-body-pylon & Experimental & & $\mathrm{X}$ & $\mathrm{X}$ & oil visualization \\
\hline$[72]$ & $\begin{array}{c}\text { Newman and } \\
\text { Baysal }\end{array}$ & 1992 & wing-pylon-store & Numerical & Euler & $\mathrm{X}$ & & \\
\hline$[73]$ & Parikh et al. & 1992 & wing-pylon-store & Numerical & Euler & $\bar{X}$ & $\mathrm{X}$ & pressure contours \\
\hline$[74]$ & Potsdam et al. & 1993 & wing-pylon & Numerical & Euler & $\mathrm{X}$ & & streamtraces \\
\hline$[75]$ & Rosen & 1988 & wing-pylon-store & Numerical & TSD & $\overline{\mathrm{X}}$ & & \\
\hline$[76]$ & $\begin{array}{l}\text { Shankar and } \\
\text { Malmuth }\end{array}$ & 1980 & wing-body-pylon-store & Numerical & $\mathrm{TSD}$ & $\mathrm{X}$ & $\mathrm{X}$ & \\
\hline$[77]$ & Sundaram et al. & 1983 & wing-body-pylon-store & Numerical & panel method & $\bar{X}$ & & \\
\hline$[78]$ & Suhs & 1985 & wing-body-strut & Numerical & Euler & $\mathrm{X}$ & & \\
\hline$[79]$ & Tu et al. & 1992 & wing-pylon-store & Numerical & Euler & $\mathrm{X}$ & & \\
\hline$[80]$ & $\begin{array}{l}\text { Vatsa and } \\
\text { Wedan }\end{array}$ & 1988 & wing-endwall & Numerical & TLNS & $\mathrm{X}$ & & streamtraces \\
\hline
\end{tabular}


together, while the axial component increases the drag per body as more stores are added to the arrangement.

Cottrell et al. [52, 53] and Lijewski [62] obtained good results with Euler flow solvers to study single and multibody interference phenomenon as long as viscous effects were not important. For a three-body case, Lijewski [62] noted that the forces and moments are influenced mainly by the Mach number. Thin-layer Navier-Stokes flow solutions yielded valuable lessons as well. Benek et al. [47] successfully applied a thin-layer Navier-Stokes flow solver with grid overlapping to the study of the flow over a single body and a three-body arrangements. For multibody configurations, Chapman et al. [50] accurately predicted the trends in the experimental results, but not their value. Lynch and Rizk [67] observed that the interference level increases when the freestream Mach number is increased or when the distance between the bodies is reduced. Attractive forces were generated between the bodies of the configurations studied.

Although Euler and Navier-Stokes flow solvers are commonly applied to the study of wingpylon-store arrangements, approaches based on a velocity potential have been employed. In the early 1980's, Transonic Small Disturbance (TSD) theory was applied to compute the flowfield around a wing-fuselage-pylon-store combination by Shankar and Malmuth [76]. After the validation with experimental data showed good agreement with the calculations, the approach was extended to evaluate the effect of the pylon height and location, store size, angle of attack, and Mach number on the aerodynamic characteristics of the configuration. Sundaram et al. [77] presented computations made on a wing-body-pylon-store arrangement with TSD theory and a correction for viscous/inviscid interaction. The approach employed was satisfactory in predicting the flow characteristics for attached inviscid flows but was poor in regions of strong viscous/inviscid interaction. Rosen [75] employed a TSD approach to predict the flow around many configurations, including a wing-pylon-store case. Unsucessful attempts were made at extending the application of the subsonic panel code VSAERO to the calculation of transonic flow around a wing-pylon-nacelle configuration by Lord and Zysman [66] and over several wing-pylon combinations by Carlson and Lamb [48]. Recently, Madson et al. [68] employed a transonic full-potential approach to compute the flowfield around a wing-pylon-finned store geometry at Mach numbers of 0.95 and 1.20. The results were compared to wind tunnel measurements and to calculations using Euler and thin-layer Navier-Stokes approaches. The agreement of the forces and pressure distribution on the 
store with the experimental data was as good as for the Euler and Navier-Stokes solvers.

Wind tunnel tests and numerical calculations have been used extensively to try to improve the flow over pylons. Carlson and Lamb [48] tested various pylon sections under a wing in a transonic wind tunnel facility. The objective of the study was to determine the installation drag produced by the pylons. The toe-in angle of the pylons and the wing incidence were varied. Conventional pylons showed a region of strong interference inboard of the pylon accompanied by separation in some instances. The new concept of a "compression" pylon [48] was tested. It consists of a pylon with flat sides that continuously diverge for the section under the wing. This reduces the flow acceleration in the wing-pylon junction. Aft of the trailing edge, the pylon shape is closed. That pylon shape gave the lowest installed drag for both Mach numbers tested. Chandrasekaran [49] employed a three-dimensional Euler flow solver coupled with a boundary-layer interaction method to look at the transonic flow over wing-pylon arrangements. That approach was shown to be able to handle complex pylon shapes. It also confirmed the advantages of the compression pylons over their conventional counterparts.

Naik [70] employed a three-dimensional Euler flow solver to study the pylon geometries tested by Carlson and Lamb [48]. In addition, the potential of "hybrid" pylons was estimated. The hybrid pylon combines the advantages of a conventional pylon section with some aspects of the compression pylons to further reduce the interference drag. The numerical results highlighted the benefits of the compression pylons and the promises of hybrid pylon sections compared to conventional pylon shapes. Naik et al. [71] tested conventional, compression, and hybrid pylon sections in a transonic wind tunnel. Measured data indicated that the compression and hybrid pylons produce pressure distributions on the wing that are equivalent to the clean wing case. The separation regions were also small. Chesser et al. [51] compared the forces and moments generated on a store fixed to a F-15E airplane with two different pylon shapes. Euler analyses on embedded grids revealed that there was no significant benefit of the modified pylon shape compared to the original one. This assessment was confirmed later by wind tunnel tests.

The analysis and attempted geometry refinement of a wing-pylon junction was performed with two Euler flow solvers on unstructured grids by Potsdam et al. [74]. The poor features of the existing fillet in the junction of the pylon with the wing were highlighted. One of the 
CFD codes was then coupled with an inverse design method to provide some guidelines on how to improve the existing pylon section. The flow over the final pylon design was measured during flight test and validated with a Navier-Stokes flow solver. Gea et al. [59] improved an existing wing-pylon configuration by employing a Chimera-based Navier-Stokes flow solver.

Multiblock Euler flow solvers were used for flow prediction over a wing-pylon-nacelle combination by Ecer et al. [56], a wing-pylon-store arrangement by Arabshahi and Whitfield [44], and a wing-body-pylon-store configuration by Baxendale [45]. In the latter case, it was noted that the methodology was able to predict the flow in regions of high interference such as in the vicinity of the wing-pylon junctions, on the pylons and on the store. Lijewski [63, 64] and Lijewski and Suhs [65] employed a blocked and an overlapping-grid approaches with an Euler flow solver to calculate the pressure distribution and investigate the interference on a wing-pylon-store configuration in transonic flow.

Other approaches have been applied to the solution of the Euler equations. Newman and Baysal [72] employed a hybrid domain decomposition for the transonic flow over a wingpylon-finned store, Tu et al. [79] used unstructured grids to study the flow over a wing-pylonstore configuration in the transonic and supersonic speed range, and Fox et al. [57] analyzed the flow around an F-15E aircraft with finned stores with a grid embedding technique. All methods agreed well with experimental data. Parikh et al. [73] studied the transonic flow past a wing-pylon-finned store configuration using an unstructured Euler flow solver as well. The predicted pressure, forces and moments were in good agreement with the experimental data. The same configuration was analyzed successfully by Jordan [60] and Meakin [69] with a thin-layer Navier-Stokes solver.

Agrawal et al. [43] and Deese et al. [54] ran Euler flow solvers over wing-fuselage arrangements in transonic flow. Agrawal et al. [43] compared the accuracy of implicit finite-volume upwind, explicit finite-volume central-difference, and nonconservative upwind solvers. The methods predicted similar results except in regions where shock waves were expected and where the grid was too coarse. Overall, the implicit fine-volume upwind scheme provided the best results compared to experimental data for various mesh sizes.

Wing-fuselage combinations were studied with thin-layer Navier-Stokes flow solvers by Fujii and Obayashi [58], Vatsa and Wedan [80], and with thin-layer and slender-layer NavierStokes formulations by Deese and Agarwal [55]. Flow solutions presented by Fujii and 
Table 2.3: Aerodynamic interference in supersonic flow

\begin{tabular}{|c|c|c|c|c|c|c|c|c|}
\hline \multirow[t]{2}{*}{ Ref. } & \multirow[t]{2}{*}{ Authors } & \multirow[t]{2}{*}{ Year } & \multirow[t]{2}{*}{ Configuration } & \multirow[t]{2}{*}{ Type of study } & \multirow{2}{*}{$\begin{array}{c}\text { If numerical, } \\
\text { flow equations solved }\end{array}$} & \multicolumn{3}{|c|}{ Data } \\
\hline & & & & & & Pressure & Forces & Others \\
\hline$[81]$ & $\begin{array}{c}\text { Chen and } \\
\text { Liu }\end{array}$ & 1990 & wing-body-stores & Numerical & panel method & $\mathrm{X}$ & $\mathrm{X}$ & \\
\hline$[19]$ & $\begin{array}{l}\text { Chen and } \\
\text { Hung }\end{array}$ & 1992 & cylinder-endwall & Numerical & RANS & & & streamtraces \\
\hline$[82]$ & $\begin{array}{l}\text { Hung and } \\
\text { Buning }\end{array}$ & 1984 & fin-endwall & Numerical & TLNS & $\mathrm{X}$ & & $\begin{array}{c}\text { streamtraces, } \\
\text { contours }\end{array}$ \\
\hline$[83]$ & Knight et al. & 1992 & fin-endwall & Numerical & $\begin{array}{l}\text { 3-D RANS and } \\
\text { conical RANS }\end{array}$ & $\mathrm{X}$ & & $\begin{array}{l}\text { streamlines, } \\
\text { contours }\end{array}$ \\
\hline$[84]$ & Kulfan & 1990 & $\begin{array}{l}\text { supersonic } \\
\text { airplane }\end{array}$ & Theoretical & & & $\mathrm{X}$ & $\begin{array}{c}\text { conceptual } \\
\text { analysis }\end{array}$ \\
\hline$[85]$ & $\begin{array}{c}\text { Lakshmanan and } \\
\text { Tiwari }\end{array}$ & 1993 & wing-body jct & Numerical & RANS & $\mathrm{X}$ & & $\begin{array}{l}\text { streamlines, } \\
\text { contours }\end{array}$ \\
\hline$[86]$ & $\begin{array}{c}\text { Lakshmanan and } \\
\text { Tiwari }\end{array}$ & 1994 & wing-body jct & Numerical & TLNS & $\mathrm{X}$ & & $\begin{array}{c}\text { velocity profiles, } \\
\text { streamtraces }\end{array}$ \\
\hline$[87]$ & $\begin{array}{l}\text { McMaster and } \\
\text { Shang }\end{array}$ & 1988 & sweptback fin-wall & Numerical & mass-averaged N-S & & & streamtraces \\
\hline$[88]$ & $\begin{array}{c}\text { Philpott and } \\
\text { Zhao }\end{array}$ & 1984 & two cylinders & Experimental & & $\mathrm{X}$ & & $\begin{array}{c}\text { oil visualization, } \\
\text { schlieren }\end{array}$ \\
\hline [89] & Pritulo et al. & 1995 & wing-body & Numerical & Euler & $\mathrm{X}$ & $\mathrm{X}$ & \\
\hline \multirow[t]{2}{*}[90]{} & \multirow[t]{2}{*}{ Williams et al. } & \multirow[t]{2}{*}{1994} & \multirow[t]{2}{*}{ strut-endwall } & Experimental & & $\mathrm{X}$ & $\mathrm{X}$ & $\begin{array}{c}\text { vel. profiles, } \\
\text { B.L. characteristics }\end{array}$ \\
\hline & & & & Numerical & RANS & $\mathrm{X}$ & $\mathrm{X}$ & $\begin{array}{c}\text { vel. profiles, } \\
\text { B.L. characteristics }\end{array}$ \\
\hline [91] & Williams et al. & 1995 & strut-endwall & Numerical & RANS & $\mathrm{X}$ & & streamtraces \\
\hline \multirow[t]{2}{*}[92]{} & \multirow[t]{2}{*}{ Williams et al. } & \multirow[t]{2}{*}{1995} & \multirow[t]{2}{*}{ strut-endwall } & Experimental & & $\mathrm{X}$ & & streamlines \\
\hline & & & & Numerical & RANS & $\mathrm{X}$ & & streamtraces \\
\hline [93] & Williams et al. & 1995 & strut-endwall & Numerical & RANS & $\mathrm{X}$ & & streamlines \\
\hline \multirow[t]{2}{*}[94]{} & \multirow[t]{2}{*}{$\begin{array}{l}\text { Zemsch and } \\
\text { Degrez }\end{array}$} & \multirow[t]{2}{*}{1993} & \multirow[t]{2}{*}{ fin-flat plate } & Experimental & & $\mathrm{X}$ & & $\begin{array}{l}\text { oil visualization } \\
\text { velocity profiles }\end{array}$ \\
\hline & & & & Numerical & Euler & $\mathrm{X}$ & & streamtraces \\
\hline
\end{tabular}

Obayashi [58] showed the significant influence of the fuselage on the flowfield, especially in the vicinity of the wing root at high angle of attack. The impact of the junction flow was assessed also by Vatsa and Wedan [80] who studied the case of a swept NACA 0012 wing mounted to the side wall of a wind tunnel. The comparison of the numerical results with experimental data and with "free-air" computations showed a substantial influence of the side wall boundary layer on the flow over the wing. Kao et al. [61] validated a multiblock Navier-Stokes solver with experimental data for the transonic flow past a Boeing 747-200 airplane.

\subsubsection{Supersonic Flow}

A summary of the studies in the literature on the topic of aerodynamic interference in supersonic flow is presented in Table 2.3.

Kulfan [84] looked at ways to achieve favorable aerodynamic interference for a highspeed civil transport aircraft. The concepts of supersonic biplanes, wave riders, flat-top wing bodies and parasol wing were investigated. The parasol wing configuration showed the greatest promises in terms of lift-to-drag ratio improvements compared to a reference flat 
wing arrangement. The integration of the nacelle for low drag on a supersonic aircraft was shown to be similar to the parasol wing idea.

Williams et al. [92, 91, 90, 93] performed several numerical and experimental studies about the interference produced by struts in an annular duct in supersonic flow. Those analyses serve as applications to engine development. In Ref. [92], the configuration contained four struts located circumferentially in an annular duct. Measurements were compared with CFD calculations performed on structured grids with a Reynolds-averaged Navier-Stokes flow solver. Secondary flows were examined including the horseshoe vortices forming at the leading edge and trailing edge of the strut and at the junction of the strut with the endwall. Ref. [91] presented a discussion of the effect of varying the strut thickness on the flow. A Navier-Stokes solution scheme was employed to look at the horseshoe and corner vortices as well as boundary layer separation. Ref. [90] reported the numerical and experimental investigations of the turbulent boundary layer that develops along the side walls of an annular duct. Excellent agreement was achieved between the experimental data and the computations. Finally, Ref. [93] showed the investigation of the influence of strut sweep on the flowfield in an annular duct. The swept strut did not have clear advantages over the unswept strut.

The supersonic flow past a fin intersecting a flat plate is a topic reported by Hung and Buning [82], McMaster and Shang [87], Knight et al. [83], Chen and Hung [19], Lakshmanan and Tiwari [85, 86], and Zemsch and Degrez [94]. Hung and Buning [82] performed an experiment to assess the influence of the thickness of the incoming boundary layer on the hoseshoe vortex forming at the junction. The Reynolds-averaged Navier-Stokes solutions obtained by Chen and Hung [19] on a cylinder perpendicular to a flat plate revealed that the main effect of increasing the Mach number is to produce a larger vortex structure because of the shock-wave/boundary-layer interaction. The Navier-Stokes solutions of Lakshmanan and Tiwari [85] showed that, by properly filleting the junction between a swept fin and a flat plate, it is possible to reduce significantly the vorticity in the horseshoe vortex. The work of Lakshmanan and Tiwari [86] with a thin-layer Navier-Stokes solver highlighted the influence of the Mach number and the Reynolds number on the number of vortices at the wing-body junction. McMaster and Shang [87] solved the Navier-Stokes equations to determine the variations in the flowfield when the sweepback angle of the fin is changed. The interference between the fin and the flat wall was measured by Zemsch and Degrez [94] using laserDoppler velocimetry (LDV) and computed with conical Euler equations. The experiment 
showed separation on the leeward side of the fin when the sweep angle and the incidence was large.

One application of the study of the flow around a fin perpendicular to a flat wall is the design of wing-fuselage junctions. Favorable aerodynamic interference was achieved between the wing and the fuselage by Pritulo et al. [89]. The shape of the wing-body combination was determined by employing an Euler flow solver and applying simple surface deformations to the existing geometry.

\subsection{Contributions to the Field}

Although the literature is abundant on the topic of interference, only few studies propose a simple method or an equation to estimate the interference drag. In subsonic flow, Hoerner [24] took into account several geometric parameters and obtained trends and equations for evaluating the drag penalty of a strut-wall junction. The purpose of this dissertation is to extend Hoerner's general approach to the transonic flow regime. But instead of performing wind tunnel tests, numerical computations on unstructured grids are employed. The objective is to obtain "empirical" equations that describe the behavior of the interference drag in terms of a few geometric parameters. These can then be included in the MDO capability. Along the way, a better understanding of the phenomena taking place near strut-wall and wing-strut junctions in transonic flow will be gained. 


\section{Chapter 3}

\section{Description of the Computational Fluid Dynamics Tools}

The analysis of the flowfield with Computational Fluid Dynamics (CFD) requires several steps that are equally important: the geometry definition, the grid generation, the flow solution, and the analysis of the results.

The geometry of the configuration of interest has to be defined with enough detail in regions where a large concentration of mesh points will be required, because this will directly impact the mesh quality. Based on the geometry at hand, the size of the computational domain used in the analysis is determined. For the calculations made in transonic flow about a wing, the boundaries of the computational box typically extend 10 chords to the far-field downstream [73, 95].

The grid point distribution is specified throughout the domain with a concentration of mesh points in the regions where strong flow gradients are expected to be found. More points allow for a better resolution of the properties in those regions. Once the grid spacing is specified, the computational domain is filled with grid cells and the boundary conditions are applied.

The flow solver uses the cells to compute an approximation to the flow equations. This is an iterative process that stops when a given convergence criterion is met, typically a decrement of the residual. The solution is studied along cross sections and on the body surface to assess the quality of the solution and the underlying grid. Integrated quantities such as lift and drag are also considered in this process. If the results don't meet the 
expectations in terms of accuracy, the grid is improved in the regions that need it and the flow equations are solved again. This continues until the results are judged satisfactory.

In this chapter, two of the steps described before will be discussed. First, the unstructured grid generation process used in this work will be presented. Then, the flow solver will be described to complete the picture.

\subsection{Unstructured Grid Generation}

Several approaches can be used to generate grids for CFD computations. A discussion of the most widely used methods is presented by Thompson et al. [96] and Mavriplis [97]. In the present work, the governing flow equations were solved on unstructured grids because the unstructured grid methodology provides a lot of flexibility in analyzing complex geometries. In the dissertation, the term inviscid grid refers to a grid suitable for inviscid, Euler flow calculations. This is opposed to a viscous grid, which is characterized by fine mesh spacing in the direction normal to viscous surfaces to resolve the boundary layer. A viscous grid is employed for Navier-Stokes computations. For two-dimensional applications, the code AFLR2 $[98,99]$ was used to generate triangular elements with the advancing-front/localreconnection procedure. Three-dimensional problems were solved on tetrahedral grid cells obtained with VGRIDns $[100,101]$ using the advancing-front/advancing-layers method. These codes are well-tested and have been employed successfully by others for comparable studies. An overview of the methods implemented in those codes is presented in this section.

\subsubsection{Two-Dimensional Grids}

\section{Grids for Inviscid Calculations}

To obtain an inviscid grid with AFLR2, the grid spacing is prescribed on the bodies and on the far-field boundary. Based on the grid point distribution on the boundaries, a field function is determined. That function represents the grid spacing at any point in the flowfield. The curves on the bodies and on the far-field boundary are then discretized into edges to form the initial front of the mesh. One at a time, new points are added to the field and connected to the edges of the front. The position of the newly created points is based on the field function. The front evolves as new triangles are formed. The process of point insertion stops 
when the computational domain is completely discretized in triangles. After this initial node connectivity is obtained, the grid quality is improved by reconnecting the points based on the min-max quality criterion. This criterion consists in minimizing the maximum angle within each cell of the mesh.

\section{Grids for Viscous Calculations}

For high Reynolds number viscous computations [99], highly stretched grids are employed to resolve the thin boundary layer on the bodies. Viscous layers are generated in a direction normal to the viscous surfaces in a semi-structured fashion. The thickness of the first layer and the growth rate of subsequent viscous layers are specified. This determines the point distribution along the normal direction. Two types of normals are used to generate the viscous layers. The first one consists in computing the normal to each boundary face of the mesh. The second one is obtained by smoothing the first set of normals. To improve the mesh quality at sharp corners such as at the trailing edge of an airfoil, more normals are added in those regions. At first, the nodes are placed along the direction specified by the unsmoothed normal. The subsequent layers are generated along the normal determined by a weighted-average of the smoothed and the unsmoothed normals. The creation of viscous layers continues until one of the following stopping criteria is met:

- The grid spacing of the viscous layer corresponds to the one prescribed for the inviscid part of the grid;

- Viscous layers from different surfaces collide;

- A point is attempted to be put too close to an existing point, based on a tolerance specified within AFLR2.

The grid generation then switches to the advancing-front point placement scheme described above for inviscid grids. 


\subsubsection{Three-Dimensional Grids}

\section{Grids for Inviscid Calculations}

For 3-D applications, several steps are required to obtain the volume grid. Figure 3.1 depicts a flowchart of the grid generation process. The code GridTool [102] is utilized to define 3- and 4-sided patches to cover the geometry and the boundary faces of the computational domain. Patches are similar to the block faces employed with structured grids. They are required to discretize the geometry and let the grid generator know how the various elements of the geometry are connected together. These patches will later be triangulated individually by VGRIDns. The shape of the patch has a strong influence on the resulting triangulation. Square patches generally provide the best surface mesh. Since it is not always possible to create such patches, it is advisable to try to create low aspect ratio patches with at least two parallel edges. Patches with highly curved edges should be avoided as much as possible. Instead, they should be broken down into several smaller triangular or rectangular patches. In order for VGRIDns to triangulate a patch, it maps it to a 2-D equivalent triangular or rectangular surface. After the surface is triangulated, it transforms it back to its original shape. Highly distorted surface triangulation generally arises from the fact that the original and the transformed versions of the patch are too different from each other. Efforts spent early on in the definition of "good" patches pay off later in the process. An example of the patch definition on a wing planform is shown in Figure 3.2. The patches are almost rectangular along the span. Near the leading edge of the wing, more patches could be employed if necessary. At the wing tip, smaller patches are employed to provide a better control over the mesh quality in that region.

Once the patches are defined through the entire computational domain, the grid point distribution needs to be specified with GridTool. Unlike the 2-D grid generation code AFLR2, the grid spacing is not specified directly along the body surface or in the far field. It is achieved through the use of either linear or nodal sources located inside the geometry and in the flowfield. These sources act like heat sources. In the vicinity of a heat source, the temperature is very close to the temperature of the heat source. As we move away from the heat source, the temperature changes gradually towards its freestream value. The same is true for a grid source. Close to the grid source, the spacing is the same as the one specified by the source itself. As we get farther away from the source, the grid becomes coarser. For each grid source, several parameters can be controlled, the most important ones being: 


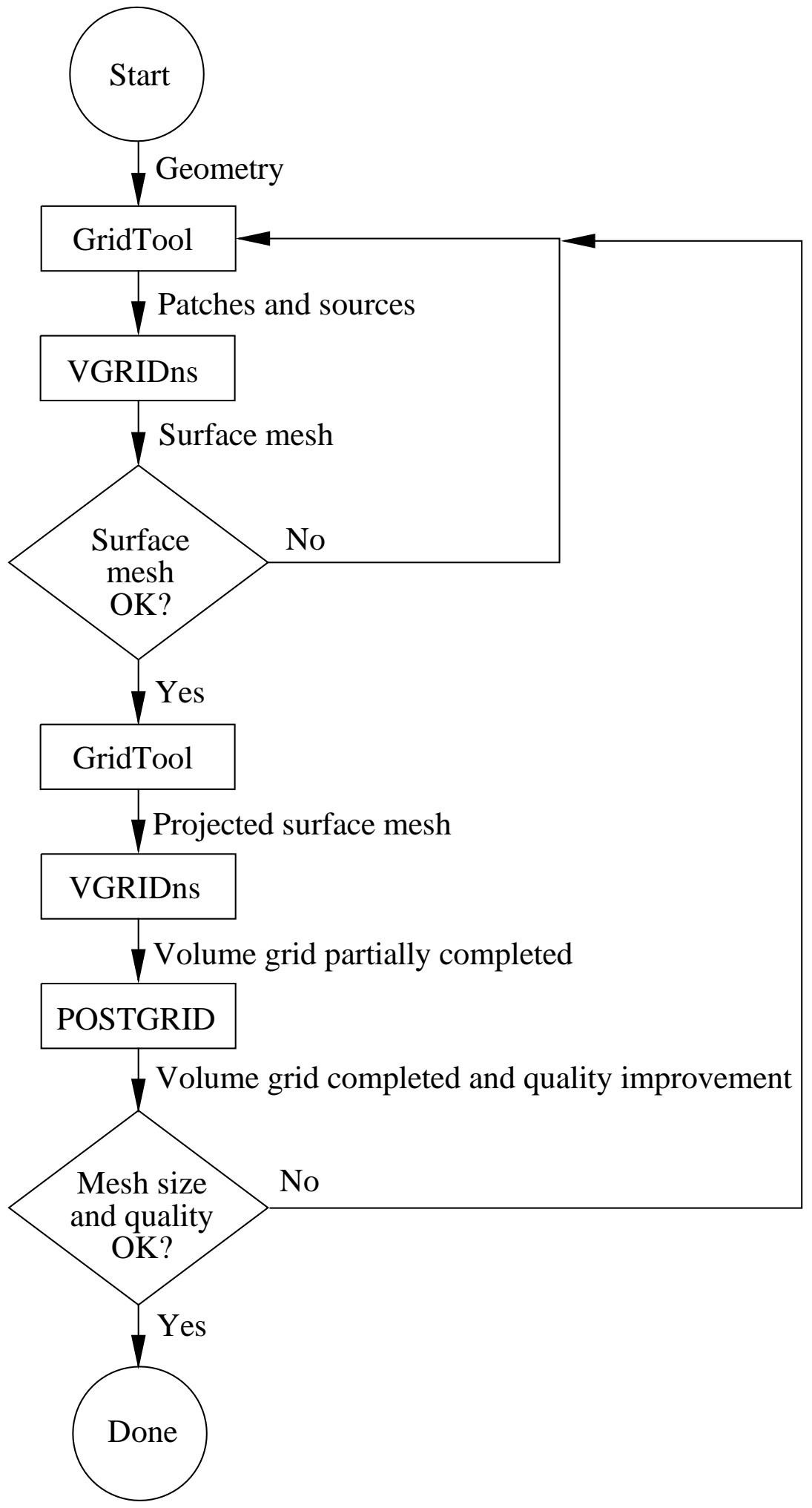

Figure 3.1: Flowchart of the grid generation process in three dimensions 


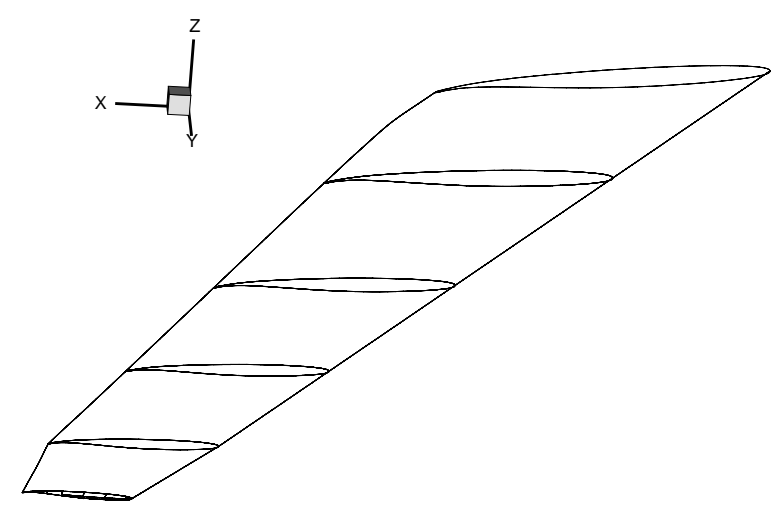

(a)

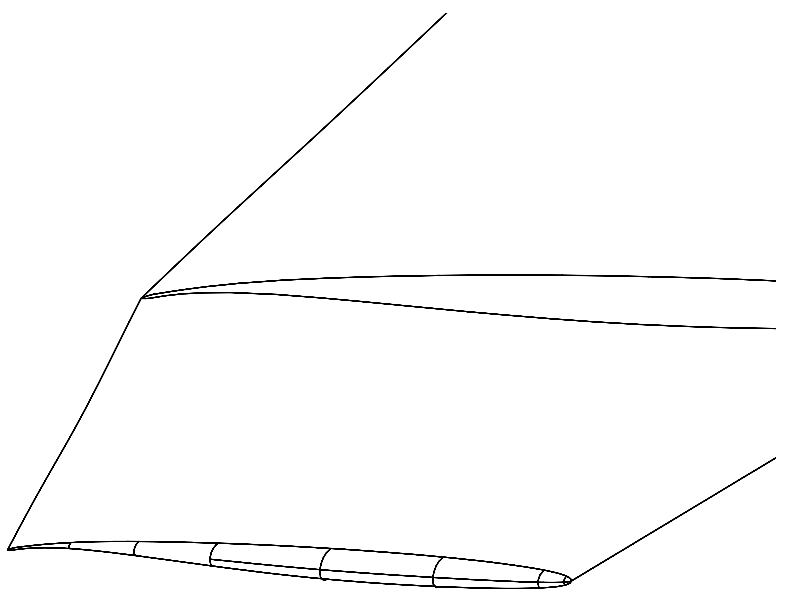

(b)

Figure 3.2: Patch definition on a wing planform (a) View of the planform (b) Close-up view of the wing tip

- Grid spacing $s$ of the source;

- Stretching $S$ of the source;

- Strength or intensity $a_{n}$ of the source.

The grid spacing $s$ specifies how small the tetrahedra will be next to the source while the stretching parameter $S$ controls the aspect ratio of the tetrahedra. It is important to bear in mind that the effect of a grid source is intimately related to its strength $a_{n}$. When $a_{n}$ is reduced, the grid spacing will become coarser very quickly away from the source. The opposite happens following an increase in $a_{n}$. Therefore, the $a_{n}$ of each source has to be adjusted relative to the others in order to obtain the proper grid point distribution. The spatial variation of the grid parameters is obtained by solving a Poisson equation based on the sources distributed in the field.

To obtain a satisfactory unstructured grid, the linear sources are usually placed inside the geometry of interest to allow control over the grid distribution in the vicinity of the body. As shown in Figure 3.3, linear sources are used at the leading edge and at the trailing edge of the wing to enforce the grid spacing there. Stretching can be employed along directions of small gradients, such as along the spanwise direction of a straight wing. In the far-field, nodal "corner" sources are located at each corner of the computational box to specify the 


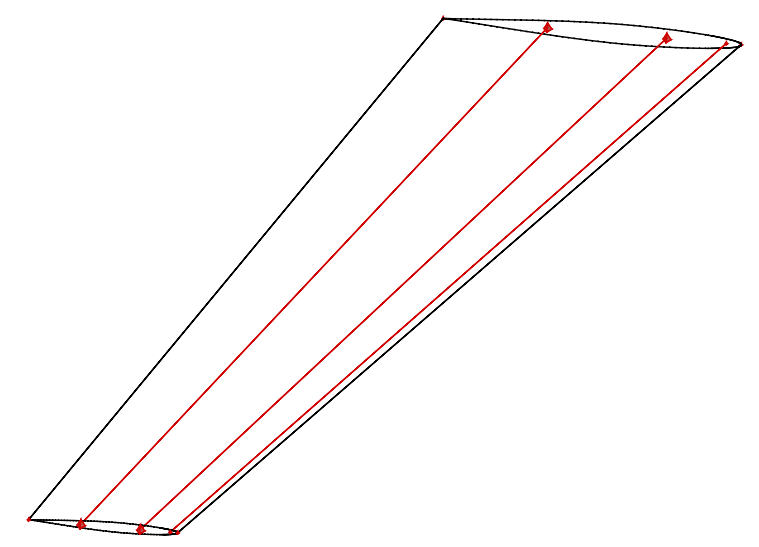

Figure 3.3: Five linear sources located inside the wing

grid spacing there as shown in Figure 3.4. A reduction of the spacing $s$ or an increase of the strength $a_{n}$ of the corner sources is accompanied by an increase in the number of tetrahedral cells in the mesh. A larger spacing $s$ or a smaller strength $a_{n}$ of the corner sources leads to a reduction of the number of cells in the mesh. In some instances, this can even prevent the completion of the volume grid generation.

VGRIDns is then utilized to determine the grid point spacing in the field and to triangulate each patch of the geometry. If no stretching is employed anywhere in the mesh, VGRIDns provides a quality assessment of the surface grid based on the deviation of each triangle from acceptable limits. Highly skewed triangles are flagged. Abrupt changes in specified grid spacing and poorly adjusted source strengths often lead to numerous of those skewed triangles. It is possible to change the orientation of the patches to obtain a surface mesh containing fewer or no skewed surface triangles. However, in most instances, the triangulation in the problematic regions must be improved by going back to GridTool to perform one of the following:

- Change $s, a_{n}$, or the location of some grid sources;

- Re-define some patches or break existing patches into smaller ones.

The grid generation code VGRIDns is then run to obtain a new surface mesh. It may be necessary to go back and forth several times between GridTool and VGRIDns in order to 


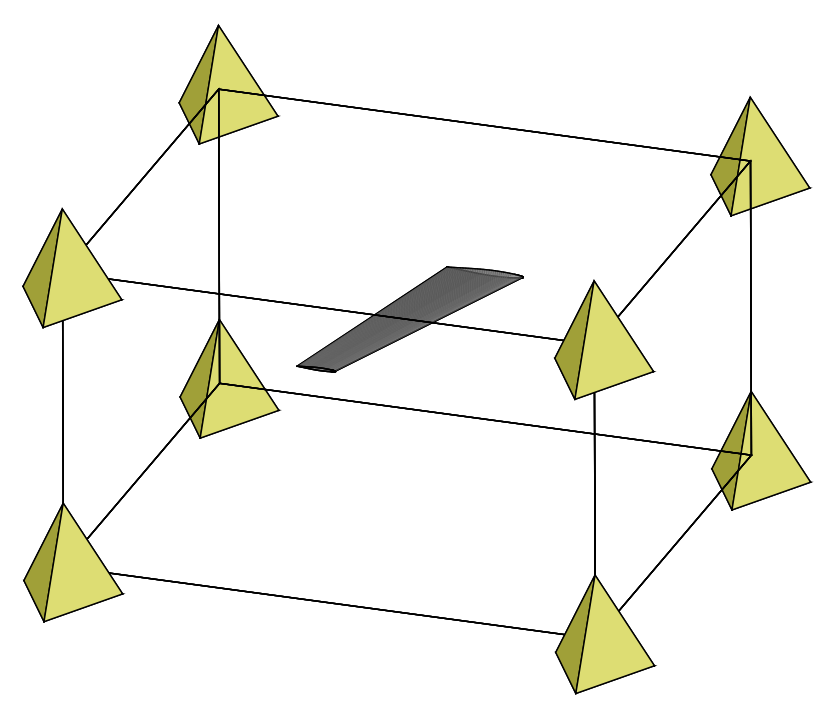

Figure 3.4: Wing and nodal sources located at the corners of the computational box

obtain a satisfactory mesh. The specification of the source parameters is sometimes more an art than a science. This is because the spacing can't be specified directly as in AFLR2. It requires the careful adjustment of the source parameters with respect to one another.

The quality of the surface grid is determined visually using the code VGRIDns for unstretched meshes only. If no stretching is employed in the mesh, there should ideally be no skewed triangles flagged by VGRIDns on the surface. However, if stretching is utilized, the option for mesh quality assessment in VGRIDns is automatically turned off to avoid flagging the vast majority of surface triangles. Generally, it is preferrable to generate an unstretched mesh, make sure there is no skewed surface triangle, and generate a new grid with stretching along directions of small gradients only. Once a satisfactory surface grid is obtained, the mesh needs to be projected onto the original geometry to ensure that the triangles lie perfectly on the surface of the body. This is especially true when dealing with sharp edges or with supercritical airfoil sections. In the latter case, the mesh on the upper and lower surfaces could cross in the region near the trailing edge. In general, there is very little movement of the mesh as a result of the projection.

The projected mesh is fed back into VGRIDns to obtain the volume grid. The surface mesh made of triangles is used as the initial front for the grid generation. One by one, nodes are inserted in the field based on the prescribed grid point distribution. The newly inserted point is connected to the triangle on the front and a tetrahedral cell is formed. As more 
cells are formed, the front evolves until it collapses onto itself. When no more cells can be formed in the computational domain, voids or empty pockets can exist. It is then necessary to use the grid post-processor POSTGRID to complete the volume mesh by local remeshing. Layers of cells surrounding the voids are removed and attempts are made at completing the mesh starting from that front. For the case of a wing intersecting a flat wall, regions near the wing root or near the trailing edge are prone to the formation of voids.

Once the volume grid is completed, a grid quality assessment is conducted for all the tetrahedra of the mesh. Cells that don't meet a given quality criterion because of their aspect ratio or their skewness are identified. It is then possible to improve the overall mesh quality through local remeshing or mesh smoothing. The former removes some layers of the grid and employs the local remeshing technique described above. The latter consists in

picturing the edges of the mesh as springs. The nodes of the mesh are then moved with respect to one another in order for the mesh quality to be improved in that region. If no stretching were employed in the mesh, this would correspond to trying to get the tetrahedral cells to be as close as possible to equilateral.

\section{Grids for Viscous Calculations}

For viscous flow calculations, highly stretched cells are required near the viscous surfaces in order to resolve the boundary layer. The viscous layers of cells are generated with the advancing-layers method. Each layer of tetrahedral cells emanates in a direction normal to the viscous surface in a semistructured fashion. Each newly generated layer of cells is stacked on top of the previous one. The normal is computed at viscous boundary faces of the surface mesh. VGRIDns doesn't add more normals when a sharp corner is detected as opposed to AFLR2. The grid obtained in the vicinity of the trailing edge of an airfoil using AFLR2 and VGRIDns is shown in Figure 3.5. It can be seen that AFLR2 tends to grow the viscous layers perpendicularly to the surface even very close to the trailing edge. On the other hand, VGRIDns blends out the existing normals to ensure a smooth transition between the upper and lower surface of the airfoil.

VGRIDns controls the thickness of the $i^{t h}$ viscous layer of cells using the following expression [101]:

$$
\delta_{i}=\delta_{1}\left[1+r_{1}\left(1+r_{2}\right)^{i-1}\right]^{i-1}
$$




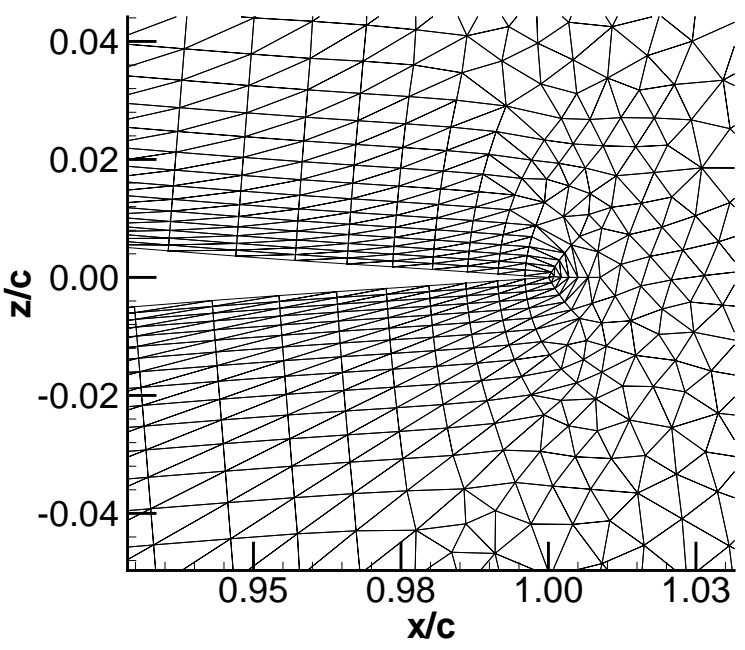

(a)

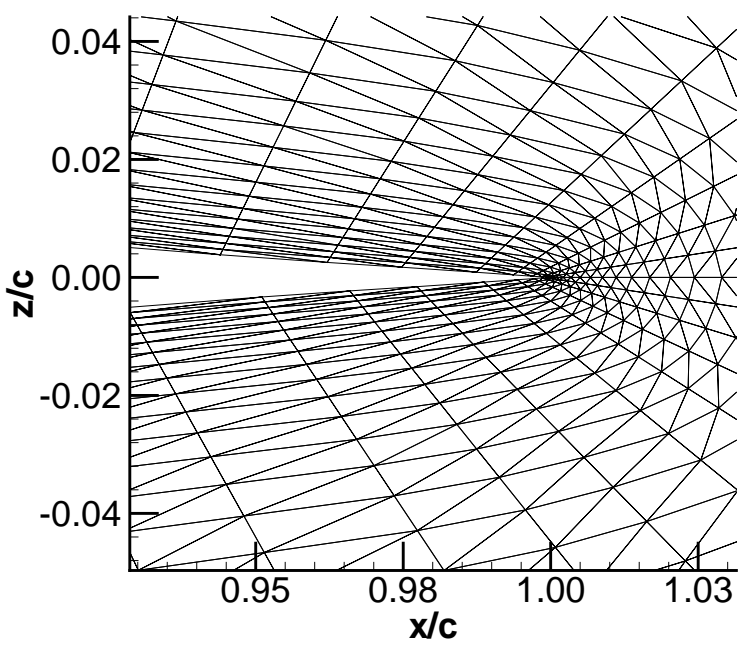

(b)

Figure 3.5: Influence of the normals on the growth of the viscous layers around a sharp corner (a) Addition of normals (AFLR2) (b) Use of existing normals only (VGRIDns)

where $\delta_{1}$ is the normal grid spacing at the wall, while $r_{1}$ and $r_{2}$ represent the grid growth rates. The distance $\delta_{1}$ has to be chosen in order to have at least one point in the laminar sublayer of the boundary layer for turbulent flow calculations. The distance $\delta_{1}$ is approximated using the relationships for a turbulent boundary layer over a flat plate. After the CFD solution is obtained, it is important to verify that the first grid point above the body surface is located within the laminar sublayer of the turbulent boundary layer. Until that condition is met, new grids have to be generated with gradually smaller values of $\delta_{1}$. For full Navier-Stokes calculations in which the boundary layer is resolved all the way to the wall without the use of wall function,

$$
0.2 \leq r_{1} \leq 0.4
$$

and

$$
r_{2}=0
$$

are typically used [103]. If wall functions are used instead,

$$
0.4 \leq r_{1} \leq 0.9
$$

and

$$
0.04 \leq r_{2} \leq 0.08
$$

provide a good resolution of the boundary layer [103]. 
The viscous layers are stacked on top of each other until a stopping criterion is met. If two fronts of viscous layers merge or if the local cell aspect ratio approaches unity, the growth of viscous layers stops locally. The layers continue to grow on other fronts until the stopping criterion is met everywhere. From then on, the grid generation is performed with the advancing-front methodology, starting with the final viscous layers as the initial front. The volume grid generation continues as described above for inviscid meshes. The grid can be completed in the same fashion. However, grid smoothing should be avoided as a means of improving the mesh quality. It deteriorates the volume grid and leads to the formation of cells with negative volume.

\subsection{Flow Solver}

In this section, the flow solvers FUN2D and FUN3D $[104,105]$ used for this work are described in detail. These codes were chosen because our studies were conducted for NASA Langley, so close cooperation was facilitated. The material presented here is taken in large part from the work published by Anderson and Bonhaus [104]. The reader is encouraged to consult this reference for a more complete discussion of the flow solver and the algorithm behind it.

\subsubsection{Governing Equations}

The flow solvers FUN2D and FUN3D used in this work solve the two- and three-dimensional Reynolds-averaged Navier-Stokes equations respectively. In this section, the three-dimensional version of the equations will be presented. The equations are non-dimensionalized [104] with respect to the freestream density $\tilde{\rho}_{\infty}$, speed of sound $\tilde{a}_{\infty}$, temperature $\tilde{T}_{\infty}$, viscosity $\tilde{\mu}_{\infty}$, thermal conductivity $\tilde{k}_{\infty}$, and a reference length $L$. They can be written as:

$$
V \frac{\partial \mathbf{Q}}{\partial t}+\oint_{\partial \Omega}\left(\overrightarrow{\mathbf{F}}_{\mathbf{i}} \cdot \hat{\mathbf{n}}\right) d S-\oint_{\partial \Omega}\left(\overrightarrow{\mathbf{F}}_{\mathbf{v}} \cdot \hat{\mathbf{n}}\right) d S=0
$$

where $V$ is the volume of the control volume, $\partial \Omega$ is the surface bounding the control volume and $\hat{\mathbf{n}}$ is the outward pointing normal of the control volume boundary. The vector of 
conserved variable, $\mathbf{Q}$, is defined as

$$
\mathbf{Q}=\left\{\begin{array}{c}
\rho \\
\rho u \\
\rho v \\
\rho w \\
E
\end{array}\right\}
$$

where $\rho$ is the density, $u, v, w$ are the Cartesian velocity components, and $E$ is the total energy per unit volume. The inviscid fluxes, $\overrightarrow{\mathbf{F}}_{\mathbf{i}}$, are given by

$$
\vec{F}_{\mathbf{i}}=\mathbf{f} \overrightarrow{\mathbf{i}}+\mathrm{g} \overrightarrow{\mathbf{j}}+\mathbf{h} \overrightarrow{\mathbf{k}}
$$

where

$$
\mathbf{f}=\left\{\begin{array}{c}
\rho u \\
\rho u^{2}+p \\
\rho u v \\
\rho u w \\
(E+p) u
\end{array}\right\}, \mathbf{g}=\left\{\begin{array}{c}
\rho v \\
\rho v u \\
\rho v^{2}+p \\
\rho v w \\
(E+p) v
\end{array}\right\} \text {, and } \mathbf{h}=\left\{\begin{array}{c}
\rho w \\
\rho w u \\
\rho w v \\
\rho w^{2}+p \\
(E+p) w
\end{array}\right\}
$$

where $p$ is the pressure. The viscous flux vector, $\overrightarrow{\mathbf{F}}_{\mathbf{v}}$, is expressed as

$$
\overrightarrow{\mathbf{F}}_{\mathrm{v}}=\mathbf{f}_{\mathrm{v}} \overrightarrow{\mathbf{i}}+\mathbf{g}_{\mathrm{v}} \overrightarrow{\mathbf{j}}+\mathbf{h}_{\mathrm{v}} \overrightarrow{\mathbf{k}}
$$

where

$$
\begin{gathered}
\mathbf{f}_{\mathbf{v}}=\left\{\begin{array}{c}
0 \\
\tau_{x x} \\
\tau_{x y} \\
\tau_{x z} \\
u \tau_{x x}+v \tau_{y x}+w \tau_{z x}-q_{x}
\end{array}\right\} \\
\mathbf{g}_{\mathbf{v}}=\left\{\begin{array}{c}
0 \\
\tau_{y x} \\
\tau_{y y} \\
\tau_{y z} \\
v \tau_{y y}+w \tau_{z y}-q_{y}
\end{array}\right\} \\
\mathbf{h}_{\mathbf{v}}=\left\{\begin{array}{c}
u \\
\tau_{z x} \\
\tau_{z y} \\
\tau_{z z} \\
+v \tau_{y z}+w \tau_{z z}-q_{z}
\end{array}\right\}
\end{gathered}
$$


The viscous stresses $\tau_{x x}, \tau_{y y}, \tau_{z z}, \tau_{x y}, \tau_{y x}, \tau_{x z}, \tau_{z x}, \tau_{y z}$, and $\tau_{z y}$ can be written as

$$
\begin{gathered}
\tau_{x x}=\left(\mu+\mu_{t}\right) \frac{M_{\infty}}{R e} \frac{2}{3}\left[2 \frac{\partial u}{\partial x}-\left(\frac{\partial v}{\partial y}+\frac{\partial w}{\partial z}\right)\right] \\
\tau_{y y}=\left(\mu+\mu_{t}\right) \frac{M_{\infty}}{R e} \frac{2}{3}\left[2 \frac{\partial v}{\partial y}-\left(\frac{\partial u}{\partial x}+\frac{\partial w}{\partial z}\right)\right] \\
\tau_{z z}=\left(\mu+\mu_{t}\right) \frac{M_{\infty}}{R e} \frac{2}{3}\left[2 \frac{\partial w}{\partial z}-\left(\frac{\partial u}{\partial x}+\frac{\partial v}{\partial y}\right)\right] \\
\tau_{x y}=\tau_{y x}=\left(\mu+\mu_{t}\right) \frac{M_{\infty}}{R e}\left(\frac{\partial u}{\partial y}+\frac{\partial v}{\partial x}\right) \\
\tau_{x z}=\tau_{z x}=\left(\mu+\mu_{t}\right) \frac{M_{\infty}}{R e}\left(\frac{\partial u}{\partial z}+\frac{\partial w}{\partial x}\right) \\
\tau_{y z}=\tau_{z y}=\left(\mu+\mu_{t}\right) \frac{M_{\infty}}{R e}\left(\frac{\partial v}{\partial z}+\frac{\partial w}{\partial y}\right)
\end{gathered}
$$

The heat flux terms $q_{x}, q_{y}$, and $q_{z}$ are expressed as

$$
\begin{aligned}
& q_{x}=-\frac{M_{\infty}}{R e(\gamma-1)}\left(\frac{\mu}{P r}+\frac{\mu_{t}}{P r_{t}}\right) \frac{\partial a^{2}}{\partial x} \\
& q_{y}=-\frac{M_{\infty}}{\operatorname{Re}(\gamma-1)}\left(\frac{\mu}{P r}+\frac{\mu_{t}}{P r_{t}}\right) \frac{\partial a^{2}}{\partial y} \\
& q_{z}=-\frac{M_{\infty}}{\operatorname{Re}(\gamma-1)}\left(\frac{\mu}{P r}+\frac{\mu_{t}}{P r_{t}}\right) \frac{\partial a^{2}}{\partial z}
\end{aligned}
$$

The set of equations makes use of the equation of state for a perfect gas:

$$
p=(\gamma-1)\left[E-\rho \frac{\left(u^{2}+v^{2}+w^{2}\right)}{2}\right]
$$

and the laminar viscosity of the fluid is obtained from Sutherland's law:

$$
\mu=\frac{\hat{\mu}}{\hat{\mu}_{\infty}}=\frac{\left(1+C^{*}\right)\left(\hat{T} / \hat{T}_{\infty}\right)^{3 / 2}}{\hat{T} / \hat{T}_{\infty}+C^{*}}
$$


where Sutherland's constant, $C^{*}$, is divided by a freestream temperature assumed to be $460^{\circ} R$ to yield

$$
C^{*}=\frac{198.6}{460.0}
$$

The set of equation is completed by the determination of the turbulent viscosity $\mu_{t}$ through the solution of a partial differential equation presented in the next section. The turbulence model used is the one developed by Spalart and Allmaras [106].

In this work, results are also presented for the inviscid counterpart of the Navier-Stokes equations, namely the Euler equations. For that case, the formulation is easily obtained by setting the vector of viscous fluxes $\overrightarrow{\mathbf{F}}_{\mathbf{v}}$ to zero. There is no need to solve an additional equation for the turbulence model.

\subsubsection{Turbulence Model}

The calculations performed in this study made use of the one-equation turbulence model of Spalart and Allmaras [106] incorporated into the flow solver employed. This model is well-regarded in the aerodynamics community. It is based on:

$$
\begin{aligned}
\frac{\mathrm{D} \tilde{\nu}}{\mathrm{D} t}= & \frac{M_{\infty}}{\sigma R e}\left\{\nabla \cdot\left[\left(\nu+\left(1+c_{b_{2}}\right) \tilde{\nu}\right) \nabla \tilde{\nu}\right]-c_{b_{2}} \tilde{\nu} \nabla^{2} \tilde{\nu}\right\} \\
& -\frac{M_{\infty}}{R e}\left[c_{w_{1}} f_{w}-\frac{c_{b_{1}}}{\kappa^{2}} f_{t_{2}}\right]\left(\frac{\tilde{\nu}}{d}\right)^{2}+c_{b_{1}}\left(1-f_{t_{2}}\right) \tilde{S} \tilde{\nu}+\frac{R e}{M_{\infty}} f_{t_{1}} \Delta U^{2}
\end{aligned}
$$

where

$$
\begin{gathered}
f_{v_{1}}=\frac{\chi^{3}}{\chi^{3}+c_{v_{1}}^{3}} \\
\chi \equiv \frac{\tilde{\nu}}{\nu} \\
\tilde{S} \equiv S+\frac{M_{\infty}}{R e} \frac{\tilde{\nu}}{\kappa^{2} d^{2}} f_{v_{2}} \\
f_{v_{2}}=1-\frac{\chi}{1+\chi f_{v_{1}}}
\end{gathered}
$$


where $S$ is the magnitude of the vorticity and $d$ is the distance to the closest wall. The remaining factors are:

$$
\begin{gathered}
f_{w}=g\left[\frac{1+c_{w_{3}}^{6}}{g^{6}+c_{w_{3}}}\right]^{1 / 6} \\
g=r+c_{w_{2}}\left(r^{6}-r\right) \\
r \equiv \frac{M_{\infty}}{R e} \frac{\tilde{\nu}}{\tilde{S} \kappa^{2} d^{2}}
\end{gathered}
$$

The transition location is controlled through the last term of Eq. 3.22 which involves the factors $f_{t_{1}}$ and $f_{t_{2}}$. All the analyses presented in the dissertation were performed for fully turbulent flow and thus, the expressions for those variables are not provided here. The value of the constants $c_{b_{1}}, c_{b_{2}}, c_{v_{1}}, c_{w_{1}}, c_{w_{2}}$, and $c_{w_{3}}$ were omitted for the sake of conciseness. They are explained in a detailed fashion in Ref. [106].

Eq. 3.22 is solved for $\tilde{\nu}$ and the eddy viscosity is then calculated from

$$
\mu_{t}=\rho \nu_{t}=\rho \tilde{\nu} f_{v_{1}}
$$

The turbulent viscosity $\mu_{t}$ is then used to determine the viscous stresses and the heat flux terms described in Eqs. 3.10-3.18.

\subsubsection{Finite Volume Formulation}

The concepts presented in this section are valid for 2-D and 3-D applications. In order to preserve the generality of the derivations, the equations will be established for the 3-D cases. However, some concepts may be illustrated with 2-D examples for convenience.

The Reynolds-averaged Navier-Stokes equations are solved using a finite volume formulation. The computational domain is divided into a finite number of elements, triangular for 2-D computations and tetrahedral for 3-D cases. Around each node of the mesh, a control volume is established by joining the midpoint of each edge to the centroid of the element. The way the element is partitioned is illustrated in Figures 3.6 and 3.7 for triangular and tetrahedral elements respectively. Around a given node, the individual control volumes within each cell combine to form a control volume. For 2-D meshes, this is depicted in Figure 3.8 where 


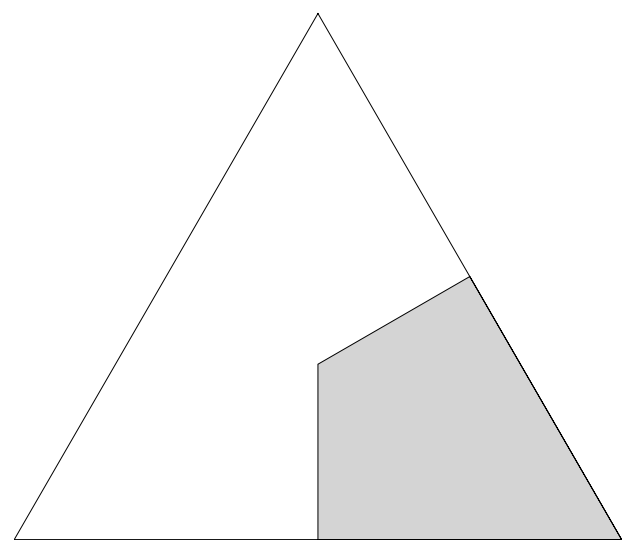

Figure 3.6: One of three individual control volumes within a triangular cell

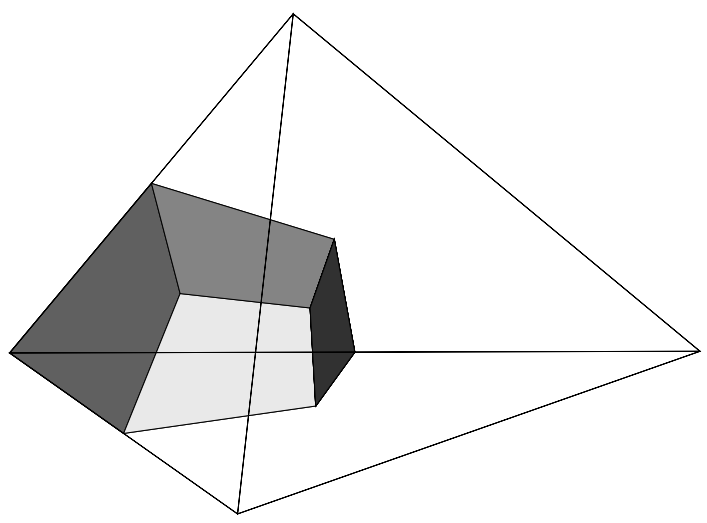

Figure 3.7: One of four individual control volumes within a tetrahedral cell 


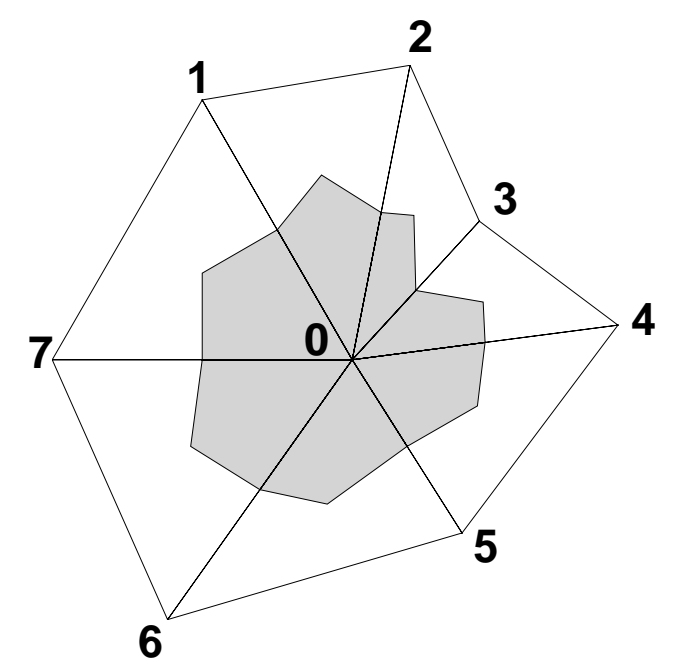

Figure 3.8: Control volume obtained by combining the individual control volumes of the neighboring cells

the central node is surrounded by a control volume made up of seven individual control volumes. By a similar procedure, a control volume can be obtained in 3-D.

The Reynold-averaged Navier-Stokes equations are discretized and integrated over each control volume of the mesh. For a given node and its surrounding control volume $V$, the discretized form of the equations can be written as:

$$
V \frac{\Delta \mathbf{Q}}{\Delta t}+\sum_{S}\left(\overrightarrow{\mathbf{F}}_{\mathbf{i}} \cdot \hat{\mathbf{n}}\right) \Delta S-\sum_{S}\left(\overrightarrow{\mathbf{F}}_{\mathbf{v}} \cdot \hat{\mathbf{n}}\right) \Delta S=0
$$

The summation of the inviscid and viscous fluxes is performed over the boundary faces $\Delta S$ of the control volume. At each node, the residual $\mathbf{R}$ is calculated by considering the flux balance terms:

$$
\mathbf{R}=\sum_{S}\left(\overrightarrow{\mathbf{F}}_{\mathbf{i}} \cdot \hat{\mathbf{n}}\right) \Delta S-\sum_{S}\left(\overrightarrow{\mathbf{F}}_{\mathbf{v}} \cdot \hat{\mathbf{n}}\right) \Delta S
$$

The contributions of the inviscid and viscous terms to the residual are calculated separately. At each face, the inviscid fluxes are evaluated using Roe's flux-difference splitting scheme [107]. This scheme provides an approximate solution to the Riemann problem in order to represent the fluxes at the control-volume face. The Roe-averaged matrix $\hat{\mathbf{A}}$ is constructed at the face based on averaged quantities for $\mathbf{Q}$. The resulting flux at the interface becomes:

$$
\overrightarrow{\mathbf{F}}_{\mathbf{i}}=\frac{1}{2}\left\{\left(\overrightarrow{\mathbf{F}}_{\mathbf{i}}\left(\mathbf{Q}_{\mathbf{R}}\right)+\overrightarrow{\mathbf{F}}_{\mathbf{i}}\left(\mathbf{Q}_{\mathbf{L}}\right)\right)-|\hat{\mathbf{A}}|\left(\mathbf{Q}_{\mathbf{R}}-\mathbf{Q}_{\mathbf{L}}\right)\right\}
$$


where $\mathbf{Q}_{\mathbf{R}}$ and $\mathbf{Q}_{\mathbf{L}}$ denote the data on the right and left side of the face respectively. Although $\mathbf{Q}_{\mathbf{R}}$ and $\mathbf{Q}_{\mathbf{L}}$ may be discontinuous at the face, Roe's scheme ensures that the fluxes will be continuous across it. The determination of the node on the left and right side of a face is based on the orientation of the normal to the face. The normal is defined in such a way that it points from the left side to the right side of the face. When calculating the contribution from the inviscid fluxes to the residual in Eq. 3.32, a positive contribution $\left(\overrightarrow{\mathbf{F}}_{\mathbf{i}} \cdot \hat{\mathbf{n}}\right) \Delta S$ is added to the control volume on the left and an equivalent negative contribution $\left(\overrightarrow{\mathbf{F}}_{\mathbf{i}} \cdot(-\hat{\mathbf{n}})\right) \Delta S$ is added to the control volume on the right.

The flow solver allows the use of first- or second-order accurate differencing. For firstorder accurate, this means that the value of $\mathbf{Q}_{\mathbf{R}}$ and $\mathbf{Q}_{\mathbf{L}}$ are taken from the node lying on either side of the face. It doesn't make use of the data from a series of neighboring nodes. Figure 3.9 depicts the control volume surrounding node 0 in 2-D with the left and right normals $\hat{\mathbf{n}}_{\mathbf{L} 2}$ and $\hat{\mathbf{n}}_{\mathbf{R} 2}$ associated with edge 0-2. The normals for the other edges are analogously defined. In determining the flux contributions for the faces whose normals are $\hat{\mathbf{n}}_{\mathbf{L} 2}$ and $\hat{\mathbf{n}}_{\mathbf{R} 2}$, we take:

$$
\begin{aligned}
\mathrm{Q}_{\mathrm{L}} & =\mathrm{Q}_{0} \\
\mathrm{Q}_{\mathrm{R}} & =\mathrm{Q}_{2}
\end{aligned}
$$

This procedure is equivalent to considering a Taylor series expansion that keeps only the leading term, thus producing an approximation with a first-order truncation error.

For second-order accurate differencing, the neighboring nodes are considered in the determination of the $\mathbf{Q}$ on either side of the face. It uses a Taylor series expansion where one more term is kept so that:

$$
\mathrm{Q}_{\mathrm{face}}=\mathrm{Q}_{\mathbf{0}}+\nabla \mathrm{Q} \cdot \overrightarrow{\mathbf{r}}
$$

where $\overrightarrow{\mathbf{r}}$ is the vector joining the node of interest to the middle of the cell edge. This is illustrated in Figure 3.9 for the case discussed before. This leads to:

$$
\begin{aligned}
\mathrm{Q}_{\mathrm{L}} & =\mathrm{Q}_{\mathbf{0}}+\nabla \mathrm{Q} \cdot \overrightarrow{\mathbf{r}} \\
\mathrm{Q}_{\mathbf{R}} & =\mathrm{Q}_{2}+\nabla \mathrm{Q} \cdot(-\overrightarrow{\mathbf{r}})
\end{aligned}
$$

First-order accurate differencing is typically used in the first iterations of the numerical procedure to get a first cut at the solution. The second-order differencing yields much more accurate results and is thus used in order to obtain the final flow solution. However, to find 


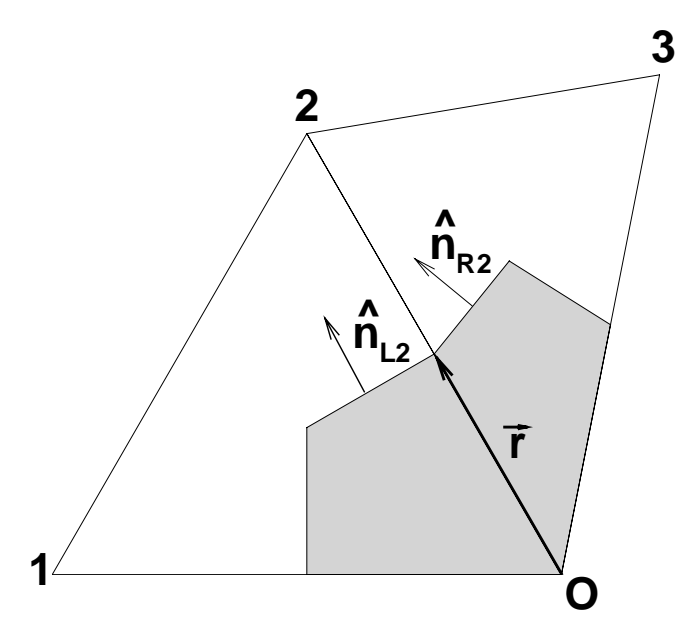

Figure 3.9: Control volume and the associated surface normals

$\mathbf{Q}_{\mathbf{R}}$ and $\mathbf{Q}_{\mathbf{L}}$, the determination of the gradient $\nabla \mathbf{Q}$ is required. This can be computed with Green's theorem or with a least-squares procedure. Anderson and Bonhaus [104] note that a least-squares procedure generally gives a better representation of the data on stretched grids. Green's theorem is used when exact representation of the gradients is required, such as when computing the viscous flux terms. This will be discussed later in this section.

The least-squares procedure is established based on the information at the nodes connecting to the node of interest. It is assumed that the data varies linearly along each edge. If we knew what $\nabla \mathbf{Q}$ is at node 0 , we could use a truncated Taylor series about that node to determine $\mathbf{Q}$ at a neighboring node $i$ with:

$$
\mathrm{Q}_{\mathbf{i}}=\mathrm{Q}_{\mathbf{0}}+\nabla \mathbf{Q} \cdot\left(\overrightarrow{\mathbf{r}_{\mathbf{i}}}-\overrightarrow{\mathbf{r}_{0}}\right)
$$

where $\overrightarrow{\mathbf{r}_{\mathbf{0}}}$ and $\overrightarrow{\mathbf{r}_{\mathbf{i}}}$ are the position vectors of nodes 0 and $i$ :

$$
\begin{aligned}
\overrightarrow{\mathbf{r}_{\mathbf{i}}}-\overrightarrow{\mathbf{r}_{\mathbf{0}}} & =\left(x_{i}-x_{0}\right) \overrightarrow{\mathbf{i}}+\left(y_{i}-y_{0}\right) \overrightarrow{\mathbf{j}}+\left(z_{i}-z_{0}\right) \overrightarrow{\mathbf{k}} \\
& =\Delta x_{i} \overrightarrow{\mathbf{i}}+\Delta y_{i} \overrightarrow{\mathbf{j}}+\Delta z_{i} \overrightarrow{\mathbf{k}}
\end{aligned}
$$

In our case, we don't know what $\nabla \mathbf{Q}$ is. But we know $\mathbf{Q}$ at the $N$ nodes connecting to node 0 . We can therefore establish a system of $N \times 3$ equations similar in form to Eq. 3.37 to calculate the gradient $\nabla \mathbf{Q}$ at node 0 based on the information stored at the neighboring 
nodes:

$$
\left[\begin{array}{ccc}
\Delta x_{1} & \Delta y_{1} & \Delta z_{1} \\
\Delta x_{2} & \Delta y_{2} & \Delta z_{2} \\
\vdots & \vdots & \vdots \\
\Delta x_{N} & \Delta y_{N} & \Delta z_{N}
\end{array}\right]\left(\begin{array}{c}
\left.\frac{\partial \mathbf{Q}}{\partial x}\right|_{0} \\
\left.\frac{\partial \mathbf{Q}}{\partial y}\right|_{0} \\
\left.\frac{\partial \mathbf{Q}}{\partial z}\right|_{0}
\end{array}\right)=\left(\begin{array}{c}
\mathbf{Q}_{\mathbf{1}}-\mathbf{Q}_{\mathbf{0}} \\
\mathbf{Q}_{\mathbf{2}}-\mathbf{Q}_{\mathbf{0}} \\
\vdots \\
\mathbf{Q}_{\mathbf{N}}-\mathbf{Q}_{\mathbf{0}}
\end{array}\right)
$$

This overdetermined system of equations is solved with the Gram-Schmidt procedure in order to preserve the accuracy of the gradients even on highly stretched meshes.

We have completed the description of the procedure used in order to compute the inviscid contribution to the residual. We will now discuss the calculation of the viscous contributions. The viscous stresses in Eqs. 3.10-3.15 contain partial derivatives of the velocity components. For example, for $\tau_{x x}$, we have:

$$
\tau_{x x}=\left(\mu+\mu_{t}\right) \frac{M_{\infty}}{R e} \frac{2}{3}\left[2 \frac{\partial u}{\partial x}-\left(\frac{\partial v}{\partial y}+\frac{\partial w}{\partial z}\right)\right]
$$

Those derivatives are evaluated with Green's theorem [107]. That theorem relates the gradient of a function $\phi$ in a volume $\Omega$ to the value of the function along the boundary $\partial \Omega$. It is valid if the function $\phi$ and its partial derivatives are continuous inside the region $\Omega$. Green's theorem can be written as:

$$
\int_{\Omega} \nabla \phi d V=\oint_{\partial \Omega} \phi \hat{\mathbf{n}} d S
$$

This theorem can be applied in a discrete way to calculate the gradient in a cell:

$$
\nabla \phi=\frac{1}{V} \sum_{S} \phi \hat{\mathbf{n}} \Delta S
$$

In the flow solver, the gradient of the velocity is assumed constant within each grid cell and is calculated with Green's theorem. For terms such as $\left(\mu+\mu_{t}\right)$ and $u\left(\mu+\mu_{t}\right)$ that appear in $\overrightarrow{\mathbf{F}}_{\mathbf{v}}$, they are computed as the average of the nodal values. The terms are then combined with the velocity gradients determined with Green's theorem to obtain the viscous contribution to the residual.

The equation for the turbulence model Eq. 3.22 is solved in a finite-volume approach in the same way as the Reynolds-averaged Navier-Stokes equations. The use of positive operators and M-type matrices [108] in the solution of the turbulence equation ensures the positivity of the eddy viscosity $\mu_{t}$. When a positive operator is applied to a vector of non-negative elements, it results in another vector whose elements are non-negative. The main property of an M-type matrix is that its inverse contains non-negative elements and is therefore a positive operator [108]. 


\subsubsection{Boundary Conditions}

The boundary conditions need to be prescribed at the boundary of the computational domain. For the surface of an inviscid body or for a plane of symmetry, the flow has to remain tangent to the surface because no flow is allowed to cross the body surface. If we define the velocity vector as $\overrightarrow{\mathbf{V}}=u \overrightarrow{\mathbf{i}}+v \overrightarrow{\mathbf{j}}+w \overrightarrow{\mathbf{k}}$, we can express the boundary condition as:

$$
\overrightarrow{\mathbf{V}} \cdot \hat{\mathbf{n}}=0
$$

which can be expanded as:

$$
u \hat{n}_{x}+v \hat{n}_{y}+w \hat{n}_{z}=0
$$

where $\hat{\mathbf{n}}=\hat{n}_{x} \overrightarrow{\mathbf{i}}+\hat{n}_{y} \overrightarrow{\mathbf{j}}+\hat{n}_{z} \overrightarrow{\mathbf{k}}$. This is not enforced at the nodes but rather through the flux terms in the calculation of the residual. We have:

$$
\begin{aligned}
& \overrightarrow{\mathbf{F}}_{\mathbf{i}} \cdot \hat{\mathbf{n}}= \mathbf{f} \hat{n}_{x}+\mathbf{g} \hat{n}_{y}+\mathbf{h} \hat{n}_{z} \\
& \overrightarrow{\mathbf{F}}_{\mathbf{i}} \cdot \hat{\mathbf{n}}=\left\{\begin{array}{c}
\rho\left(u \hat{n}_{x}\right) \\
\rho u\left(u \hat{n}_{x}\right)+p \hat{n}_{x} \\
\rho v\left(u \hat{n}_{x}\right) \\
\rho w\left(u \hat{n}_{x}\right) \\
(E+p) u \hat{n}_{x}
\end{array}\right\}+\left\{\begin{array}{c}
\rho\left(v \hat{n}_{y}\right) \\
\rho u\left(v \hat{n}_{y}\right) \\
\rho v\left(v \hat{n}_{y}\right)+p \hat{n}_{y} \\
\rho w\left(v \hat{n}_{y}\right) \\
(E+p) v \hat{n}_{y}
\end{array}\right\}+\left\{\begin{array}{c}
\rho\left(w \hat{n}_{z}\right) \\
\rho u\left(w \hat{n}_{z}\right) \\
\rho v\left(w \hat{n}_{z}\right) \\
\rho w\left(w \hat{n}_{z}\right)+p \hat{n}_{z} \\
(E+p) w \hat{n}_{z}
\end{array}\right\}
\end{aligned}
$$

Using the boundary condition in Eq. 3.44, the expression above reduces to:

$$
\overrightarrow{\mathbf{F}}_{\mathbf{i}} \cdot \hat{\mathbf{n}}=\left\{\begin{array}{c}
0 \\
p \hat{n}_{x} \\
p \hat{n}_{y} \\
p \hat{n}_{z} \\
0
\end{array}\right\}
$$

For the case where the body is a viscous surface, the turbulence model requires that:

$$
\tilde{\nu}=0
$$

In addition, the no-slip condition is applied:

$$
\overrightarrow{\mathbf{V}}=0
$$

The wall temperature is prescribed as the adiabatic wall temperature:

$$
\frac{T_{\text {wall }}}{T_{\infty}}=1+\sqrt{\operatorname{Pr}} \frac{\gamma-1}{2} M_{\infty}^{2}
$$


We need to relate $T_{\text {wall }}$ to the internal energy $E$. In non-dimensional form, the temperature is related to the local speed of sound in a simple fashion:

$$
T=a^{2}=\frac{\gamma p}{\rho}
$$

The pressure can be expressed in terms of the internal energy $E$ :

$$
p=(\gamma-1)\left[E-\rho \frac{\left(u^{2}+v^{2}+w^{2}\right)}{2}\right]
$$

But since $\overrightarrow{\mathbf{V}}=0$ at the body surface, the expression for the pressure $p$ can be substituted in Eq. 3.50 to yield the prescribed internal energy at the wall:

$$
E=\frac{T_{\text {wall }}}{\gamma(\gamma-1)} \rho
$$

The treatment of the far-field boundaries requires a more detailed explanation. For onedimensional inviscid unsteady flow, there exists characteristic lines along which some flow properties are constant. Those characteristic lines are referred to as $C_{+}$and $C_{-}$. The slope of these characteristic lines in the $\mathrm{x}$ - $\mathrm{t}$ plane is:

$$
\frac{d x}{d t}=\left\{\begin{array}{l}
u+a \text { on } C_{+} \text {characteristic line } \\
u-a \text { on } C_{-} \text {characteristic line }
\end{array}\right.
$$

The Riemann invariants $R^{+}$and $R^{-}$are constant along the $C_{+}$and $C_{-}$characteristic lines respectively. For a calorically perfect gas undergoing an isentropic process, those quantities are expressed as:

$$
R^{+}=u+\frac{2 a}{\gamma-1}
$$

and

$$
R^{-}=u-\frac{2 a}{\gamma-1}
$$

If we know the value of $R^{+}$and $R^{-}$at a point, the local speed of sound $a$ and the velocity $u$ are obtained easily by solving simultaneously Eqs. 3.54 and 3.55:

$$
\begin{gathered}
a=\frac{\gamma-1}{4}\left(R^{+}-R^{-}\right) \\
u=\frac{1}{2}\left(R^{+}+R^{-}\right)
\end{gathered}
$$


For two- and three-dimensional CFD calculations, the data is reconstructed at the far-field boundaries using the Riemann invariants by assuming that the far-field is locally 1-D. The velocity components normal to the boundary are used to evaluate $R^{+}$and $R^{-}$. For convenience, the normal component of the local velocity is defined as:

$$
\bar{U} \equiv \overrightarrow{\mathbf{V}} \cdot \hat{\mathbf{n}}
$$

Similarly, the component of the freestream velocity normal to the boundary is:

$$
\bar{U}_{\infty} \equiv \overrightarrow{\mathbf{V}}_{\infty} \cdot \hat{\mathbf{n}}
$$

Let us consider the case where the flow is subsonic with $-a \leq \bar{U} \leq a$. Since $\bar{U}+a \geq 0, R^{+}$ is evaluated from the data stored at the node on the outer boundary:

$$
R^{+}=\bar{U}+\frac{2 a}{\gamma-1}
$$

$R^{-}$is calculated based on the freestream quantities because $\bar{U}-a \leq 0$ :

$$
R^{-}=\bar{U}_{\infty}-\frac{2 a_{\infty}}{\gamma-1}
$$

For supersonic flow where $\bar{U}>a, R^{+}$is calculated from Eq. 3.60. However, $R^{-}$is computed from the interior values as well because $\bar{U}-a>0$ :

$$
R^{-}=\bar{U}-\frac{2 a}{\gamma-1}
$$

Similarly, for supersonic flow where $\bar{U}<-a, R^{-}$is evaluated from Eq. 3.61. In turn, $R^{+}$is based on the freestream quantities because $\bar{U}+a<0$ :

$$
R^{+}=\bar{U}_{\infty}+\frac{2 a_{\infty}}{\gamma-1}
$$

Once the Riemann invariants are determined, it is easy to obtain the local speed of sound and the velocity at the boundary from Eqs. 3.56 and 3.57:

$$
\begin{gathered}
a_{\text {boundary }}=\frac{\gamma-1}{4}\left(R^{+}-R^{-}\right) \\
\bar{U}_{\text {boundary }}=\frac{1}{2}\left(R^{+}+R^{-}\right)
\end{gathered}
$$

If $\bar{U}_{\text {boundary }} \geq 0$, the flow leaves the computational domain. The velocity on the boundary $\overrightarrow{\mathbf{V}}_{\text {boundary }}$ is extrapolated from the interior data:

$$
\overrightarrow{\mathbf{V}}_{\text {boundary }}=\overrightarrow{\mathbf{V}}+\left(\bar{U}_{\text {boundary }}-\bar{U}\right) \hat{\mathbf{n}}
$$


On the other hand, if we have an inflow boundary where $\bar{U}_{\text {boundary }}<0$, the velocity at the outer boundary is extrapolated from outside:

$$
\overrightarrow{\mathbf{V}}_{\text {boundary }}=\overrightarrow{\mathbf{V}}_{\infty}+\left(\bar{U}_{\text {boundary }}-\bar{U}_{\infty}\right) \hat{\mathbf{n}}
$$

The entropy $S_{\text {boundary }}$ is then calculated from freestream conditions if it is an inflow boundary and from the local nodal values otherwise. The density $\rho_{\text {boundary }}$ is obtained from the relationship for an isentropic process:

$$
\rho_{\text {boundary }}=\left(\frac{a_{\text {boundary }}^{2}}{\gamma S_{\text {boundary }}}\right)^{\frac{1}{\gamma-1}}
$$

The flux terms are evaluated based on those quantities and integrated along the outer boundary. The far-field boundary condition is enforced through the flux terms, not by imposing freestream conditions at the nodes on the boundary.

The treatment of the far-field boundaries is performed in a similar fashion for the turbulence equation. The dependent variable $\tilde{\nu}$ is extrapolated from the interior for outflow and is set to freestream otherwise.

\subsubsection{Time Integration}

The flow solver integrates the solution in time using the backward-Euler time-stepping scheme. At each iteration a linear system of equations needs to be solved. If we take Eq. 3.31:

$$
V \frac{\Delta \mathbf{Q}}{\Delta t}+\sum_{S}\left(\overrightarrow{\mathbf{F}}_{\mathbf{i}} \cdot \hat{\mathbf{n}}\right) \Delta S-\sum_{S}\left(\overrightarrow{\mathbf{F}}_{\mathbf{v}} \cdot \hat{\mathbf{n}}\right) \Delta S=0
$$

And if we use the definition of the residual defined in Eq. 3.32:

$$
\mathbf{R}=\sum_{S}\left(\overrightarrow{\mathbf{F}}_{\mathbf{i}} \cdot \hat{\mathbf{n}}\right) \Delta S-\sum_{S}\left(\overrightarrow{\mathbf{F}}_{\mathbf{v}} \cdot \hat{\mathbf{n}}\right) \Delta S
$$

We can write:

$$
V \frac{\Delta Q}{\Delta t}=-\mathbf{R}
$$

For a given time step $n$, the implicit Euler scheme takes the form:

$$
\left(\frac{V}{\Delta t}+\frac{\partial \mathbf{R}^{n}}{\partial \mathbf{Q}}\right) \Delta \mathbf{Q}^{n}=-\mathbf{R}^{n}
$$


where

$$
\Delta \mathbf{Q}^{n}=\mathrm{Q}^{n+1}-\mathrm{Q}^{n}
$$

This implicit scheme has the advantage of being stable even for large time steps as opposed to a simple explicit scheme. The right-hand side of Eq. 3.70 is evaluated at each time step by adding the contributions of the inviscid and viscous flux terms to the residual. This procedure was detailed in section 3.2.3. The Jacobian $\frac{\partial \mathbf{R}}{\partial \mathbf{Q}}^{n}$ is evaluated by differentiating the residual $\mathbf{R}$ and the flux terms with respect to the vector of dependent variables $\mathbf{Q}$. A first-order discretization is used.

The system of equations is solved for $\Delta \mathbf{Q}^{n}$ using the Jacobi iterative scheme and the nodal values of $\mathbf{Q}$ are updated at each step:

$$
\mathbf{Q}^{n+1}=\mathbf{Q}^{n}+\Delta \mathbf{Q}^{n}
$$

To integrate the solution quickly to steady state, large time steps $\Delta t$ are necessary. However, in order for the system of equations to be solved properly at each iteration, it is important that it is diagonally dominant. That means that large time steps can have a detrimental effect on the convergence of the Jacobi iteration. The flow solver uses local time stepping and the time step is determined from the Courant-Friedrich-Levy (CFL) number through the following expression:

$$
\Delta t=\mathrm{CFL} \frac{V}{\int_{\partial \Omega}(|\overrightarrow{\mathbf{V}} \cdot \hat{\mathbf{n}}|+a) d S}
$$

where $V$ is the volume of the control volume and $\overrightarrow{\mathbf{V}} \cdot \hat{\mathbf{n}}$ represents the velocity component normal to the control volume surface.

\subsubsection{Multigrid}

For the two-dimensional calculations, the flow solver allows for the use of multigrid acceleration [105]. This method solves the equations on a series of grids with various coarsening levels. It interpolates the solution from the fine grid to coarser ones in order to get rid of the low-frequency numerical errors. The solution is projected back to the finer grids to improve the accuracy. This results in an improved convergence rate of the solution. More details about multigrid can be obtained from Briggs [109]. This feature is not available in the three-dimensional version of the flow solver. 


\section{Chapter 4}

\section{Aerodynamic Analysis of a Strut-Braced Wing Aircraft Using the Euler Equations}

The strut-braced wing concept has been introduced to provide a significant improvement over conventional cantilever designs in the transonic flow regime. The concept adds synergism between aerodynamics, structures, and propulsion in order to provide a lighter airplane for commercial transport. The MDO studies performed at Virginia Tech $[1,2,3]$ have produced several derivatives of the strut-braced wing aircraft for cruise at Mach 0.85. The purpose of this chapter is to provide an insight in the application of CFD capability to one of the configurations obtained after optimization. For this initial study, an inviscid analysis using the Euler equations was employed.

In this chapter, the CFD tools used for the flow analysis are presented and validated. A description of the strut-braced wing concept to be studied will follow. Results obtained for a wing alone, a wing with a straight strut, and a wing with an arch-shaped strut are discussed. An equation representing the interference drag penalty in terms of the arch radius is established.

\subsection{Approach}

This aerodynamic study of the strut-braced wing concept was performed on inviscid unstructured grids generated with the program VGRIDns [100]. This code implements an 
advancing-front algorithm to generate the tetrahedra in the computational space. To reduce the memory required to perform the flow solutions, grid stretching can be employed along directions of small gradients. A detailed discussion of the grid generation process was provided in Chapter 3.

The flow solver utilized to solve the Euler equations was USM3D [95, 110]. The latest version of the code employed in this work can be run on multiple processors. USM3D uses a tetrahedral, cell-centered, finite-volume formulation. The inviscid fluxes are evaluated using the flux-difference splitting scheme of Roe [107]. The data reconstruction is second-order accurate in space. The solution is marched in time using a linearized, backward Euler time differencing technique.

\subsection{Validation}

In order to assess the accuracy of the solution obtained with the flow solver, the case of the ONERA M6 wing [111] in transonic flow was studied. The wing has a round wing tip with a root chord of $0.8059 \mathrm{~m}$, a half-span of $1.1963 \mathrm{~m}$, and a leading edge sweep of 30 degrees. The wing has a taper ratio equal to 0.562 . The freestream conditions are such that $M_{\infty}=0.84$ with an angle of attack $\alpha=3.06^{\circ}$.

The computational box extends 10 root chords to the far-field boundary. The unstructured grid generated makes use of moderate stretching along the span. A total of 12,662 triangles were generated on the wing and on the six faces of the computational box. The total number of tetrahedra in the mesh is equal to 425,307 . The resulting surface grid is pictured in Figure 4.1.

The CFD solution was obtained after the residual was reduced by three orders of magnitude, corresponding to the convergence of the drag coefficient. The Mach number contours are shown in Figure 4.2. The lambda-shock on the wing surface seems to be resolved accurately.

The pressure distribution is compared with the experimental data in Figure 4.3. In general, the agreement with the experiment is good except for the shock resolution on the wing upper surface. For the $\eta=80 \%$ station for instance, the computation misses the double shock. The reason for that is that the grid is not adequate in the region where the shocks take place. The viscous effects are not considered in the numerical solution, which 


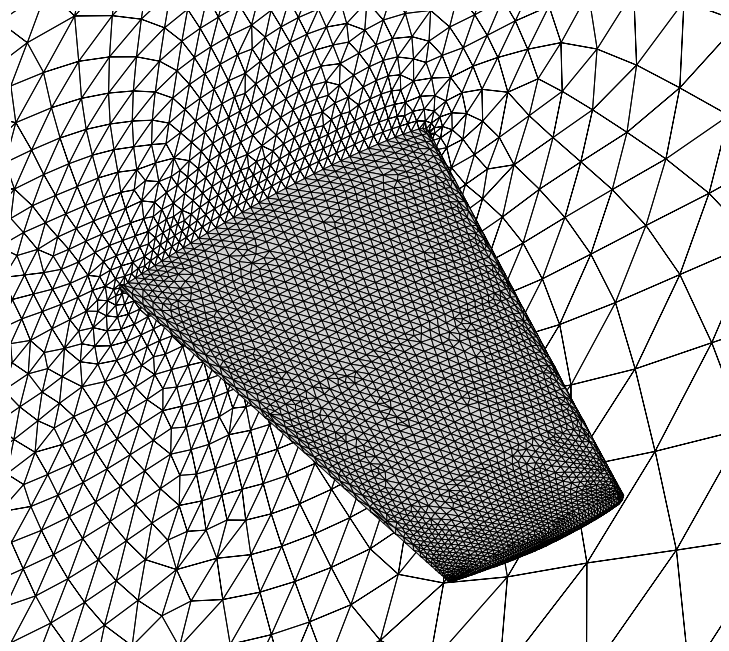

(a)

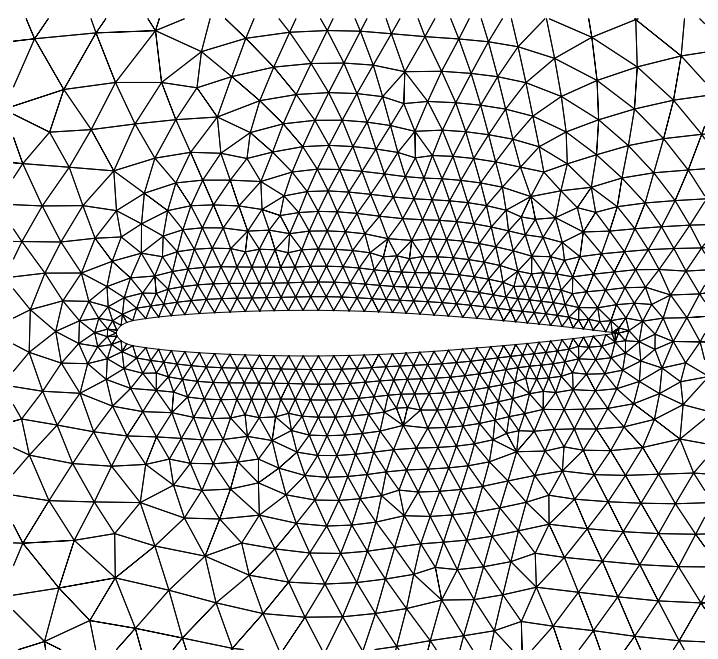

(b)

Figure 4.1: Surface mesh on the ONERA M6 wing (a) Wing surface and symmetry plane (b) Triangulation of the symmetry plane
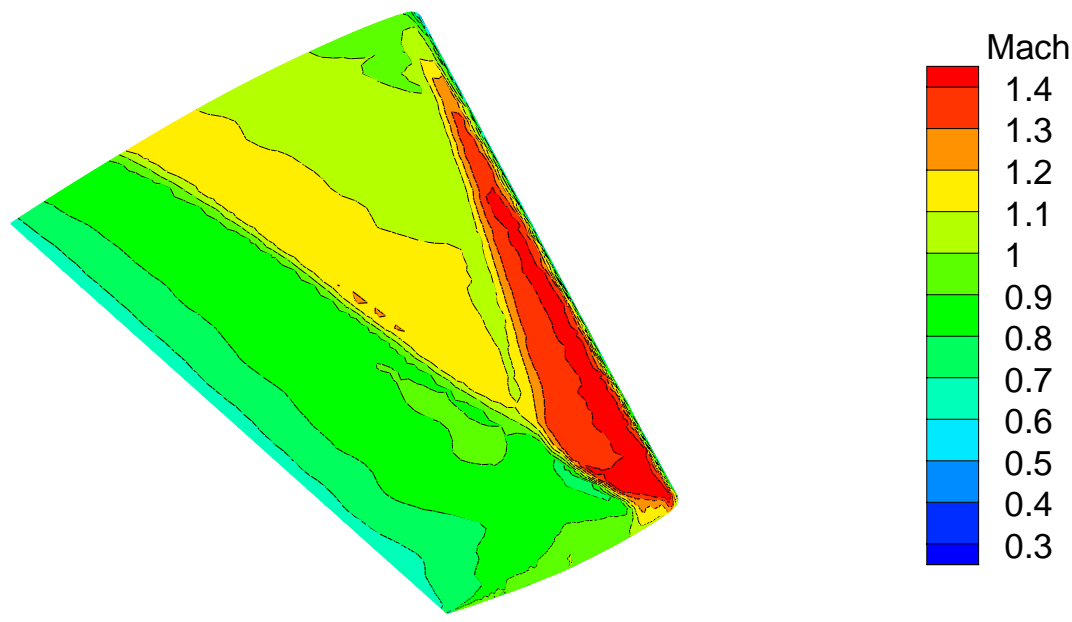

Figure 4.2: Mach number contours on the ONERA M6 wing surface 
justifies the poor resolution of the separation region at $\eta=99 \%$. The calculation matches the data very well for all stations on the wing lower surface. The Euler solution captures the principal flow features, and is therefore considered appropriate for preliminary design applications.

\subsection{Description of the Airplane}

The strut-braced wing concept presented in this section was obtained at an early stage of development of the MDO tools. Therefore, it would be misleading to compare the configuration directly with the most recent results $[2,3]$ because more refined analyses are employed in the latest version of the MDO code. However, it should give a flavor of some of the features of the strut-braced wing concept.

The concept referred to as SS5 was obtained after performing an optimization to minimize the take-off gross weight while meeting all the aerodynamic and structural constraints. The airplane is designed to carry 325 passengers and fly at a cruise Mach number of 0.85 to cover a range of 7,500 nautical miles with a 500-nautical mile reserve. The wing is fixed on top of the fuselage. The configuration is equipped with engines mounted at the wing tip to provide some aerodynamic benefits because the tip vortex is swallowed inside the engine. To simplify the CFD analysis of the configuration, the engines are left off.

The planform geometry is detailed in Table 4.1. The wing span is relatively large compared to conventional cantilever designs. There are two different taper ratios for the inboard and outboard sections of the wing. The strut intersects the wing near the tip, at the point where the inboard and outboard sections of the main wing meet. The strut is tapered and its chord increases from the root to the tip. The sweep of the strut is less than the sweep of the main wing. The main wing was designed with a supercritical airfoil section, namely $\mathrm{SC}(2)-0706$ [112]. It has a $t / c$ ratio of $6 \%$. The strut airfoil section is a $3.5 \%$-thick section of the NACA 65A-family, the NACA 65A003.5 airfoil. These airfoil sections were chosen by considering the geometric data provided by the MDO process.

In order to obtain the wing twist distribution, the program LAMDES $[113,114]$ was employed. Given the geometry of the wing planform and the design lift coefficient, LAMDES calculates the spanload distribution that provides the minimum induced drag. It also computes the rigid twist distribution assuming linear aerodynamics. The computed twist distribution 


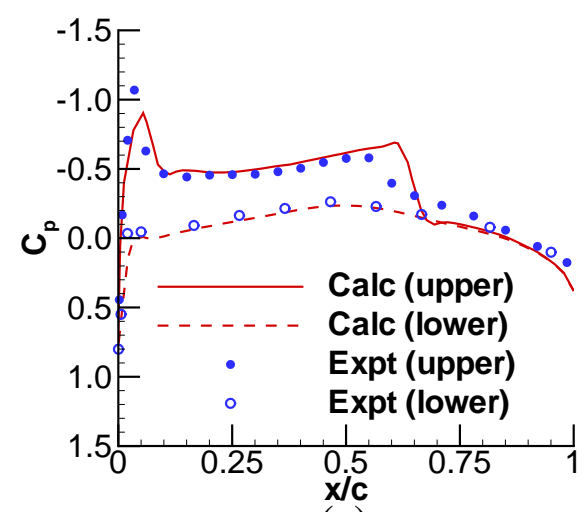

(a)

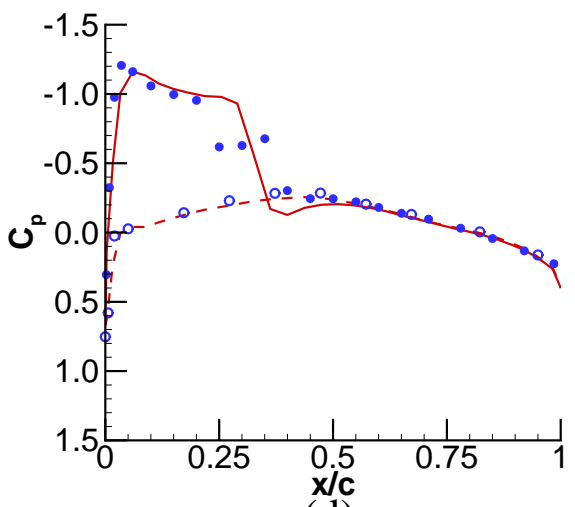

(d)

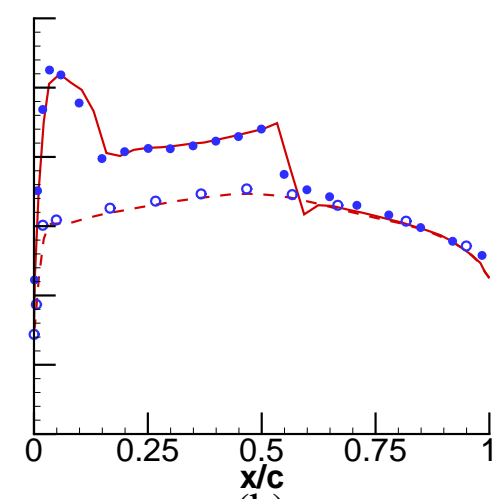

(b)

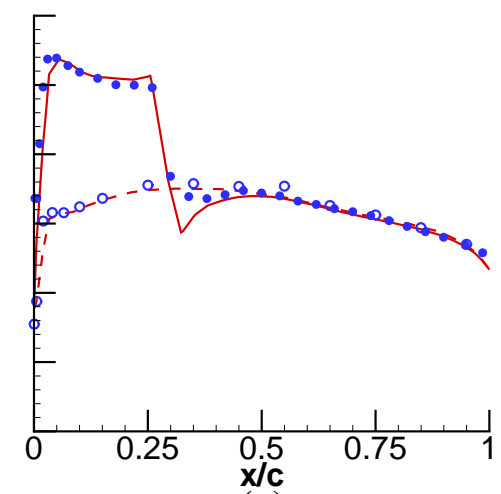

(e)

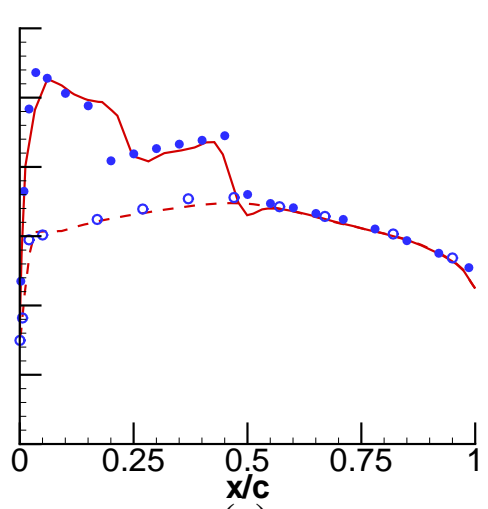

(c)

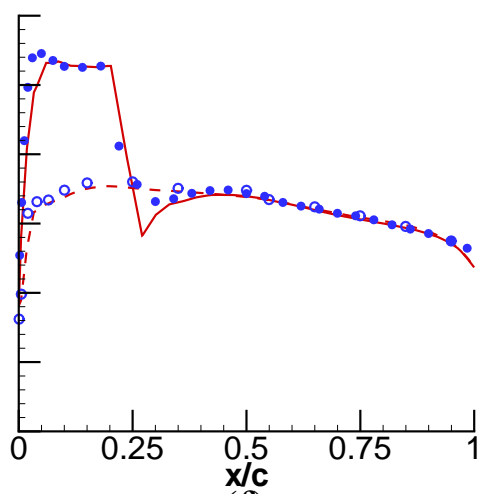

(f)

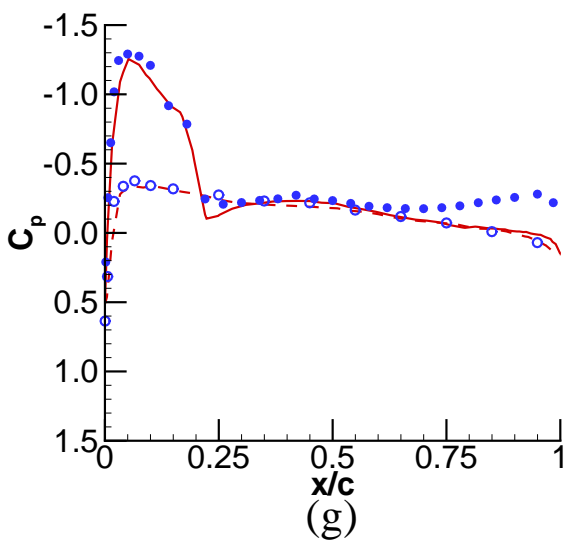

Figure 4.3: Pressure distribution on the ONERA M6 wing (a) $\eta=20 \%$ (b) $\eta=44 \%$ (c) $\eta=65 \%$ (d) $\eta=80 \%$ (e) $\eta=90 \%$ (f) $\eta=95 \%$ (g) $\eta=99 \%$ 
Table 4.1: Geometry of the strut-braced wing concept SS5

\begin{tabular}{|l|c|}
\hline Wing half-span (ft) & 99.5 \\
\hline$\frac{1}{4}$-chord wing sweep (deg) & 27.3 \\
\hline Wing root chord (ft) & 27.1 \\
\hline Wing mid chord (ft) & 13.7 \\
\hline Wing tip chord (ft) & 8.7 \\
\hline Fuselage height (ft) & 20.3 \\
\hline $\begin{array}{l}\text { Spanwise position of wing-strut } \\
\text { intersection, (\% of half-span) }\end{array}$ & 87.5 \\
\hline $\begin{array}{l}\text { Spanwise position of chord } \\
\text { breakpoint, (\% of half-span) }\end{array}$ & 87.5 \\
\hline$\frac{1}{4}$-chord strut sweep (deg) & 17.7 \\
\hline Strut root chord (ft) & 4.5 \\
\hline Strut tip chord (ft) & 5.8 \\
\hline Reference area (ft ${ }^{2}$ ) & 3828.7 \\
\hline Cruise lift coefficient & 0.5191 \\
\hline
\end{tabular}

is depicted in Figure 4.4. It is imposed on the wing by rotating the airfoil about its leading edge. More considerations are usually involved in determining the location of the axis of rotation. The location of the flap devices is one constraint that usually needs to be taken into account. The airfoil section chosen for the main wing is aligned with the streamwise direction.

\subsection{Analysis of the Wing Alone}

To begin the study of the aerodynamics of the strut-braced wing configuration, the wing alone was first analyzed. A symmetry plane is employed at the wing root and the farfield boundary is located 10 root chords away from the wing. An inviscid unstructured grid containing 24,276 surface triangles and 941,932 tetrahedral cells was generated with moderate stretching along the span. The surface mesh on the wing upper and lower surfaces is depicted in Figure 4.5.

The flow solver USM3D was employed to calculate the transonic flow over the wing. The freestream Mach number chosen corresponds to the cruise Mach number of the airplane, namely $M_{\infty}=0.85$. Since the incidence angle of the wing is not known a priori, the flow 


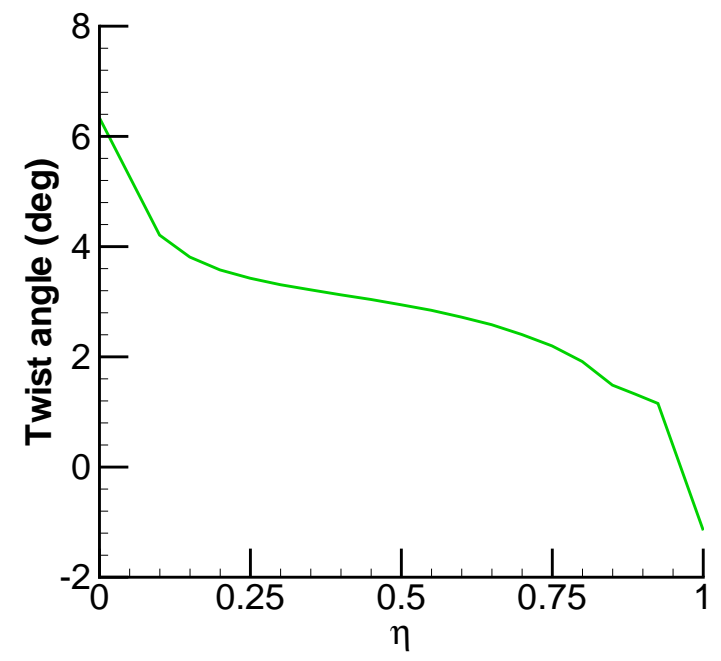

Figure 4.4: Wing twist distribution for the strut-braced wing $\left(C_{L}=0.5191\right)$

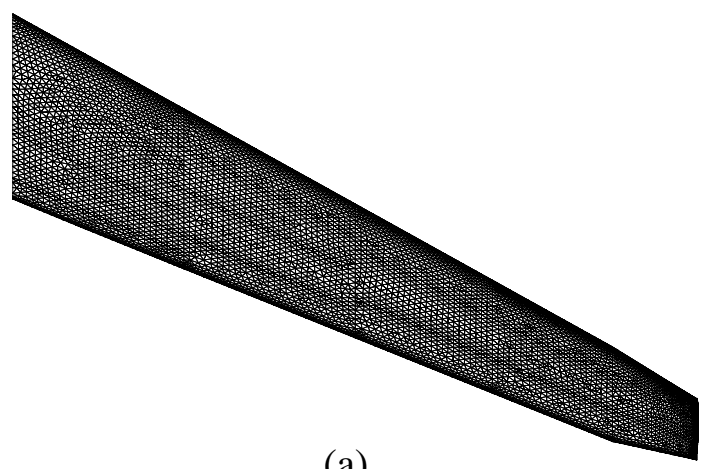

(a)

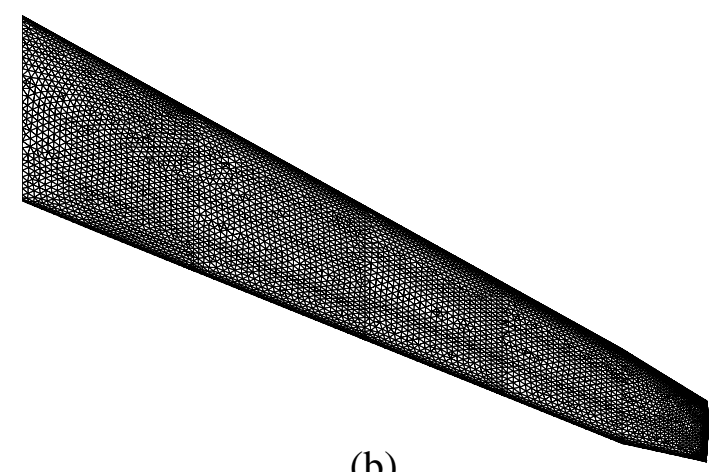

(b)

Figure 4.5: Surface grid on the SS5 wing (a) Wing upper surface (b) Wing lower surface 


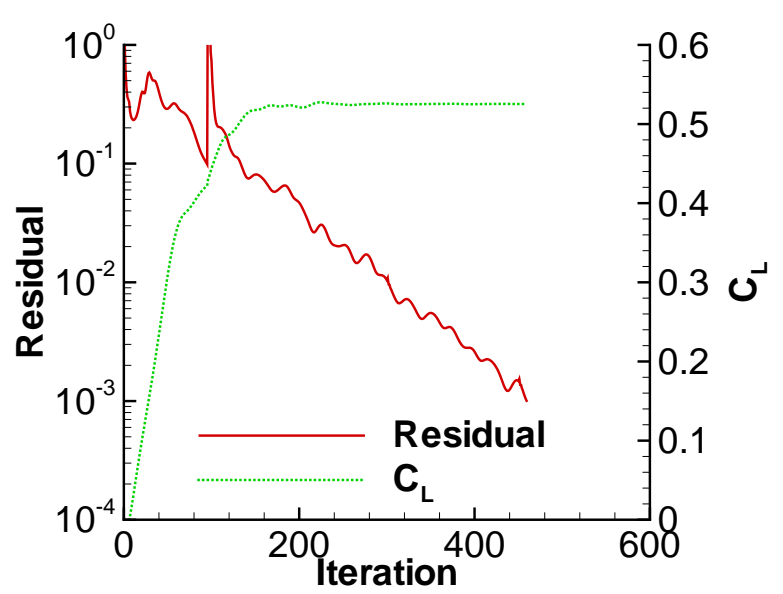

(a)

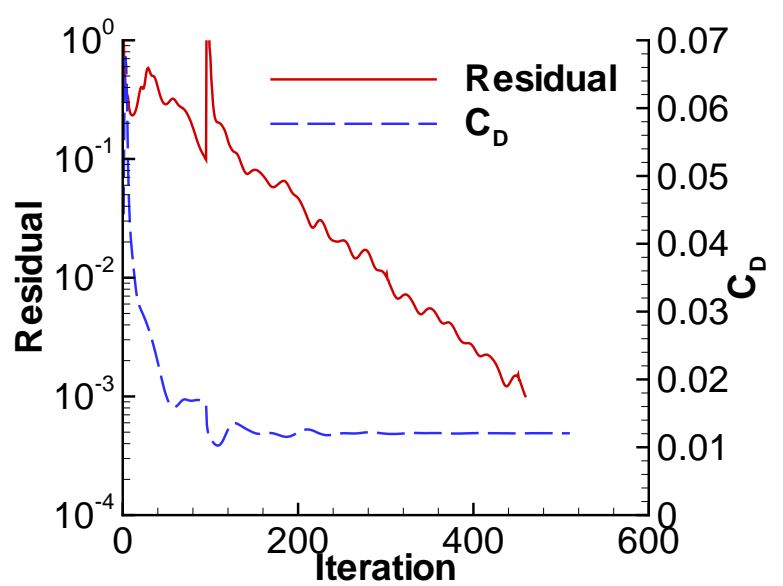

(b)

Figure 4.6: Convergence history, $M_{\infty}=0.85, \alpha_{\text {root }}=2.36^{\circ}\left(\alpha=-3.97^{\circ}\right)$ (a) Lift coefficient and residual (b) Drag coefficient and residual

solver determines iteratively the angle of attack that will provide the required lift coefficient. A cruise lift coefficient $C_{L}$ of 0.5253 was achieved using an angle of attack $\alpha=-3.97^{\circ}$. Since the wing was pre-twisted based on the results of LAMDES, this angle of attack is equivalent to an incidence $\alpha_{\text {root }}=2.36^{\circ}$ at the wing root. The drag coefficient calculated by USM3D for the wing is equal to 0.0121 .

The flow solution was converged three orders of magnitude. The convergence history is pictured in Figure 4.6. The flow solver is run with first order spatial discretization the first 95 iterations. The sudden change in residual after that point corresponds to a change in discretization to second order in space. When the residual has been decreased three orders of magnitude, there is no change in the lift and drag coefficients. This convergence criterion is applied to all the calculations presented in this chapter.

In order to determine if the grid resolution was sufficient on the wing surface, a grid convergence study was conducted. In addition to the fine grid dicussed already, a medium, a coarse, and a very coarse grids were generated. The surface mesh on the wing upper surface is shown for all grids in Figure 4.7. The characteristics of the grids are listed in Table 4.2. The flow solutions obtained on the medium and coarse grids produced lift and drag coefficients that are comparable with the results on the fine grid. The lift coefficient depends less upon the mesh size than the drag coefficient. The data presented in Table 4.2 is depicted in terms of the inverse of the number of cells in Figure 4.8. Both curves seem 
Table 4.2: Grid convergence study for the wing alone, $M_{\infty}=0.85, \alpha_{\text {root }}=2.36^{\circ}\left(\alpha=-3.97^{\circ}\right)$

\begin{tabular}{|l|c|c|c|c|}
\hline Grid & $\begin{array}{c}\text { Number of surface } \\
\text { triangles }\end{array}$ & $\begin{array}{c}\text { Total number } \\
\text { of cells }\end{array}$ & $C_{L}$ & $C_{D}$ \\
\hline \hline Very coarse & 1,948 & 29,061 & 0.4867 & 0.0144 \\
Coarse & 6,660 & 147,537 & 0.5187 & 0.0133 \\
Medium & 11,490 & 317,601 & 0.5229 & 0.0126 \\
Fine & 24,276 & 941,932 & 0.5253 & 0.0121 \\
\hline
\end{tabular}

to be reaching as asymptotic value for the lift and drag coefficients on the fine grid. That grid was judged satisfactory for the purpose of this work and its grid point distribution was employed for the cases presented later in this chapter.

The principle of Richardson extrapolation [115] is applied to determine the error in the lift and drag coefficients on the finest mesh. Theoretically, that method should be used for sets of grids that are refined in a consistent fashion. Such is not the case here because the unstructured grid methodology used doesn't provide an easy way to achieve that. However, the results will give an estimate of the magnitude of the error on the fine mesh. Taking the grid refinement ratio $r$ as the ratio of the total number of nodes on the fine grid to the number of nodes on the medium grid, the exact value of the lift and drag coefficients $C_{L_{\text {exact }}}$ and $C_{D_{\text {exact }}}$ can be approximated as:

$$
\begin{gathered}
C_{L_{\text {exact }}} \approx C_{L_{\text {fine }}}+\frac{C_{L_{\text {fine }}}-C_{L_{\text {medium }}}}{r^{2}-1} \\
C_{D_{\text {exact }}} \approx C_{D_{\text {fine }}}+\frac{C_{D_{\text {fine }}}-C_{D_{\text {medium }}}}{r^{2}-1}
\end{gathered}
$$

From these equations, $C_{L_{\text {exact }}}=0.5256$ and $C_{D_{\text {exact }}}=0.0120$ are computed. On the fine mesh, the error is small for both cases: 0.0003 for the lift coefficient and $6 \times 10^{-5}$ for the drag coefficient.

For the fine grid, the Mach number contours on the wing surface are pictured in Figure 4.9. The contour lines coalesce near the trailing edge where the shock forms. The spanload distribution obtained by integrating the pressure on the wing surface at several span stations is shown in Figure 4.10. The load follows an elliptic distribution with some small variations. The variations are most likely due to the fact that the load is computed at discrete locations along the wing span. 


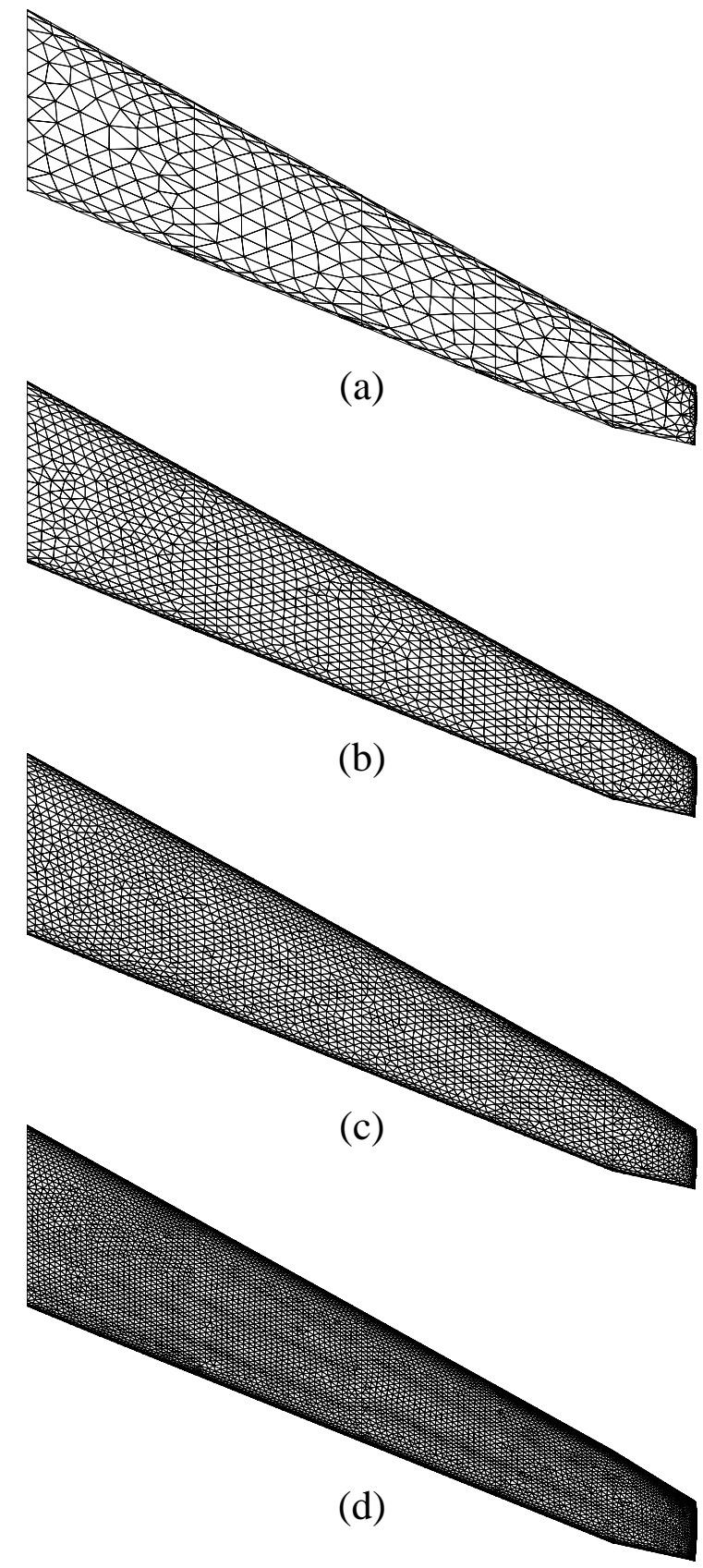

Figure 4.7: Mesh on the wing upper surface (a) Very coarse grid (b) Coarse grid (c) Medium grid (d) Fine grid 


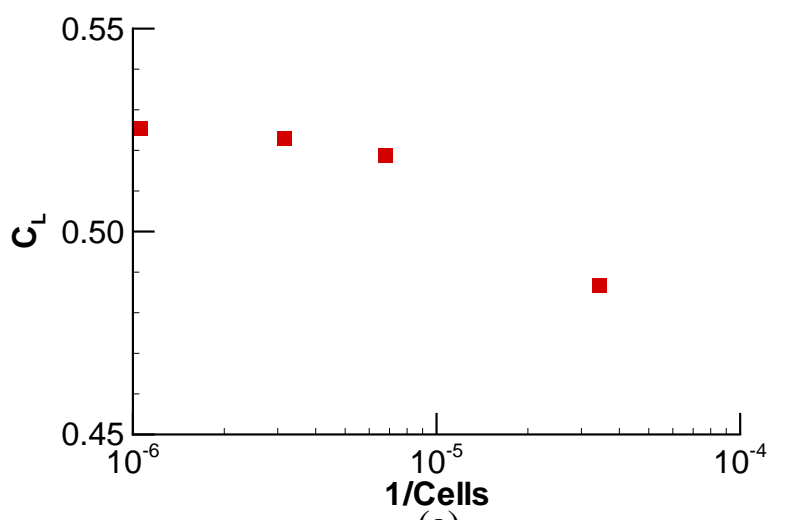

(a)

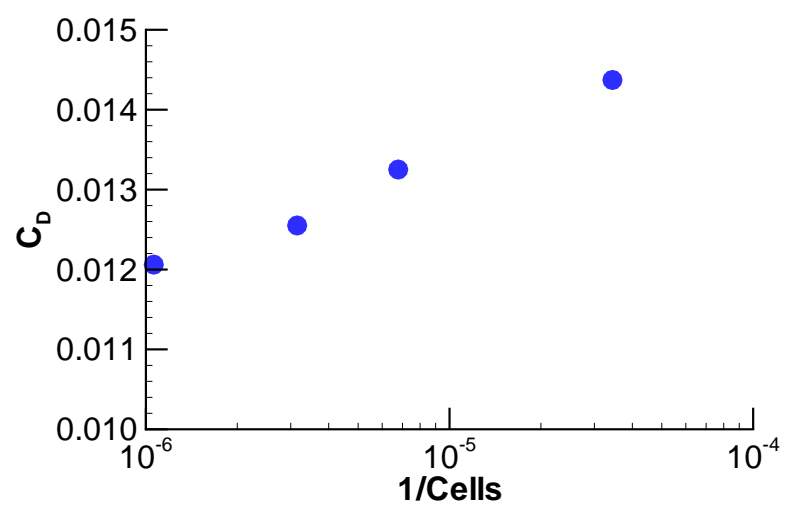

(b)

Figure 4.8: Effect of the grid refinement on the lift and drag coefficients for the wing alone, $M_{\infty}=0.85, \alpha_{\text {root }}=2.36^{\circ}\left(\alpha=-3.97^{\circ}\right)$ (a) Variation of the lift coefficient (b) Variation of the drag coefficient

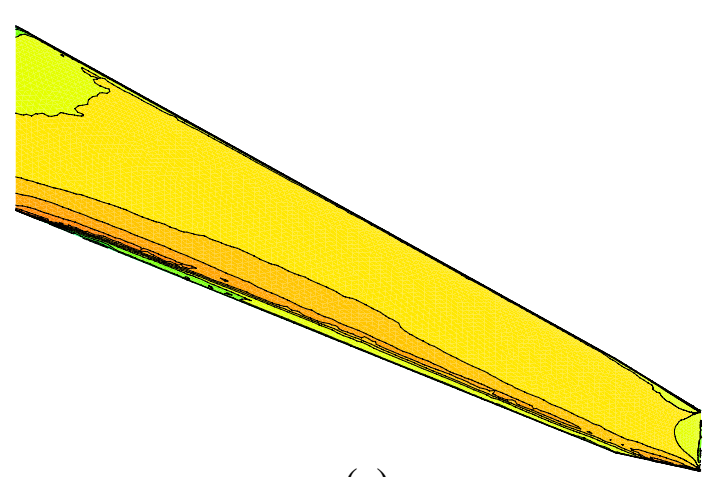

(a)
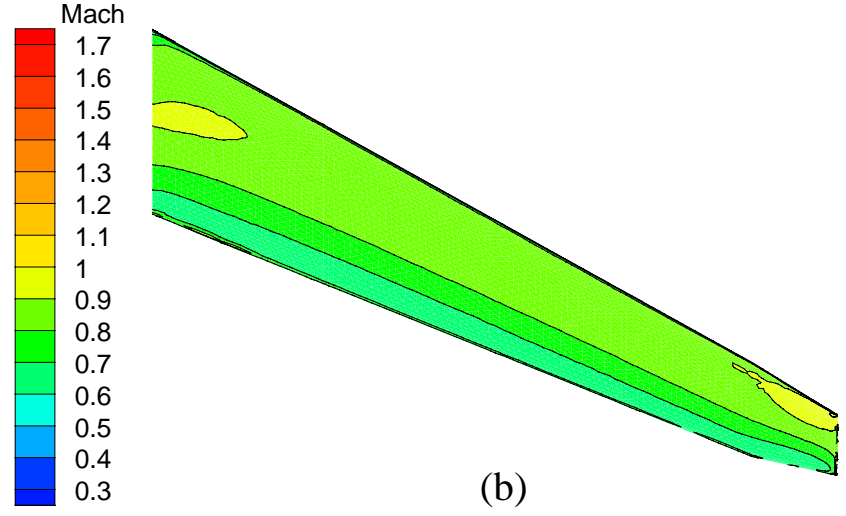

(b)

Figure 4.9: Mach number contours on the SS5 clean wing, $M_{\infty}=0.85, \alpha_{\text {root }}=2.36^{\circ}$ $\left(\alpha=-3.97^{\circ}\right)$ (a) Wing upper surface (b) Wing lower surface 


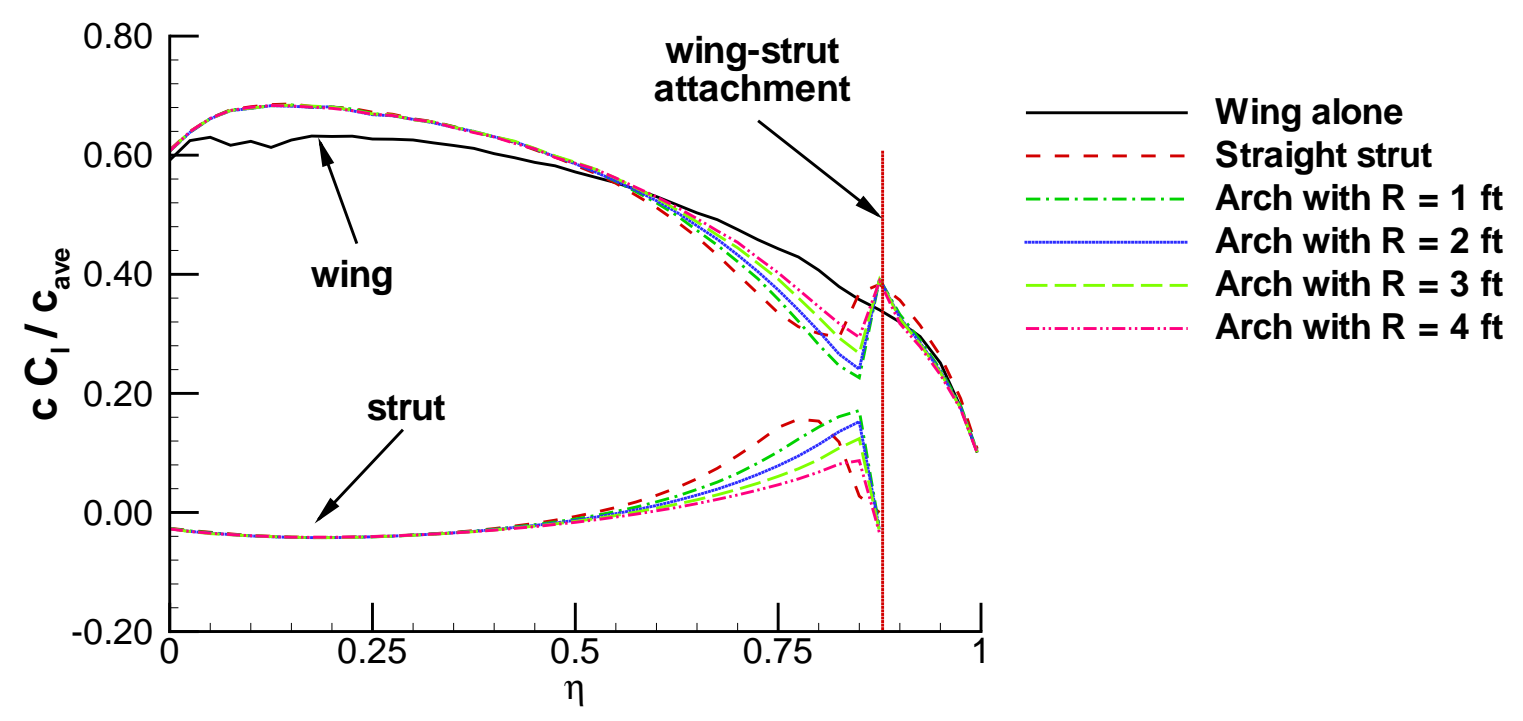

Figure 4.10: Influence of the strut on the spanload distribution of the SS5 strut-braced wing design, $M_{\infty}=0.85, \alpha_{\text {root }}=2.36^{\circ}\left(\alpha=-3.97^{\circ}\right)$

The pressure distributions at several stations along the wing span are shown in Figure 4.16 for all grids. It can be noticed that the pressure on the upper surface is very flat except at the wing root. A shock is located between $x / c=0.85$ and 0.95 along the chord. The sudden variation in pressure in the vicinity of the trailing edge can be explained by the slight mismatch between the design Mach number and the design lift coefficient of the airfoil. The wing sections would require some modifications to achieve a better design.

\subsection{Analysis of the Strut-Braced Wing}

The details of the strut-braced wing configuration provided in Table 4.1 do not specify the shape of the strut to be employed. Two alternatives have been studied in this project: a straight strut or a strut shaped like an arch in the region where it intersects the wing. The straight strut offers the advantage of simplicity, but is accompanied by a substantial drag penalty near the junction because of the sharp angle made by the strut and the wing. The constrained flow channel formed near the junction causes disruptions in the flow on the wing surface. The arch-shaped strut is designed to curve near its tip in order to intersect the wing surface perpendicularly. That way, the angle made by the wing and the strut is optimum 


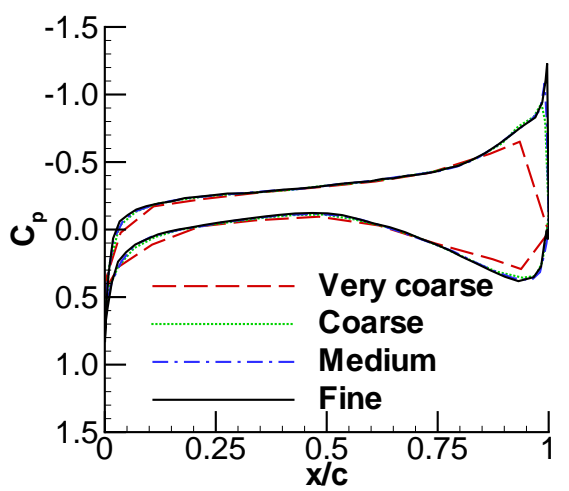

(a)

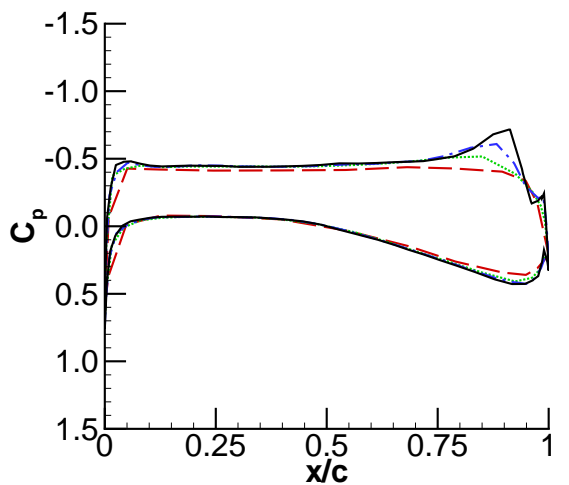

(d)

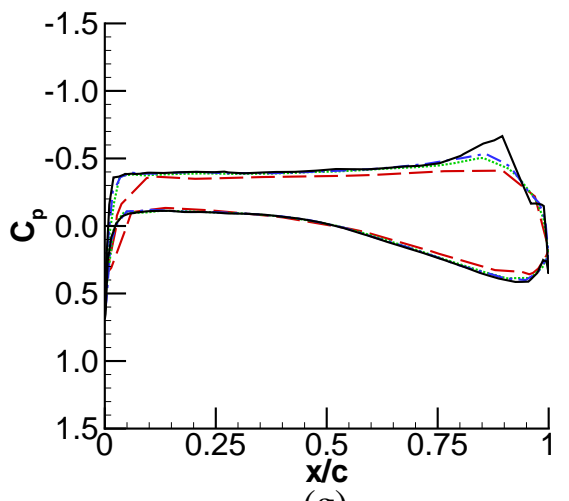

$(\mathrm{g})$

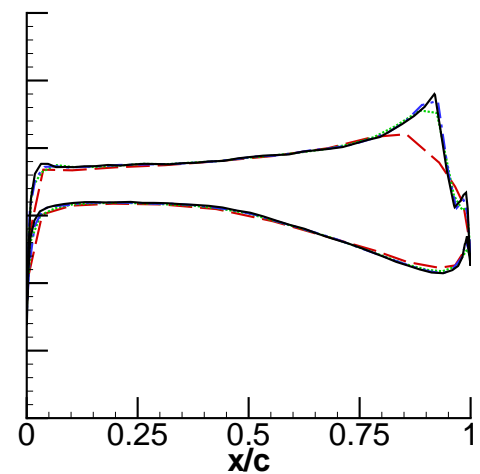

(b)

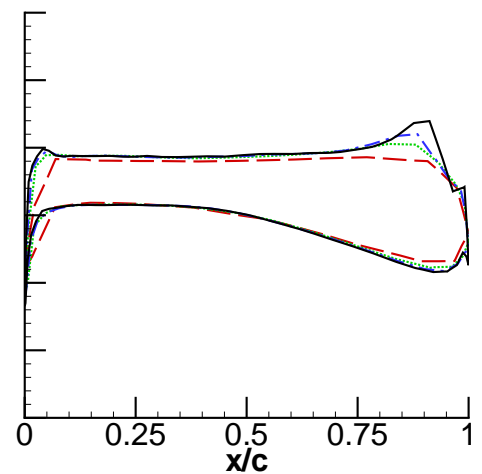

(e)

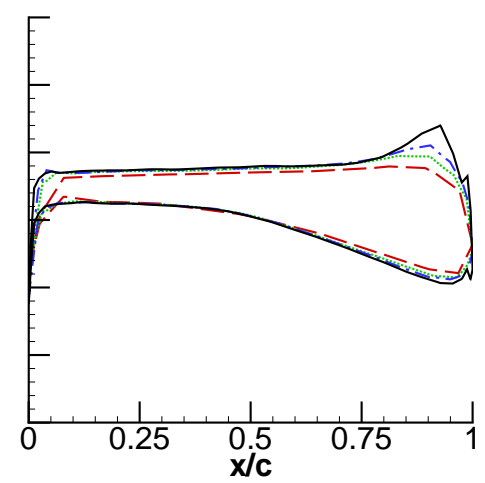

(h)

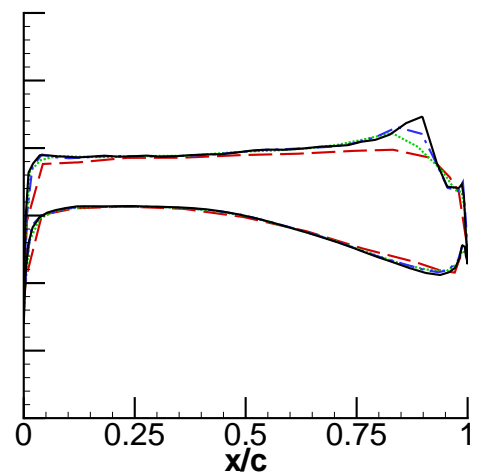

(c)

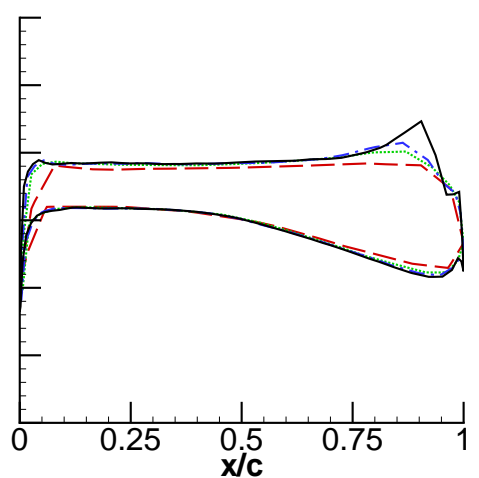

(f)

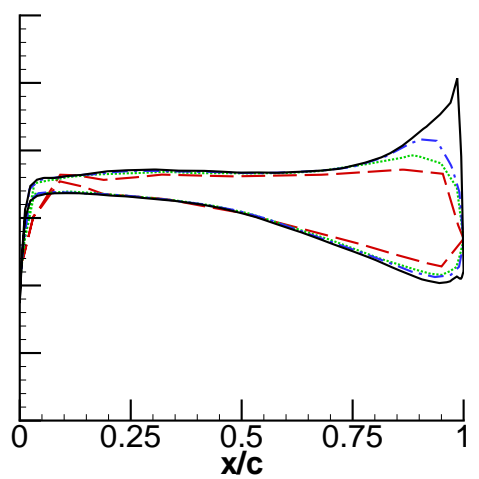

(i)

Figure 4.11: Effect of the grid refinement on the pressure distribution for the wing alone, $M_{\infty}=0.85, \alpha_{\text {root }}=2.36^{\circ}\left(\alpha=-3.97^{\circ}\right)$ (a) $\eta=0 \%$ (b) $\eta=25 \%$ (c) $\eta=50 \%$ (d) $\eta=70 \%$ (e) $\eta=75 \%$ (f) $\eta=80 \%$ (g) $\eta=85 \%$ (h) $\eta=90 \%$ (i) $\eta=95 \%$ 


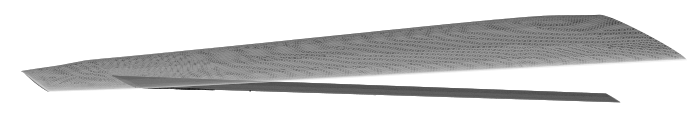

(a)

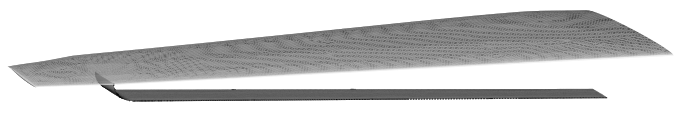

(b)

Figure 4.12: Strut-braced wing designs (a) With straight strut (b) With arch-shaped strut

and the increased distance between them alleviates the flowfield disruption in the vicinity of the junction. Strut-braced wing designs employing a straight strut and an arch-shaped strut are depicted in Figure 4.12.

To evaluate and compare the various possibilities for the shape of the strut, configurations employing a straight strut and an arch-shaped strut with a radius of $1,2,3$, and 4 ft were studied. For structural considerations, loading the strut should be avoided as much as possible. Hence, a twist distribution should be employed on the strut to obtain zero load everywhere along its length. As a first cut to obtain zero loading, the strut was rotated about its leading edge with the same twist angle everywhere. That rotation angle was taken to be $-3.97^{\circ}$ since this is the angle of attack of the freestream determined in Section 4.4.

The configuration is enclosed by a computational box extending 10 wing root chords to the far-field boundaries from the main wing. A symmetry boundary condition was used at the plane where the wing and the strut connect with the fuselage. The grid point distribution used on the main wing is based on the fine mesh generated for the grid convergence study presented in Figure 4.8. For all strut arrangements, similar inviscid grids employing moderate stretching along the wing and strut spans were generated. The surface grid for the strutbraced wing design with a straight strut is portrayed in Figure 4.13. The grid characteristics are detailed in Table 4.3 for each configuration as well as the clean wing case discussed in Section 4.4 .

The freestream conditions were set to the values employed for the clean wing: $M_{\infty}=0.85$, $\alpha_{\text {root }}=2.36^{\circ}\left(\alpha=-3.97^{\circ}\right)$. The Mach number contours for the strut-braced wing with a straight strut are shown in Figure 4.14 and for an arch-shaped strut with a 4-ft radius in Figure 4.15. The upper wing surface of both configurations doesn't show any disruption due to the presence of the strut underneath. One can hardly distinguish them from the 


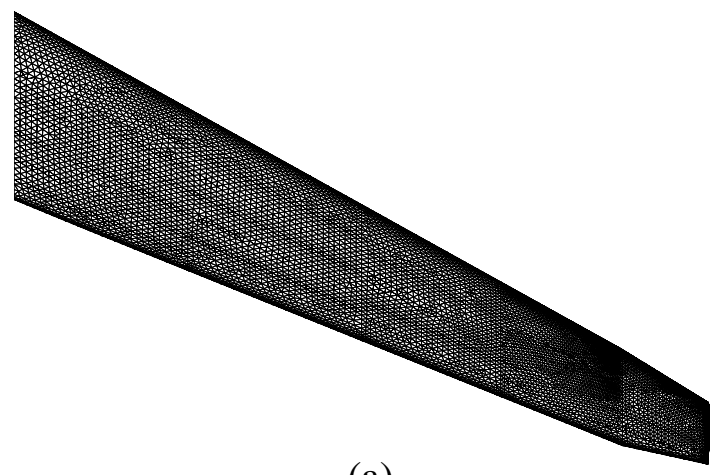

(a)

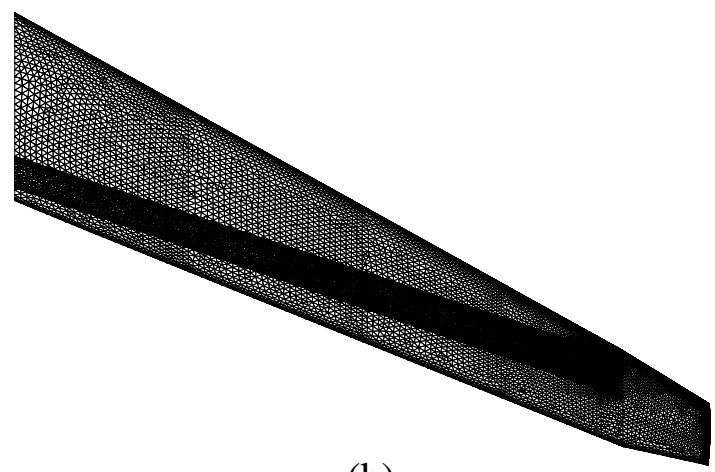

(b)

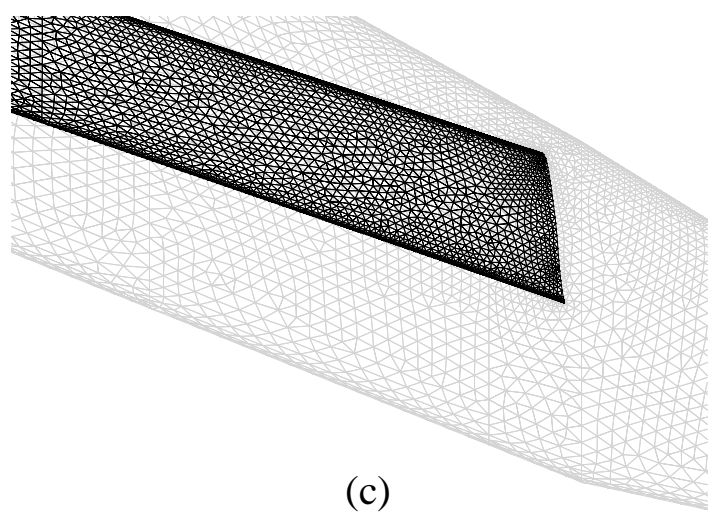

Figure 4.13: Surface grid on the SS5 strut-braced wing with a straight strut (a) Wing upper surface (b) Wing and strut lower surfaces (c) Close-up view of the wing-strut junction

Table 4.3: Grid characteristics for the various SS5 strut-braced wing configurations

\begin{tabular}{|l|c|c|}
\hline Configuration & $\begin{array}{c}\text { Number of surface } \\
\text { triangles }\end{array}$ & $\begin{array}{c}\text { Total number } \\
\text { of cells }\end{array}$ \\
\hline \hline Clean wing & 24,276 & 941,932 \\
Arch with $R=0 \mathrm{ft}$ (straight strut) & 55,546 & $1,647,086$ \\
Arch with $R=1 \mathrm{ft}$ & 46,824 & $1,708,717$ \\
Arch with $R=2 \mathrm{ft}$ & 48,796 & $1,842,099$ \\
Arch with $R=3 \mathrm{ft}$ & 50,440 & $1,879,482$ \\
Arch with $R=4 \mathrm{ft}$ & 49,724 & $1,923,555$ \\
\hline
\end{tabular}



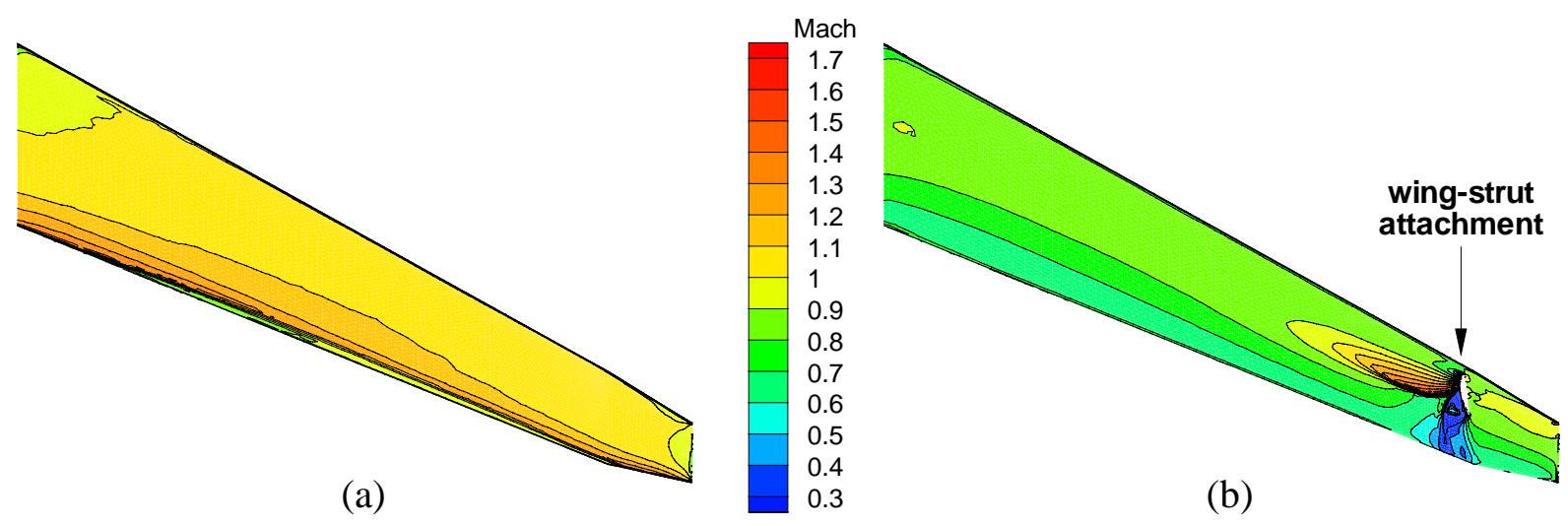

(b)

Figure 4.14: Mach number contours on the SS5 strut-braced wing with a straight strut, $M_{\infty}=0.85, \alpha_{\text {root }}=2.36^{\circ}\left(\alpha=-3.97^{\circ}\right)$ (a) Wing upper surface (b) Wing lower surface (the strut has been removed from the figure to show the contours on the wing surface)

contours on the upper surface of the clean wing shown in Figure 4.9. However, on the lower surface, the straight strut has a more dramatic effect on the flow than the arch-shaped strut. A region of high speed flow extends far inboard of the straight strut. This is due to the restricted channel flow near the junction and the low wing-strut intersection angle, but also to the footprint of the junction. The footprint is highly cambered even though a symmetric airfoil section was used for the strut. The flow accelerates very rapidly along the strut surface in the vicinity of the junction. For the arch-shaped strut arrangement, such is not the case. The footprint of the intersection is a symmetric airfoil. The flow on the wing is barely disturbed by the presence of the strut and the flow is not accelerated too dramatically along the strut surface.

The spanload distribution for the various strut arrangements is presented in Figure 4.10 compared to the spanload of the wing alone. The lift distribution for the wing of the SS5 design deviates significantly from the elliptic distribution in the vicinity of the wing-strut junction. The presence of the strut disrupts the flowfield and causes a reduction of the lift locally. As the arch radius is increased, the distance between the wing and the strut is increased and the load becomes closer to the lift distribution of the clean wing. Outboard of the wing-strut junction, all the lift distributions are similar. On the strut, the load is small between $\eta=0 \%$ and $\eta=50 \%$. The curves for the various cases fall on top of each other. An interesting thing to note is that the strut is negatively loaded in that range. As we move closer to the junction, the distance between the strut and the wing is reduced. The fluid 

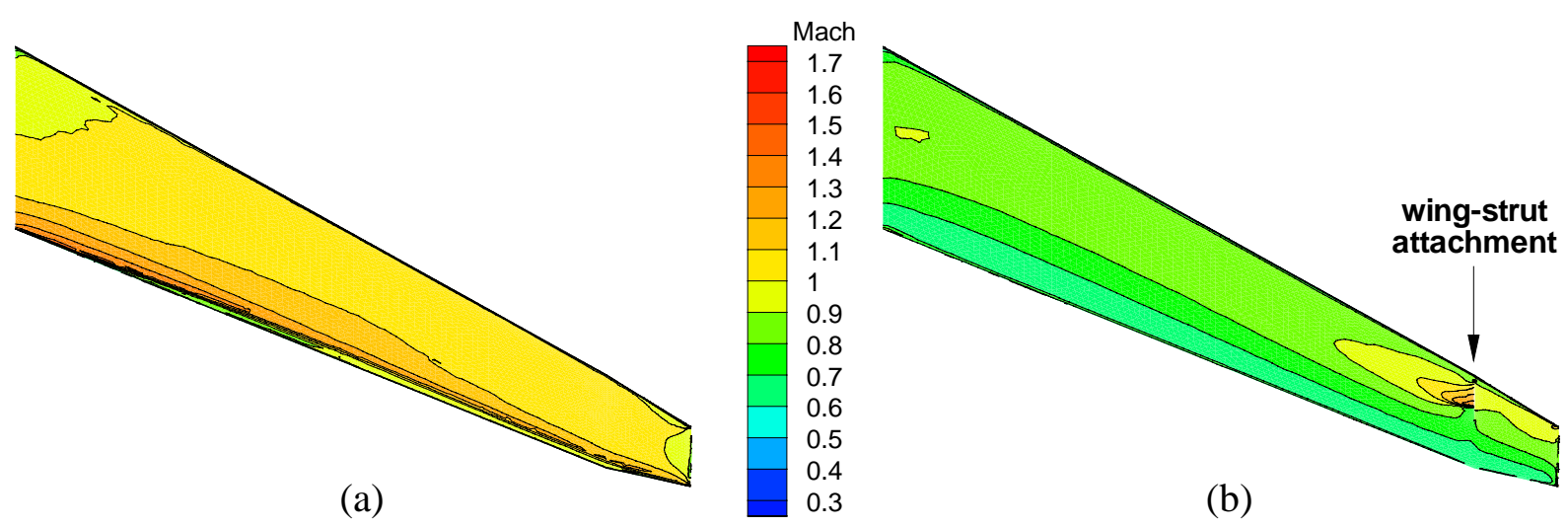

(b)

Figure 4.15: Mach number contours on the SS5 strut-braced wing with a 4-ft arch, $M_{\infty}=0.85, \alpha_{\text {root }}=2.36^{\circ}\left(\alpha=-3.97^{\circ}\right)$ (a) Wing upper surface (b) Wing lower surface (the strut has been removed from the figure to show the contours on the wing surface)

particles are accelerated in the channel, causing the pressure to drop. Therefore, the load is increased on the strut and reduced on the wing. The load is positive on the strut in that region.

The pressure distribution on the main wing is shown in Figure 4.16. Between $\eta=0 \%$ and $\eta=50 \%$, there is no obvious difference between the pressure on the clean wing and on the wing of any of the SS5 designs. A similar observation can be made of the flow characteristics outboard of the junction. However, the influence of the strut can be observed clearly between $\eta=70 \%$ and $\eta=85 \%$. The straight strut perturbs the flow a little more than the other cases. As noted earlier, the acceleration of the particles in the channel is accompanied by a drop in the pressure coefficient. Matters get worse as we get closer to the wing-strut junction. At $\eta=85 \%$, the case of the straight strut has a very strong shock at $x / c=0.30$ and a weaker one at $x / c=0.60$. This is in no way comparable to the other cases which exhibit only one shock at $x / c=0.45$.

The pressure distributions on the strut are pictured in Figure 4.17. At span stations up to and including $\eta=50 \%$, the strut is negatively loaded. When we get closer to the junction, the lift on the strut increases progressively. The incidence of the fluid particles on the strut is increased. The strut-braced wing with a straight strut is the only configuration to exhibit a shock for the span station $\eta=75 \%$. Right in the vicinity of the intersection, the shock for all cases gains strength. Again, the case with a straight strut shows a significant disturbance 


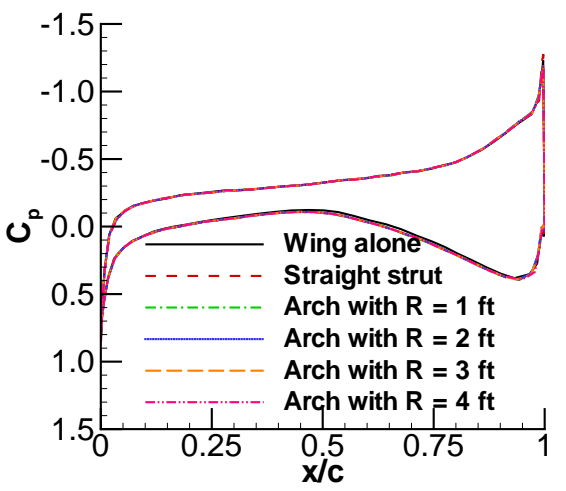

(a)

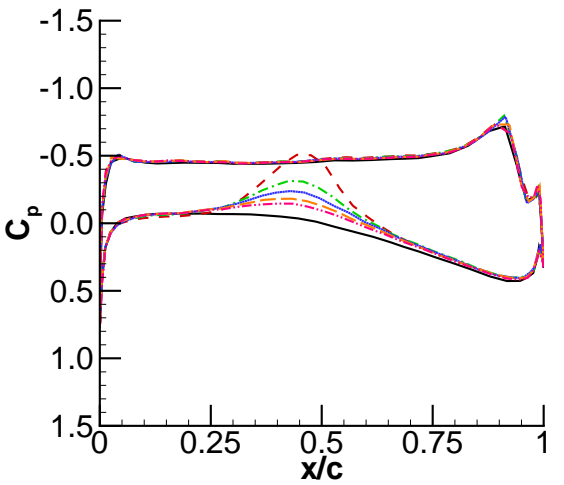

(d)

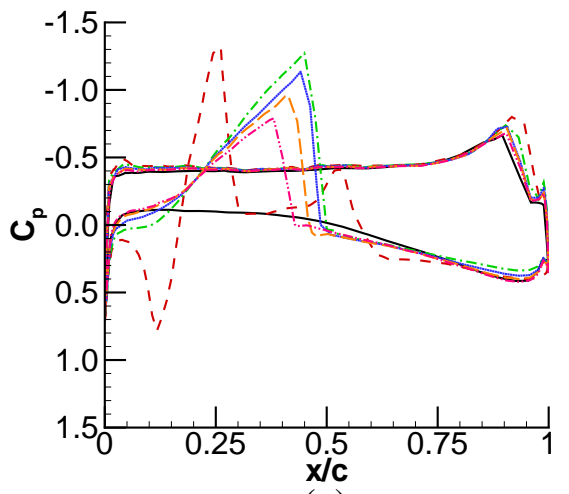

(g)

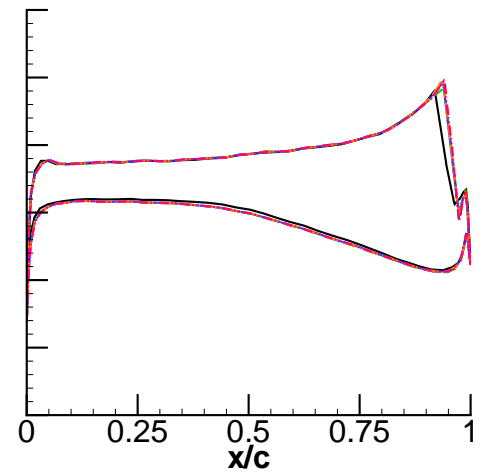

(b)

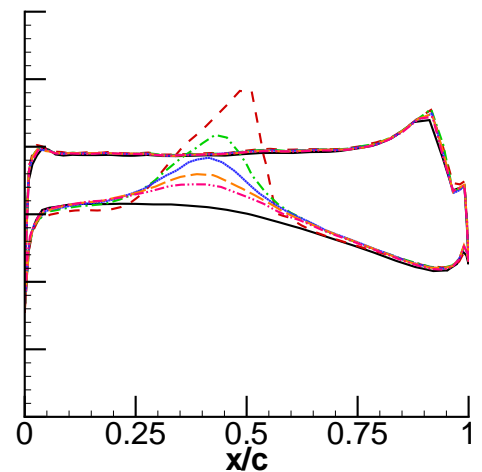

(e)

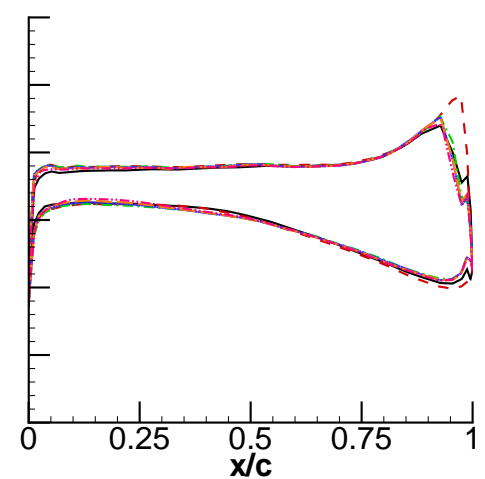

(h)

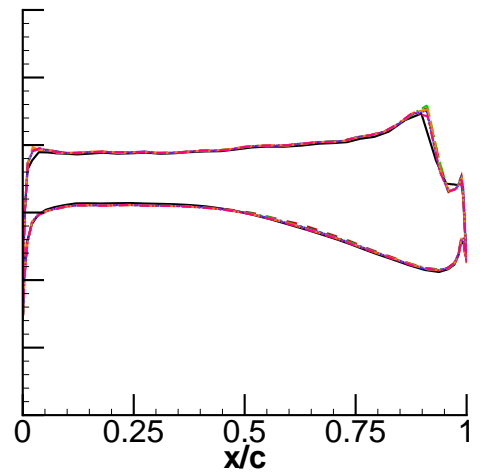

(c)

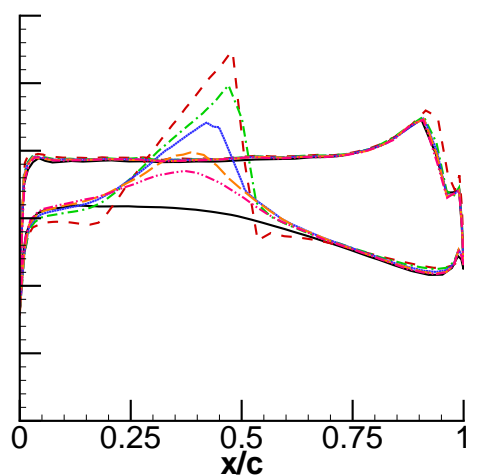

(f)

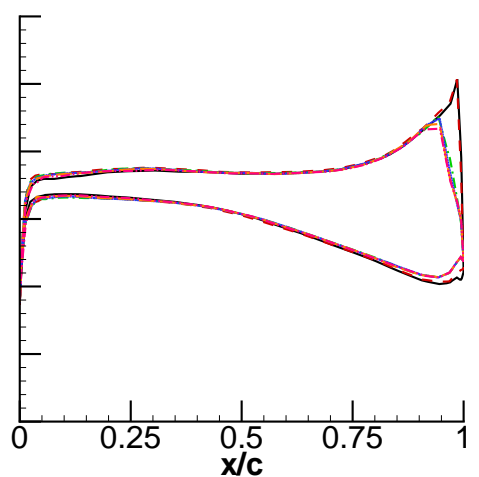

(i)

Figure 4.16: Influence of the strut on the wing pressure distribution for the SS5 strut-braced wing design, $M_{\infty}=0.85, \alpha_{\text {root }}=2.36^{\circ}\left(\alpha=-3.97^{\circ}\right)$ (a) $\eta=0 \%$ (b) $\eta=25 \%$ (c) $\eta=50 \%$ (d) $\eta=70 \%$ (e) $\eta=75 \%$ (f) $\eta=80 \%$ (g) $\eta=85 \%$ (h) $\eta=90 \%$ (i) $\eta=95 \%$ 


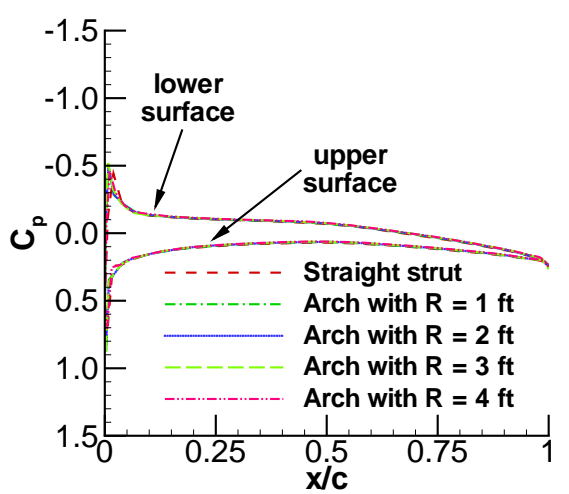

(a)

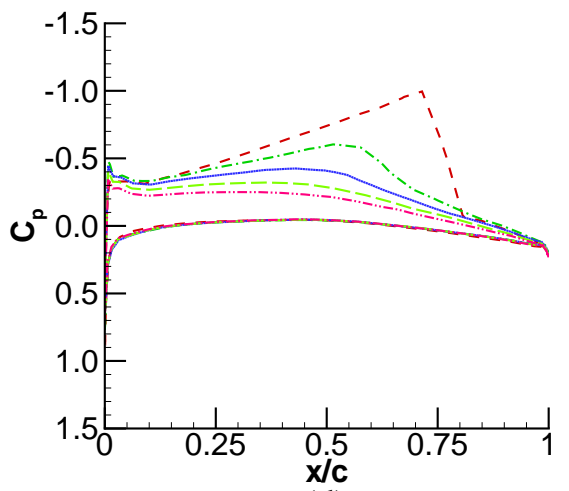

(d)

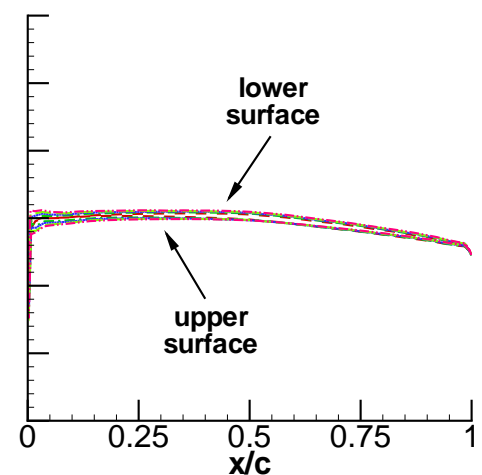

(b)

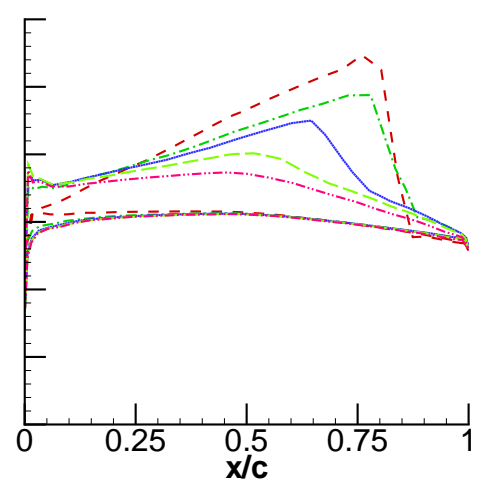

(e)

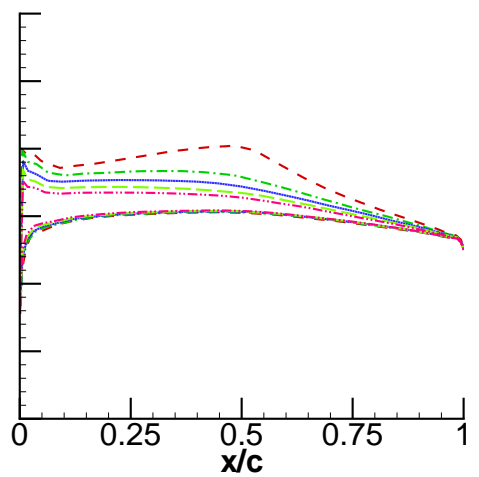

(c)

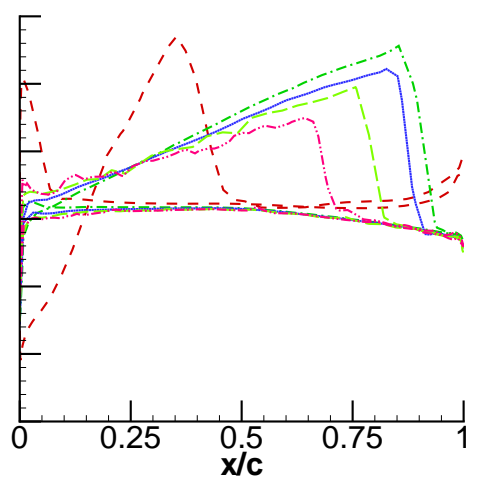

(f)

Figure 4.17: Pressure distribution on the strut of the SS5 strut-braced wing design, $M_{\infty}=0.85, \alpha_{\text {root }}=2.36^{\circ}\left(\alpha=-3.97^{\circ}\right)(\mathrm{a}) \eta=25 \%$ (b) $\eta=50 \%$ (c) $\eta=70 \%$ (d) $\eta=75 \%$ (e) $\eta=80 \%$ (f) $\eta=85 \%$

of the flowfield compared to the other cases. From these plots, it can be concluded that an arch with a radius of $1 \mathrm{ft}$ only has already some advantages over the straight strut because its influence on the flowfield is weaker.

The lift and drag coefficients for the various configurations are listed in Table 4.4. The drag increment $\Delta C_{D}$ of each configuration compared to the clean wing is shown in the last column of the table. This is obtained by subtracting the drag coefficient of the wing alone from the drag coefficient of the strut-braced wing design. That quantity gives an indication of how the drag penalty varies when the arch radius changes.

Despite the fact that the strut was rotated in order to have negligible incidence with respect to the flow, the strut carries some lift and changes the flow pattern on the wing surface. That explains why the lift coefficient is not constant for all cases in Table 4.4. 
Table 4.4: Lift and drag coefficients of the SS5 strut-braced wing configurations, $M_{\infty}=0.85$, $\alpha_{\text {root }}=2.36^{\circ}\left(\alpha=-3.97^{\circ}\right)$

\begin{tabular}{|l|c|c|c|}
\hline Configuration & $C_{L}$ & $C_{D}$ & $\Delta C_{D}$ \\
\hline \hline Clean wing & 0.5253 & 0.0121 & 0.0000 \\
\hline Arch with $R=0 \mathrm{ft}$ (straight strut) & 0.5396 & 0.0152 & 0.0031 \\
Arch with $R=1 \mathrm{ft}$ & 0.5330 & 0.0147 & 0.0026 \\
Arch with $R=2 \mathrm{ft}$ & 0.5308 & 0.0141 & 0.0021 \\
Arch with $R=3 \mathrm{ft}$ & 0.5300 & 0.0137 & 0.0017 \\
Arch with $R=4 \mathrm{ft}$ & 0.5282 & 0.0133 & 0.0013 \\
\hline
\end{tabular}

The drag coefficient is reduced steadily when the arch radius is increased from $R=0 \mathrm{ft}$ (straight strut) to $R=4 \mathrm{ft}$. The presence of the strut for the case where $R=0 \mathrm{ft}$ produces an increment of $26 \%$ of the drag coefficient compared to the clean wing. This could be anticipated from the strong shocks observed in the pressure distributions in Figures 4.16 and 4.17. The configuration with $R=4 \mathrm{ft}$ has $11 \%$ more drag than the clean wing case. This corresponds to a reduction of $59 \%$ of the drag increment $\Delta C_{D}$ compared to the strut-braced wing with $R=0 \mathrm{ft}$.

The drag penalty $\Delta C_{D}$ captured by the CFD solutions can be used as a design tool for MDO studies of the strut-braced wing designs. It is convenient to represent the drag penalty by fitting a curve in terms of the arch radius. A quadratic response surface can be obtained with the statistical software JMP [116] by employing a standard least-squares procedure:

$$
\Delta C_{D}=0.00315-0.000586 R+0.0000293 R^{2}
$$

where $R$ is in feet. The response surface has a coefficient of determination of 0.9991 and a root mean square error of $3.1 \times 10^{-5}$. The curve-fit is represented in Figure 4.18 with the data points from Table 4.4. As one can see, the response surface is in very good agreement with the CFD data.

\subsection{Concluding Remarks}

The use of inviscid CFD tools to study the aerodynamics of the strut-braced wing designs was successful in providing an estimate of the drag penalty associated with the presence of a straight or arch-shaped strut without any local shaping to reduce the interference drag. 


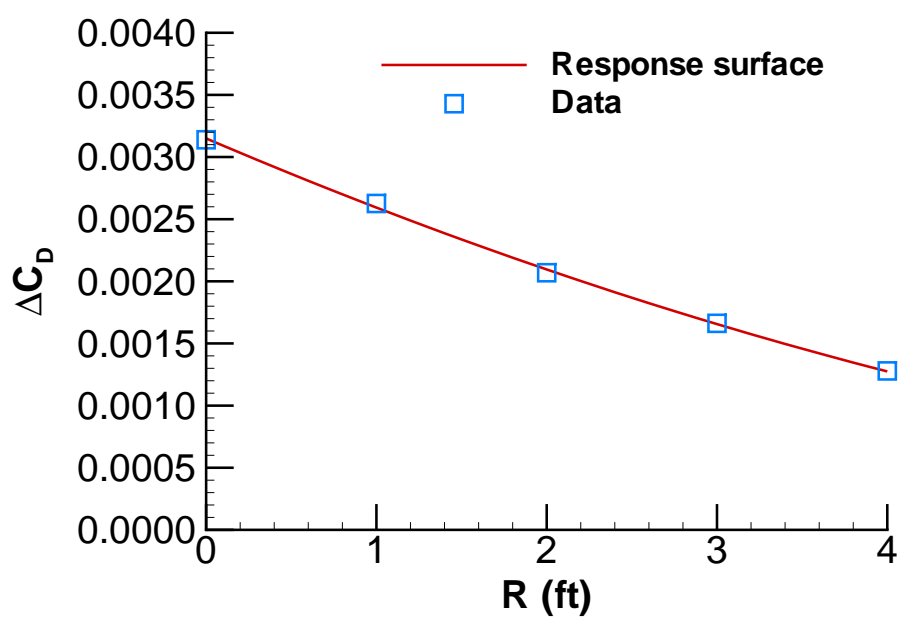

Figure 4.18: Interference drag penalty $\Delta C_{D}$ in terms of the arch radius, $M_{\infty}=0.85$, $\alpha_{\text {root }}=2.36^{\circ}$

The response surface developed gives a good estimate of the drag increment compared to the clean wing in terms of the arch radius. However, such a study is not complete because the effect of the viscosity, and variations in the strut chord, the intersection angle, and the thickness of the strut were not taken into account. A study focusing on those aspects for a more general configuration is presented in Chapter 5. It will be shown that the viscous effects have a favorable influence on the flow in most instances, because they alleviate the strength of the shocks near the intersection. In addition, no attempt was made at using fairings near the wing-strut and wing-wall intersections. In subsonic flow, Hoerner [24] notes that the employment of fairings can reduce the interference drag of a junction to $10 \%$ or less of its unfaired value. Thus, the results in Eq. 4.3 and in Figure 4.18 should be viewed as pessimistic and, therefore, conservative from an MDO perspective. 


\section{Chapter 5}

\section{Analysis of the Interference Drag of a Strut Inclined to a Surface Using Euler and Navier-Stokes Equations}

This chapter provides a methodology to predict the interference drag produced by a streamlined strut intersecting a flat surface in transonic flow. This is a generic case employed to simulate the flowfield in the vicinity of wing-strut, wing-pylon, and wing-body junctions. For a complete aircraft configuration, the accurate resolution of the flow near junctures would require thousands of nodes and a lot of computational resources. However, by considering the simplified strut-wall geometry, it is possible to use a very fine grid in the region near the junction and stay within acceptable limits in terms of computational cost.

In this chapter, the impact of the thickness to chord ratio of the strut, the Reynolds number, and the effect of the angle made by the strut with the wall are studied. First, the CFD tools employed are validated with experimental wind tunnel data. The strutwall problem is then described and the approach to calculate the interference drag for such configurations is detailed. The general conceptual method employed is similar to the one used by Hoerner [24] for experimental data in subsonic flow. Results are presented for viscous and inviscid calculations performed on several strut-wall arrangements. A response surface to estimate the interference drag is presented. Finally, the flowfield is investigated with pressure contours and streamtraces. 


\subsection{Modeling of the Problem}

To study the phenomenon at hand, unstructured grids were obtained with the grid generation programs AFLR2 [98, 99] and VGRIDns [100, 101]. The implicit, upwind, finite-volume flow solvers FUN2D and FUN3D [104, 105] from NASA Langley were used. These codes can solve the Euler and the Reynolds-averaged Navier-Stokes equations for compressible flows. They have been thoroughly tested, so they are suitable for use in the numerical "experiments" here. None of the calculations presented in this chapter employed a limiter to improve the convergence rate. For the viscous flows, the turbulence model of Spalart and Allmaras [106] is resolved to the wall without the use of wall functions because those lack accuracy in computing juncture flows. Since the main focus of this chapter is about the flow around strut-wall intersections, it makes sense to utilize a turbulence model that is integrated to the wall. The grid generation and flow solver tools were described at length in Chapter 3.

\section{$5.2 \quad$ Validation}

Since we are interested in studying the flow around a strut-wall configuration, we need to assess the accuracy of the three-dimensional flow solution methods to be used for such a case. An experiment was performed by Bartelheimer et al. [46] on a strut perpendicular to the walls of a wind tunnel. Pressure measurements were made along the span of the strut. Although no force measurements were made, the sectional normal force coefficient $C_{n}$ was computed at each span station, possibly by integrating the measured pressure distribution. The axial force coefficient was not available. The boundary layer characteristics were also determined along the walls to help reproduce the test conditions. It should be noted that the data is suitable only for "in-tunnel" CFD validation because the strut was enclosed by solid wind tunnel walls above, below and on the sides. The data was not corrected for the effect of blockage. The strut has a span $b=340 \mathrm{~mm}$ and a chord $c=200 \mathrm{~mm}$. The walls above and below the model are located symmetrically a distance of $300 \mathrm{~mm}$ away.

The numerical calculations were performed on a grid that takes advantage of the symmetry of the configuration. The strut was modeled with a span of $170 \mathrm{~mm}$ only, with a symmetry boundary condition applied to one of the side walls. The no-slip condition was enforced on the other walls and on the strut surface. The inflow plane is located $3000 \mathrm{~mm}$ upstream of the model in order to obtain a side wall boundary layer comparable to the one 
measured in the experiment. Downstream, the far-field boundary extends $2000 \mathrm{~mm}$ from the strut trailing edge. The first grid point is located $0.002 \mathrm{~mm}$ above the viscous surfaces to provide at least one grid point within the laminar sublayer. The grid point distribution employed was based on similar fine grids generated in Section 5.5. The final grid contains 23, 082 surface nodes, 300, 843 viscous nodes, and a total of 367, 516 nodes. The surface nodes represent all the nodes lying on the strut and on the faces of the computational box. The viscous nodes are the nodes generated in the viscous layers with the advancing-layers methodology [101].

The freestream Mach number is 0.73 , and the Reynolds number is $R e_{c}=6 \times 10^{6}$. The airfoil makes an angle of $1.5^{\circ}$ with respect to the incoming freestream velocity. The entire flowfield is considered as fully turbulent. The side wall boundary layer displacement thickness, $\delta^{*}$, and momentum thickness, $\theta$, are compared with the experimental values $1050 \mathrm{~mm}$ upstream of the strut leading edge in Table 5.1. There are three experimental sets of values because the boundary layer characteristics were measured during three series of wind tunnel tests. To compute $\delta^{*}$ and $\theta$ from the CFD solution at that streamwise location, the velocity and density profiles were extracted along a line midway between the top and bottom walls of the wind tunnel. The integration was then performed in a direction normal to the wall, from the wall surface to the centerline of the test section. The following definitions for $\delta^{*}$ and $\theta$ were employed:

$$
\begin{gathered}
\delta^{*}=\int_{0}^{c e n t e r}\left(1-\frac{\rho U}{\rho_{e} U_{e}}\right) d y \\
\theta=\int_{0}^{c e n t e r} \frac{\rho U}{\rho_{e} U_{e}}\left(1-\frac{\rho U}{\rho_{e} U_{e}}\right) d y
\end{gathered}
$$

where $U_{e}$ represents the edge velocity and $\rho_{e}$ denotes the edge density. The predicted values of $\delta^{*}$ and $\theta$ are $16 \%$ lower than the measurements. This can be explained by the fact that the grid spacing on the side walls is the same all the way from the inflow plane to the outflow plane. There was no attempt to have finer normal grid spacing near the inflow plane where the boundary layer is much thinner than further downstream. The growth of the boundary layer is, therefore, not captured precisely.

The calculated pressure distributions are shown compared to experimental measurements in Figure 5.1. It should be noted that the pressure distribution at $\eta=0.6 \%$ is located within the side wall boundary layer. For all locations, the agreement is good everywhere except on the upper surface near the shock. The shock is predicted too far from the leading 
Table 5.1: Side wall boundary layer dimensions $1050 \mathrm{~mm}$ upstream of the strut

\begin{tabular}{|c|c|c|c|c|}
\hline & $M_{\infty}$ & $R e_{c}$ & $\delta^{*}(\mathrm{~mm})$ & $\theta(\mathrm{mm})$ \\
\hline \hline Calculation & 0.730 & $6 \times 10^{6}$ & 2.75 & 2.16 \\
\hline \multirow{3}{*}{ Experiment } & 0.730 & $5.86 \times 10^{6}$ & 3.17 & 2.25 \\
& 0.729 & $5.83 \times 10^{6}$ & 3.21 & 2.50 \\
& 0.729 & $6.09 \times 10^{6}$ & 3.19 & 2.48 \\
\hline
\end{tabular}

Table 5.2: Normal force coefficient $C_{n}$ along the wing span

\begin{tabular}{|c|c|c|}
\hline Station & Calculation & Experiment \\
\hline \hline $\mathrm{y}=2 \mathrm{~mm}(\eta=0.6 \%)$ & 0.6974 & 0.6651 \\
$\mathrm{y}=10 \mathrm{~mm}(\eta=2.9 \%)$ & 0.7074 & 0.6830 \\
$\mathrm{y}=20 \mathrm{~mm}(\eta=5.9 \%)$ & 0.7186 & 0.6885 \\
$\mathrm{y}=40 \mathrm{~mm}(\eta=11.8 \%)$ & 0.7298 & 0.7127 \\
$\mathrm{y}=80 \mathrm{~mm}(\eta=23.5 \%)$ & 0.7346 & 0.7186 \\
$\mathrm{y}=170 \mathrm{~mm}(\eta=50.0 \%)$ & 0.7382 & 0.7205 \\
\hline
\end{tabular}

edge of the strut. For all cases, the pressure on the strut lower surface is in almost perfect agreement with the experimental data. In light of these quite favorable results, we can be confident in applying the proposed tools to the modeling of the flow near the junction.

The normal force coefficient $C_{n}$ computed at each span station along the wing is compared against the experimental data in Table 5.2. Because the flow solution did not predict the shock at the same location as in the experiment, that discrepancy causes the calculation to overestimate the experimental value of the normal force coefficient. However, the agreement is generally good. The experimental data shows that the value of the normal force coefficient increases with the distance from the side wall. That trend is captured by the CFD solution as well.

\subsection{Problem Description}

The objective of the study at hand is to determine the interference drag of simple intersections in transonic flow. To simulate the effect of a wing-strut or a wing-pylon junction, the case of the flow around a strut between parallel walls was analyzed. Figure 5.2 depicts the configuration of interest. The strut is enclosed between two side walls, and the other faces 


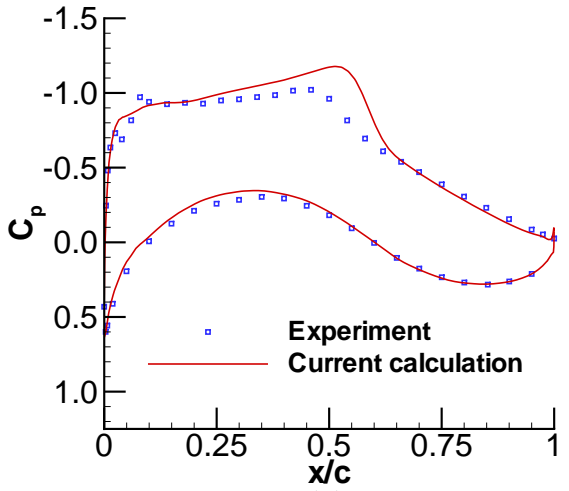

(a)

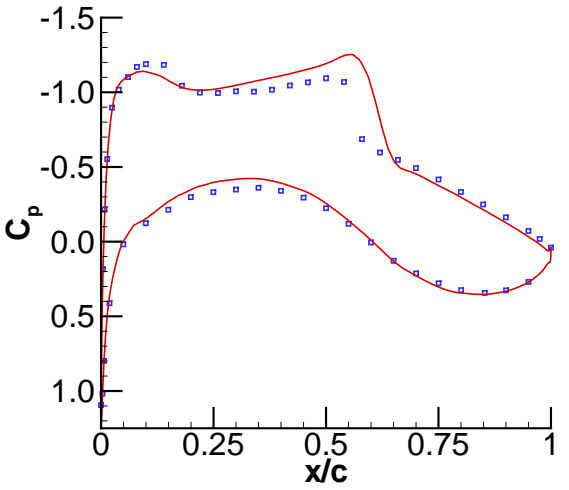

(d)

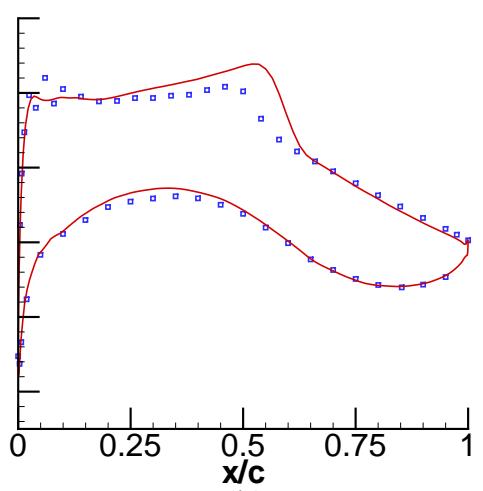

(b)

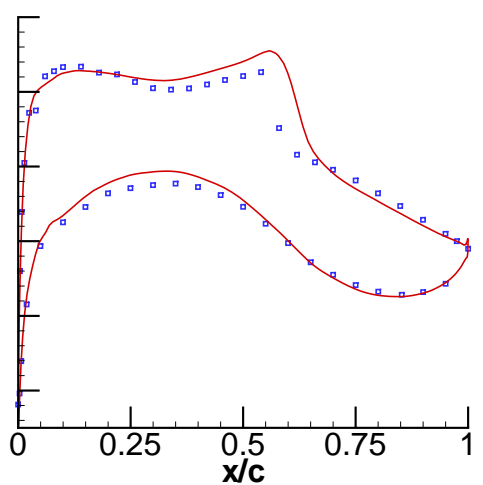

(e)

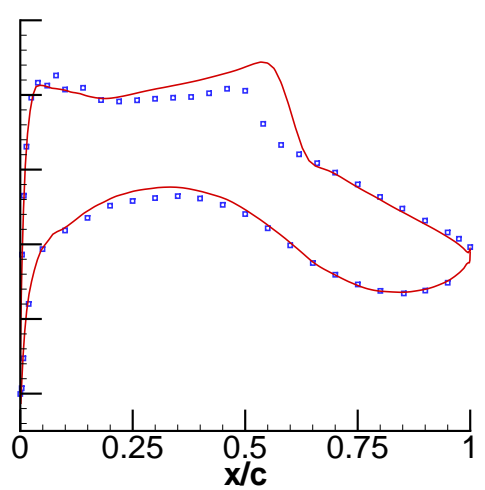

(c)

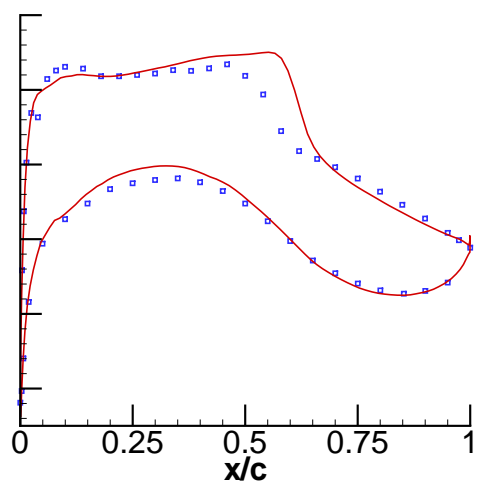

(f)

Figure 5.1: $C_{p}$ distribution for the AGARD test case, $M_{\infty}=0.73, \alpha=1.5^{\circ}, R e_{c}=6 \times 10^{6}$ (a) $\mathrm{y}=2 \mathrm{~mm}(\eta=0.6 \%)(\mathrm{b}) \mathrm{y}=10 \mathrm{~mm}(\eta=2.9 \%)(\mathrm{c}) \mathrm{y}=20 \mathrm{~mm}(\eta=5.9 \%)$ (d) y $=40 \mathrm{~mm}(\eta=11.8 \%)(\mathrm{e}) \mathrm{y}=80 \mathrm{~mm}(\eta=23.5 \%)(\mathrm{f}) \mathrm{y}=170 \mathrm{~mm}(\eta=50.0 \%)$ 


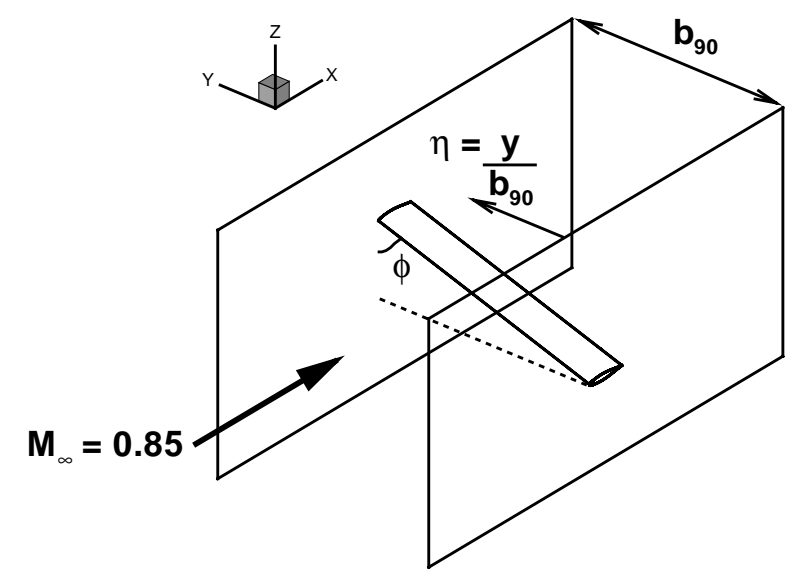

Figure 5.2: Strut between parallel walls in transonic flow

of the computational box are open to the air. The domain extends 10 strut chords to the far-field boundary. The distance between the parallel walls is set to $b_{90}=5$ strut chords to achieve two-dimensional flow at mid span. The angle $\phi$ made by the strut with the adjacent wall was varied from $90^{\circ}$ to $60^{\circ}$ and $30^{\circ}$.

The configurations were analyzed for a freestream Mach number of $M_{\infty}=0.85$ and an altitude of 40,000 ft, which are typical cruise conditions for a civil transport airplane. These yield a Reynolds number per meter of $5.3 \times 10^{6}$. For typical strut chords of 1 and 2 meters, the corresponding Reynolds numbers are $5.3 \times 10^{6}$ and $10.6 \times 10^{6}$. The strut section makes an angle of attack $\alpha=0^{\circ}$ with respect to the freestream flow for all the calculations. To avoid loading the strut as much as possible and to assess the effect of varying the thickness of the strut, symmetric airfoil sections with $t / c$ of $5 \%$ and $7.5 \%$ were studied. The strut sections were obtained from the airfoil generation code LADSON [117] for the NACA 64A- series. The airfoil denomination NACA 64A005 and NACA 64A007.5 will be used to identify the two sections.

\subsection{Calculation of the Interference Drag}

The approach to determining the interference drag is to make use of the CFD tools as if one were performing an experiment in a wind tunnel. First, solve the three-dimensional Navier- 
Stokes equations for the flow around a given strut-wall $(S+W)$ arrangement to determine $C_{L_{S+W}}$ and $C_{D_{S+W}}$ defined as:

$$
\begin{gathered}
C_{L_{S+W}}=\frac{L_{S+W}}{q_{\infty} S_{r e f}} \\
C_{D_{S+W}}=\frac{D_{S+W}}{q_{\infty} S_{r e f}}
\end{gathered}
$$

where the dynamic pressure is $q_{\infty}=\frac{\gamma}{2} p_{\infty} M_{\infty}^{2}$. We obtain $C_{L_{S+W}}$ and $C_{D_{S+W}}$ by integrating the pressure and skin friction over the strut surface only. The wall surface is not considered in the integration of the viscous forces.

The reference area used to non-dimensionalize the coefficients is determined in terms of the chord and the total length of the strut. Since the distance between the walls is set to 5 chords, it follows from Figure 5.2 that:

$$
S_{\text {ref }}=\frac{5 c^{2}}{\sin \phi}
$$

Hence, the definitions of the lift and drag coefficients become:

$$
\begin{aligned}
C_{L_{S+W}} & =\frac{L_{S+W}}{q_{\infty}}\left(\frac{\sin \phi}{5 c^{2}}\right) \\
C_{D_{S+W}} & =\frac{D_{S+W}}{q_{\infty}}\left(\frac{\sin \phi}{5 c^{2}}\right)
\end{aligned}
$$

A second Navier-Stokes analysis is performed to determine the lift and drag coefficients of the strut alone without the effect of the walls, namely $C_{L_{S}}$ and $C_{D_{S}}$. This can be achieved using the flow solver in one of two ways:

1. Solve the two-dimensional equations for the flow around the 2-D airfoil section of the strut

2. Consider the strut-wall arrangement with $\phi=90^{\circ}$ and apply the no-penetration, slip condition on the side walls, rather than the no-slip condition. The configuration will be equivalent to a strut with symmetry planes at both ends. Hence, the strut is considered as having an infinite span in this case, just like when the analysis is performed on a 2-D airfoil.

The second alternative yields:

$$
C_{D_{S}}=\frac{D_{S}}{q_{\infty}}\left(\frac{1}{5 c^{2}}\right)
$$


Twice the interference drag is calculated as the difference between the drag of the strut with the two walls and the drag of the strut without the effect of the walls. The result is nondimensionalized with respect to a conveniently chosen reference area to yield the interference

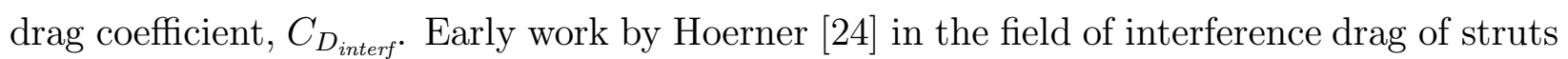
suggests several possibilities for the choice of the reference area such as $S_{\text {ref }}=t^{2}$ or $S_{\text {ref }}=c^{2}$ where $t$ and $c$ are the thickness and the chord of the strut respectively. In this study, we opted for the latter expression.

Since the configuration depicted in Figure 5.2 contains two wall-strut intersections, we will have to take one half of the drag difference:

$$
D_{\text {interf }}=\frac{1}{2}\left(D_{S+W}-D_{S}\right)
$$

The interference drag coefficient for one junction becomes:

$$
C_{D_{\text {interf }}}=\frac{D_{\text {interf }}}{q_{\infty} c^{2}}=\frac{1}{2}\left(\frac{D_{S+W}-D_{S}}{q_{\infty} c^{2}}\right)
$$

It should be noted that since the analyses are performed at $\alpha=0^{\circ}$ on symmetric airfoil sections, the numerical results should be such that $C_{L_{S+W}} \approx 0$ and $C_{L_{S}} \approx 0$.

As mentioned before, $C_{D_{S}}$ of the strut without the wall effect can be determined from a two-dimensional or a three-dimensional flow solution. Because of the large memory required for 3-D calculations compared to their 2-D counterparts, it is desirable to use a reduced number of mesh points. However, this comes with the price of a loss of accuracy of the solution. This penalty can be assessed by first solving the flow on a very fine grid around a 2-D airfoil. Then, using a 3-D grid with a more realistic number of nodes, the flow is solved for a strut with the same section placed perpendicularly between inviscid side walls. The solutions can be compared to evaluate the magnitude of the discretization error. The result of using this approach will be shown in the next section.

\subsection{Results and Discussion}

In this section, the results are presented for three-dimensional analyses performed on complete strut-wall configurations. The effects of the strut thickness $t / c$, the strut-wall angle $\phi$, and the Reynolds number $R e_{c}$ on the interference drag are determined from first Euler and then Navier-Stokes calculations. Pressure distributions are used to highlight the most important features of the flow. 
Table 5.3: Grid characteristics

\begin{tabular}{|c|c||c|c||c|c|c|}
\hline \multirow{2}{*}{$\begin{array}{c}\text { NACA } \\
\text { airfoil }\end{array}$} & \multirow{2}{*}{ Intersection angle $\phi$} & $\begin{array}{c}\text { Number of } \\
\text { surface } \\
\text { nodes }\end{array}$ & $\begin{array}{c}\text { Total } \\
\text { number } \\
\text { of nodes }\end{array}$ & $\begin{array}{c}\text { Number of } \\
\text { surface } \\
\text { nodes }\end{array}$ & $\begin{array}{c}\text { Number of } \\
\text { viscous } \\
\text { nodes }\end{array}$ & $\begin{array}{c}\text { Total } \\
\text { number } \\
\text { of nodes }\end{array}$ \\
\hline \multirow{4}{*}{64 A005 } & 2 -D & 576 & 9,905 & 576 & N/A & 27,615 \\
\cline { 2 - 7 } & $90^{\circ}$ (inviscid side walls) & 26,024 & 257,903 & 32,544 & 405,863 & 588,829 \\
& $90^{\circ}$ & 26,024 & 257,903 & 30,780 & 505,866 & 682,195 \\
& $60^{\circ}$ & 41,815 & 485,486 & 45,085 & 990,585 & $1,327,931$ \\
& $30^{\circ}$ & 62,107 & 487,793 & 61,819 & $1,377,022$ & $1,616,997$ \\
\hline \hline & $2-\mathrm{D}$ & 576 & 9,873 & 576 & N/A & 27,735 \\
\cline { 2 - 7 } 64A007.5 & $90^{\circ}$ (inviscid side walls) & 20,721 & 189,377 & 26,205 & 325,852 & 453,215 \\
& $90^{\circ}$ & 20,721 & 189,377 & 25,022 & 408,515 & 529,717 \\
& $60^{\circ}$ & 32,543 & 351,674 & 35,753 & 780,657 & $1,015,709$ \\
& $30^{\circ}$ & 47,086 & 392,929 & 41,076 & 922,279 & $1,071,661$ \\
\hline
\end{tabular}

\subsubsection{Euler Calculations}

For each strut-wall angle $\phi$, the Euler equations were solved on inviscid grids generated for the NACA 64A005 and NACA 64A007.5 airfoil sections. For each strut section, although the geometries are different, a similar grid point distribution was used to make sure the results are consistent. Grid stretching along the length of the strut was used more extensively away from the wall. The case where $\phi=90^{\circ}$ can be compared with the results of a 2-D analysis performed on the same airfoil section. The number of surface nodes and the total number of nodes for each case are presented in Table 5.3. The surface nodes for the two-dimensional cases represent the number of nodes on the complete airfoil and on the far-field boundary. For the $\phi=90^{\circ}$ case, the 3-D model has a span of only 2.5 chords because of the symmetry of the geometry.

In Chapter 4, the flow solutions obtained with USM3D were stopped after the residual had been reduced by three orders of magnitude, corresponding to the convergence of the lift and drag coefficients. However, FUN2D and FUN3D employ a different finite-volume formulation and it is necessary to do this exercise again to find the convergence criterion that is most suitable to the solutions obtained with those codes. The convergence criterion employed for the Euler calculations presented in this section is based on the convergence history for the NACA 64A007.5 strut section with $\phi=30^{\circ}$. This case was chosen because it is the most difficult configuration to analyze with the flow solver and it is likely to have more difficulty reaching convergence. The convergence history for that case is shown in Figure 5.3. The 


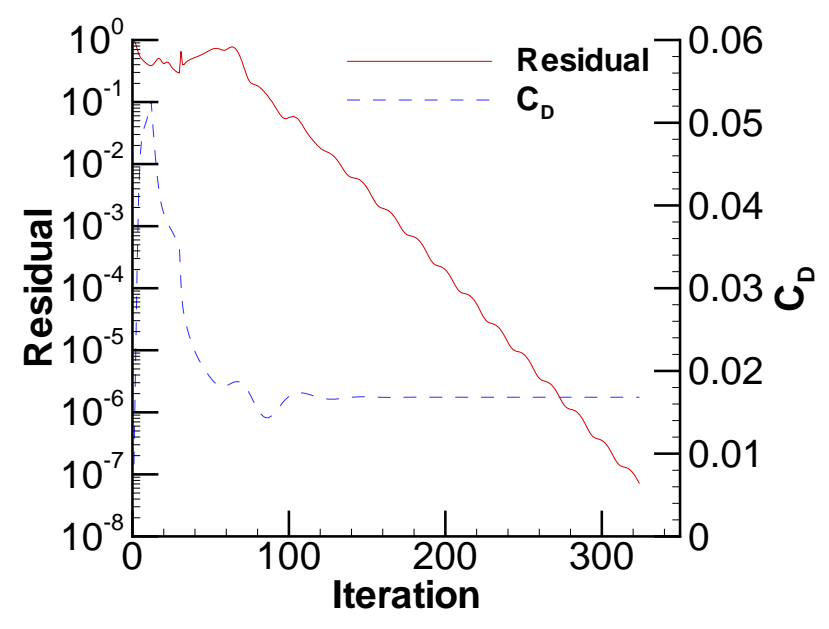

Figure 5.3: Convergence History NACA 64A007.5 - $\phi=30^{\circ}, M_{\infty}=0.85, \alpha=0^{\circ}$ (Euler solution) - Residual (left axis) and total drag coefficient (right axis)

drag coefficient doesn't vary much after the residual is reduced by four orders of magnitude. This convergence criterion is applied to all the Euler results shown in this section. The same criterion will be found satisfactory for the Navier-Stokes solutions in Section 5.5.2.

The pressure distributions along cross sections parallel to the walls are portrayed in Figure 5.4 for the NACA 64A005 section and in Figure 5.5 for the NACA 64A007.5 section. For the NACA 64A005 section, the flow is severely disrupted near the wall for $\phi=60^{\circ}$ and $\phi=30^{\circ}$. The proximity of the side wall creates a channel that forces the fluid to reach higher a velocity compared to the $\phi=90^{\circ}$ case. Although the pressure distribution for $\phi=90^{\circ}$ doesn't exhibit a shock, there is a strong shock for the $\phi=60^{\circ}$ and $\phi=30^{\circ}$ cases. The influence of the wall decays rapidly away from the wall. It is barely noticeable at $\eta=50 \%$ where the $C_{p}$ curves almost fall on top of the $\phi=90^{\circ}$ curves.

For the NACA 64A007.5 section, the reduction of the strut-wall angle $\phi$ severely disrupts the flowfield in the region near the wall. The shock for $\phi=60^{\circ}$ and $\phi=30^{\circ}$ is a lot stronger than for $\phi=90^{\circ}$. Its effect can also be felt a large distance away from the wall. Even at $\eta=50 \%$, the disturbance can be seen as the pressure distributions deviate significantly from the reference curve $\phi=90^{\circ}$.

The spanload distribution on the strut is pictured in Figure 5.6. The strut is highly loaded in the vicinity of the junction with the wall. The load distribution is antisymmetric with respect to mid span, at which point it is zero. The effect of the thickness is obvious 


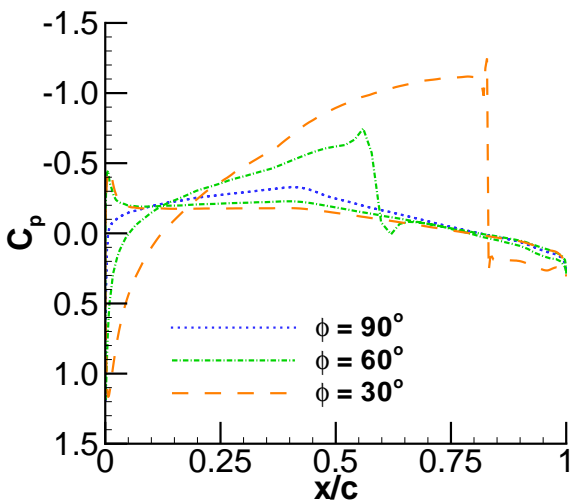

(a)

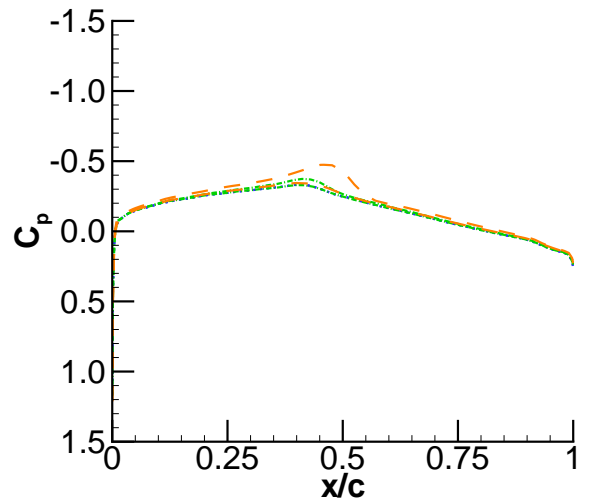

(d)

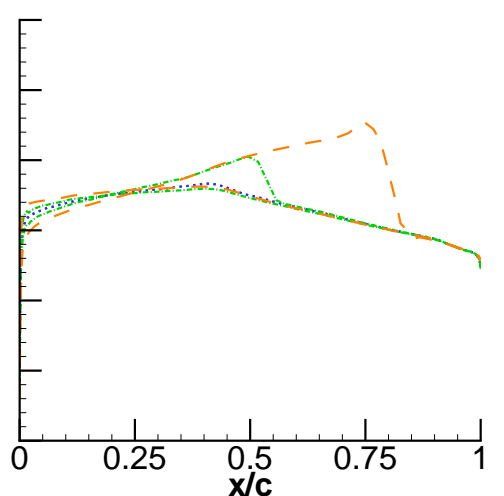

(b)

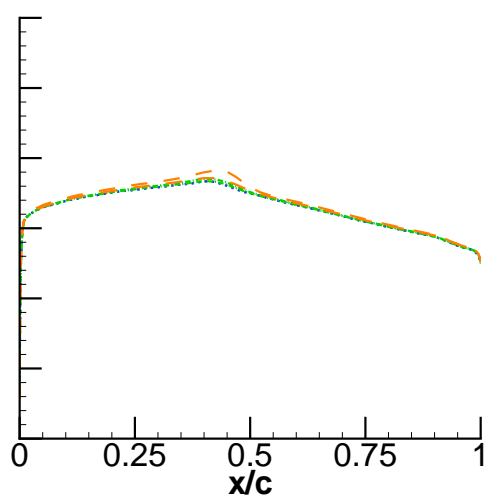

(e)

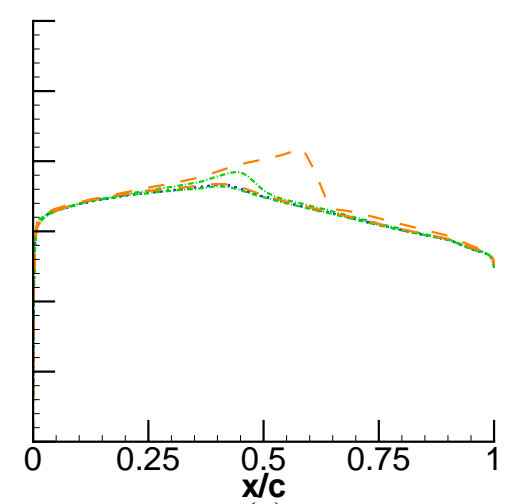

(c)

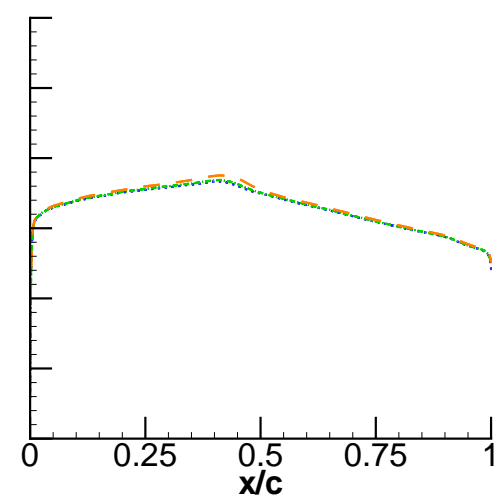

(f)

Figure 5.4: $C_{p}$ distribution for the NACA 64A005 strut, $M_{\infty}=0.85, \alpha=0^{\circ}$ (Euler solution) (a) $\eta=0 \%$ (b) $\eta=10 \%$ (c) $\eta=20 \%$ (d) $\eta=30 \%$ (e) $\eta=40 \%$ (f) $\eta=50 \%$ 


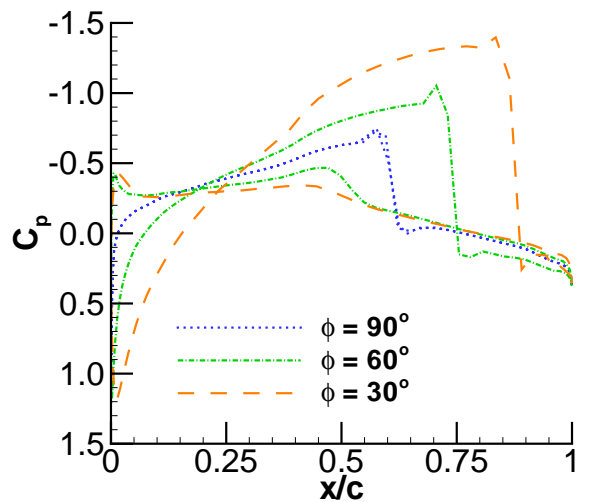

(a)

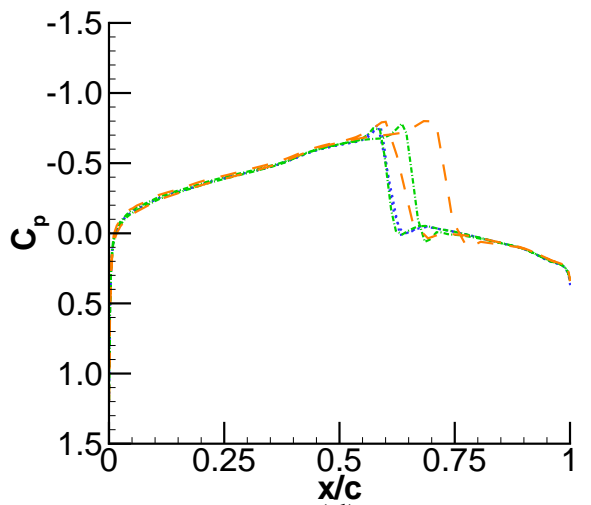

(d)

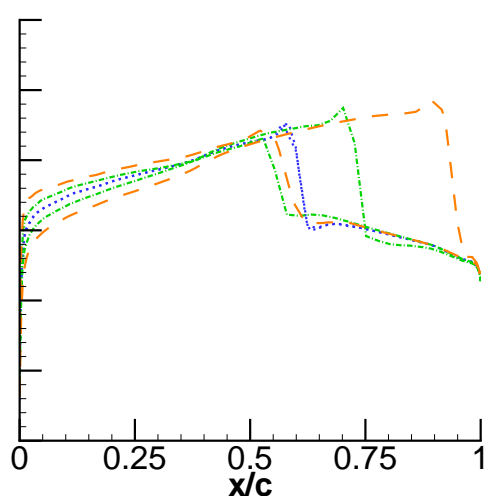

(b)

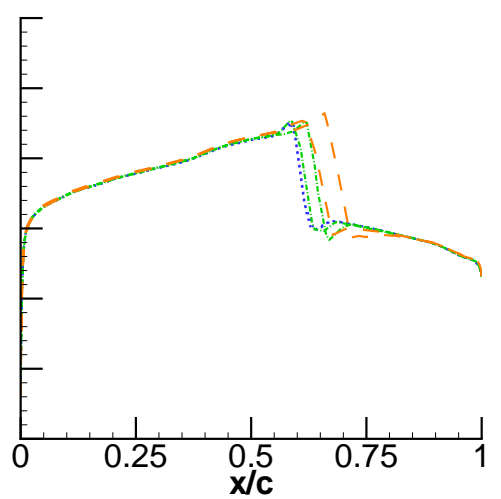

(e)

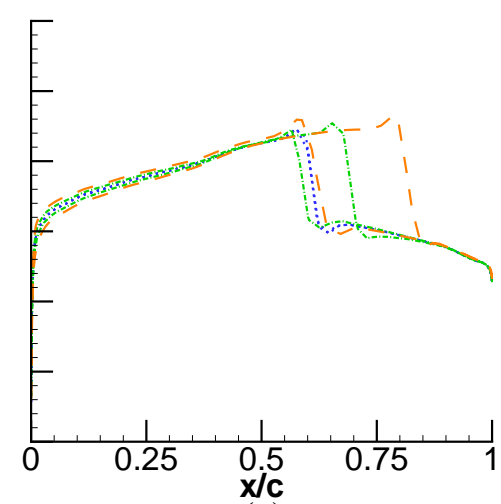

(c)

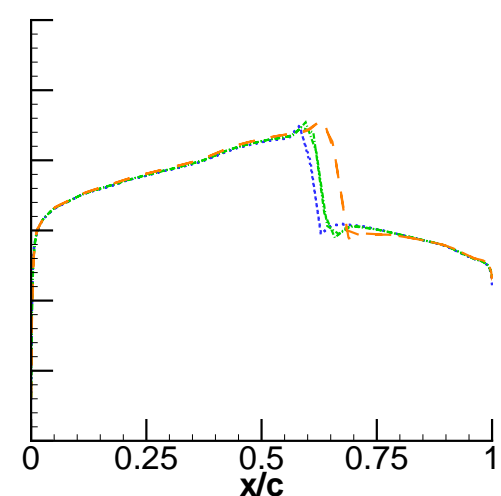

(f)

Figure 5.5: $C_{p}$ distribution for the NACA 64A007.5 strut, $M_{\infty}=0.85, \alpha=0^{\circ}$ (Euler solution) (a) $\eta=0 \%$ (b) $\eta=10 \%$ (c) $\eta=20 \%$ (d) $\eta=30 \%$ (e) $\eta=40 \%$ (f) $\eta=50 \%$ 


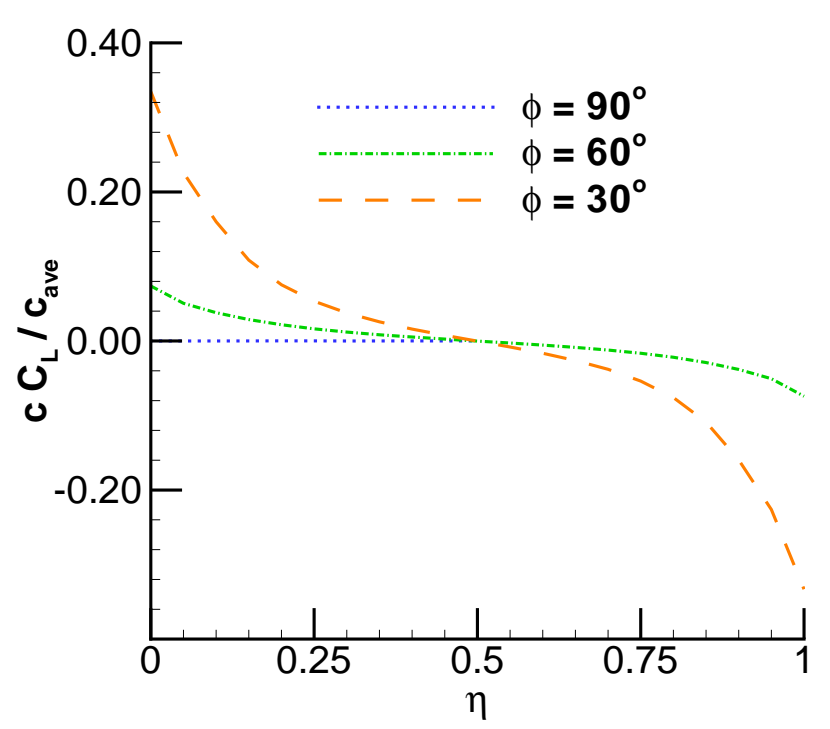

(a)

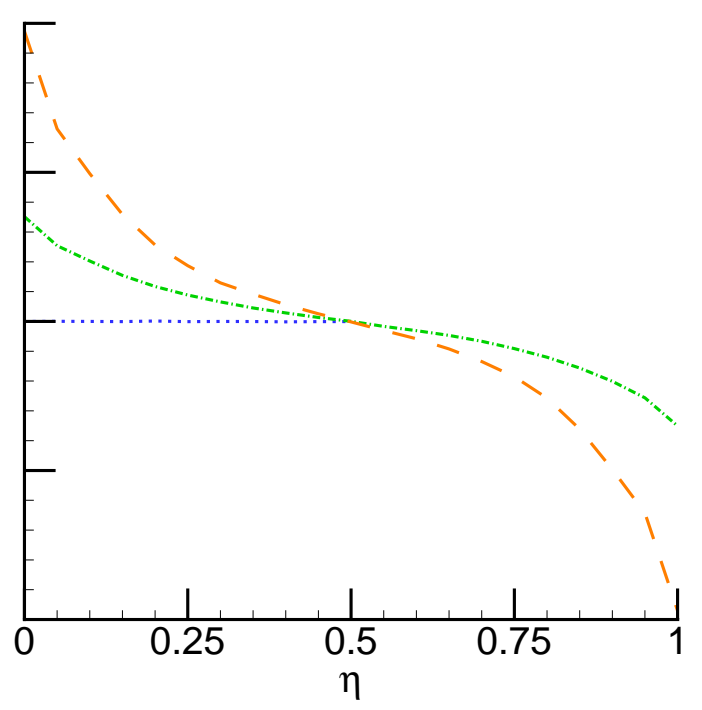

(b)

Figure 5.6: Spanload distribution, $M_{\infty}=0.85, \alpha=0^{\circ}$ (Euler solution) (a) NACA 64A005 (b) NACA 64A007.5

as the NACA 64A007.5 section shows a higher load than the NACA 64A005 over the span range. The load for $\phi=90^{\circ}$ is negligible. It goes from $\eta=0 \%$ to $\eta=50 \%$ only because half the configuration was modeled due to symmetry. The effect of reducing the strut-wall angle $\phi$ from $90^{\circ}$ to $60^{\circ}$ and $30^{\circ}$ is accompanied by a variation of the lift locally. Near the root where the load is positive, the lift increases, whereas at the strut tip, the strut becomes more negatively loaded.

The drag coefficients obtained for the two-dimensional and the three-dimensional analyses are shown in Table 5.4. For all cases, the magnitude of the lift coefficient is less than $3 \times 10^{-4}$. The drag comes out to be very close for the $2-\mathrm{D}$ and the $3-\mathrm{D}$ analyses for $\phi=90^{\circ}$. For the NACA 64A005 section, the drag is very small for $\phi=90^{\circ}$ because there is no shock along the strut surface. When the angle is reduced to $\phi=60^{\circ}$, the shock near the root observed in the pressure distribution in Figure 5.4 is responsible for increasing the drag coefficient slightly compared to the $\phi=90^{\circ}$ configuration. For $\phi=30^{\circ}$, the pressure distribution indicated the presence of a much stronger shock than for the $\phi=60^{\circ}$ case. The large increase in drag can be explained by the fact that the shock persists over almost $20 \%$ of the strut span, although rendered much weaker as the distance from the wall increases. For the NACA 64A007.5 strut section, the $\phi=90^{\circ}$ arrangement has a large drag penalty because 
Table 5.4: Drag coefficients at $M_{\infty}=0.85$

\begin{tabular}{|c|c||c||c|c|c||c|c|c|}
\hline \multirow{2}{*}{$\begin{array}{c}\text { NACA } \\
\text { airfoil }\end{array}$} & \multicolumn{1}{|c||}{ Intersection angle $\phi$} & Euler & \multicolumn{2}{c||}{$R e_{c}=5.3 \times 10^{6}$} & \multicolumn{3}{c|}{$R e_{c}=10.6 \times 10^{6}$} \\
\cline { 2 - 8 } & & $C_{D_{\text {tot }}}$ & $C_{D_{p}}$ & $C_{D_{v}}$ & $C_{D_{\text {tot }}}$ & $C_{D_{p}}$ & $C_{D_{v}}$ & $C_{D_{\text {tot }}}$ \\
\hline & $2-\mathrm{D}$ & 0.0001 & 0.0012 & 0.0060 & 0.0072 & 0.0011 & 0.0055 & 0.0066 \\
\cline { 2 - 8 } $64 \mathrm{~A} 005$ & $90^{\circ}$ (inviscid side walls) & 0.0002 & 0.0014 & 0.0058 & 0.0072 & 0.0013 & 0.0053 & 0.0066 \\
& $90^{\circ}$ & 0.0002 & 0.0015 & 0.0059 & 0.0075 & 0.0014 & 0.0054 & 0.0069 \\
& $60^{\circ}$ & 0.0004 & 0.0016 & 0.0060 & 0.0075 & 0.0016 & 0.0051 & 0.0067 \\
& $30^{\circ}$ & 0.0039 & 0.0023 & 0.0060 & 0.0084 & 0.0023 & 0.0052 & 0.0075 \\
\hline \hline \multirow{5}{*}{$64 \mathrm{~A} 007.5$} & $2-\mathrm{D}$ & 0.0041 & 0.0056 & 0.0058 & 0.0114 & 0.0054 & 0.0053 & 0.0107 \\
\cline { 2 - 8 } & $90^{\circ}$ (inviscid side walls) & 0.0041 & 0.0058 & 0.0056 & 0.0114 & 0.0057 & 0.0051 & 0.0108 \\
& $90^{\circ}$ & 0.0041 & 0.0052 & 0.0057 & 0.0110 & 0.0051 & 0.0052 & 0.0103 \\
& $60^{\circ}$ & 0.0072 & 0.0069 & 0.0057 & 0.0126 & 0.0067 & 0.0049 & 0.0117 \\
& $30^{\circ}$ & 0.0168 & 0.0110 & 0.0056 & 0.0166 & 0.0103 & 0.0049 & 0.0151 \\
\hline
\end{tabular}

of the shock located at $x / c=0.60$, as seen in Figure 5.5. The reduction of the strut-wall angle to $\phi=60^{\circ}$ is accompanied by a stronger shock. That shock persists over $40 \%$ of the strut span and explains the jump in the drag coefficient. The case with $\phi=30^{\circ}$ has a much stronger shock near the root than the one observed for $\phi=60^{\circ}$. That shock doesn't dissipate quickly away from the wall. The strength of the shock and its extent along the wing span explain the significant drag coefficient calculated for that case. The numerical results show the influence of the strut thickness on the interference drag. For example, for $\phi=30^{\circ}$, the NACA 64A007.5 section produces a drag penalty more than four times higher than the equivalent arrangement for the thinner NACA 64A005 section.

\subsubsection{Navier-Stokes Calculations}

For the viscous calculations, the grids generated are based on a grid point distribution similar to the one used for the inviscid grids. However, the growth rate of the viscous layers in the direction normal to a viscous surface can be controlled through the following equation [101]:

$$
\delta_{i}=\delta_{1}\left[1+r_{1}\left(1+r_{2}\right)^{i-1}\right]^{i-1}
$$

This means that the normal grid spacing of the $i^{t h}$ viscous layer $\delta_{i}$ is a function of the initial spacing at the wall $\delta_{1}$ and the growth rates $r_{1}$ and $r_{2}$. The growth rate $r_{1}$ is set to 0.15 and 0.40 for 2-D and 3-D calculations respectively. For full Navier-Stokes computations like the one at hand, it is recommended to use $r_{2}=0$ [103]. For the computations in the range of 


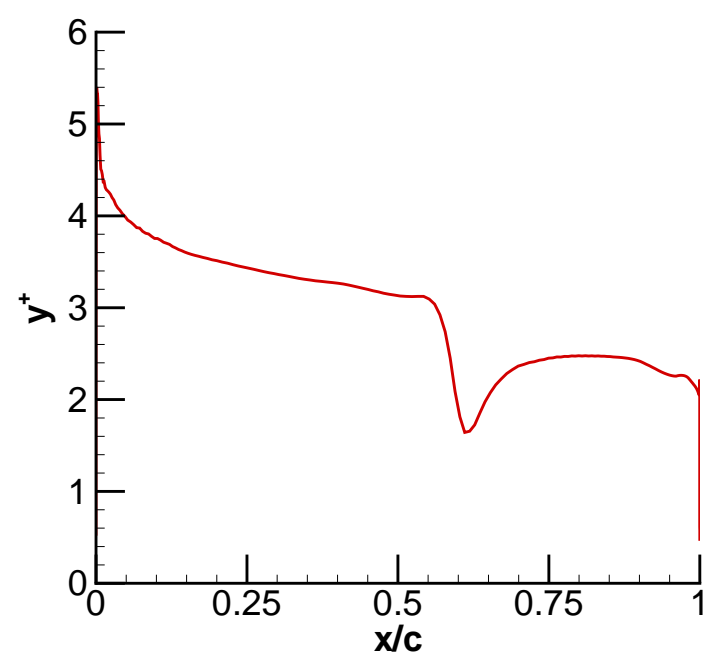

Figure 5.7: Variation of $y^{+}$for the first grid point from the NACA 64A007.5 airfoil surface, $M_{\infty}=0.85, \alpha=0^{\circ}, R e_{c}=10.6 \times 10^{6}$ (Navier-Stokes solution)

$R e_{c}=5.3 \times 10^{6}$ to $10.6 \times 10^{6}$, a grid spacing at the wall $\delta_{1}=1 \times 10^{-5}$ per unit chord allows for at least one point in the laminar sublayer of the turbulent boundary layer. For the NACA $64 \mathrm{~A} 007.5$ airfoil with $R e_{c}=10.6 \times 10^{6}$, the corresponding value of $y^{+}$along the airfoil surface is shown in Figure 5.7. Over the entire range, the first grid point is within the laminar sublayer whose limit is at $y^{+} \approx 8$.

To evaluate the sensitivity of the drag coefficient to these parameters, sample 2-D calculations were performed on the NACA 64A007.5 airfoil section at $\alpha=0^{\circ}$. They revealed that reducing the wall spacing $\delta_{1}$ even by a factor of 10 changes $C_{d}$ by less than $1 \%$, thus confirming the adequate choice of $\delta_{1}=1 \times 10^{-5}$ chord for the initial grid spacing at the wall. On the other hand, decreasing $r_{1}$ from 0.40 to 0.15 increases the predicted $C_{d}$ by less than $2 \%$ at the expense of a grid size that increases by $61 \%$. The cost of using $r_{1}=0.15$ for 3-D computations is too prohibitive in terms of memory requirements. For that reason, $r_{1}=0.40$ was used for all 3-D analyses here.

The characteristics of the viscous grids generated for all strut-wall arrangements are provided in Table 5.3. The number of viscous nodes represent the number of nodes generated by VGRIDns using the advancing-layers methodology [101]. The mesh size is also given for the two-dimensional airfoil sections. The surface grid for the three-dimensional NACA 64A007.5 strut section at $\phi=90^{\circ}$ between parallel inviscid side walls is shown in Figures 5.8 and 5.9. 


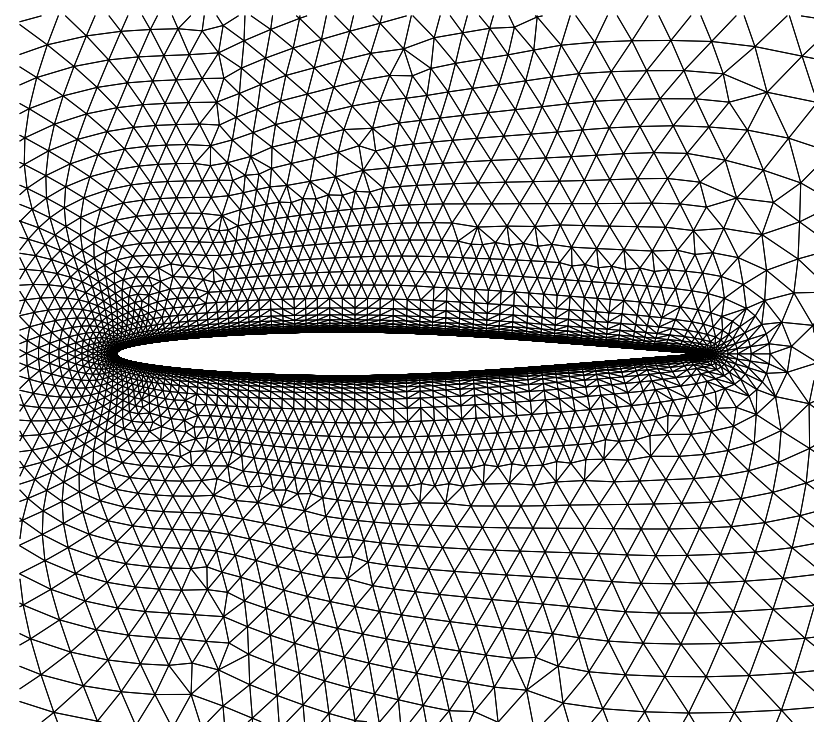

Figure 5.8: Fine viscous surface grid at the end plane of the NACA 64A007.5 strut $-\phi=90^{\circ}$

In Figure 5.9, there is more grid stretching along the span near the symmetry plane than near the side wall. For the configuration between inviscid side walls, constant stretching could have been employed along the entire span since the flow is essentially two-dimensional. But for the case between viscous side walls, spanwise stretching should be avoided near the side wall. Instead of having two different sets of distributed grid sources (one with stretching across the span and the other with stretching limited to the region away from the side wall), the grid sources distributed in the field are identical for both cases. This was done in order to facilitate the grid generation for cases between viscous side walls and inviscid side walls. The only difference is the boundary condition specified on the side wall: no-penetration condition only for inviscid side wall and no-slip condition for viscous side wall. If the no-slip condition is imposed, VGRIDns generates viscous layers on that wall for accurate resolution of the boundary layer.

To assess the quality of the viscous grids generated for the NACA airfoil sections, a grid convergence study was performed for the $7.5 \%$-thick section perpendicular to two viscous side walls. Because of the symmetry of the geometry with respect to the mid span section, only half the configuration was modeled with one of the side walls now being a symmetry plane. Three grids were generated, ranging from coarse to fine in terms of grid resolution. The overall grid characteristics are presented in Table 5.5.

The Navier-Stokes flow solutions were obtained for these configurations for $M_{\infty}=0.85$, 


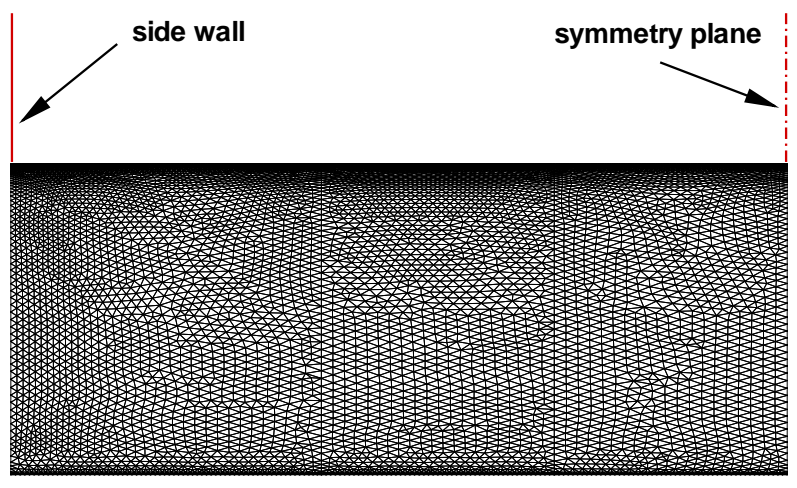

Figure 5.9: Upper surface triangulation of the NACA 64A007.5 strut $-\phi=90^{\circ}$ (inviscid side walls)

Table 5.5: Grid convergence for the NACA 64A007.5 strut - $\phi=90^{\circ}, M_{\infty}=0.85, \alpha=0^{\circ}$, $R e_{c}=5.3 \times 10^{6}$

\begin{tabular}{|c||c|c|c||c|c|c|}
\hline Grid & $\begin{array}{c}\text { Number of } \\
\text { surface nodes }\end{array}$ & $\begin{array}{c}\text { Number of } \\
\text { viscous nodes }\end{array}$ & $\begin{array}{c}\text { Total number } \\
\text { of nodes }\end{array}$ & $C_{D_{p}}$ & $C_{D_{v}}$ & $C_{D_{\text {tot }}}$ \\
\hline Coarse & 10,988 & 177,287 & 205,175 & 0.0059 & 0.0057 & 0.0116 \\
Medium & 15,321 & 244,364 & 299,862 & 0.0055 & 0.0057 & 0.0112 \\
Fine & 25,022 & 408,515 & 529,717 & 0.0052 & 0.0057 & 0.0110 \\
\hline
\end{tabular}




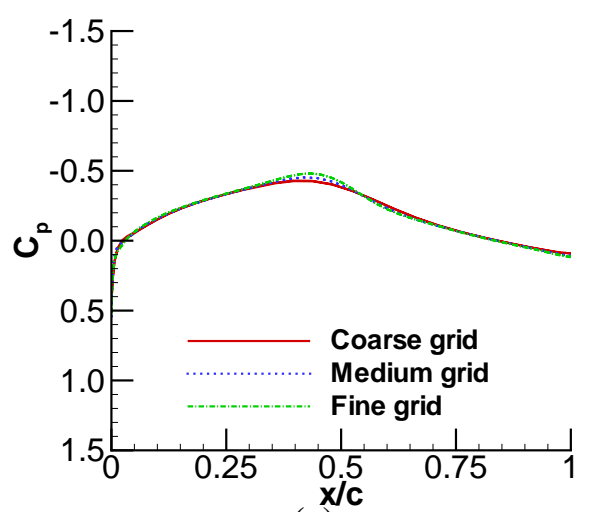

(a)

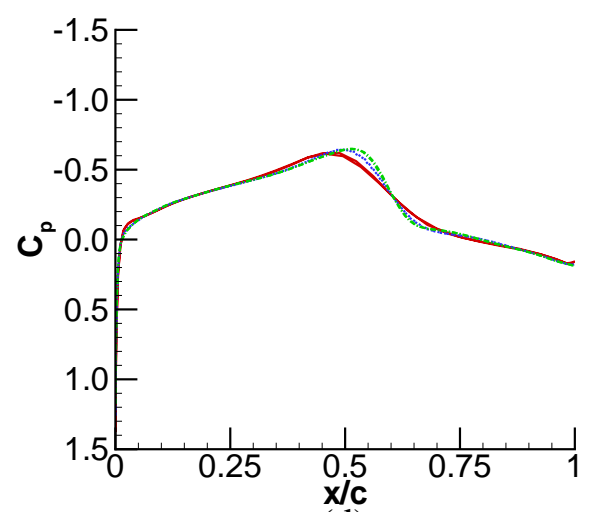

(d)

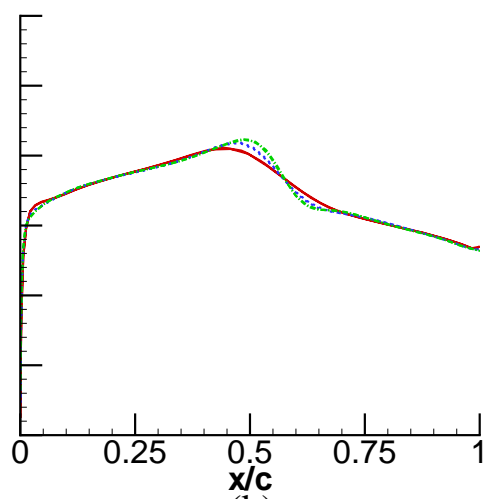

(b)

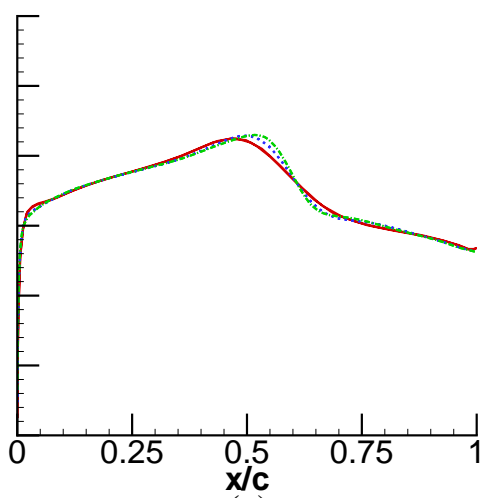

(e)

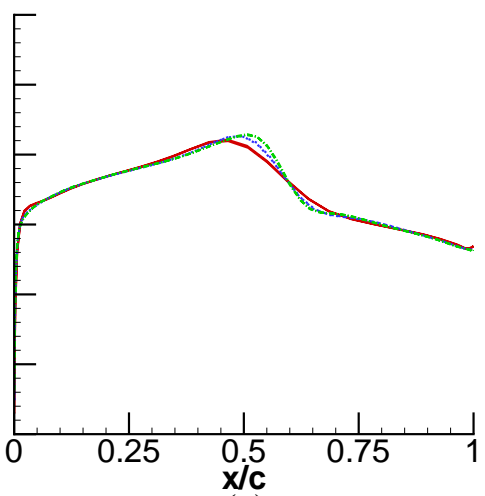

(c)

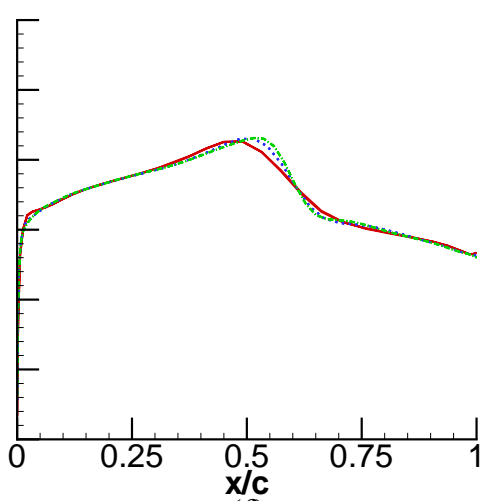

(f)

Figure 5.10: Effects of grid refinement on $C_{p}$ distribution for the NACA 64A007.5 strut $\phi=90^{\circ}, M_{\infty}=0.85, \alpha=0^{\circ}, R e_{c}=5.3 \times 10^{6}$ (Navier-Stokes solution) (a) $\eta=0 \%$ (b) $\eta=10 \%$ (c) $\eta=20 \%$ (d) $\eta=30 \%$ (e) $\eta=40 \%$ (f) $\eta=50 \%$

$\alpha=0^{\circ}$, and $R e_{c}=5.3 \times 10^{6}$. The pressure distributions are provided in Figure 5.10 for several cross sections along the span of the strut. The major difference among these plots is the resolution of the shock at midchord. The finest grid provides the best result for resolving the shock. Another aspect worth mentioning, even if it is barely noticeable, is the presence of a sudden decrease in $C_{p}$ right at the trailing edge of the strut for the coarse grid. This very common phenomenon happens when the grid is not refined enough at the trailing edge thus leading to the formation of skewed cells there. This topic is discussed in detail in Section 6.3.

The magnitude of the lift coefficient is less than $6 \times 10^{-4}$ for all grids although it should be zero. These inaccuracies are due to the grid. The drag coefficients are presented in Table 5.5 and illustrated in Figure 5.11. The pressure drag $C_{D_{p}}$ varies quite significantly with grid refinement because of the poor shock resolution on the coarser grids. Despite that, 


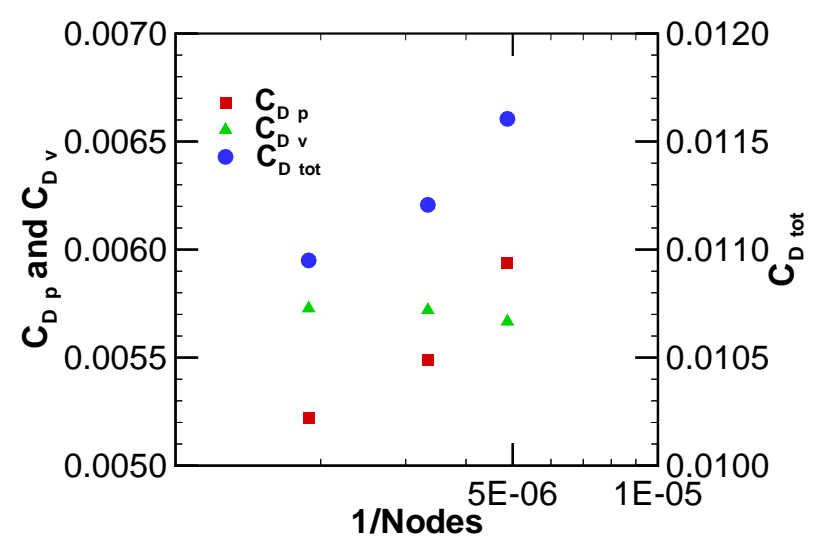

Figure 5.11: Grid convergence study, $M_{\infty}=0.85, \alpha=0^{\circ}, R e_{c}=5.3 \times 10^{6}$ (Navier-Stokes solution) - Pressure and skin friction drag components (left axis) and total drag coefficient (right axis)

the skin friction drag $C_{D_{v}}$ is almost constant for each case, resulting in an overall effect that depends mostly on the pressure drag. When the number of nodes is increased, the total drag coefficient seems to be reaching an asymptotic value. Therefore, the grid point distribution used for the fine mesh presented here was used throughout the viscous analyses shown later.

Richardson extrapolation [115] can be employed to estimate the exact value of the drag coefficient based on the results presented in Table 5.5. The approach is applied in a heuristic way rather than a rigorous one since the unstructured grids are not refined in a consistent fashion. In the viscous layers for instance, all grids use the same growth rates and spacing at the wall. Thus, in the direction normal to the viscous surfaces, all grids have the same grid point distribution. Taking the grid refinement ratio $r$ as the ratio of the total number of nodes on the fine grid to the number of nodes on the medium grid, the exact value of the drag coefficient $C_{D}$ is approximated with the following equation:

$$
C_{D_{\text {exact }}} \approx C_{D_{\text {fine }}}+\frac{C_{D_{\text {fine }}}-C_{D_{\text {medium }}}}{r^{2}-1}
$$

This yields a value of $C_{D_{\text {exact }}}=0.0108$, a difference in drag coefficient of 0.0002 compared to the value obtained on the fine grid. This gives a measure of the discretization error on the fine grid used for the viscous calculations.

Another source of error is the criterion employed to determine the convergence of the CFD solution. The convergence history for the NACA 64A007.5 case with $\phi=30^{\circ}$ is shown in Figure 5.12. The residual decreases four orders of magnitude steadily. The largest 


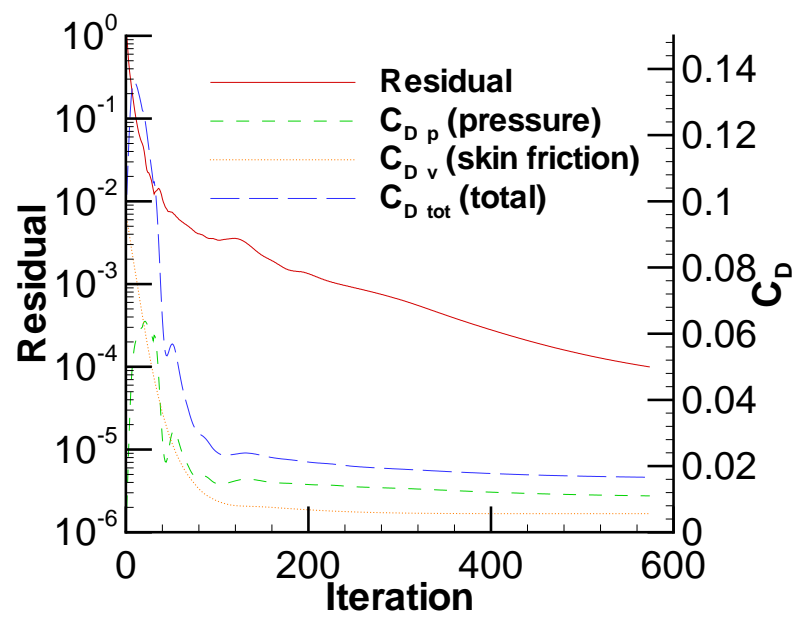

Figure 5.12: Convergence history for the NACA 64A007.5 strut $-\phi=30^{\circ}, M_{\infty}=0.85$, $\alpha=0^{\circ}, R e_{c}=5.3 \times 10^{6}$ (Navier-Stokes solution) - Residual (left axis) and components of the drag coefficient (right axis)

variation of the pressure drag $C_{D_{p}}$ and the skin friction drag $C_{D_{v}}$ occurs during the first 200 iterations. Between iteration 400 and 600, both components of the drag coefficient have reached an asymptotic value. The total drag $C_{D_{t o t}}$ can be considered converged when the solution reaches the point where the residual has been reduced four orders of magnitude, which is a commonly used criterion in the CFD community. This criterion is applied to all the viscous calculations presented in this chapter. Since the other configurations have a convergence history similar to this one, this ensures that the results obtained for each arrangement are comparable with one another.

Because there is little difference between the pressure plots at the two Reynolds numbers chosen for this study, only the pressure distributions for $R e_{c}=5.3 \times 10^{6}$ are presented here. For the NACA 64A005 section results in Figure 5.13, it can be observed that the pressure distribution behaves nicely near the wall compared to the inviscid case. The viscosity dissipates the strong shock at the strut-wall junction very effectively. And, the effect of the wall disappears very quickly as we move away from it. At a station $\eta=30 \%$, the perturbation in the pressure distributions is gone. This is accomplished more quickly than for the inviscid solution. The pressure distribution on the NACA 64A007.5 strut section is depicted in Figure 5.14. As can be seen, there is a more dramatic influence of the wall in the vicinity of the junction. The flow separates at about $x / c=0.65$ on the upper surface of the strut for the $\phi=30^{\circ}$ case. But, from the other cross sections, we can see that the 


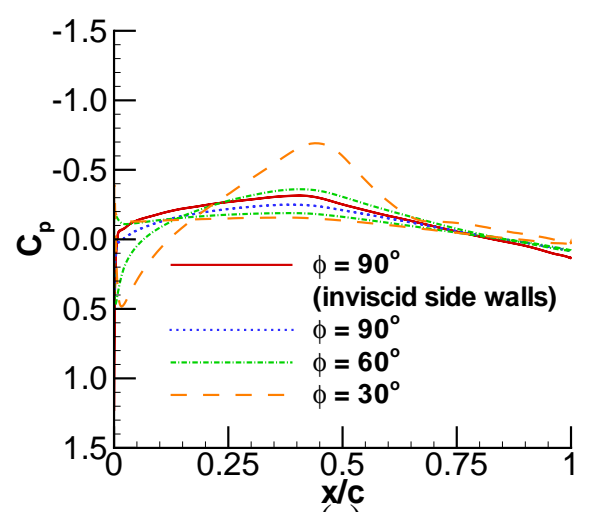

(a)

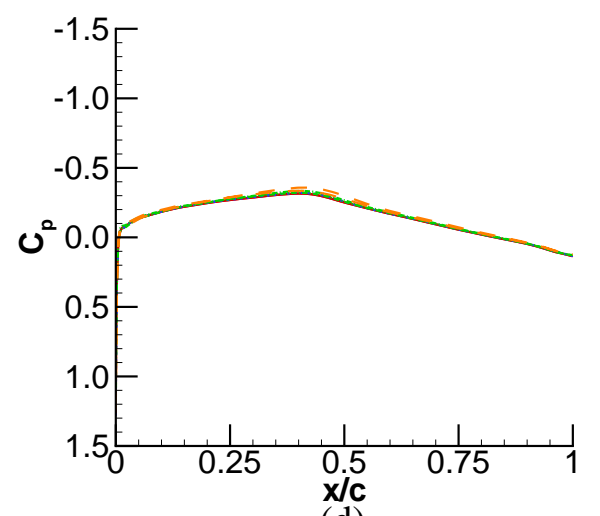

(d)

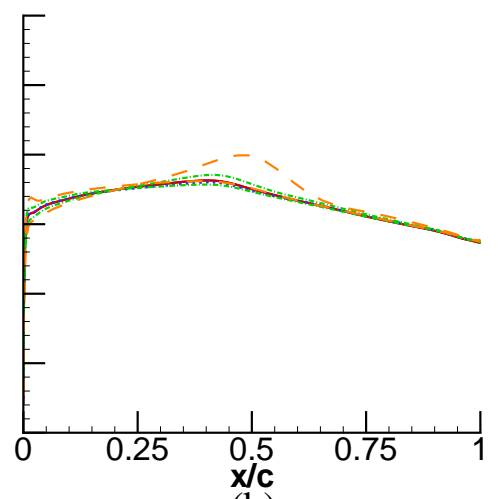

(b)

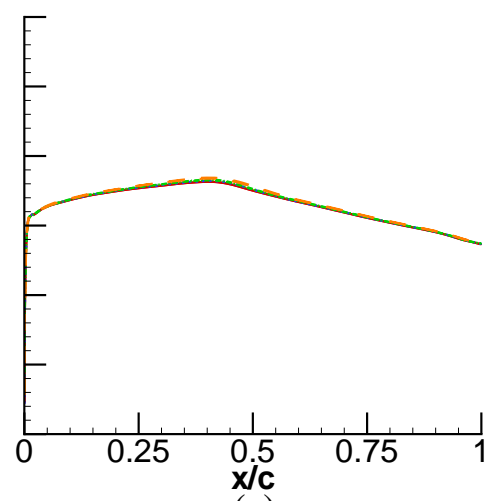

(e)

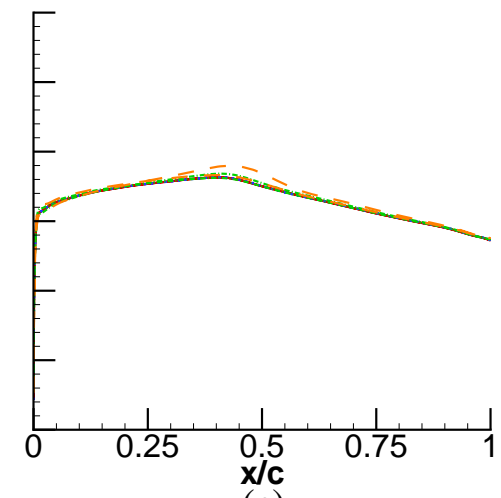

(c)

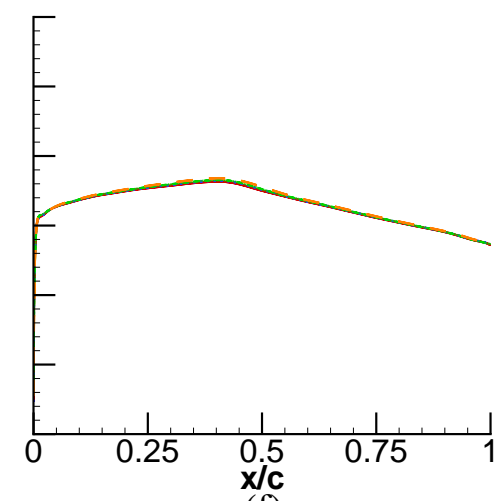

(f)

Figure 5.13: $C_{p}$ distribution for the NACA 64A005 strut, $M_{\infty}=0.85, \alpha=0^{\circ}, R e_{c}=5.3 \times$ $10^{6}$ (Navier-Stokes solution) (a) $\eta=0 \%$ (b) $\eta=10 \%$ (c) $\eta=20 \%$ (d) $\eta=30 \%$ (e) $\eta=40 \%$ (f) $\eta=50 \%$

effect of the wall again dissipates rapidly as the distance along the span increases.

The load distribution on the strut is depicted in Figure 5.15. The lift distribution for the NACA 64A005 strut section is similar for both Reynolds numbers studied. There is an increase in the loading near the root and the tip of the strut as the angle $\phi$ is reduced. However, as opposed to the Euler calculations, there is approximately $50 \%$ of the span that carries negligible load between $\eta=25 \%$ and $75 \%$. Because of its higher thickness, the NACA 64A007.5 strut shows more effect on the load near the side walls. As opposed to the case of the NACA 64A005 strut section, the charts for NACA 64A007.5 are not antisymmetric with respect to mid span for $\phi=30^{\circ}$. As dicussed before, that case has a region of separated flow near the root and the tip. The discrepancy could be explained by the fact that the grid may not be identical near each junction, thus having an impact on the determination of the region of separated flow. Despite that, it should be noted that the lift 


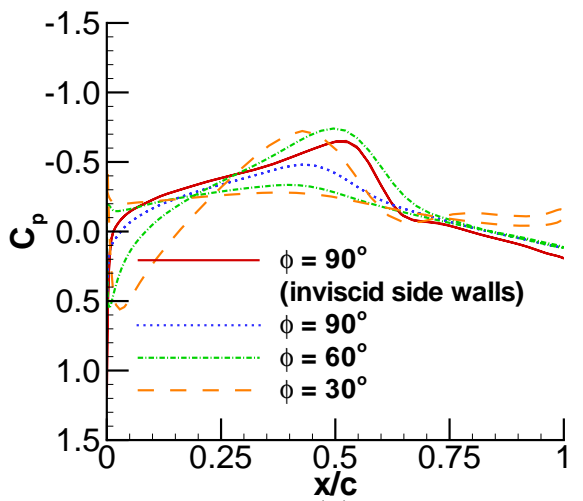

(a)

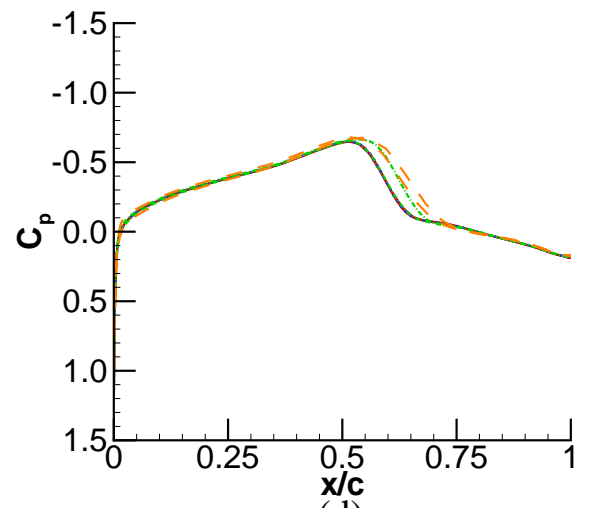

(d)

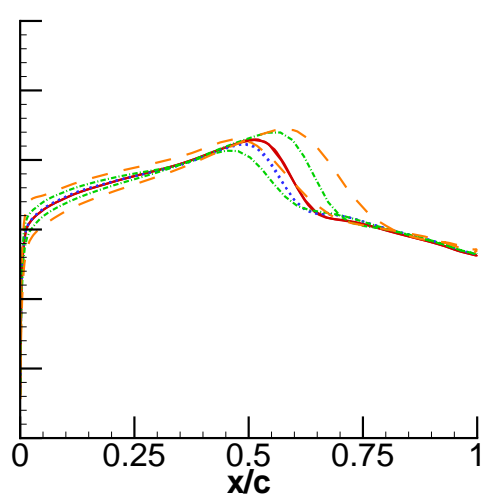

(b)

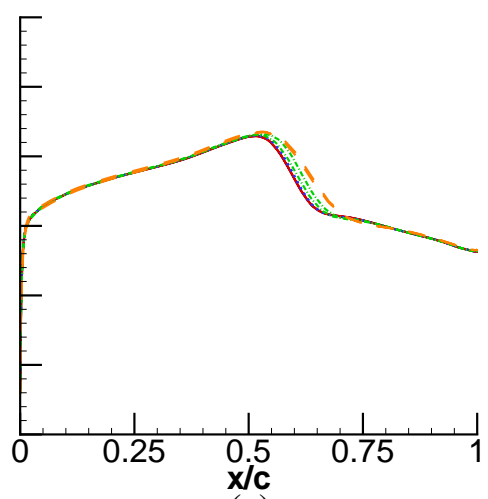

(e)

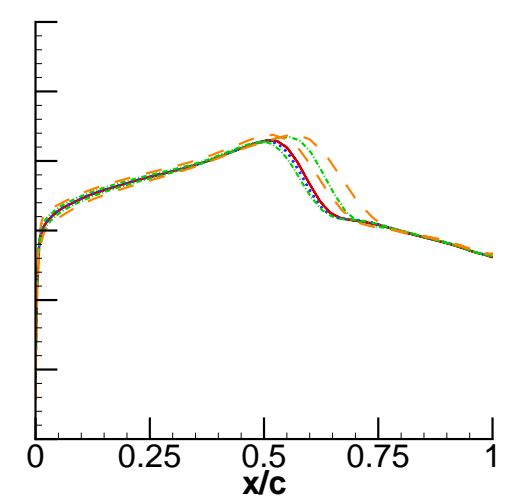

(c)

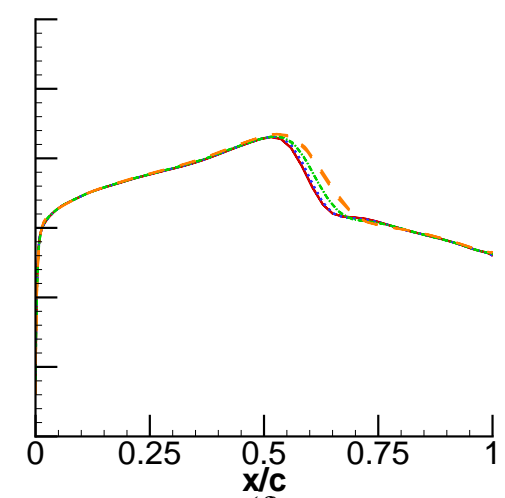

(f)

Figure 5.14: $C_{p}$ distribution for the NACA 64A007.5 strut, $M_{\infty}=0.85, \alpha=0^{\circ}$, $R e_{c}=5.3 \times 10^{6}$ (Navier-Stokes solution) (a) $\eta=0 \%$ (b) $\eta=10 \%$ (c) $\eta=20 \%$ (d) $\eta=30 \%$ (e) $\eta=40 \%$ (f) $\eta=50 \%$ 
coefficient is small for that case. Also, since we consider two junctions instead of just one, this averages the trends in the results.

The lift coefficient is less than 0.005 in magnitude for all the cases analyzed. The drag coefficients for $R e_{c}=5.3 \times 10^{6}$ and $R e_{c}=10.6 \times 10^{6}$ are listed in Table 5.4. For both strut thicknesses, two sets of results for $\phi=90^{\circ}$ are presented, one involving viscous side walls (no-slip condition) and the other one representing the case for inviscid side walls (nopenetration, slip condition). The latter is used as a basis for comparing with an equivalent 2D airfoil calculation done at the same conditions on a very fine grid. According to the results presented here, the calculation performed on a strut between parallel inviscid surfaces agrees well with its 2-D counterpart. The 2-D prediction gives a somewhat lower pressure drag coefficient $C_{D_{p}}$ while providing the solution with a somewhat higher viscous drag component $C_{D_{v}}$. Overall, the effects tend to cancel out to yield a maximum discrepancy of about 0.0001 for the NACA $64 \mathrm{~A} 007.5$ section at $R e_{c}=10.6 \times 10^{6}$.

For the NACA 64A007.5 section at $\phi=90^{\circ}$, the presence of the viscous side walls alleviates the drag penalty obtained for the same strut between inviscid surfaces. This was observed by the shock rendered much weaker in the vicinity of the wall, as shown in Figure 5.14. On the other hand, the NACA 64A005 section doesn't exhibit favorable interference. In fact, the adjacent viscous walls cause the overall drag to increase by a few counts. This interesting phenomenon can be explained by the fact that, as opposed to inviscid Euler calculations which predict a sharp shock provided the grid is fine enough, the Navier-Stokes computations add viscous dissipation that smears the shock. Near the side wall, the strut gets even more relief because the flow is inside the side wall boundary layer. Hence, the shock for the NACA 64A007.5 section almost vanishes, leading to a reduction in the drag compared to the case where inviscid side walls are employed. On the other hand, the NACA 64A005 case doesn't exhibit a shock when it is analyzed between inviscid side walls, and the presence of a viscous side wall doesn't provide a big relief like for the thicker strut.

For the NACA 64A005 strut section, the change of $\phi$ from $90^{\circ}$ to $60^{\circ}$ is accompanied by a negligible increase in drag because the strut section is still shockless. As opposed to the $\phi=60^{\circ}$ case, the arrangement with $\phi=30^{\circ}$ has a shock near the root, causing a net increase in the pressure drag. The drag coefficient for the NACA 64A007.5 strut shows a more rapid increase than the NACA 64A005 section as the strut-wall angle $\phi$ is reduced. When going from $\phi=90^{\circ}$ to $\phi=60^{\circ}$, the shock strength near the side wall is amplified 


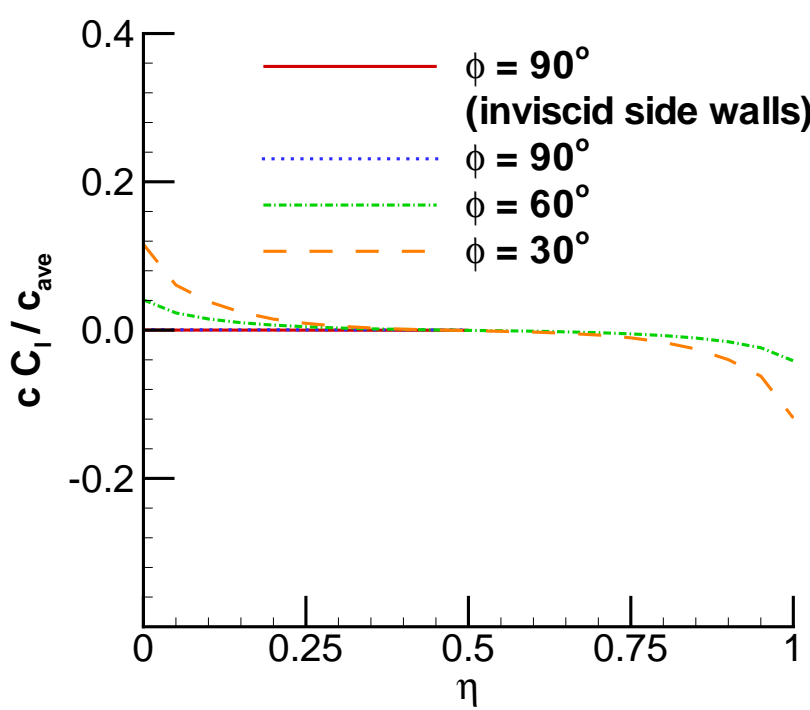

(a)

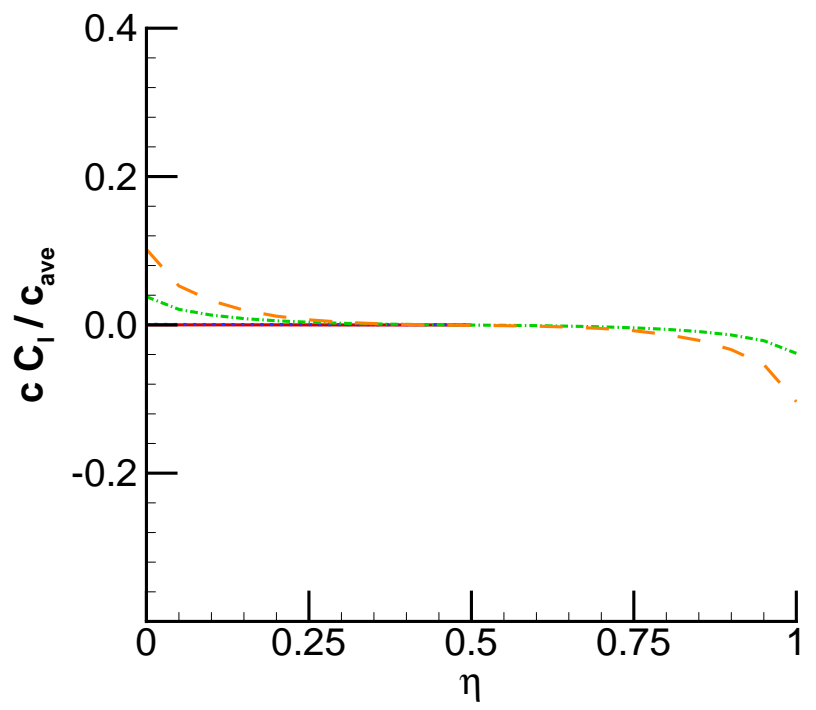

(c)

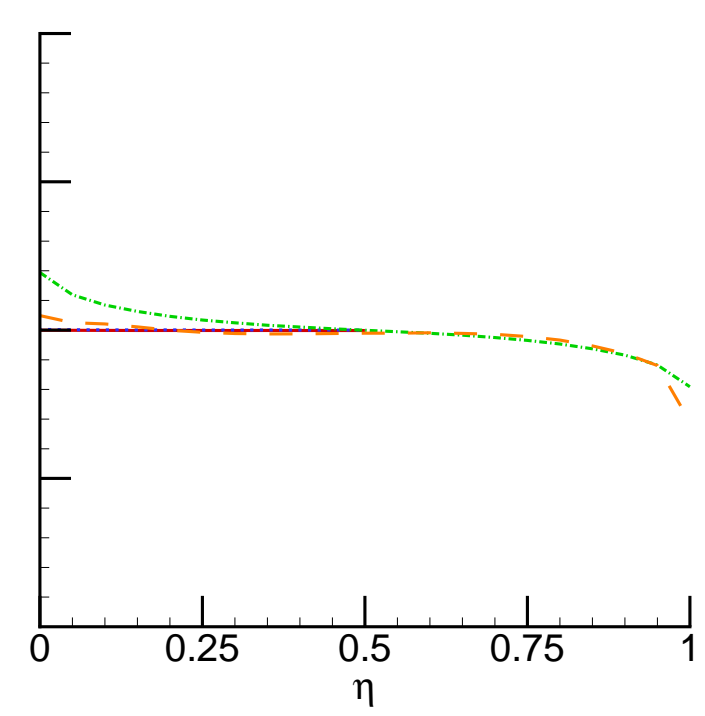

(b)

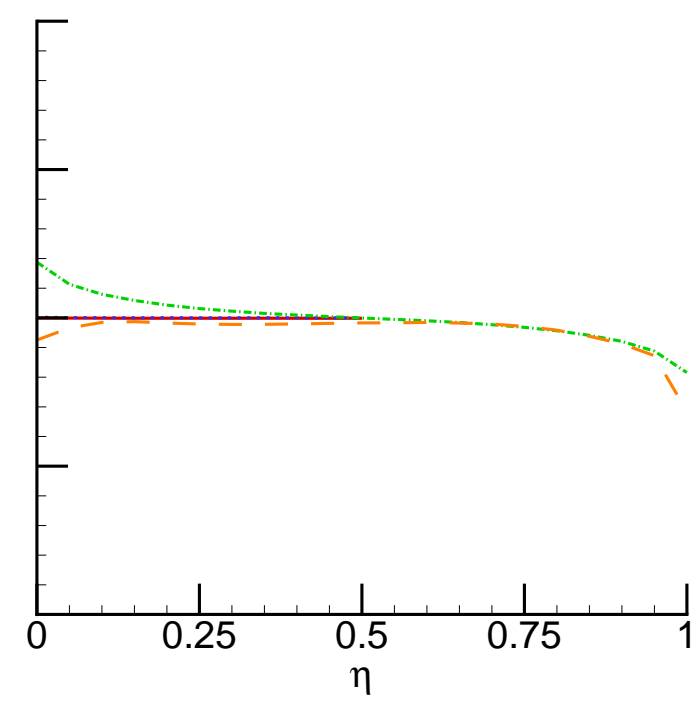

(d)

Figure 5.15: Spanload distribution, $M_{\infty}=0.85, \alpha=0^{\circ}$ (Navier-Stokes solution) (a) NACA 64A005 with $R e_{c}=5.3 \times 10^{6}$ (b) NACA 64A007.5 with $R e_{c}=5.3 \times 10^{6}$ (c) NACA 64A005 with $R e_{c}=10.6 \times 10^{6}$ (d) NACA 64A007.5 with $R e_{c}=10.6 \times 10^{6}$ 
and leads to an increase in the pressure drag coefficient. The increase in the drag coefficient for $\phi=30^{\circ}$ can be explained by the strong shock and the large region of separated flow in the vicinity of the strut-wall junction. This is reflected in the pressure drag coefficient. Despite the presence of a region of separated flow near the intersection of the strut with the wall for $\phi=30^{\circ}$, the pressure drag coefficient and even the overall drag coefficient are less than the drag coefficient predicted by the Euler computations in Table 5.4. This confirms that the viscous effects can contribute favorably to the drag of the strut by alleviating the strength of the shock near the wall and by dissipating its effect rapidly in the flowfield.

All the results presented in Table 5.4, can be interpreted in the form described by Eq. 5.10. For the Euler calculations, the case for which $\phi=90^{\circ}$ was used as the reference $C_{D_{S}}$. For the Navier-Stokes analyses, the reference value for $C_{D_{S}}$ is chosen as the value calculated for $\phi=90^{\circ}$ between inviscid side walls. The results are listed in Table 5.6. The interference drag numbers highlight how dramatic the increase in drag is if the viscous effects are not considered. The NACA 64A007.5 airfoil with $\phi=90^{\circ}$ exhibits favorable interference as the negative interference drag numbers suggest. One thing to note is that no fairing or contouring was applied near the junction of the strut with the wall. In the subsonic flow range, Hoerner [24] observed that a junction with fairing has at least 10 times less interference drag than an unfaired one. Thus, the numbers presented in Table 5.6 could be reduced significantly with the use of fairings.

The statistical software JMP [116] was employed to fit a quadratic response surface to the interference drag coefficient using a standard least-squares procedure. Only the NavierStokes results were considered in this analysis. The equation can be included in MDO studies to account for the interference drag penalty of wing-strut junctions or other related configurations. The response surface can be written as:

$$
\begin{aligned}
C_{D_{\text {interf }}} & =0.1112-0.2572 \sin \phi+3.440(t / c)-0.02097 \log _{10} R e_{c}+0.09009 \sin ^{2} \phi \\
& -2.549(t / c) \sin \phi+0.03010 \log _{10} R e_{c} \sin \phi-0.1462(t / c) \log _{10} R e_{c}
\end{aligned}
$$

where $t / c$ is 0.05 for a $5 \%$-thick airfoil. The response surface has a coefficient of determination $R^{2}=0.9987$ with a root mean square (RMS) error of 0.001177 . The curve is shown in Figure 5.16 for $R e_{c}=5.3 \times 10^{6}$.

The comparison of the original interference drag data from Table 5.6 with the prediction of the response surface is shown in Figure 5.17. The response surface is in good agreement with the data over the range of $\phi$ and correctly predicts favorable interference for the 


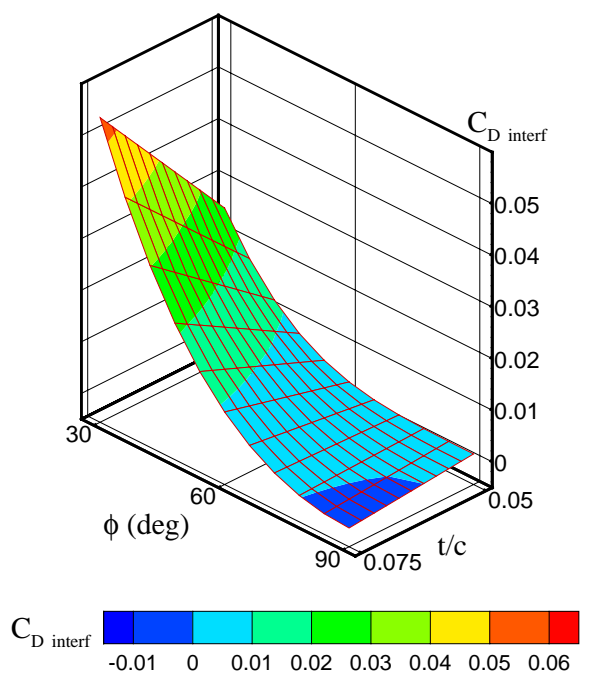

Figure 5.16: Response surface with contours of $C_{D_{\text {interf }}}$ for $R e_{c}=5.3 \times 10^{6}$

Table 5.6: Interference drag coefficients

\begin{tabular}{|c|c||c|c|c|}
\hline NACA & \multicolumn{1}{|c||}{$\phi$} & \multicolumn{3}{c|}{$C_{D_{\text {interf }}}=\frac{D_{\text {interf }}}{q_{\infty o c} c^{2}}$} \\
\cline { 3 - 5 } airfoil & & Euler & $R e_{c}=5.3 \times 10^{6}$ & $R e_{c}=10.6 \times 10^{6}$ \\
\hline & $90^{\circ}$ & 0.0000 & 0.0006 & 0.0006 \\
$64 \mathrm{~A} 005$ & $60^{\circ}$ & 0.0007 & 0.0038 & 0.0028 \\
& $30^{\circ}$ & 0.0188 & 0.0238 & 0.0207 \\
\hline \hline & $90^{\circ}$ & 0.0000 & -0.0010 & -0.0011 \\
$64 \mathrm{~A} 007.5$ & $60^{\circ}$ & 0.0105 & 0.0080 & 0.0067 \\
& $30^{\circ}$ & 0.0739 & 0.0547 & 0.0487 \\
\hline
\end{tabular}

NACA 64A007.5 section with $\phi=90^{\circ}$. In general, the fact that the highest Reynolds number produces less interference drag is captured correctly except for the NACA 64A005 section with $70^{\circ}<\phi \leq 90^{\circ}$. Over that range, the curves of the response surface cross each other. However, the maximum difference between them is small.

\subsection{Flowfield Details}

Pressure contour plots and streamtraces will now be used to better understand the behavior of the flow for the strut-wall configuration. The NACA 64A005 strut section will be shown first, and that will be followed by the presentation of the NACA 64A007.5 case. A discussion 


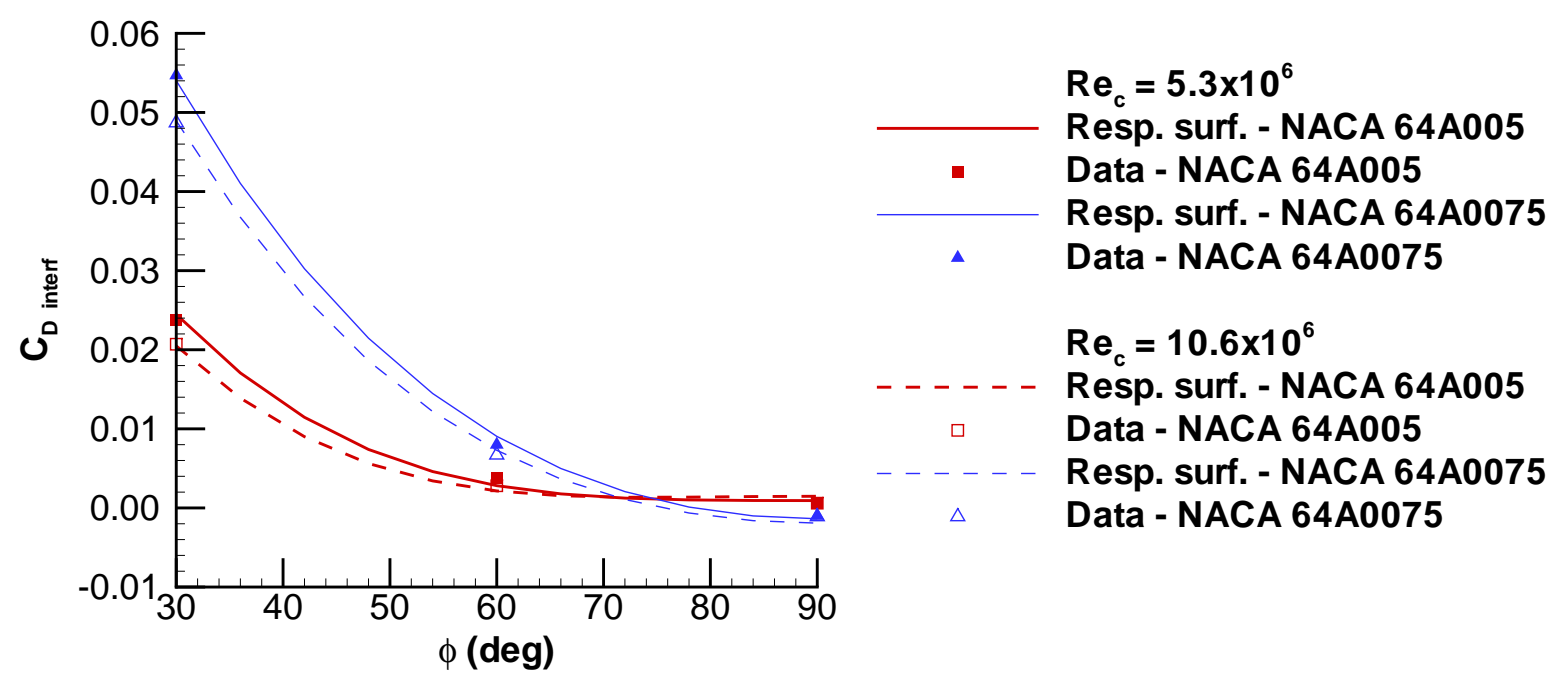

Figure 5.17: Comparison of the response surface with the CFD data for $M_{\infty}=0.85$, $\alpha=0^{\circ}$, and $R e_{c}=5.3 \times 10^{6}$ and $10.6 \times 10^{6}$

will then focus on the two-dimensionality of the flow at the mid span of the strut for both strut sections.

\subsubsection{Flowfield Around the NACA 64A005 Strut Section}

The contours for the NACA 64A005 strut section are shown in Figure 5.18. The figure depicts the Euler and Navier-Stokes solutions for the flowfield a distance $1 \times 10^{-5}$ chord $(\eta=0.0002 \%)$ from the side wall. The flow is from left to right and the strut intersects the plane of the page coming down from above.

Let us consider the Euler solutions first. For $\phi=90^{\circ}$, the pressure contours are limited to a region close to the strut section. The contours are symmetric as could be expected from the symmetry of the geometry analyzed. The stagnation point is located right at the leading edge of the strut. Upstream of that point, the fluid is decelerated as shown by the contours of increasing pressure in the vicinity of the leading edge. The airfoil section employed for the strut is thin and no shock wave is present on the surface. Fluid particles follow the strut surface and leave smoothly at the trailing edge.

When the strut-wall angle is reduced from $\phi=90^{\circ}$ to $60^{\circ}$, the region between the strut upper surface and the nearby wall is narrow and forms a channel that accelerates the flow. 


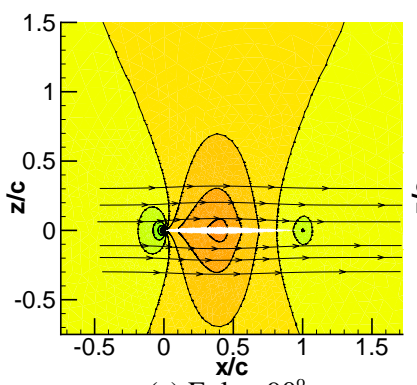

(a) Euler, $90^{\circ}$

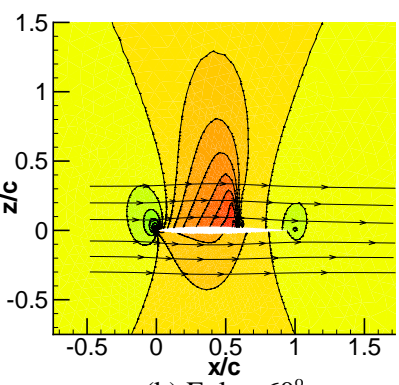

(b) Euler, $60^{\circ}$

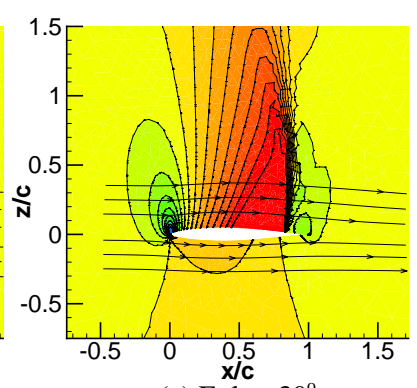

(c) Euler, $30^{\circ}$

$\mathrm{p} / \mathrm{p}_{\infty}: \quad 0.600 .650 .700 .750 .800 .850 .900 .951 .001 .051 .101 .151 .201 .251 .301 .351 .40$

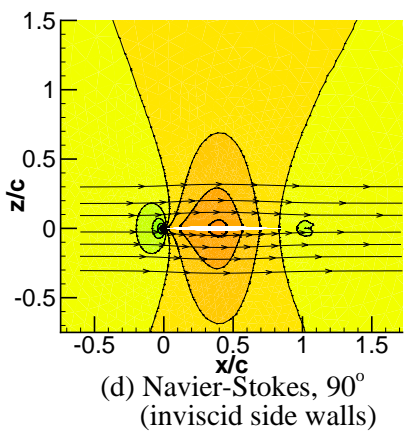

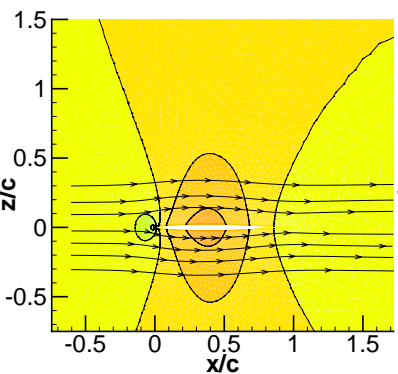

(e) Navier-Stokes, $90^{\circ}$

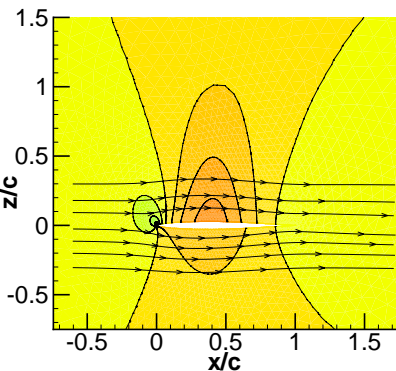

(f) Navier-Stokes, $60^{\circ}$

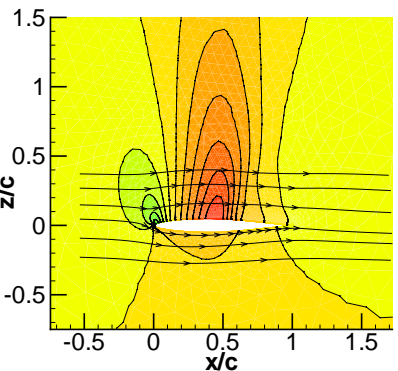

(g) Navier-Stokes, $30^{\circ}$

Figure 5.18: Pressure contours with streamtraces at $\eta=0.0002 \%$ for NACA 64A005, $M_{\infty}=0.85, \alpha=0^{\circ}, R e_{c}=5.3 \times 10^{6}$ - Euler solution: (a) $\phi=90^{\circ}$ (b) $\phi=60^{\circ}$ (c) $\phi=30^{\circ}-$ Navier-Stokes solution: (d) $\phi=90^{\circ}$ (inviscid side walls) (e) $\phi=90^{\circ}$ (f) $\phi=60^{\circ}$ (g) $\phi=30^{\circ}$ 
The fluid particles upstream of the strut come to rest at the stagnation point which is located a short distance downstream of the leading edge on the strut upper surface. The acceleration of the particles on the upper surface leads to the formation of a shock at $x / c=0.60$. On the lower surface, however, the flow is almost undisturbed by the presence of the strut.

Further reduction of the strut-wall angle to $\phi=30^{\circ}$ produces even more effect in the flowfield. The pressure gradient upstream of the strut leading edge extends over a wider region than for the cases previously discussed. The stagnation point also moves downstream on the upper surface. The fluid particles are accelerated quite rapidly on the strut upper surface due to the constrained flow channel. A very strong shock is then formed close to the trailing edge. It is interesting to note how far in the flowfield the disturbances due to the strut propagate. The lower surface again shows almost no sign of the presence of the strut.

Consider now the viscous Navier-Stokes results for $R e_{c}=5.3 \times 10^{6}$. The case with the strut perpendicular to the inviscid side walls shows pressure contours and streamtraces that are very similar to the Euler results for the $\phi=90^{\circ}$ case. After decelerating upstream of the strut, the fluid particles are accelerated very slightly along the strut upper and lower surfaces. Note also the symmetry in the pressure contours and the stagnation point right at the strut leading edge.

If we impose the no-slip condition on the side walls, the side wall boundary layer that is allowed to develop blends away some of the pressure variations encountered for the case between inviscid side walls. The deceleration of the particles upstream of the strut leading edge is not as important because the flow is within the wall boundary layer and is, therefore, slow. The particles accelerate slightly as they reach midchord. They go back to freestream conditions smoothly after.

If we change the strut-wall angle $\phi$ to $60^{\circ}$, the presence of the nearby side wall produces some effect, although not as dramatic as for the inviscid calculations for the same case. The particles do accelerate as they follow the strut surface, but not enough to lead to the formation of a shock. Instead, the particles slowly return to freestream conditions. The viscous forces are shown to have a beneficial effect on the flow because, compared to the inviscid results for $\phi=60^{\circ}$, the pressure contours are attenuated enough to get rid of the shock completely.

Finally, reducing the strut-wall angle to $\phi=30^{\circ}$ is accompanied by more disruption of the flowfield. There is no separation, however, because the strut is thin compared to the 
NACA 64A007.5 section. But, we can clearly see the displacement of the stagnation point downstream of the leading edge. We barely see any change in velocity on the strut lower surface. The upper surface shows contours that extend far above the strut. The viscosity alleviates some of those disturbances, but they propagate a significant distance from the strut anyway. The velocity of the particles following the upper surface changes quite rapidly as the short distance separating the pressure contours shows. The fluid reaches its maximum velocity at mid chord before decelerating to the freestream conditions.

We will now consider the flowfield a distance equivalent to 0.50 chord $(\eta=10 \%)$ away from the side wall. The cross sections are represented in Figure 5.19. We will compare the results with the ones depicted in Figure 5.18 for $\eta=0.0002 \%$.

For the Euler computations, the case where $\phi=90^{\circ}$ is no different than the same case at the $\eta=0.0002 \%$ cross section. This is what should be expected since the flow everywhere along the strut is two-dimensional. Reducing the strut-wall angle to $\phi=60^{\circ}$ creates more disruption of the flowfield. However, the strength of the shock on the strut is weakened due to the increased distance from the side wall. This is because the flow is not constrained by the side wall anymore. We can also notice the fact that the pressure contours in the vicinity of the leading and trailing edges tend to be symmetric with respect to the chord of the airfoil. On the strut lower surface, we can see more variation in the pressure distribution because the flow becomes closer to two dimensional. The same conclusions can be drawn for the $\phi=30^{\circ}$ case. In the vicinity of the wall, the shock was very strong and the pressure contours extended far in the flowfield. As the distance away from the wall is increased, the shock becomes weaker on the upper surface while more disturbances emanate from the lower surface. Near the leading and trailing edges, the pressure contours are close to being symmetric.

If we now look closely at the Navier-Stokes solutions, we can see the consequences of increasing the distance with the side wall. As expected, the case of the strut perpendicular to two inviscid side walls is identical to the cross section made at $\eta=0.0002 \%$. For $\phi=90^{\circ}$ between viscous walls, the effect of the neighboring wall has dissipated and the pressure distribution in the field looks more like the case between inviscid side walls. There is a larger region of high speed flow on the upper and lower strut surfaces than for $\eta=0.0002 \%$. The disturbances around the strut are also more significant. When the strut-wall angle is further reduced to $\phi=60^{\circ}$ and $\phi=30^{\circ}$, the flowfield shows a substantial reduction of the pressure gradient on the strut upper surface. On the lower surface, the extent of the pressure 


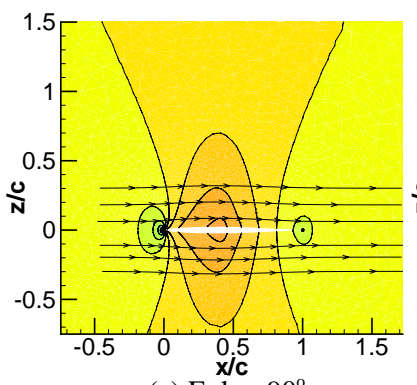

(a) Euler, $90^{\circ}$

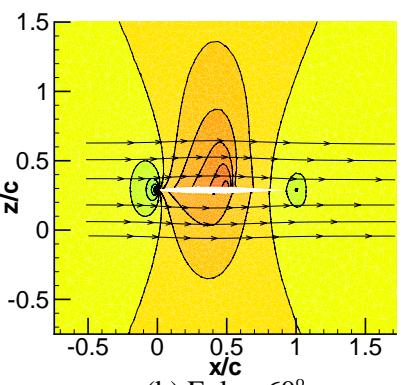

(b) Euler, $60^{\circ}$

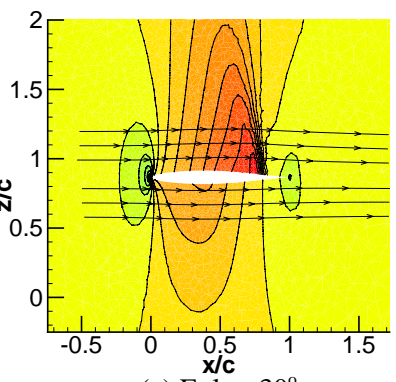

(c) Euler, $30^{\circ}$

$\mathrm{p} / \mathrm{p}_{\infty}: \quad 0.600 .650 .700 .750 .800 .850 .900 .951 .001 .051 .101 .151 .201 .251 .301 .351 .40$

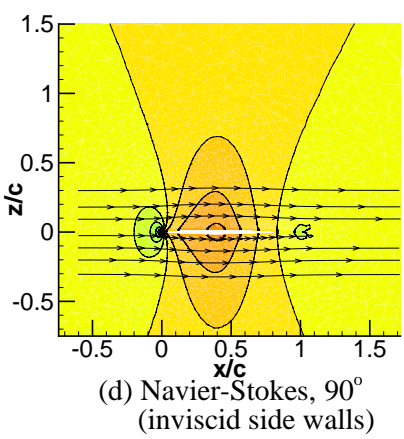

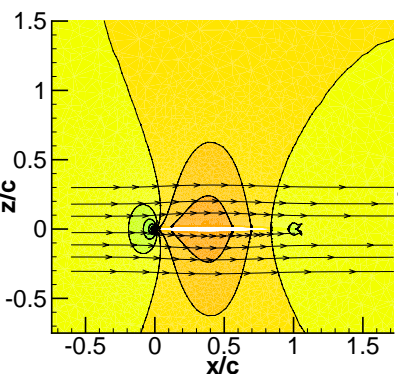

(e) Navier-Stokes, $90^{\circ}$

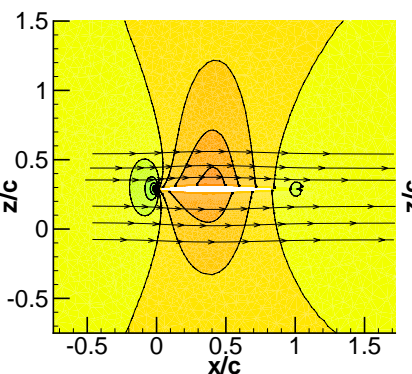

(f) Navier-Stokes, $60^{\circ}$

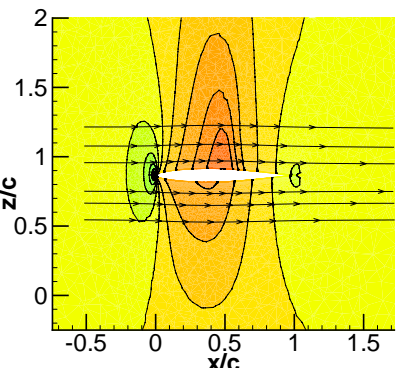

(g) Navier-Stokes, $30^{\circ}$

Figure 5.19: Pressure contours with streamtraces at $\eta=10 \%$ for NACA 64A005, $M_{\infty}=0.85, \alpha=0^{\circ}, R e_{c}=5.3 \times 10^{6}$ - Euler solution: (a) $\phi=90^{\circ}$ (b) $\phi=60^{\circ}$ (c) $\phi=30^{\circ}-$ Navier-Stokes solution: (d) $\phi=90^{\circ}$ (inviscid side walls) (e) $\phi=90^{\circ}$ (f) $\phi=60^{\circ}$ (g) $\phi=30^{\circ}$

contours is greater. It can also be noted that the flow near the leading and trailing edges is symmetric with respect to the strut chord. The flow slowly returns to a two-dimensional state.

\subsubsection{Flowfield Around the NACA 64A007.5 Strut Section}

We will now discuss the case of the NACA 64A007.5 section a distance of $1 \times 10^{-5}$ chord $(\eta=0.0002 \%)$ from the side wall. The pressure contours and streamtraces are depicted in Figure 5.20. The Euler results are presented first. For $\phi=90^{\circ}$, the symmetric strut section creates a symmetry in the pressure contours as well. The stagnation point is located at the leading edge of the strut. The particles decelerate from the freestream velocity when they get in the region near the leading edge, as shown by the contours of increasing pressure. They accelerate along the surface past the sonic conditions, accompanied by a decrease in 


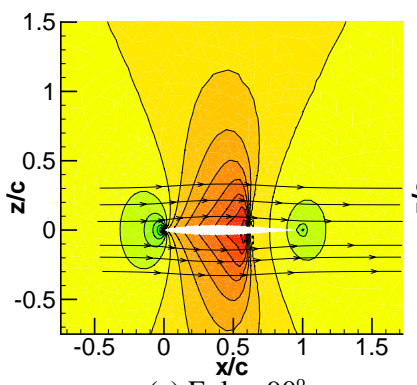

(a) Euler, $90^{\circ}$

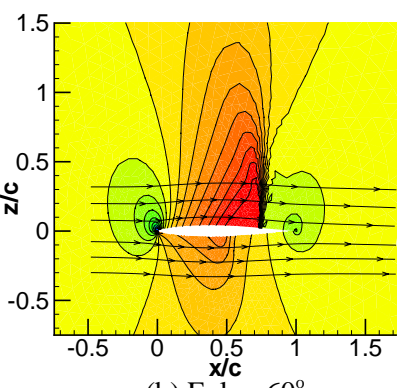

(b) Euler, $60^{\circ}$

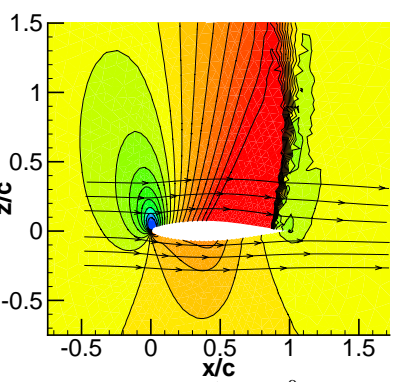

(c) Euler, $30^{\circ}$

$\mathrm{p} / \mathrm{p}_{\infty}: \quad 0.600 .650 .700 .750 .800 .850 .900 .951 .001 .051 .101 .151 .201 .251 .301 .351 .40$

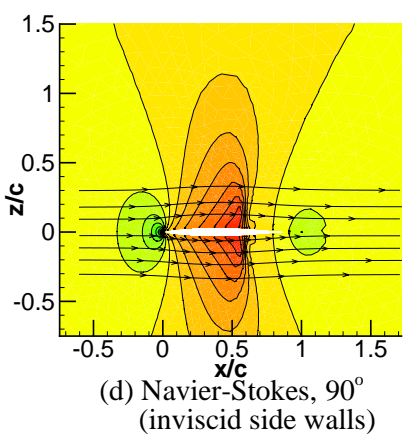

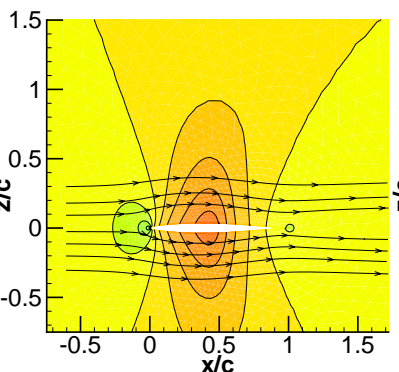

(e) Navier-Stokes, $90^{\circ}$

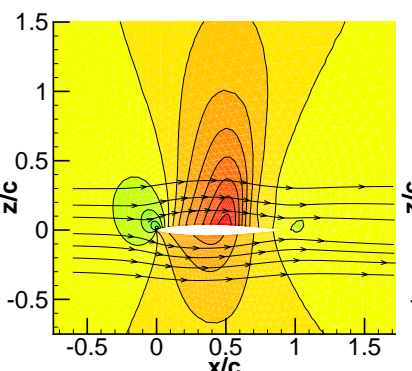

(f) Navier-Stokes, $60^{\circ}$

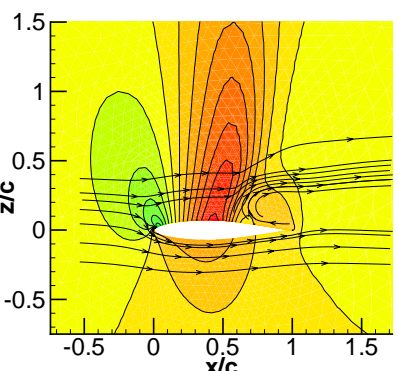

(g) Navier-Stokes, $30^{\circ}$

Figure 5.20: Pressure contours with streamtraces at $\eta=0.0002 \%$ for NACA 64A007.5, $M_{\infty}=0.85, \alpha=0^{\circ}, R e_{c}=5.3 \times 10^{6}-$ Euler solution: (a) $\phi=90^{\circ}$ (b) $\phi=60^{\circ}$ (c) $\phi=30^{\circ}-$ Navier-Stokes solution: (d) $\phi=90^{\circ}$ (inviscid side walls) (e) $\phi=90^{\circ}$ (f) $\phi=60^{\circ}$ (g) $\phi=30^{\circ}$

the pressure. At $x / c=0.60$, the pressure increases sharply as a consequence of the presence of a shock wave at that location. It then returns to its freestream value.

The $\phi=60^{\circ}$ case shows the channel effect as the strut is tilted towards the side wall. The upper surface of the strut is located in that region. The stagnation point moves a short distance away from the leading edge on the upper surface. It should also be noted that the pressure contours upstream of the strut leading edge extend farther away from it than for the $\phi=90^{\circ}$ case. On the upper surface, the particles are accelerated quickly as a result of the curvature of the strut surface and the proximity of the side wall. This can be seen from the pressure contours that get closer to each other in the region between the leading edge and mid chord. There is a large region of supersonic flow on the strut that culminates with a strong shock at $x / c=0.75$. The flow slowly returns to the freestream conditions some distance downstream of the trailing edge. Overall, the presence of a tilted strut produces a lot of disturbance of the flowfield in the channel region. This is depicted by the pressure 
contours that extend in the flowfield a large distance above the strut. On the other hand, in the vicinity of the lower surface, the effect of the wall and the strut have a weaker effect in the flowfield than for $\phi=90^{\circ}$. The particles accelerate at a slow pace on the lower surface and return back smoothly to the freestream conditions.

The configuration with $\phi=30^{\circ}$ amplifies all the trends already highlighted for $\phi=60^{\circ}$. The stagnation point moves downstream on the strut upper surface. The side wall is much closer to the strut upper surface, thus the flow accelerates dramatically as depicted by the rapid variation in the pressure contours. The high-speed flow region ends at $x / c=0.90$ as the pressure lines collapse onto each other to form a very strong shock. The flow goes from a very low pressure back to almost the freestream condition over a very short distance. This produces a severe perturbation of the entire flowfield more than 1.5 chord above the strut. Upstream of the leading edge, the contours go beyond what the $\phi=60^{\circ}$ case showed. This is also due to the fact that the cross sections presented here are made parallel to the side walls, not perpendicular to the strut planform. Care should, therefore, be taken in interpreting distances on these contour plots. On the lower surface of the strut, the contours are considerably weaker than for $\phi=90^{\circ}$ and $\phi=60^{\circ}$ as the flow acceleration and deceleration back to freestream conditions are very smooth.

The Navier-Stokes results are shown for all angles, including the case where $\phi=90^{\circ}$ with inviscid side walls. Since that case is two-dimensional by nature, it allows a preliminary interpretation of the addition of viscous effects to the flow. The pressure contours are symmetric on both sides of the strut with the stagnation point located at the leading edge of the strut. The flow decelerates from the freestream conditions as it gets in the vicinity of the leading edge. The particles accelerate as they follow the strut surface until there is a sudden jump in pressure due to the presence of a shock at $x / c=0.60$. The shock is smeared due to the viscous forces acting on the flow. This is confirmed by the fact that there is some distance between the pressure contours in the region of the shock. It is a weaker shock than the one obtained from the Euler calculation on the same configuration. The flow slows down as it moves toward the trailing edge.

The contour plots for the cases remaining to be discussed all make use of results employing the no-slip condition on the side walls as opposed to the case just presented. The cross sections are located within the side wall boundary layer. The $\phi=90^{\circ}$ case exhibits symmetry in its pressure contours. As shown by the pressure contours confined to a small region upstream of the strut leading edge, the deceleration of the particles is small as they 
get to the stagnation point because they are within the side wall boundary layer. After reaching that point, they accelerate and then decelerate slowly towards the trailing edge. The variations in the flowfield are small because of the presence of the viscous side wall. This effect is beneficial because the shock displayed by the $\phi=90^{\circ}$ case between inviscid side walls disappeared completely. The extent of the pressure contours in the flowfield is also made weaker by the viscous forces of the side wall boundary layer.

For $\phi=60^{\circ}$, the flow decelerates upstream of the strut and reaches the stagnation point a short distance from the leading edge along the upper surface. In the channel region, the fluid particles' acceleration is accompanied by a drop in the pressure. Towards the trailing edge, the flow goes through a shock, but the adverse pressure gradient is not strong enough to cause the flow to separate. Compared to the Euler solution for the same configuration, the Navier-Stokes results show a shock made much weaker by the action of the viscous forces. The shock is located a significant distance upstream of the position predicted by the Euler solution. And, because of the dissipative mechanism of the viscous forces, the pressure contours die out more quickly away from the strut. The strut doesn't perturb the flowfield as strongly as anticipated for that reason. On the lower surface of the strut, the variations of the velocity are very small and the absence of a shock there allows for a smooth pressure distribution. The streamtraces show more curvature around the strut compared to their Euler counterparts. The boundary layers that develop on the strut and the adjacent side wall combine to deflect the particles away from the low speed flow regions adjacent to the strut surface.

The configuration for $\phi=30^{\circ}$ exhibits the most interesting features because of the much stronger interactions in the channel formed by the strut and the adjacent side wall. The fluid particles coming from upstream are deviated sharply away from the channel region as they decelerate towards the strut. This is due to the presence of the boundary layer that develops on the strut and the side wall. As mentioned before, the reduced space between the strut and the wall produces a rapid increase in the velocity of the fluid particles that ends with a shock standing at $x / c=0.60$. That smeared shock is weaker and upstream of the one predicted by the Euler solution due to the dissipative viscous effects. However, as a result of the severe adverse pressure gradient produced by the shock, the flow separates downstream. The streamtraces show a large region of recirculating flow in the vicinity of the strut trailing edge. The fluid particles are deviated away from that vortex. The lower surface of the strut shows small variations in the pressure. Compared to the Euler solution, 


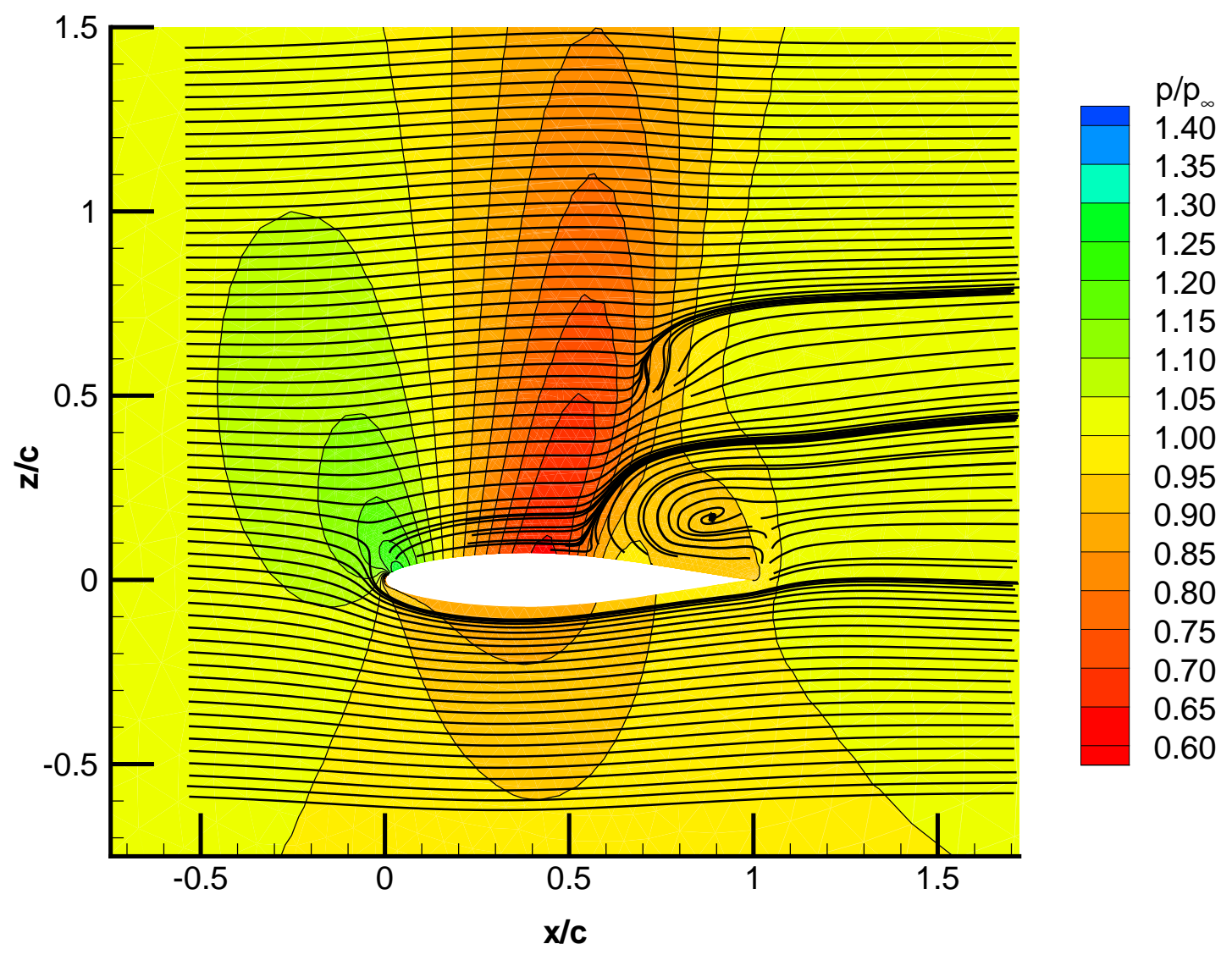

Figure 5.21: Recirculation vortex at $\eta=0.0002 \%$ for NACA 64A007.5 with $\phi=30^{\circ}$, $M_{\infty}=0.85, \alpha=0^{\circ}, R e_{c}=5.3 \times 10^{6}$ (Navier-Stokes solution)

the pressure contours don't extend very far away from the strut. The viscous mechanisms dampen the perturbations induced by the presence of the strut.

The streamlines and Mach number contours for that case are shown in greater detail in Figure 5.21. The recirculation region covers half of the airfoil upper surface and extends almost 0.4 chord in radius. The streamlines coalesce slightly upstream of the vortex, indicating the separation line. It is also interesting to note the formation of another separation line 0.50 chord above the vortex. As a result of the restricted channel formed by the upper strut surface and the neighboring wall, the fluid particles are pushed down, away from the junction, along the strut lower surface. They follow a common path as can be seen by the coalescence of the streamlines there. 


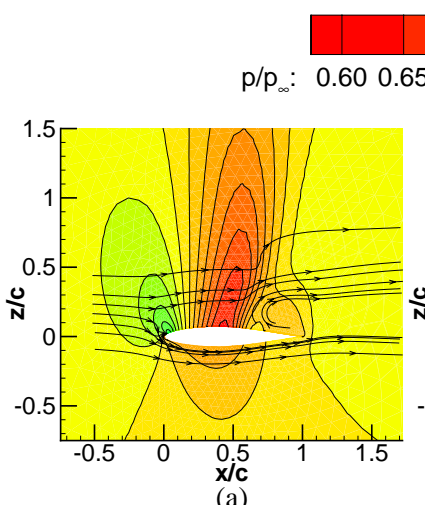

(a)

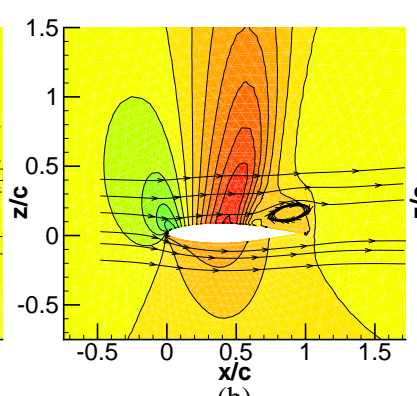

(b)

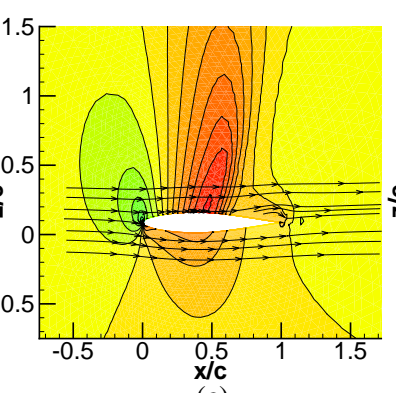

(c)

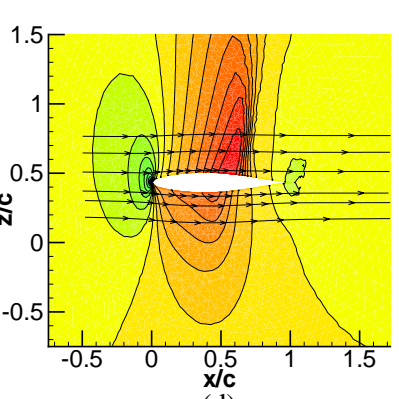

(d)

Figure 5.22: Dissipation of the recirculation vortex for NACA 64A007.5 with $\phi=30^{\circ}$, $M_{\infty}=0.85, \alpha=0^{\circ}, R e_{c}=5.3 \times 10^{6}$ (Navier-Stokes solution) (a) $\eta=0.0002 \%$ (b) $\eta=0.2 \%$ (c) $\eta=1 \%$ (d) $\eta=5 \%$

Results in Figure 5.22 show the dissipation of the recirculation vortex as the distance away from the side wall increases. Although the recirculation region is clearly apparent for the $\eta=0.0002 \%$ and the $\eta=0.2 \%$ stations, it becomes very small at $\eta=1 \%$. A small portion of the fluid particles are deviated in the vicinity of the trailing edge as a result of the presence of the vortex. At $\eta=5 \%$, the recirculation region is completely gone.

We will now do the same exercise as for the NACA 64A005 section by looking at the flowfield around the NACA $64 \mathrm{~A} 007.5$ strut at $\eta=10 \%$. First, for the Euler solutions, the cross section for $\phi=90^{\circ}$ is identical to the one made at $\eta=0.0002 \%$ as expected since the flow is two dimensional for that configuration. The reduction of the strut-wall angle to $\phi=60^{\circ}$ is acompanied by an alleviation of the shock on the upper surface compared to the $\eta=0.0002 \%$ cross section. And on the lower surface, increasing the distance with the side wall contributes to form a shock and create more pressure disturbances in the flowfield. Close to the leading and trailing edges, the contours tend to be symmetric with respect to the chord of the airfoil. The same can be said about the leading edge region for $\phi=30^{\circ}$, but not for the trailing edge, because the shock on the upper surface highly disturbs the flowfield there. As mentioned for the $\phi=60^{\circ}$ case, the distance with the wall plays a central role in the decay of the shock strength on the upper strut surface and in the formation of a shock on the lower surface.

The Navier-Stokes solutions show similar effects as we move away from the side wall. The $\phi=90^{\circ}$ case between viscous side walls shows how quickly the influence of the side wall 


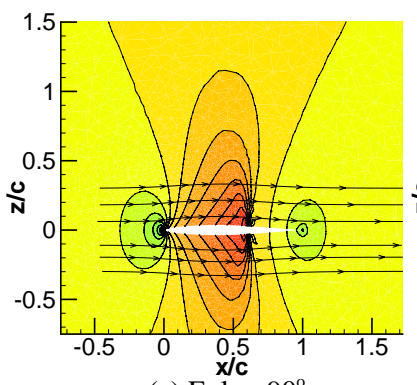

(a) Euler, $90^{\circ}$

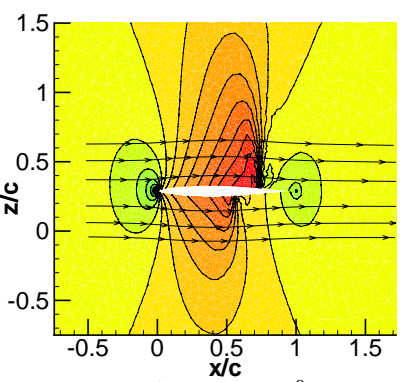

(b) Euler, $60^{\circ}$

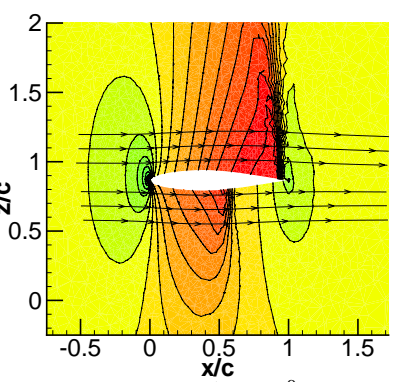

(c) Euler, $30^{\circ}$

$\mathrm{p} / \mathrm{p}_{\infty}: \quad 0.600 .650 .700 .750 .800 .850 .900 .951 .001 .051 .101 .151 .201 .251 .301 .351 .40$

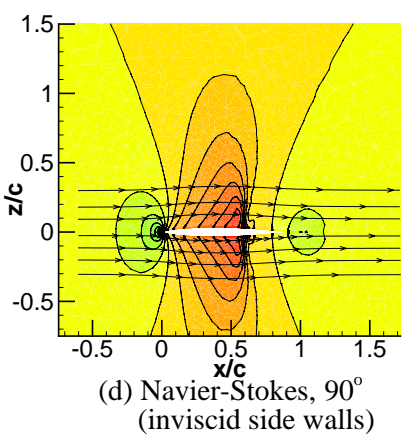

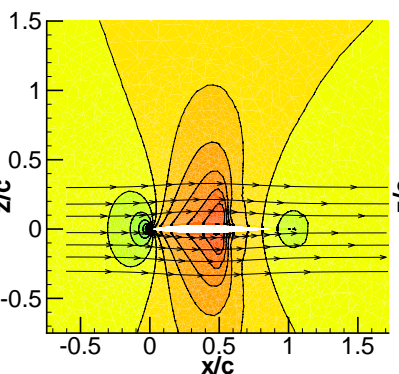

(e) Navier-Stokes, $90^{\circ}$

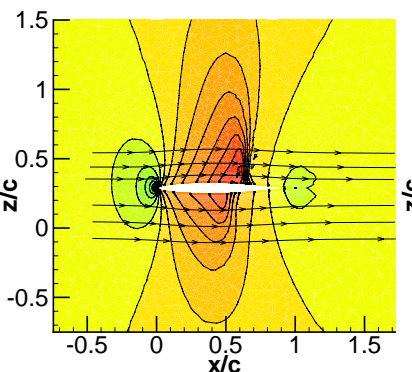

(f) Navier-Stokes, $60^{\circ}$

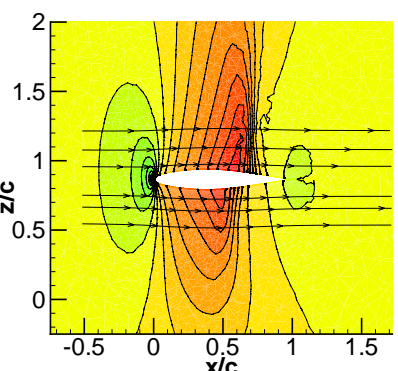

(g) Navier-Stokes, $30^{\circ}$

Figure 5.23: Pressure contours with streamtraces at $\eta=10 \%$ for NACA 64A007.5, $M_{\infty}=0.85, \alpha=0^{\circ}, R e_{c}=5.3 \times 10^{6}-$ Euler solution: (a) $\phi=90^{\circ}$ (b) $\phi=60^{\circ}$ (c) $\phi=30^{\circ}-$ Navier-Stokes solution: (d) $\phi=90^{\circ}$ (inviscid side walls) (e) $\phi=90^{\circ}$ (f) $\phi=60^{\circ}$ (g) $\phi=30^{\circ}$

boundary layer dissipates. The pressure distribution in the flowfield looks already similar to the one for the case between inviscid side walls. The shock on both surfaces is already established. For $\phi=60^{\circ}$, the benefits of the presence of the side wall boundary layer go away as the shock becomes sharper on the upper surface and the disturbnaces become more significant on the lower surface. The $\phi=30^{\circ}$ case shows no sign of the recirculation vortex that was present in the cross section made at $\eta=0.0002 \%$. Instead, the fluid particles are only slightly deviated as they pass near the strut. The shocks on the upper and lower surfaces are apparent.

\subsubsection{Two-Dimensionality of the Flow}

The distance between the walls was chosen to ensure that the flow would be almost twodimensional at the strut mid span. As depicted in the pressure distributions presented in 
Figures 5.4, 5.5, 5.13, and 5.14, the pressure curves on the top and bottom surface of the strut don't lie on top of each other at $\eta=50 \%$. They are very close however, especially for the viscous cases. In order to have a better idea of how close to two-dimensional the results are at mid span, contour plots are made at sections perpendicular to the strut planform instead of making them parallel to the side walls. The pressure contours can be compared in that plane with the results for $\phi=90^{\circ}$ between inviscid walls for the Navier-Stokes calculations and for $\phi=90^{\circ}$ for the Euler solutions.

For convenience, the coordinate system $x-z^{\prime}$ is introduced, where $x$ is in the streamwise direction as always, and $z^{\prime}$ is normal to the wing planform. The origin of the axes is located at the leading edge of the strut where the cross section is made. The results of this exercise for the Euler and Navier-Stokes solutions are shown in Figure 5.24 and 5.25 for the NACA 64A005 and 64A007.5 sections respectively.

For the NACA 64A005 strut section, the Euler solutions are not quite two-dimensional at mid span. This can be noticed for the case with $\phi=30^{\circ}$ where there is a larger region of high speed flow on the strut upper and lower surfaces than for the $\phi=90^{\circ}$ configuration. The Navier-Stokes calculations show the same trend but to a smaller degree. For the cases where $\phi=90^{\circ}$ and $60^{\circ}$, there is a very small difference with the case where the strut is perpendicular to inviscid walls.

If we consider the NACA 64A007.5 section, the Euler solutions for $\phi=60^{\circ}$ and $30^{\circ}$ are not quite two-dimensional at mid span. The contours extend too far away from the strut. The shock appear to be at the same location for all three solutions. Because of the effect of the viscous dissipation, the Navier-Stokes results tend to follow more closely the pattern of the $\phi=90^{\circ}$ case between inviscid side walls. There is no difference between the two $\phi=90^{\circ}$ solutions. The other cases for $\phi=60^{\circ}$ and $30^{\circ}$ show pressure contours that extend further away than for the $\phi=90^{\circ}$ configurations. For the strut-wall angle of $30^{\circ}$, the shock is not as sharp as for the other cases.

Based on these observations, it appears that for the strut-wall angles considered in this study a distance of 5 chords between the parallel walls is satisfactory although a larger distance would provide a flowfield that would be closer to 2-D at mid span. However, this should not have a big impact on the results, particularly for the Navier-Stokes solutions. 


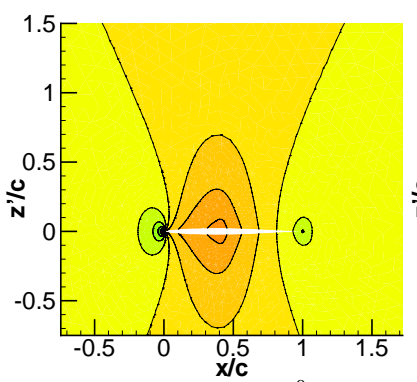

(a) Euler, $90^{\circ}$

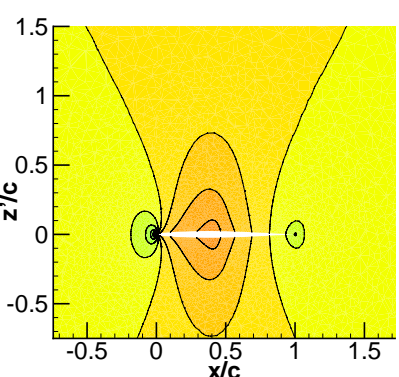

(b) Euler, $60^{\circ}$

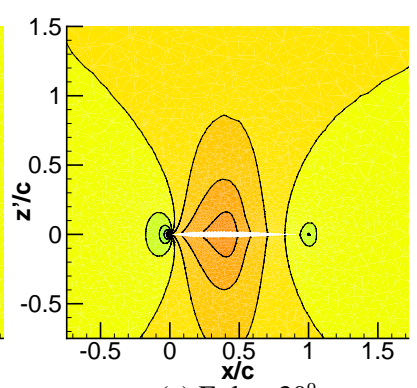

(c) Euler, $30^{\circ}$

$\mathrm{p} / \mathrm{p}_{\infty}: \quad 0.600 .650 .700 .750 .800 .850 .900 .951 .001 .051 .101 .151 .201 .251 .301 .351 .40$

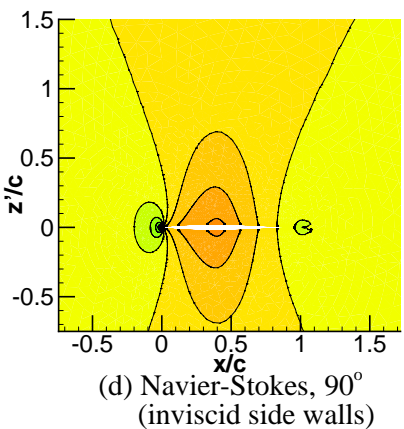

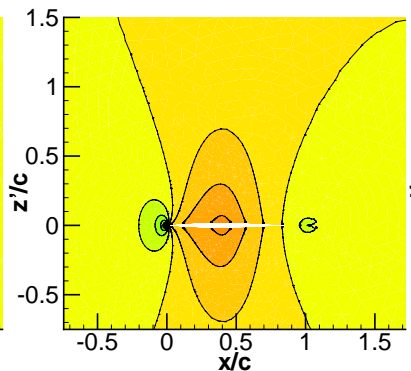

(e) Navier-Stokes, $90^{\circ}$

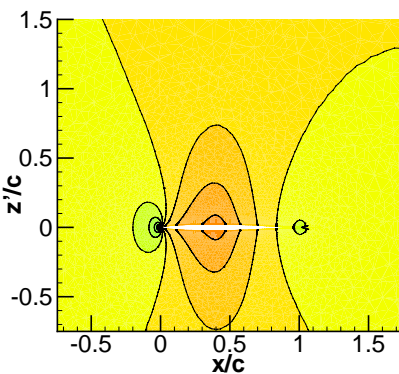

(f) Navier-Stokes, $60^{\circ}$

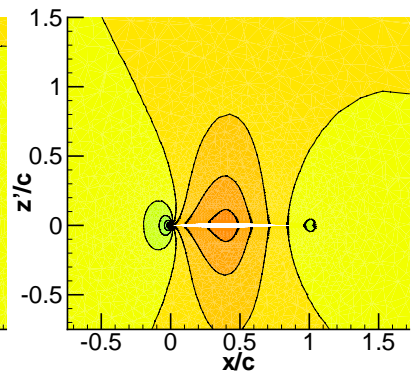

(g) Navier-Stokes, $30^{\circ}$

Figure 5.24: Pressure contours in a plane perpendicular to the strut planform at $\eta=50 \%$ for NACA 64A005, $M_{\infty}=0.85, \alpha=0^{\circ}, R e_{c}=5.3 \times 10^{6}$ - Euler solution: (a) $\phi=90^{\circ}$ (b) $\phi=60^{\circ}$ (c) $\phi=30^{\circ}$ - Navier-Stokes solution: (d) $\phi=90^{\circ}$ (inviscid side walls) (e) $\phi=90^{\circ}$ (f) $\phi=60^{\circ}$ (g) $\phi=30^{\circ}$ 


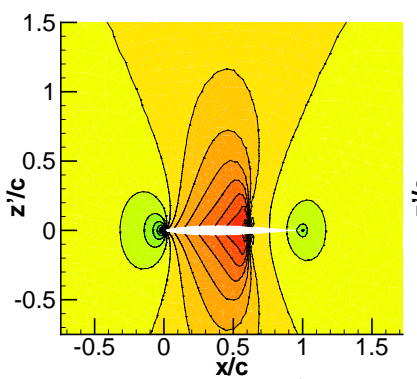

(a) Euler, $90^{\circ}$

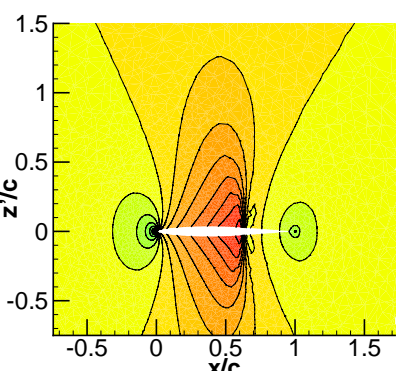

(b) Euler, $60^{\circ}$

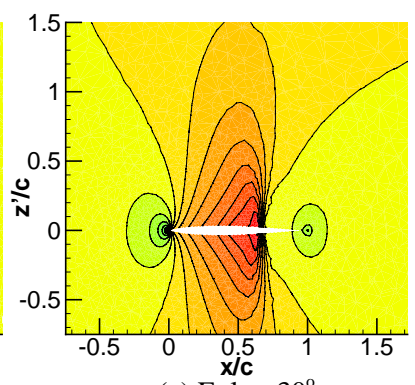

(c) Euler, $30^{\circ}$

$\mathrm{p} / \mathrm{p}_{\infty}: 0.600 .650 .700 .750 .800 .850 .900 .951 .001 .051 .101 .151 .201 .251 .301 .351 .40$

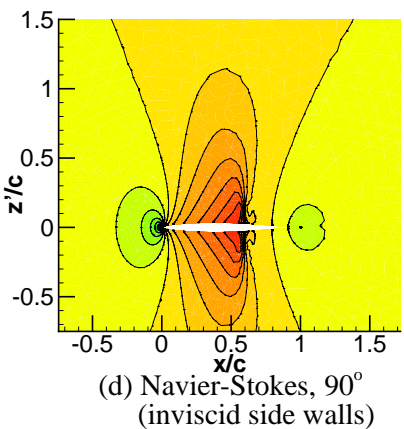

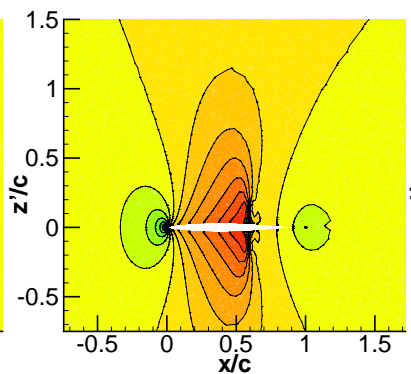

(e) Navier-Stokes, $90^{\circ}$

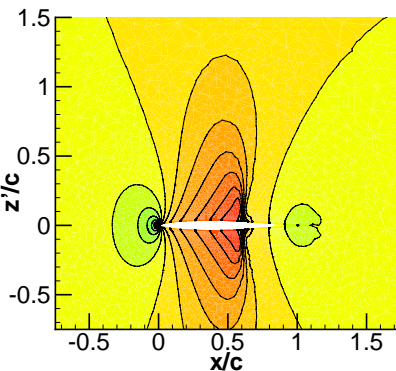

(f) Navier-Stokes, $60^{\circ}$

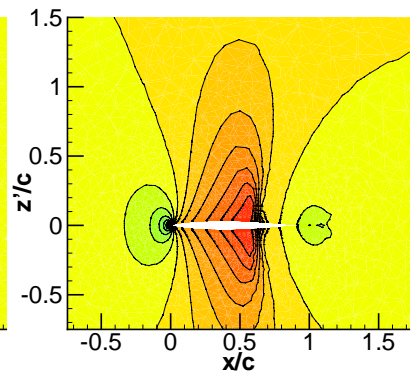

(g) Navier-Stokes, $30^{\circ}$

Figure 5.25: Pressure contours in a plane perpendicular to the strut planform at $\eta=50 \%$ for NACA 64A007.5, $M_{\infty}=0.85, \alpha=0^{\circ}, R_{c}=5.3 \times 10^{6}$ - Euler solution: (a) $\phi=90^{\circ}$ (b) $\phi=60^{\circ}$ (c) $\phi=30^{\circ}$ - Navier-Stokes solution: (d) $\phi=90^{\circ}$ (inviscid side walls) (e) $\phi=90^{\circ}$ (f) $\phi=60^{\circ}$ (g) $\phi=30^{\circ}$ 


\section{Chapter 6}

\section{Challenges in Computational Fluid Dynamics}

The field of CFD has seen major developments in recent years. However, some practical issues remain to be addressed in terms of geometry definition (trailing edge closure), grid generation (presence of slivers and impossibility to close the volume grid), and solution accuracy (grid convergence studies and effect of grid refinement near the leading and trailing edge of airfoil sections). In this chapter, some guidelines and advice developed based on experience in the current study are presented for each of them.

\subsection{Trailing edge closure}

Consider the case of an airfoil with a finite thickness at the trailing edge in transonic flow. The flow expansion around the sharp corner causes a sharp variation in the pressure distribution. This is particularly apparent for Euler calculations. To capture this feature of the flow, the grid has to be refined in that region. In three dimensions, the cost of adding several nodes along the trailing edge can be prohibitive. In addition, the tetrahedral cells along the thickness of the trailing edge tend to be very skewed. For supercritical airfoils, a lot of voids tend to form near the trailing edge region and prevent the volume grid from being completed in some cases. Therefore, it would be nice to find a way to close the trailing edge of the airfoil without affecting too much the value of the aerodynamic coefficients computed. As depicted in Figure 6.1, this can be achieved in several ways: 
1. Insert the trailing edge point at the intersection of the extrapolated upper and lower airfoil surfaces (Figure 6.1b). The chord of the airfoil is not preserved and this has to be taken into account in calculating the aerodynamic coefficients such as $C_{l}, C_{d}$, and $C_{m}$. For some supercritical airfoils, the extrapolated upper and lower surfaces coincide at a point located too far from the airfoil. This is due to the fact that the surfaces are almost parallel near the trailing edge. Thus, an extension of those surfaces could potentially go to infinity.

2. Combine the last point on the upper and lower surfaces and replace them by a point located midway between them (Figure 6.1c).

3. Join the next to last point on the lower surface to the last point on the upper surface. Drop the last point on the lower surface also (Figure 6.1d).

4. Join the next to last point on the upper surface to the last point on the lower surface. Drop the last point on the upper surface also (Figure 6.1e).

5. Move the upper and lower airfoil surfaces towards each other in such a way that they coincide at a point at the trailing edge (Figure 6.1f). In order to preserve the camber line, the displacement of the surfaces can be done linearly with $x$ as:

$$
z_{i}^{\prime}=\left\{\begin{array}{l}
z_{i}-\frac{1}{2}\left(\frac{t}{c}\right)\left(x_{i}-x_{L E}\right) \text { for } i=1,2, \ldots n_{\text {upper }} \text { on upper surface } \\
z_{i}+\frac{1}{2}\left(\frac{t}{c}\right)\left(x_{i}-x_{L E}\right) \text { for } i=1,2, \ldots n_{\text {lower }} \text { on lower surface }
\end{array}\right.
$$

where $z_{i}^{\prime}$ and $z_{i}$ represent the new and old airfoil ordinates respectively, $x_{i}$ the abscissa of each point on the airfoil surface, $t$ the thickness of the airfoil at the trailing edge, and $x_{L E}$ the abscissa of the leading edge of the airfoil ( $x_{L E}=0$ usually). The upper and lower surfaces are represented by $n_{\text {upper }}$ and $n_{\text {lower }}$ points respectively.

Rivers and Wahls [118] discussed some of the alternatives presented above for closing the trailing edge.

In the analysis of the interference drag of a strut intersecting a flat wall presented in Chapter 5, the flow past airfoils of the NACA 64A- family was analyzed. To assess the effect of the trailing edge closure methods on the pressure distribution and the aerodynamic coefficients, we solved the transonic flow past the NACA 64A007.5 airfoil. The airfoil is symmetric and has a $t / c$ of $7.5 \%$. The next to last point is located at $x / c=0.99$. The 


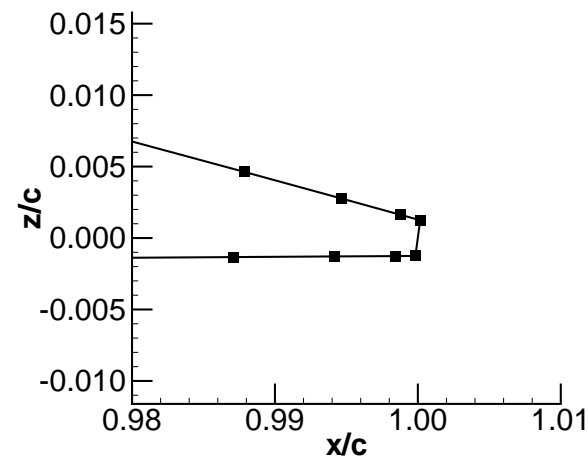

(a)

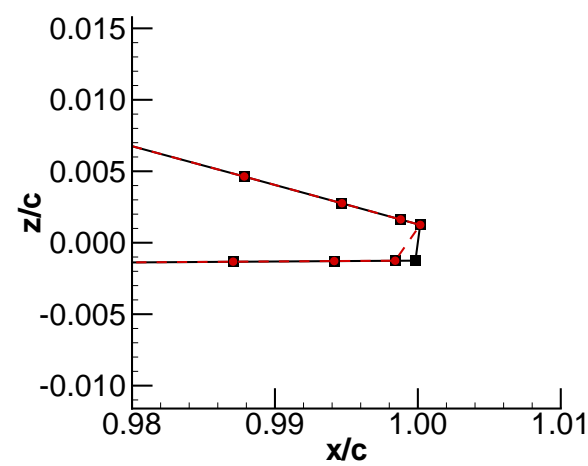

(d)

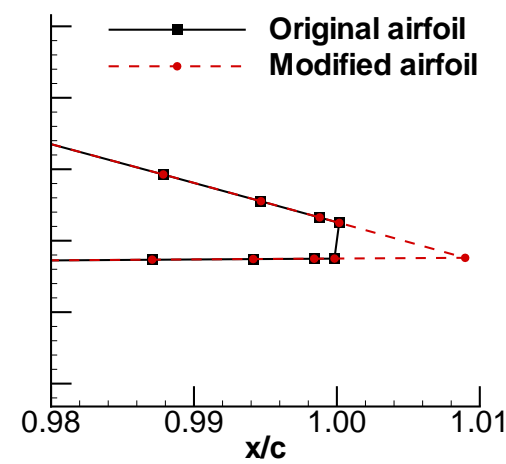

(b)

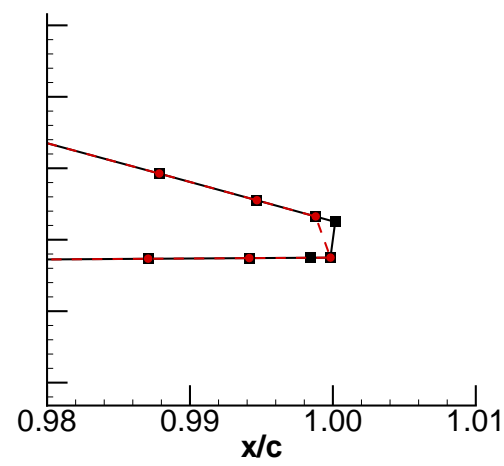

(e)

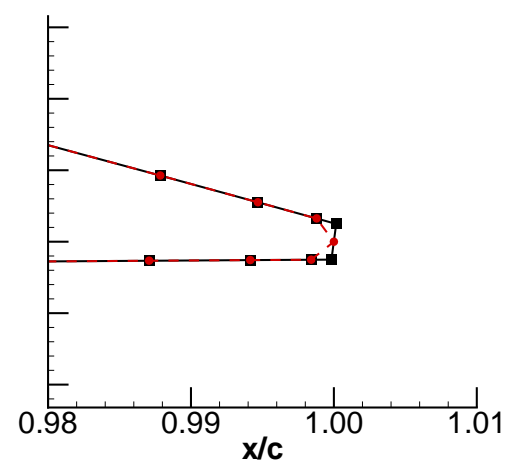

(c)

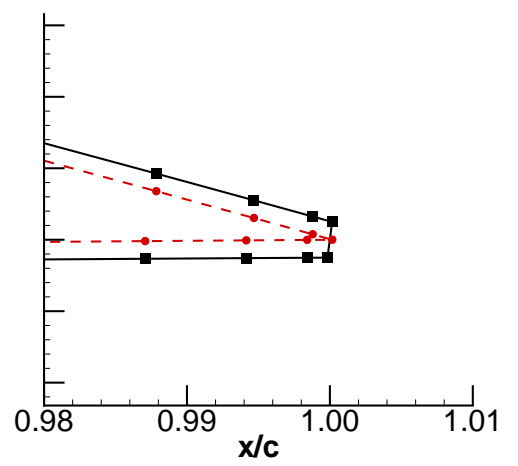

(f)

Figure 6.1: Methods to close the trailing edge (a) Original airfoil (b) Method 1: Extrapolation of the upper and lower surfaces (c) Method 2: Midpoint (d) Method 3: Removal of the last point on the lower surface (e) Method 4: Removal of the last point on the upper surface (f) Method 5: Displacement of the upper and lower surfaces using a linear variation 
freestream conditions are set to $M_{\infty}=0.85$ with an angle of attack $\alpha=0^{\circ}$ and a Reynolds number $R e_{c}=5.3 \times 10^{6}$. Since the airfoil is symmetric, Methods 3 and 4 are equivalent. The generated unstructured grids use the same number of grid points on the surface of the airfoil for all cases. However, for the case of the original airfoil with a finite thickness at the trailing edge, grid points are added along the segment forming the flat trailing edge.

The pressure distributions are shown in Figure 6.2 for the solution of the inviscid Euler equations. The original airfoil shape exhibits a strong shock at $x / c=0.55$. As the flow goes around the corner at the trailing edge, the pressure decreases sharply. When applying Method 1, the shock is predicted at the same location. However, the pressure at the trailing edge increases as the flow smoothly leaves the airfoil surface. The result obtained with Method 2 is consistent with Method 1 except for the bump in the pressure distribution around $x / c=0.99$. This is caused by the abrupt change of the surface slope in that region, thus forcing the flow to expand. The worst flow characteristics happen with Methods 3 and 4 where the shock location varies over a range of $20 \%$ of the chord length. This asymmetry in the pressure produces unwanted lift. Method 5 predicts the shock upstream of the shock location for the original airfoil. The flow leaves smoothly the airfoil surface at the trailing edge as in the case of the Method 2.

The Navier-Stokes solutions for the same airfoil geometries are pictured in Figure 6.3. As opposed to the Euler results, the calculations generally show a better agreement with the pressure distribution of the original airfoil shape. The viscous forces act at the trailing edge to blend away the sharp pressure variations. This mechanism is responsible for the improvement in the results for Methods 3 and 4. However, the asymmetry between the upper and lower surface is still responsible for a non-negligible lift force for those cases.

The lift and drag coefficients are presented in Table 6.1 for the Euler and Navier-Stokes computations. The results for Method 1 are corrected to take into account the fact that the extrapolation of the upper and lower surfaces causes the airfoil chord to increase by $1 \%$. The lift coefficient $C_{l}$ should be zero for all cases because the airfoil is symmetric and the freestream is at an angle of attack $\alpha=0^{\circ}$. Of all the methods shown, Method 5 predicts a lift coefficient that is closest to zero. The remaining difference can be explained by the grid itself. There are some inaccuracies when the forces are determined by integration. If we don't consider the results of Method 2, the Euler calculations yield a drag coefficient within 0.0002 of each other. For the Navier-Stokes solutions, the skin friction drag component is constant for all cases. The variation in the pressure drag among the methods can be 


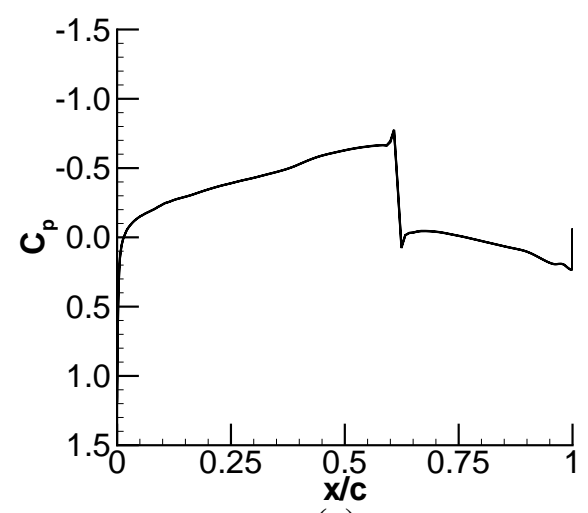

(a)

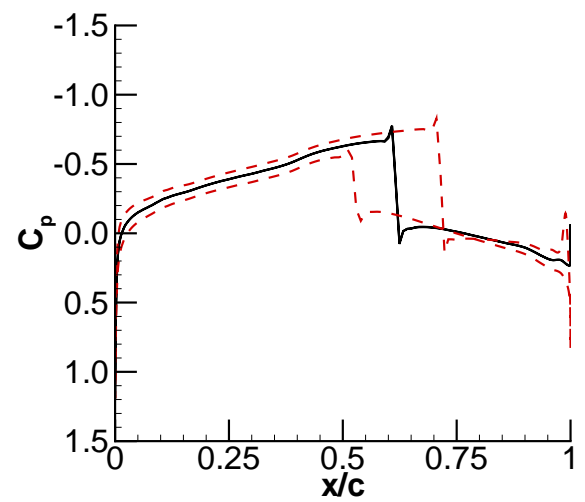

(d)

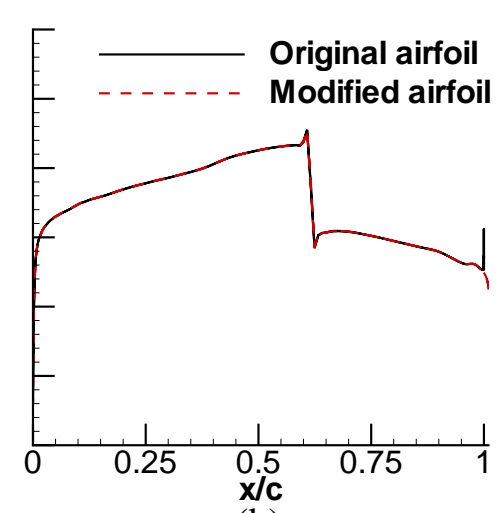

(b)

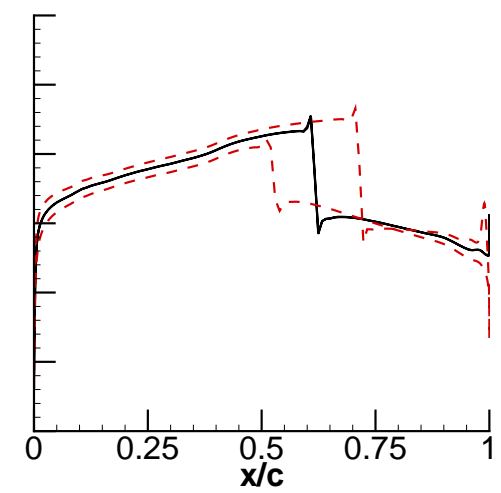

(e)

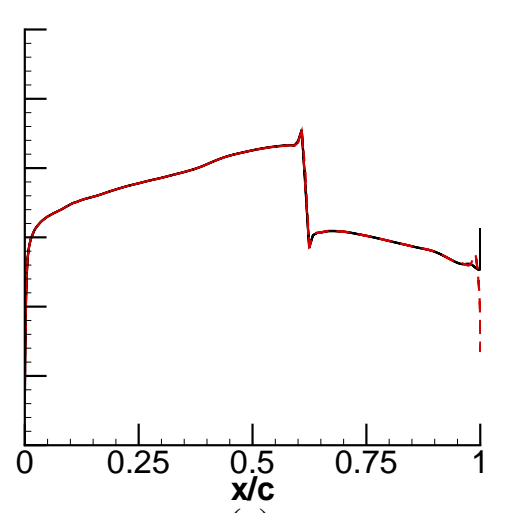

(c)

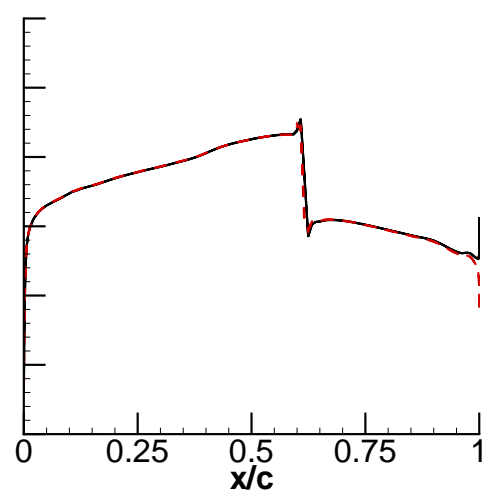

(f)

Figure 6.2: Pressure distribution with trailing edge closure for the NACA 64A007.5 airfoil, $M_{\infty}=0.85, \alpha=0^{\circ}$ (Euler solution) (a) Original airfoil (b) Method 1: Extrapolation of the upper and lower surfaces (c) Method 2: Midpoint (d) Method 3: Removal of the last point on the lower surface (e) Method 4: Removal of the last point on the upper surface (f) Method 5: Displacement of the upper and lower surfaces using a linear variation 


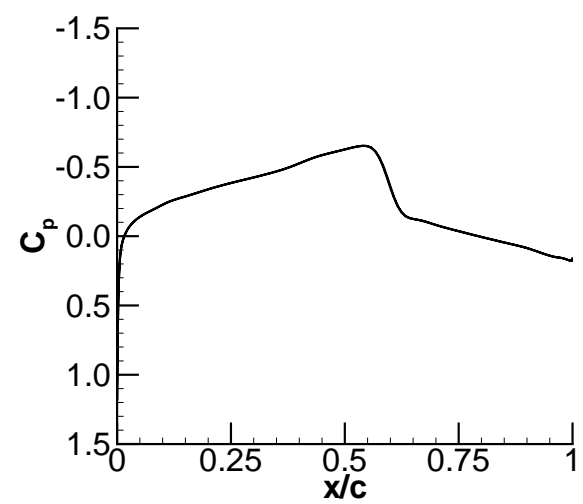

(a)

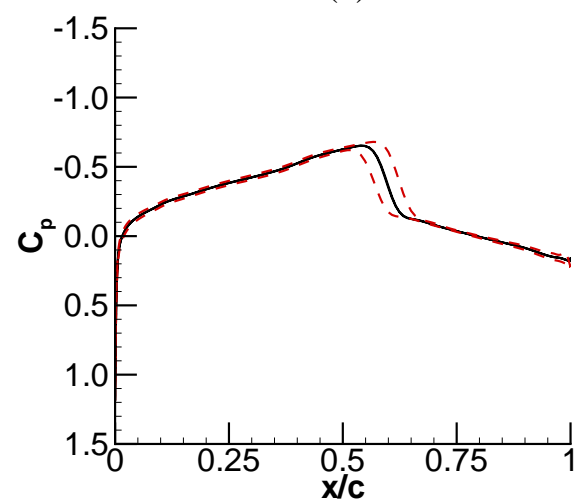

(d)

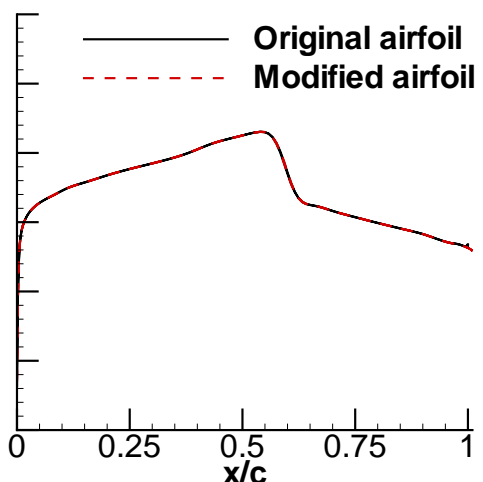

(b)

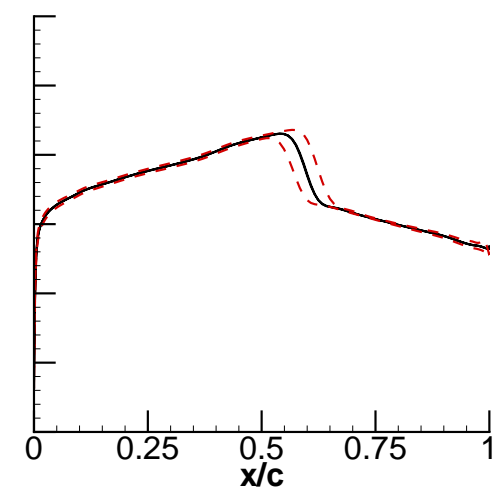

(e)

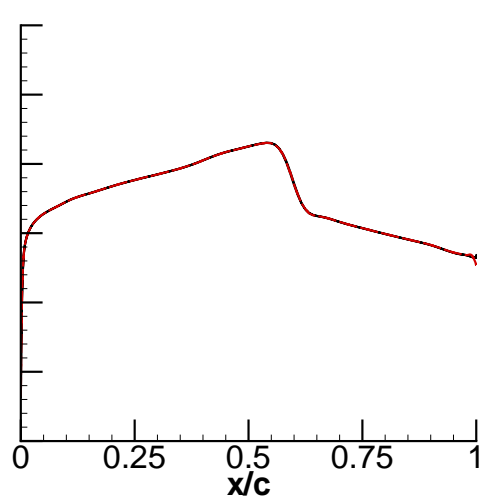

(c)

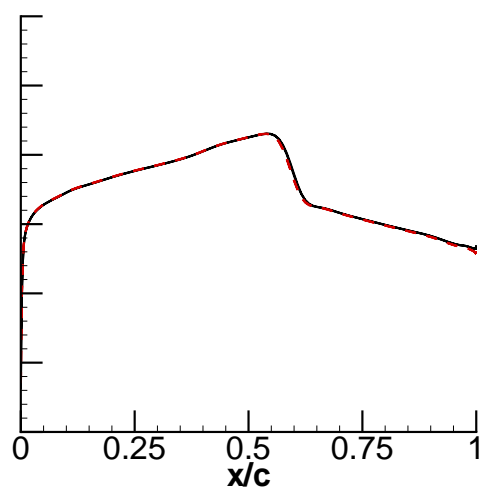

(f)

Figure 6.3: Pressure distribution with trailing edge closure for the NACA 64A007.5 airfoil, $M_{\infty}=0.85, \alpha=0^{\circ}, R e_{c}=5.3 \times 10^{6}$ (Navier-Stokes solution) (a) Original airfoil (b) Method 1: Extrapolation of the upper and lower surfaces (c) Method 2: Midpoint (d) Method 3: Removal of the last point on the lower surface (e) Method 4: Removal of the last point on the upper surface (f) Method 5: Displacement of the upper and lower surfaces using a linear variation 
Table 6.1: Lift and drag coefficients for various trailing edge closure methods

\begin{tabular}{|c||c|c||c|c|c|c|}
\hline \multicolumn{1}{|c||}{ Case } & \multicolumn{2}{c||}{ Euler } & \multicolumn{4}{c|}{ Navier-Stokes } \\
\cline { 2 - 7 } & $C_{l}$ & $C_{d}$ & $C_{l}$ & $C_{d_{p}}$ & $C_{d_{v}}$ & $C_{d_{t o t}}$ \\
\hline \hline Original & $-4.65 \times 10^{-4}$ & 0.0043 & $-7.88 \times 10^{-4}$ & 0.0058 & 0.0058 & 0.0116 \\
Method 1 & $-5.35 \times 10^{-4}$ & 0.0041 & $-8.27 \times 10^{-4}$ & 0.0056 & 0.0058 & 0.0114 \\
Method 2 & $-1.36 \times 10^{-3}$ & 0.0042 & $-5.02 \times 10^{-4}$ & 0.0057 & 0.0058 & 0.0115 \\
Methods 3-4 & -0.195 & 0.0065 & $-5.74 \times 10^{-2}$ & 0.0060 & 0.0058 & 0.0118 \\
Method 5 & $4.34 \times 10^{-5}$ & 0.0041 & $-2.61 \times 10^{-4}$ & 0.0056 & 0.0058 & 0.0114 \\
\hline
\end{tabular}

explained by the differences in the shock location. Overall, the Navier-Stokes results agree within 0.0004 for the total drag coefficient. Finally, Methods 3 and 4 yield the worst results because of their relatively high lift coefficients. As opposed to the Euler calculations, the Navier-Stokes computations for those methods yield a drag coefficient that is closer to the one of the original airfoil because the prediction of the shock location was improved.

No definite conclusion can be made about these approaches because only one set of freestream conditions was tested and no extensive analyses were made about other airfoil shapes. However, it appears that Methods 3 and 4 should be avoided. For the computations performed on the strut-braced wing airplane in Chaper 4, the airfoil sections were closed with Method 2 because the airfoil sections employed had upper and lower surfaces that were very close to each other near the trailing edge. For the interference drag analyses of strut-wall configurations presented in Chapter 5, Method 5 was employed to close the trailing edge of the strut sections.

\subsection{Grid convergence studies}

Unstructured grid methodology provides a lot of flexibility in analyzing complex configurations. However, it has some drawbacks in terms of the accuracy of the flow solution, especially if no mesh adaptivity is used. It is difficult to perform grid convergence studies for a given problem. A grid convergence study consists in computing the flow solutions on systematically finer grids. Typically, the lift and drag coefficients are plotted in terms of the inverse of the total number of mesh points. As the grid becomes finer, it is expected that the aerodynamic coefficients will reach an asymptotic value. This strategy is employed to determine what level of grid refinement is necessary to achieve a certain level of accuracy. 
This method is easily applicable to structured grids as coarser grids can be obtained from a very fine grid by ignoring every other point in each direction. For unstructured grids, this approach is not feasible. Independent sets of grids have to be generated and it is often hard to derive coarser grids that are consistent with an existing fine grid.

In two dimensions, AFLR2 allows the generation of coarser grids relatively easily because the number of points on the airfoil surface and on the far-field boundary can be specified directly. However, in three dimensions with VGRIDns, the approach is not as straightforward, because both the spacing $s$ and the strength $a_{n}$ of a grid source can be adjusted. For inviscid meshes such as the ones employed in Chapter 4, the spacing is doubled for every source in the mesh for each level of mesh coarsening. For viscous grids, VGRIDns is sometimes not able to find a suitable unstructured grid to meet the prescribed grid point distribution. The strength of the sources need to be adjusted to ensure a smooth growth of the mesh in the field. In this research, the approach that was most often preferred for viscous grids was to increase the strength of the corner sources until a desired number of mesh points on the surface of the body was obtained. Since the spacing of the corner sources in the far-field is usually large, making those sources stronger propagates their effect farther in the far-field and thus coarsens the grid even on the surface of the body. The only disadvantage of this method is that the spacing in the far-field is not changed. Over the long run, this method proved to be more robust than any other in terms of grid generation for viscous grids.

To make life easier, one could think of using an extension to AFLR2 in three dimensions. Such a package exists to generate inviscid and viscous grids and contains the graphical user interface SolidMesh [119] and the grid generation code AFLR3. Both codes were developed at Mississippi State University. SolidMesh allows the definition of points, curves, and surfaces on 2-D and 3-D geometries. The grid spacing is specified in the field and the unstructured grid is generated by AFLR3 using the advancing-front/local-reconnection methodology [98, 99]. Right now, one of the disadvantages of this product is that it doesn't have the capability to allow grid stretching along directions of small gradients as opposed to VGRIDns. This causes the size of the grids obtained with SolidMesh and AFLR3 to be very large in comparison with similar grids generated with VGRIDns. 


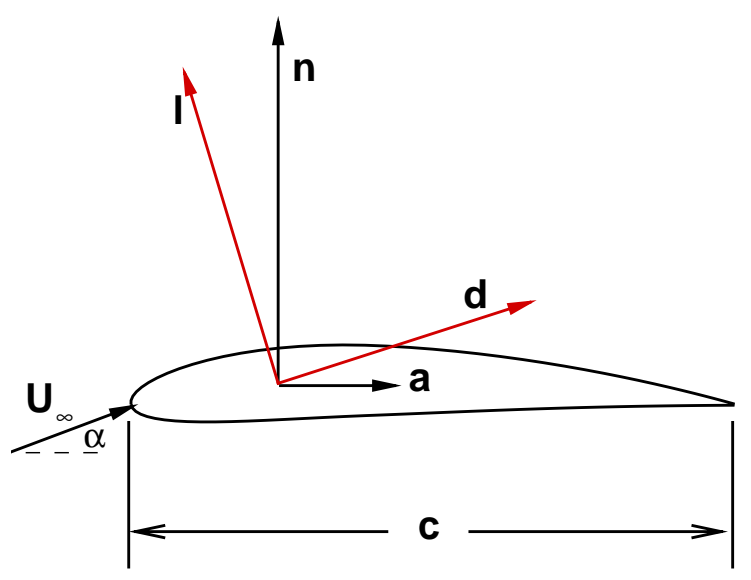

Figure 6.4: Forces acting on a 2-D airfoil

\subsection{Grid generation in the vicinity of the leading edge and the trailing edge}

The development of CFD tools has allowed an improvement in the computation of the flowfield and the associated aerodynamic coefficients. In general, the lift coefficient is a quantity that is predicted accurately by current methods. However, it can sometimes be very difficult to obtain good agreement of the drag coefficient with experimental data. On the one hand, this can be due to the experiment itself: blockage effect, side wall boundary layers, accuracy of the measurement system, etc. On the other hand, poor predictions can be a result of the grid quality, the turbulence model employed, etc. In this section, we will look at some of the issues that can have a noticeable impact on the pressure distributions and thus the determination of the drag.

Let us review how the lift and drag coefficients are calculated. This will help to understand the subsequent discussion better. Consider an airfoil with a chord $c$ at an angle of attack $\alpha$ as shown in Figure 6.4. The forces acting on the airfoil in the axial and normal directions are denoted by $a$ and $n$ respectively. To transform those components into the usual lift $l$ and drag $d$ forces, the following rotation is performed [120]: 


$$
\begin{aligned}
& l=n \cos \alpha-a \sin \alpha \\
& d=n \sin \alpha+a \cos \alpha
\end{aligned}
$$

These quantities are non-dimensionalized with respect to a reference area $S_{\text {ref }}=c \times 1$ and a reference dynamic pressure $q_{\infty}=\frac{1}{2} \rho_{\infty} U_{\infty}^{2}$ to get $C_{l}$ and $C_{d}$. In terms of the pressure coefficient on the upper surface $C_{p, u}$ and on the lower surface $C_{p, l}$, the contribution of the pressure to the lift and drag coefficients is determined by integrating the difference in pressure coefficients along the airfoil surface from the leading edge $L E$ to the trailing edge $T E$. Using Eq. 6.1 and 6.2, we get from Ref. [120]:

$$
\begin{aligned}
& C_{l}=\frac{1}{c}\left[\left(\int_{L E}^{T E}\left(C_{p, l}-C_{p, u}\right) d x\right) \cos \alpha-\left(\int_{L E}^{T E}\left(C_{p, u}-C_{p, l}\right) d z\right) \sin \alpha\right] \\
& C_{d}=\frac{1}{c}\left[\left(\int_{L E}^{T E}\left(C_{p, l}-C_{p, u}\right) d x\right) \sin \alpha+\left(\int_{L E}^{T E}\left(C_{p, u}-C_{p, l}\right) d z\right) \cos \alpha\right]
\end{aligned}
$$

If the airfoil is at an angle of attack $\alpha=0^{\circ}$, the above equations reduce to:

$$
\begin{aligned}
& C_{l}=C_{n}=\frac{1}{c} \int_{L E}^{T E}\left(C_{p, l}-C_{p, u}\right) d x \\
& C_{d}=C_{a}=\frac{1}{c} \int_{L E}^{T E}\left(C_{p, u}-C_{p, l}\right) d z
\end{aligned}
$$

Consider the case of the symmetric NACA 64A007.5 airfoil discussed in Section 6.1. The freestream conditions are taken as $M_{\infty}=0.85$ and $\alpha=0^{\circ}$. Two inviscid grids are generated with AFLR2 with 512 points along the airfoil surface. For the fine grid, the grid spacing at the leading edge is specified as $1.2 \times 10^{-3}$ chord and the mesh contains a total of 9968 nodes. The spacing at the leading edge for the coarse grid is $3 \times 10^{-3}$ and the grid contains 9808 nodes. The grids are pictured in Figure 6.5.

The Euler equations are solved using the flow solver FUN2D and the pressure distribution for both cases is depicted in Figure 6.6. The lift and drag coefficients are displayed in Table 6.2. The lift coefficient is very close to zero as expected. However, the drag coefficient on the coarse grid overpredicts the value on the fine grid by as much as $7 \%$. Looking at the pressure distribution in Figure 6.6a, there is no apparent difference between the two curves. How can the difference in drag be explained? The answer lies in the definition of the pressure drag coefficient from Eq. 6.6:

$$
C_{d}=\frac{1}{c} \int_{L E}^{T E}\left(C_{p, u}-C_{p, l}\right) d z
$$



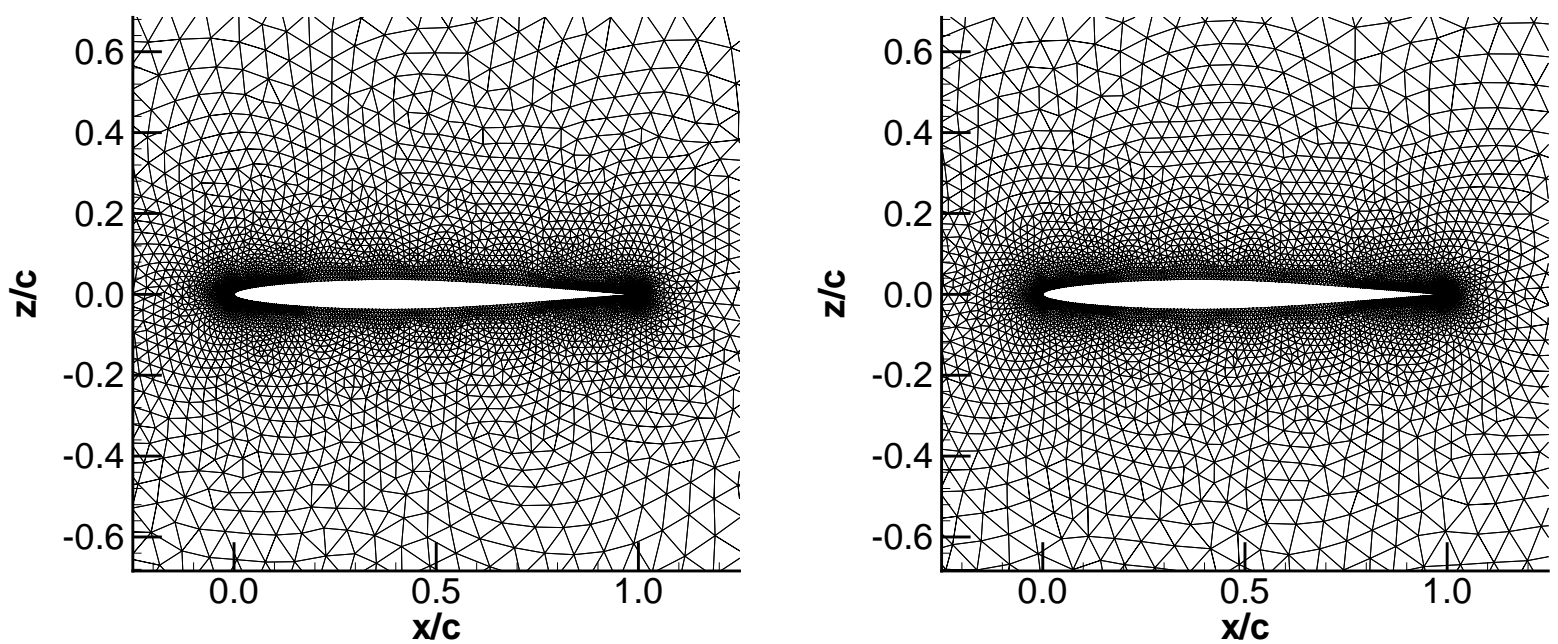

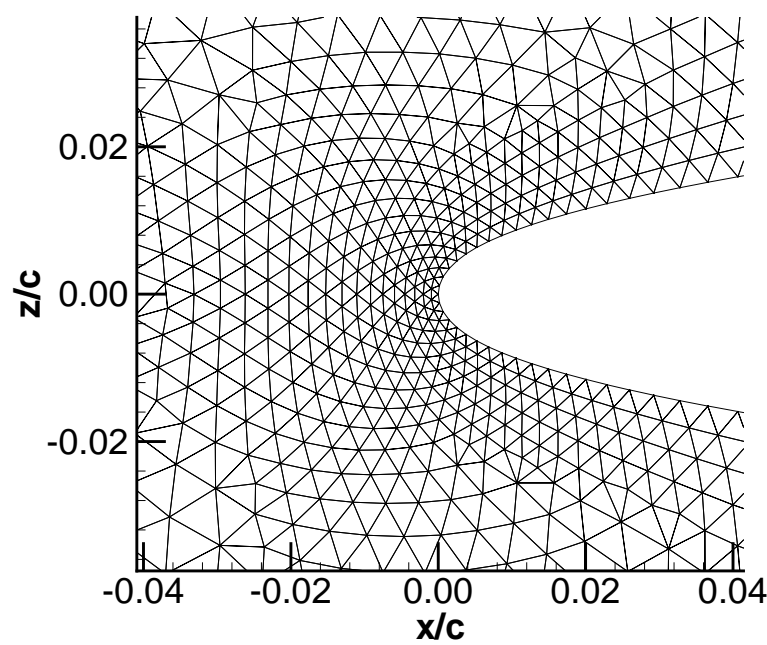

(a)

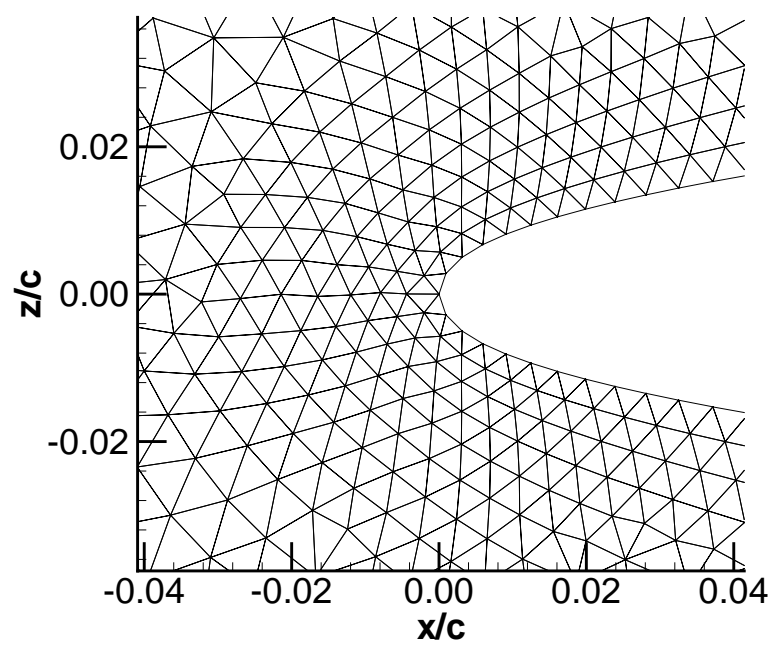

(b)

Figure 6.5: Refinement of the inviscid grid at the leading edge of the NACA 64A007.5 airfoil (a) Fine grid with close-up view (b) Coarse grid with close-up view

Table 6.2: Lift and drag coefficients for various grid refinements at the leading edge of the NACA 64A007.5 airfoil, $M_{\infty}=0.85, \alpha=0^{\circ}$

\begin{tabular}{|c||c|c|}
\hline Case & $C_{l}$ & $C_{d}$ \\
\hline \hline Fine grid & $-7.48 \times 10^{-4}$ & 0.0041 \\
Coarse grid & $-6.41 \times 10^{-4}$ & 0.0044 \\
\hline
\end{tabular}




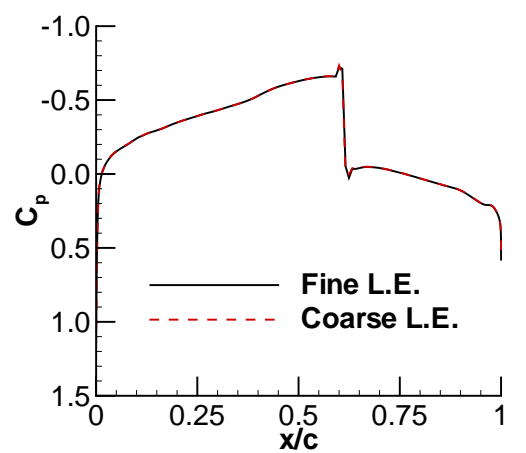

(a)

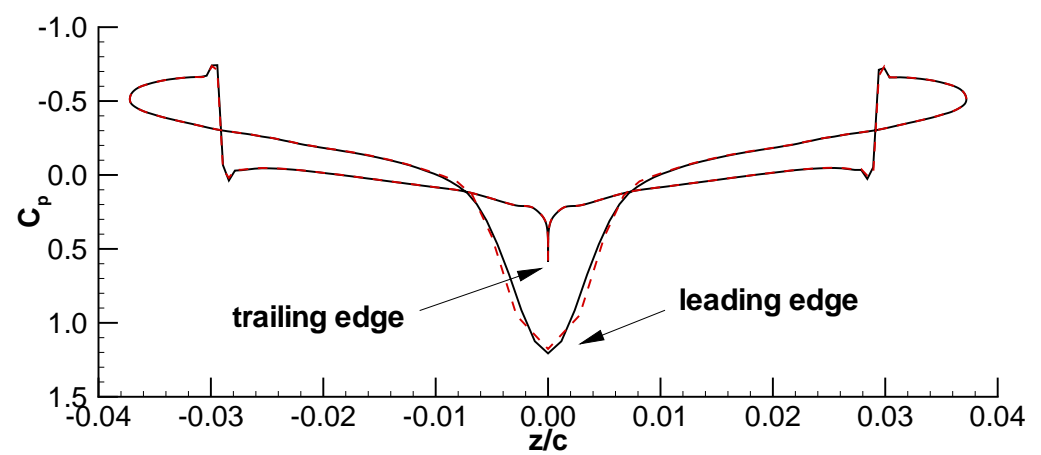

(b)

Figure 6.6: Effect of the grid refinement at the leading edge of the NACA 64A007.5 airfoil, $M_{\infty}=0.85, \alpha=0^{\circ}$ (Euler solution) (a) Pressure coefficient as a function of the coordinate $x / c(\mathrm{~b})$ Pressure coefficient as a function of the coordinate $z / c$

The pressure drag is obtained by integrating along the height of the airfoil. At the leading edge, where the pressure varies rapidly, the airfoil z-coordinate also changes rapidly. It is necessary to resolve that region very well because it has a significant impact on the final value of $C_{d}$. Figure $6.6 \mathrm{~b}$ shows the pressure distributions in an unconventional way, the horizontal axis being the z-coordinate of the airfoil surface. This plot is called a "thrust loop". The computation of the area enclosed by a curve yields the drag coefficient for the corresponding mesh. Looking closely at the pressure distributions in the vicinity of the airfoil leading edge, it is obvious that there is a slight difference in the $C_{p}$ curves for the coarse and fine grids. This can explain the difference in drag noted earlier. The shock appears to be resolved accurately with the grids employed here.

For the sake of discussion, consider the points on the airfoil surface where $z / c=0.01$. Those points correspond to $x / c=0.015$ and $x / c=0.865$. To be able to capture accurately the suction peak and its effect on the drag coefficient, this means that there has to be about as many grid points in the region where $0 \leq x / c \leq 0.015$ as in the region where $0.865 \leq x / c \leq 1$. The conclusion to this exercise is that the leading edge region is of primary importance in the calculation of the drag coefficient. Poor discretization of the geometry in that vicinity can have a detrimental impact on the results.

There is another interesting phenomenon related to how fine the grid is near the leading edge. Consider an airfoil of the NACA $64 \mathrm{~A}$ family with $t / c=5 \%$, thus corresponding to the name NACA 64A005. Grids can be generated for that airfoil section using VGRIDns. VGRIDns is a three-dimensional grid generator but it can be employed to obtain two-dimensional grids 


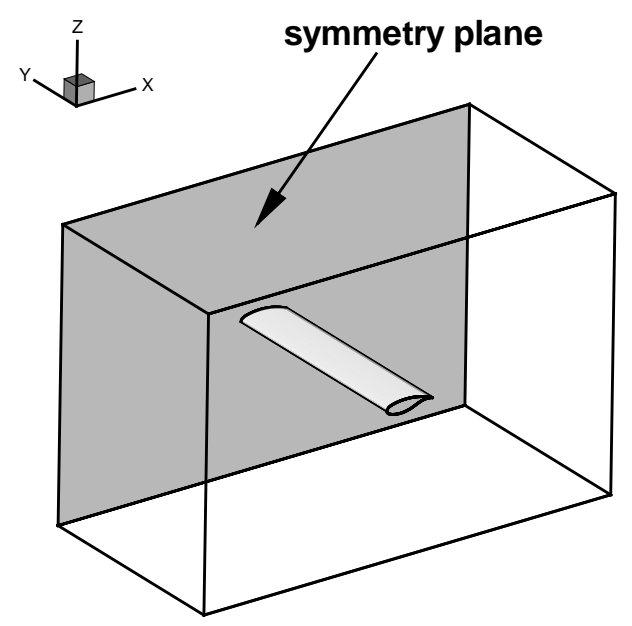

Figure 6.7: Generation of a 2-D grid with VGRIDns

in a simple fashion. As shown in Figure 6.7, a wing with a given span is enclosed between the walls of the computational box. The mesh on the symmetry plane can be extracted and transformed to a two-dimensional grid suitable for the flow solver FUN2D. That way, some features of the grid can be tested quickly rather than having to analyze a three-dimensional case.

For the NACA 64A005 airfoil, two viscous grids are generated with VGRIDns. Figure 6.8a shows the finest grid which has 189 points along the airfoil surface and a total of 9215 nodes in the field. The mesh depicted in Figure 6.8b is coarser, with 171 points along the airfoil surface and a total of 7856 nodes. The spacing prescribed for each grid source is the same for both grids. The viscous grids have identical parameters for the growth of the viscous layers. The only difference in the parameters used to obtain these grids is the strength $a_{n}$ of the source located at the leading edge of the airfoil. For the finest grid, $a_{n}$ is twice as large as it is for the coarser mesh.

The freestream conditions for this analysis are $M_{\infty}=0.85, \alpha=0^{\circ}$ with a Reynolds number of $R e_{c}=5.3 \times 10^{6}$. The Reynolds-averaged Navier-Stokes equations are solved using FUN2D. The lift and drag coefficients computed for each solution are shown in Table 6.3. The lift coefficient is negligible as expected. The total drag coefficient is almost identical for both cases, the main difference arising from the integration of the pressure to calculate the pressure drag. This becomes clearer when looking at the pressure distribution presented in 


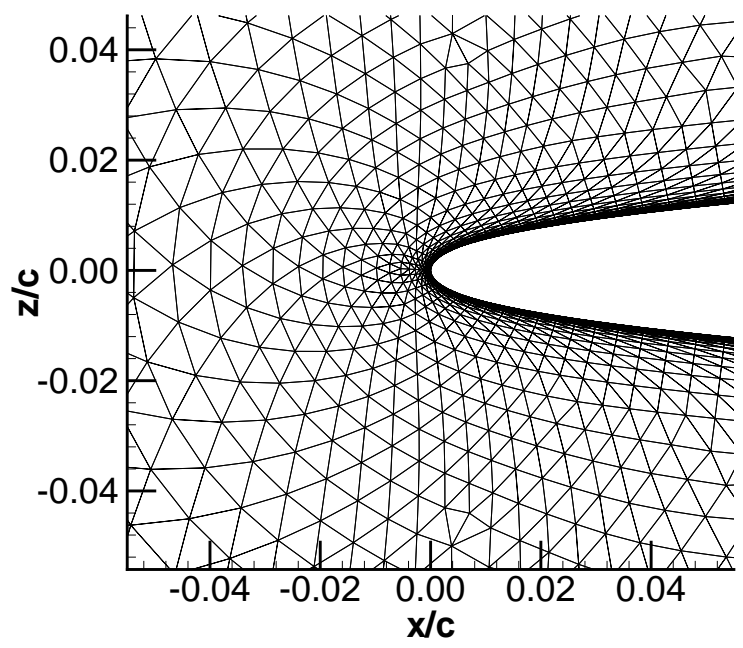

(a)

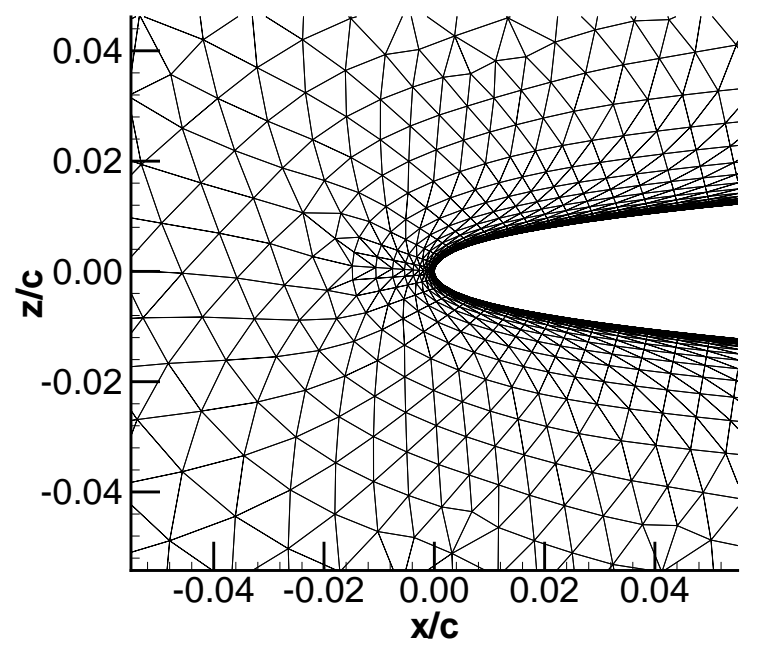

(b)

Figure 6.8: Grid refinement at the leading edge of the NACA 64 A005 airfoil (a) Fine grid (b) Coarse grid

Table 6.3: Lift and drag coefficients for various grid refinements at the leading edge of the NACA 64A005 airfoil, $M_{\infty}=0.85, \alpha=0^{\circ}, R e_{c}=5.3 \times 10^{6}$

\begin{tabular}{|c||c|c|c|c|}
\hline Case & $C_{l}$ & $C_{d_{p}}$ & $C_{d_{v}}$ & $C_{d_{\text {tot }}}$ \\
\hline \hline Fine grid & $-3.57 \times 10^{-4}$ & 0.0013 & 0.0057 & 0.0071 \\
Coarse grid & $-3.95 \times 10^{-5}$ & 0.0015 & 0.0057 & 0.0072 \\
\hline
\end{tabular}

Figure 6.9. The close-up view of the pressure near the leading edge of the airfoil shows a very strange variation in the $C_{p}$ coefficient. This bump is present in the solution on the fine grid, but with a much smaller amplitude. Although not shown here, the thicker NACA 64A007.5 section has the same problem, but to a smaller degree. The grids generated with AFLR2 don't exhibit that behavior. The current state of the art flow solver requires that careful attention be paid to generating suitable grids, otherwise the solution process might diverge or the solution might be inaccurate.

For viscous grids, it is very important that the viscous layers be generated perpendicularly to the airfoil surface to ensure an accurate computation of the flow solution. Looking closely at the grids in the vicinity of the leading edge in Figure 6.8, it appears that the grid lines that are supposed to be normal to the airfoil surface deviate significantly from the normal direction as we move closer to $x / c=0$. This phenomenon is more obvious in the case 


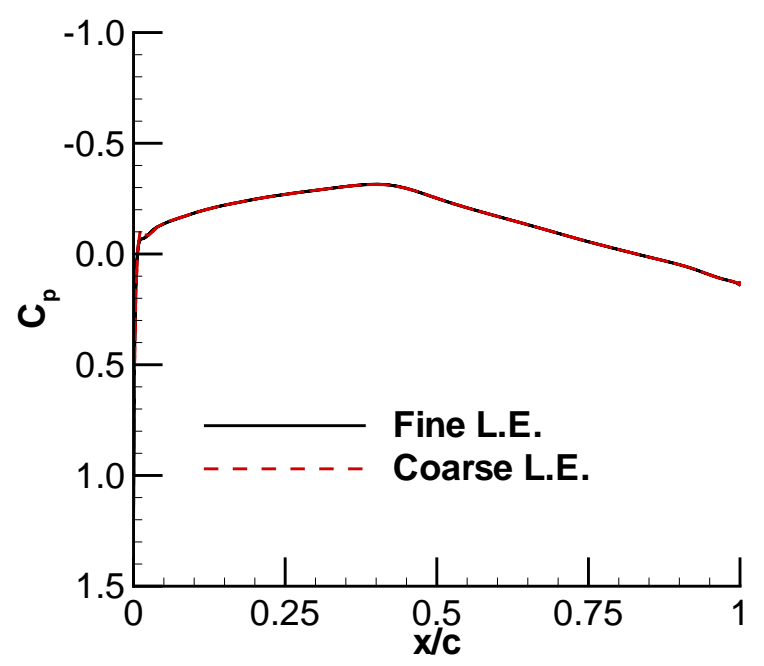

(a)

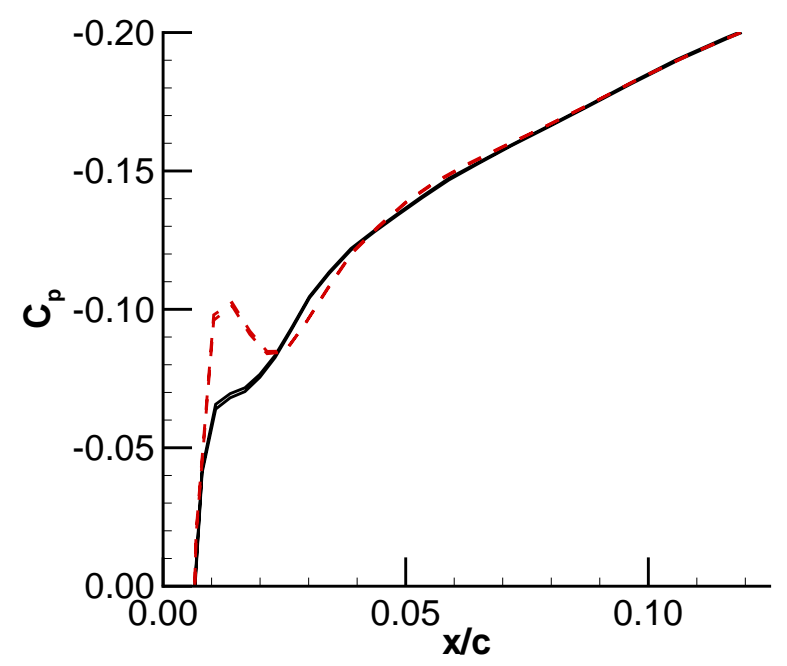

(b)

Figure 6.9: Effect of the grid refinement at the leading edge of the NACA 64A005 airfoil, $M_{\infty}=0.85, \alpha=0^{\circ}, R e_{c}=5.3 \times 10^{6}$ (Navier-Stokes solution) (a) Pressure distribution on the airfoil surface (b) Close-up view in the vicinity of the leading edge

of the coarse grid. VGRIDns computes the surface normals, smoothes them, before finally generating the viscous layers. More points along the surface means more normals. More normals improves the grid quality in regions where there is a sharp corner such as at the trailing edge of an airfoil. It is also true here because the airfoil is thin and has a rather sharp leading edge. More normals gives more flexibility to the grid generation code to handle abrupt variations in the body surface. The normal grid lines are thus more perpendicular to the body surface. As opposed to VGRIDns, the grid generation code AFLR2 adds more normals to the existing ones when a sharp corner is detected.

An extension of the discussion presented above will now focus on the grid refinement at the trailing edge of the airfoil. This time, the airfoil section considered is the NACA 64A007.5 section employed before. As shown in Figure 6.10, two viscous grids are obtained with VGRIDns in the same fashion as for the previous case. The only difference between them is the spacing $s$ at the trailing edge: it is set to 0.001 for the finer grid and to 0.004 for the coarser one. For the finer mesh, the airfoil surface is discretized with 135 points and the total number of nodes in the field is 6,292. The coarser grid has 129 nodes on the surface and 6,101 total nodes.

The flow solver FUN2D solves the Reynolds-averaged Navier-Stokes equations for the 


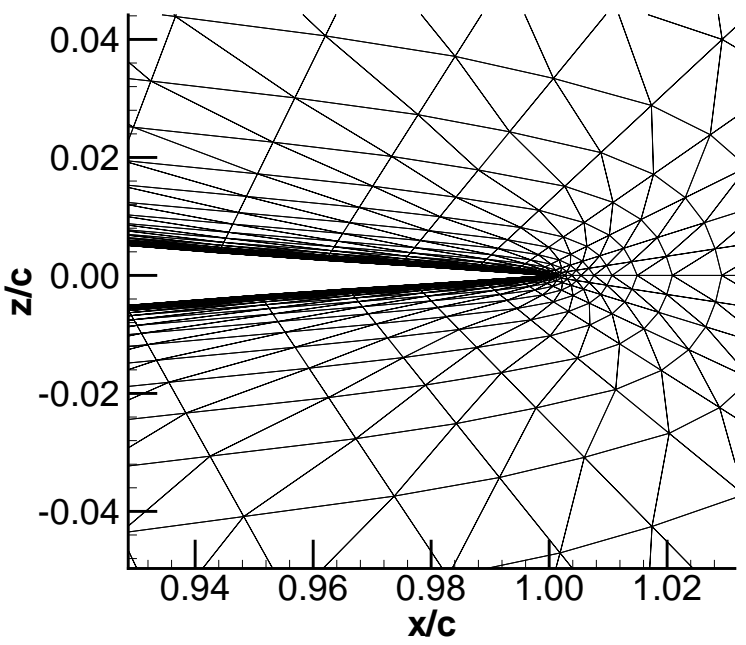

(a)

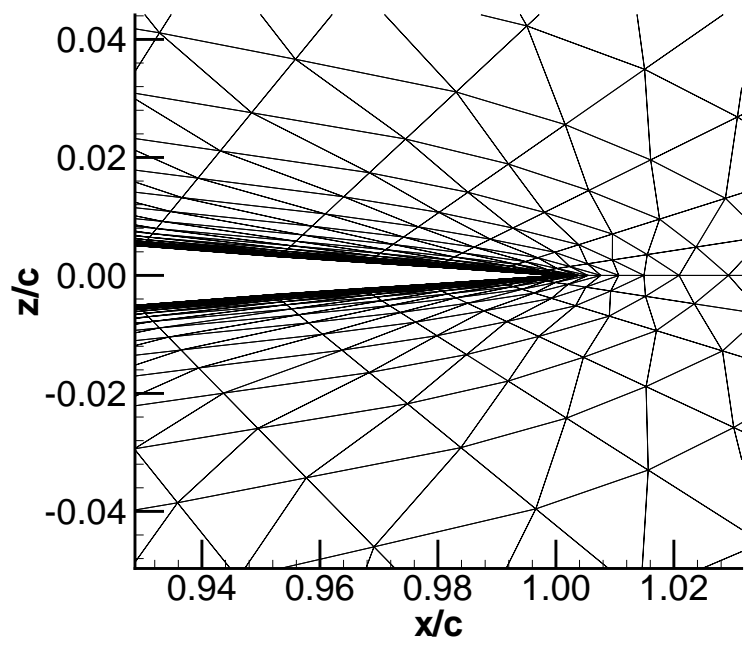

(b)

Figure 6.10: Grid refinement at the trailing edge (a) Fine grid (b) Coarse grid

Table 6.4: Lift and drag coefficients for various grid refinements at the trailing edge of the NACA 64A007.5 airfoil, $M_{\infty}=0.85, \alpha=0^{\circ}, R e_{c}=5.3 \times 10^{6}$

\begin{tabular}{|c||c|c|c|c|}
\hline Case & $C_{l}$ & $C_{d_{p}}$ & $C_{d_{v}}$ & $C_{d_{\text {tot }}}$ \\
\hline \hline Fine grid & $4.17 \times 10^{-4}$ & 0.0059 & 0.0055 & 0.0114 \\
Coarse grid & $3.19 \times 10^{-4}$ & 0.0059 & 0.0055 & 0.0114 \\
\hline
\end{tabular}

freestream conditions set as $M_{\infty}=0.85, \alpha=0^{\circ}$, and $R e_{c}=5.3 \times 10^{6}$. In Table 6.4, the lift coefficient is very small and the drag coefficient for both analyses is identical. However, there is a difference in the pressure distributions pictured in Figure 6.11. In the region near the trailing edge, the pressure suddenly decreases. This is more dramatic in the case of the coarser mesh. Again, looking at the viscous layers in Figure 6.10, we can see that the normal grid lines are not perpendicular to the surface as we get closer to the trailing edge. For the finer mesh, they are better than for the coarser mesh but not perfect. This could explain the variation in the pressure in the vicinity of the airfoil trailing edge.

\subsection{Impossibility to Close the Volume Grid}

As mentioned in Section 3.1.2, it is sometimes impossible to complete the unstructured volume grid because the corner sources have a grid spacing that is too coarse or they are too 


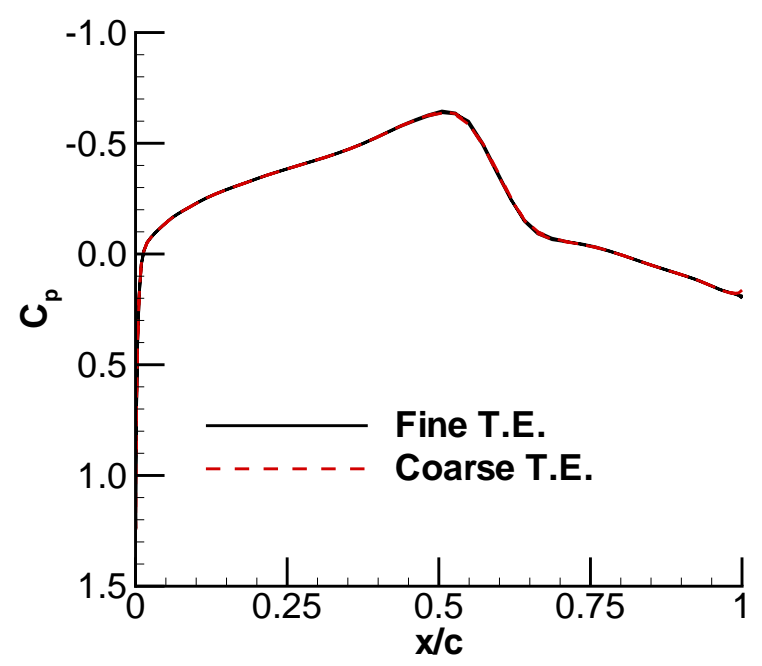

(a)

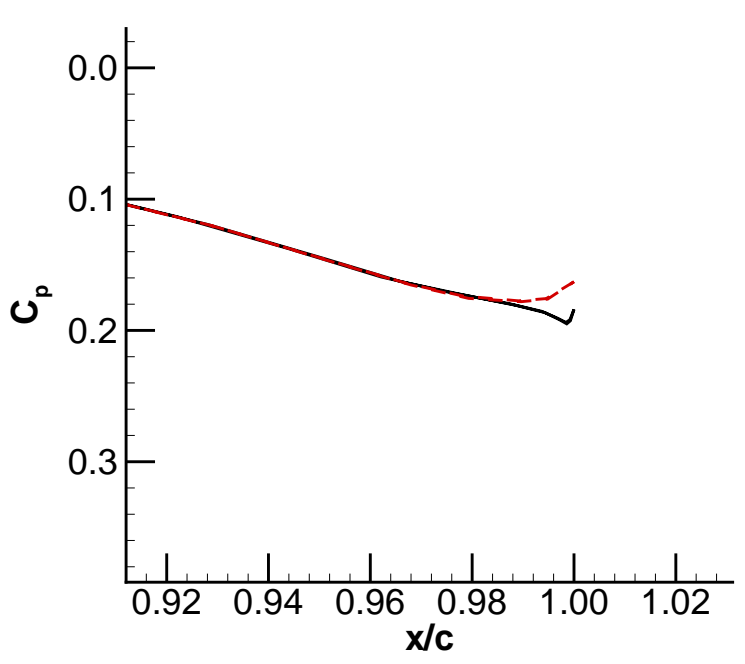

(b)

Figure 6.11: Effect of the grid refinement at the trailing edge of the NACA 64A007.5 airfoil, $M_{\infty}=0.85, \alpha=0^{\circ}, R e_{c}=5.3 \times 10^{6}$ (Navier-Stokes solution) (a) Pressure distribution on the airfoil surface (b) Close-up view in the vicinity of the trailing edge

strong. In that case, the only way out is to go back to the GridTool files and specify new grid parameters for the sources.

There are other instances where the problem is not due to those parameters. There can be grid crossing in the mesh. In order to generate the unstructured volume grid, VGRIDns takes the patches defined by the user and transforms them to a planar surface. The surface triangulation is performed on the transformed patch. Once that step is done, the patches are mapped back to their original shape. However, there is no guarantee that the triangulated patch follows the original geometry perfectly. It is often necessary to project the triangulated patches back onto the original geometry to ensure that all the triangles lie on the surface.

In some instances, such as for the case of a wing with a cusped and thin trailing edge, that projection step is mandatory. Otherwise, there is a risk of seeing the upper and lower triangulated surfaces cross. This is depicted in Figure 6.12. If there is such an overlap, the volume grid generation will fail because VGRIDns will try to grow some cells inside the geometry. The projection ensures the integrity of the mesh.

However, projecting the triangulated patches is sometimes not enough. Often, more points need to be added to the curves bounding each patch. By adding more points, the curves can't deviate as much from the original geometry when they get splined by VGRIDns. 


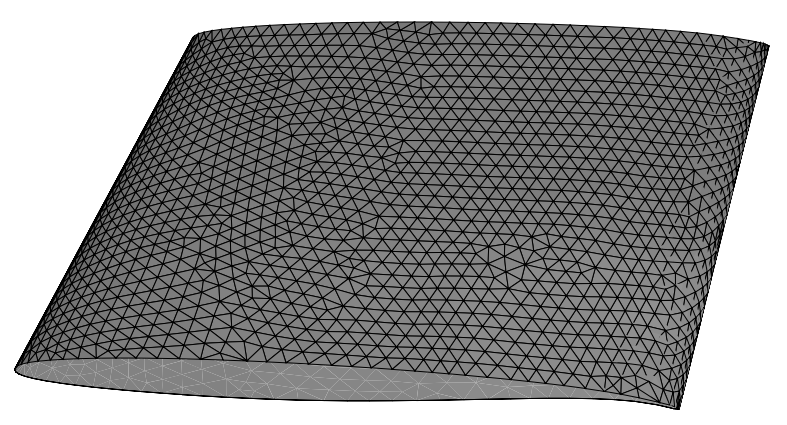

(a)

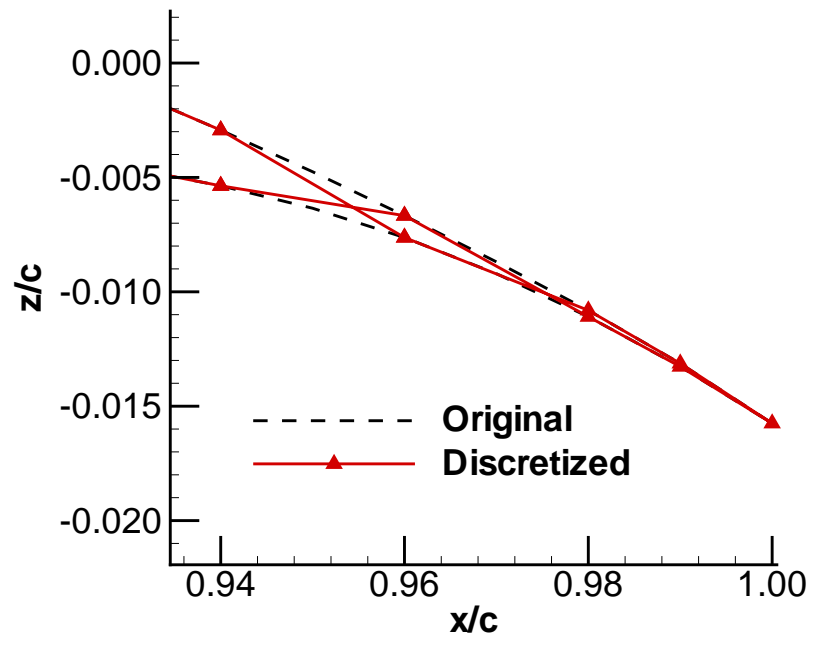

(b)

Figure 6.12: Grid crossing problem (a) Triangulation of a wing planform (b) Comparison of the original and the discretized geometries at the trailing edge

The trailing edge, especially in the vicinity of the wing tip, is a region where this approach likely needs to be applied.

Another possible source of problem is the machine accuracy. When generating viscous grids, the distance between the viscous layers is controlled by the following equation [101]:

$$
\delta_{i}=\delta_{1}\left[1+r_{1}\left(1+r_{2}\right)^{i-1}\right]^{i-1}
$$

where $\delta_{1}$ is the grid spacing at the wall. Consider the configurations discussed in Chapter 5 . If the distance $\delta_{1}$ is a too small value such as $1 \times 10^{-6}$ chord instead of the value of $1 \times 10^{-5}$ chord utilized, VGRIDns can't complete the mesh because it seems to run into limitations with the accuracy of the computer. This is the main reason why $\delta_{1}=1 \times 10^{-5}$ chord was employed instead of $1 \times 10^{-6}$ chord for the three-dimensional meshes.

Finally, another possibility related to the impossibility of closing the volume grid is the memory allocated for the grid generation by VGRIDns. The bulk of memory is allocated during run time based on the maximum number of nodes the user specifies. However, some internal arrays used for the grid generation seem to have dimensions independent of the number of nodes specified by the user. For large grids, this can cause the program to break down. For some configurations such as for the strut at 30 or 60 degrees between parallel walls, the volume grid generation was successful in less than $10 \%$ of the runs. 


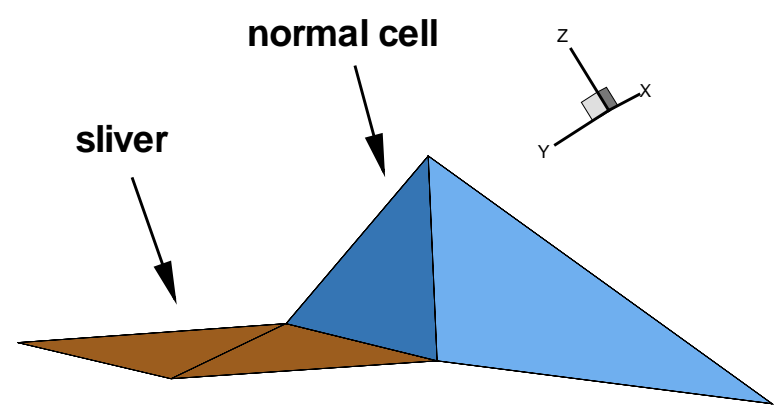

(a)

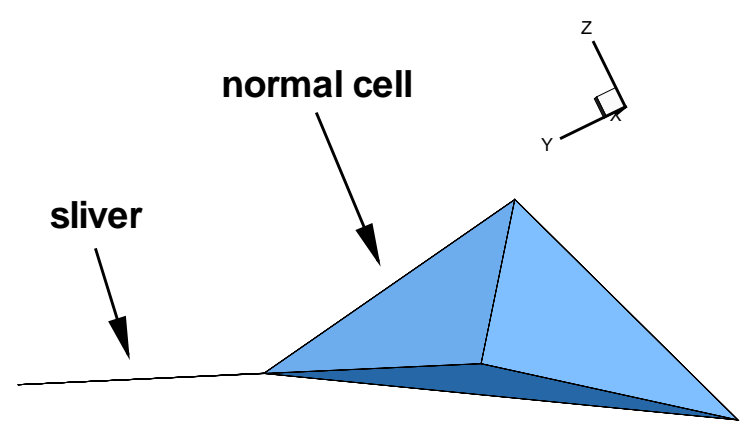

(b)

Figure 6.13: A sliver next to a normal cell (a) Perspective view (b) View where the sliver has negligible thickness

\subsection{Slivers}

As discussed in the previous sections, the level of grid refinement of the mesh in regions of strong gradients has a significant impact on the accuracy of the results computed. But another factor, the poor grid quality, can introduce inaccuracies in the solution and create convergence problems for the flow solver employed.

The presence of "slivers" among the thousands of tetrahedral cells can in some instances prevent the solution from reaching convergence. Slivers are degenerated flat cells that occur in the field when grids are generated for viscous flow solutions in particular. A sliver is compared to a normal cell in Figure 6.13. The thickness of the slivers is so small that the gradients necessary to evaluate the viscous terms are computed erroneously in those cells. This in turn corrupts the CFD solution and causes the flow solver to stop unexpectedly. The employment of a limiter in the flow solver doesn't prevent the problem from occurring.

As described at length in Section 3.2.5, the implicit Euler scheme implemented in the flow solver can be written as:

$$
\left(\frac{V}{\Delta t}+\frac{\partial \mathbf{R}^{n}}{\partial \mathbf{Q}}\right) \Delta \mathbf{Q}^{n}=-\mathbf{R}^{n}
$$

where $V$ is the volume of the cell, $\mathbf{R}$ is the residual, $\mathbf{Q}$ is the vector of conserved variables, and 
$\frac{\partial \mathbf{R}}{\partial \mathbf{Q}}{ }^{n}$ is the Jacobian. The problem with slivers is that their large angles produce inaccuracies in the evaluation of the gradients for the viscous terms. The gradients of the inviscid terms are computed with a least-squares procedure, but for the viscous terms, Green's theorem is employed. Consider the utilization of Green's theorem to compute the gradient of $\phi$ :

$$
\nabla \phi=\frac{1}{V} \sum_{S} \phi \hat{\mathbf{n}} \Delta S
$$

where $V$ is the volume of the tetrahedral element, $\hat{\mathbf{n}}$ is the normal to the face, and $\Delta S$ is the face area. For a sliver, the main problem is that the volume $V$ of the element tends to a very small quantity. In that case, $\nabla \phi$ will become a very large number or be undetermined.

There are several ways to evaluate the quality of an unstructured grid. Marcum [99] used a sliver quality measure $Q_{1}$ given by:

$$
Q_{1}=6 \sqrt{2} \frac{V}{L^{3}}
$$

where $L$ is the average edge length of the cell and $V$ is the cell volume. $Q_{1}$ is zero for a completely flat cell. The skewness quality measure $Q_{2}$ is defined as:

$$
Q_{2}=\frac{9 \sqrt{3}}{8} \frac{V}{R^{3}}
$$

where $R$ is the radius of the circumsphere of the cell. The circumsphere is the sphere of smallest radius in which the cell can be enclosed. In that case, all the vertices of the cell lie on the surface of the circumsphere. $Q_{2}$ is sensitive to skewed elements whereas $Q_{1}$ is able to detect sliver cells effectively.

Another way of detecting slivers consists in calculating the internal angles inside tetrahedral elements [108]. Consider the tetrahedron depicted in Figure 6.14 with the normals associated with each face of the cell. For a sliver such as the one shown in Figure 6.13, at least one pair of normals will have their direction almost coincide with each other. The dot product of those vectors will be very close to one. We can use the dot product to calculate the interior angle $\gamma$ made by two faces of the cell in the following way:

$$
\gamma=180^{\circ}-\cos ^{-1}\left(\hat{\mathbf{n}}_{i} \cdot \hat{\mathbf{n}}_{j}\right)
$$

where $\gamma$ is in degrees.

Consider the case of a strut with a thickness-to-chord ratio $t / c=7.5 \%$ at an angle of $60^{\circ}$ with respect to two parallel walls. The results for that configuration were presented 


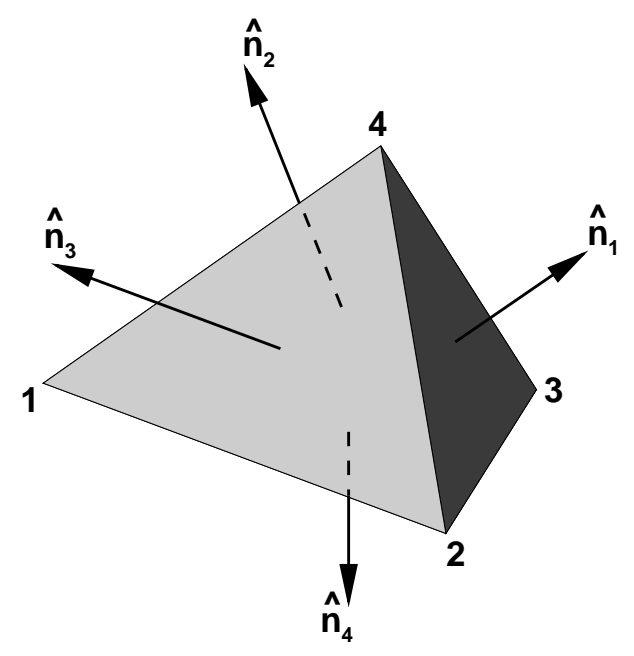

Figure 6.14: Tetrahedral cell and the normal associated with each face

in Chapter 5. An inviscid grid with 1,980,185 cells (351,674 nodes) and a viscous grid containing 5, 965, 997 cells (1, 015, 709 nodes) were generated for that configuration. For the viscous grid, the side walls and the strut were considered as viscous surfaces where the no-slip condition was applied.

For each grid, the maximum interior angle within each cell of the mesh was computed. All the cells with one interior angle greater or equal to 178 degrees were flagged. The location of the flagged cells is shown in Figure 6.15. The inviscid grid contains only 143 slivers. They are located near the leading edge of the strut. Those cells never caused any problem to the CFD code because the grid was not employed to compute viscous flows. The viscous grid has a total of 5,678 slivers located primarily near the trailing edge of the strut. Although few slivers are near the wall-strut junction, monitoring the convergence of the flow solution [108] reveals that they are responsible for the breakdown of the flow solver.

We can look at the distribution of the tetrahedral cells according to the sliver quality measure $Q_{1}$, the skewness quality measure $Q_{2}$, and the maximum angle within each cell. The results are shown in Figure 6.16 for the inviscid grid described above and in Figure 6.17 for the viscous grid. To explain the meaning of these charts, we will consider the inviscid grid first. The bar charts for $Q_{1}$ and $Q_{2}$ indicate the fraction of cells with a given value of the grid quality measure in 0.05 increments. For example, the bar chart for $Q_{1}$ highlights the 

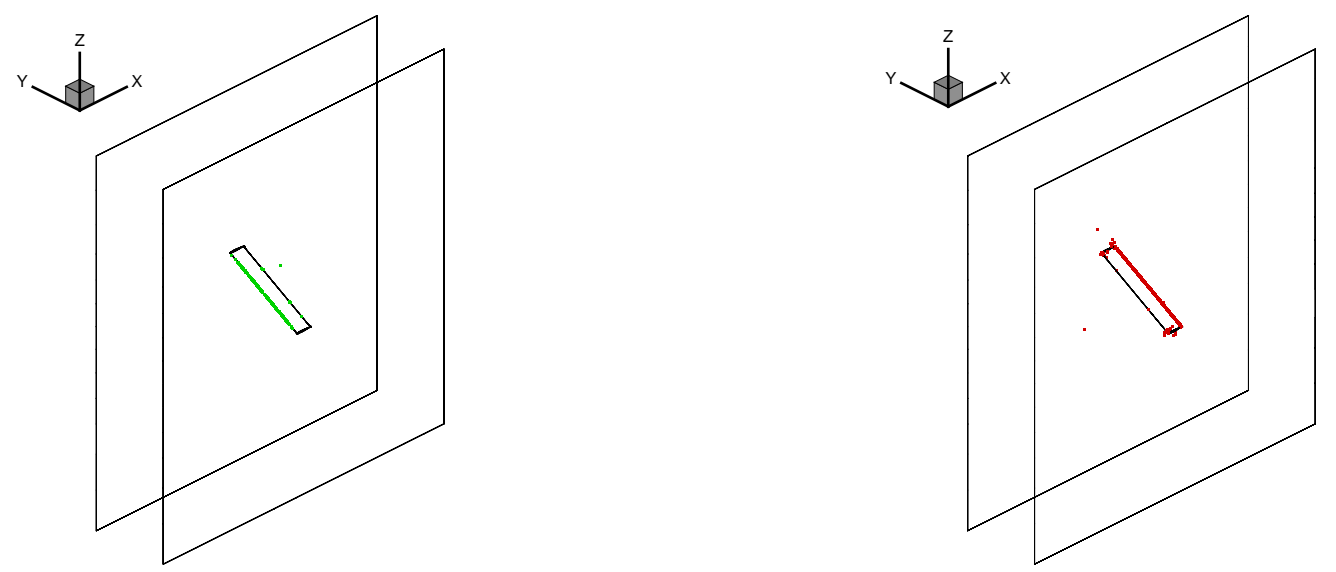

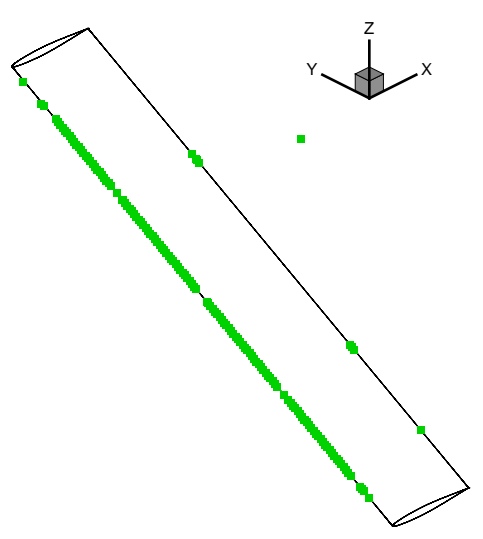

(a)

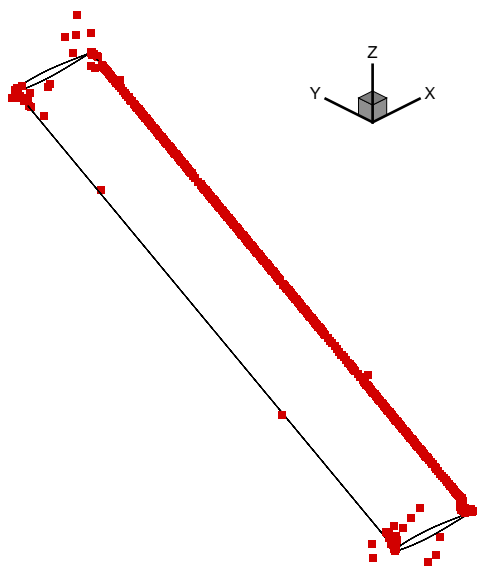

(b)

Figure 6.15: Location of the slivers in the grid for a strut inclined $60^{\circ}$ with respect to two flat walls (a) Inviscid grid (143 slivers): entire computational domain and close-up view of the strut (b) Viscous grid (5,678 slivers): entire computational domain and close-up view of the strut 


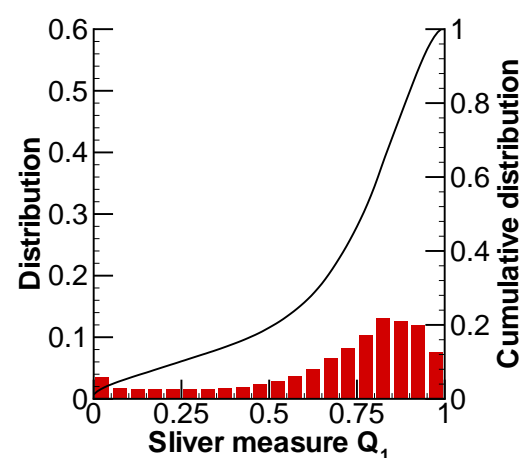

(a)

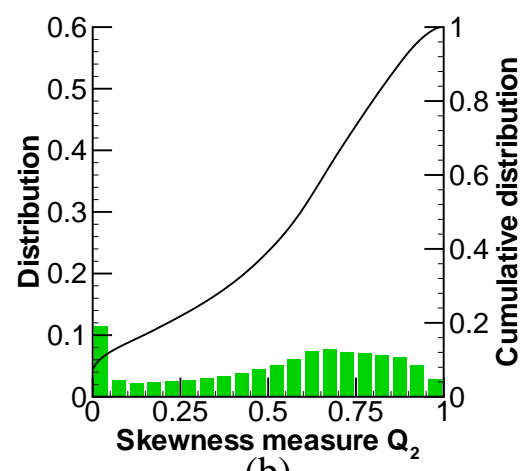

(b)

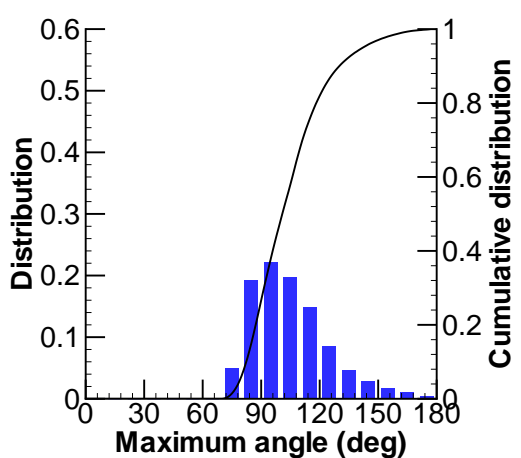

(c)

Figure 6.16: Distribution and cumulative distribution of the grid quality criteria for the inviscid grid (a) Sliver quality measure $Q_{1}$ (b) Skewness quality measure $Q_{2}$ (c) Maximum angle

fact that nearly $10 \%$ of the cells have a value of $Q_{1}$ between 0.75 and 0.80 . The solid curve is the cumulative distribution of the cells. That curve reveals that a little more than $46 \%$ of the cells have a value of $Q_{1}$ between 0 and 0.75 . The plot for the maximum angle within each cell works in the same fashion except that the distribution of the maximum angle is provided in 10-degree increments.

From the plots for $Q_{1}$ and the maximum angle presented in Figure 6.16, we can conclude that there are few potential slivers in the inviscid grid. Nearly $4 \%$ of the cells have a value of $Q_{1}$ between 0 and 0.05. But, this number becomes very small when we look at the interior angle: less than $0.4 \%$ of the cells have a maximum interior angle between 170 and 180 degrees. The majority of cells have values of $Q_{1}$ of at least 0.5 and an interior angle between 80 and 130 degrees. If we look at the skewness measure $Q_{2}$, the chart shows that almost $12 \%$ of the cells are very skewed with $0<Q_{2}<0.05$. This is due to the stretching we employ along the strut spanwise direction.

If we now consider the plot for the viscous grid statistics in Figure 6.17, there is a dramatic difference with the charts for the inviscid grid: a proportion of cells equivalent to $57 \%$ of the cells have a value of the skewness factor $Q_{2}$ between 0 and 0.05 . This should not come as a surprise since the cells generated within the viscous layers are highly stretched. And we employ stretching along the strut span as well. For the sliver quality measure, $48 \%$ of the total number of cells could be considered as slivers with $0<Q_{1}<0.05$. However, the bar chart for the maximum interior angle reveals that less than $2 \%$ of the cells have very large 


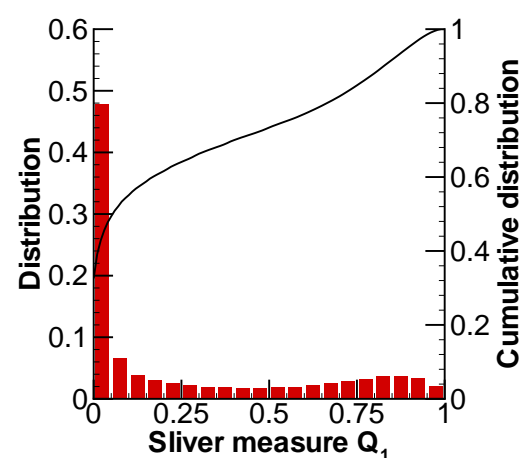

(a)

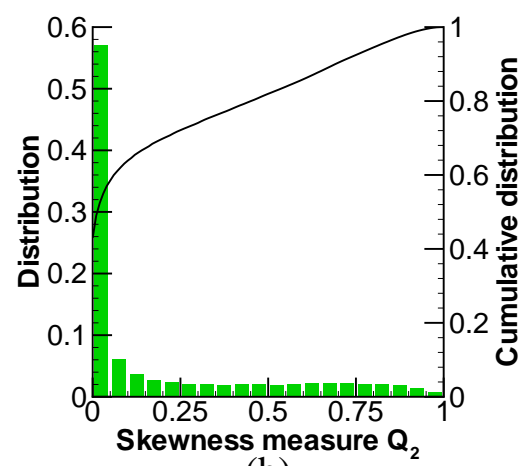

(b)

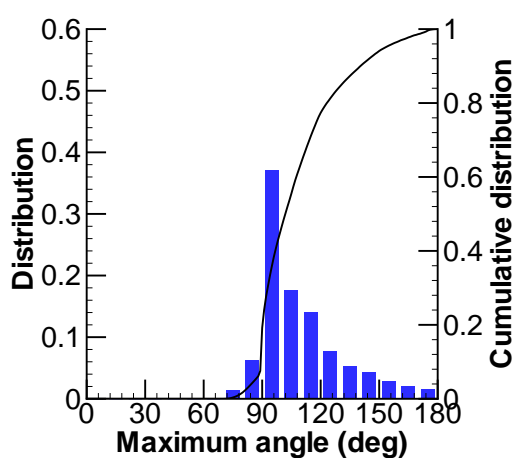

(c)

Figure 6.17: Distribution and cumulative distribution of the grid quality criteria for the viscous grid (a) Sliver quality measure $Q_{1}$ (b) Skewness quality measure $Q_{2}$ (c) Maximum angle

interior angles between 170 and 180 degrees. A large number of cells have angles around 90 degrees. This can be explained by the fact that the viscous layers are generated as close as possible to a direction perpendicular to the body surface.

One question that arises from this discussion is whether or not it is possible to do something about the slivers. Several options are available. One of them consists in removing those cells from the mesh and inserting new points in the field. This avenue is not successful with VGRIDns. The grid generation scheme is designed in such a way that even if the slivers are removed, new ones are introduced when the grid is remeshed. In many instances, more bad cells are created than there were in the original grid prior to remeshing. Another alternative consists in combining those slivers with the neighboring cells [108]. Prismatic cells are created that way. One drawback of that approach is that the flow solver has to be modified in order to accomodate for prisms in addition to the tetrahedra in the flowfield.

The method employed in this work is due to Anderson [108]. In calculating the residual and the Jacobian, the contributions of the viscous terms were neglected for the cells that have a maximum interior angle greater or equal to 178 degrees. This results in a numerical scheme that is not corrupted by the slivers and converges steadily without any problem. Since there are few slivers compared to the total number of cells in the mesh, the CFD solution is not really affected. The aerodynamic coefficients and the pressure distributions are consistent with the results obtained with the original flow solver.

In this section, the advancing-layers/advancing-front methodology implemented in the 
code VGRIDns was shown to be prone to the formation of slivers in the field. This problem occurs also for the Delaunay triangulation [121] and for the advancing-front/local-reconnection method $[99,122]$ for three-dimensional applications.

The Delaunay triangulation has to be constrained in order to preserve the surface integrity of the body [121]. Otherwise, grid points will be inserted inside the geometry and the surface triangulation will not correspond to the original geometry. The constraint imposed on the Delaunay triangulation leads to the creation of numerous slivers in the field. If the formation of slivers is prevented during the grid generation process, Baker [121] notes that the overall grid quality of the final mesh is usually worse. The best approach is to post-process the mesh in two steps. The first step consists in identifying the slivers and applying an edge-swapping technique to remove as many slivers as possible. Swapping the edges of the tetrahedral elements changes the connectivity between the existing nodes. When applied in the vicinity of a sliver, this method often gets rid of it. However, to remove the remaining slivers, a second step is required in which the grid is smoothed. The grid points that are not on the boundaries are moved in order to achieve a better grid quality. The two steps presented above can be combined in an iterative process as well.

For three-dimensional grids obtained with the advancing-front/advancing-layers approach, Marcum $[99,122]$ reports the formation of sliver elements in regions where high-aspect-ratio tetrahedra are present. This is true for viscous regions where highly stretched cells are employed. To solve the problem [122], new points are inserted in the field and connected to the existing nodes. The high-aspect ratio elements are then combined to form 6-node hexahedrons in viscous regions or 5-node pentahedrons where the viscous and inviscid regions meet. As mentioned before, this approach has the disadvantage of requiring the flow solver to handle those new elements in addition to the regular tetrahedral cells. 


\section{Chapter 7}

\section{Conclusion}

In the context of the development of a transonic strut-braced wing aircraft, the minimization of the interference drag is a key to the success of the concept. It is also a topic of primary importance in the design of wing-pylon and wing-fuselage combinations. This dissertation presents the application of CFD tools to the study of some of the parameters influencing the interference drag in the transonic speed range.

The inviscid analysis of a strut-braced wing airplane with the flow solver USM3D highlighted some important trends about the design of the strut. The MDO tools provided the planform of the airplane, but not the airfoil sections nor the twist distribution to be used for the wing and the strut. Hence, the airfoils were chosen and the wing was designed with a twist distribution which minimizes the induced drag. After validating the flow solver with the ONERA M6 wing test case, USM3D was applied to the clean wing of the strut-braced wing design. The lift distribution on the wing was close to elliptic. The pressure distributions at several span stations showed regions of constant pressure on most of the chord and the presence of a shock at the trailing edge.

The addition of the strut to the clean wing allowed an evaluation of the benefits of employing an arch-shaped strut as opposed to a straight strut. The arch-shaped strut has the advantage of increasing the distance between the wing and the strut, thus alleviating the channel effect that occurs near the junction of the wing with the strut. Also, it allows the strut to intersect the wing perpendicularly, hence minimizing the strong interference effects of small junction angles. The analysis of configurations with varying arch radius showed a significant drag reduction when the arch radius is increased. When the drag of the clean wing 
is subtracted from the total drag of the wing-strut combination, the resulting interference drag decreases significantly with an increase of the radius. An equation was fitted to the interference drag results for use in MDO studies. However, the expression developed for the interference drag doesn't take into account the effect of the thickness of the strut, the Reynolds number of the flow, or the intersection angle between the wing and the strut.

In order to evaluate the effect of these variables on the interference drag, the more general case of a strut at an angle between parallel walls was analyzed with both Euler and Navier-Stokes formulations. The approach was validated successfully with experimental data for the case of a wing perpendicular to the walls of a wind tunnel. Calculations were performed for symmetrical struts of the NACA 64A- family at Mach 0.85 for combinations of strut thicknesses $(t / c=5 \%$ and $7.5 \%)$, cruise Reynolds numbers $\left(R e_{c}=5.3 \times 10^{6}\right.$ and $\left.10.6 \times 10^{6}\right)$, and strut-wall angles $\left(\phi=30^{\circ}, 60^{\circ}\right.$, and $\left.90^{\circ}\right)$. Observations made based on the pressure distributions at several cross sections along the strut span and streamline patterns some distance away from the side wall surface showed the strong disturbance produced by the presence of the wall on the flowfield, causing in some instance the flow to separate. The viscous forces tended to alleviate the strength of the shock induced near the junction of the strut with the wall compared to predictions based on the inviscid, Euler equations. For the thicker strut perpendicular to the wall, the presence of the neighboring viscous side walls created favorable interference. The disruption of the flowfield due to the influence of the side wall was stronger for the thicker strut section than for the thinner one. The strut-wall angle exhibited a strong correlation with the drag penalty as well. The effect of inclining the strut towards the wall increased the drag by a large factor compared to the case where the strut is perpendicular to the wall. A response surface for the interference drag coefficient was developed to take into account all these effects and help in the design of junctions.

Some of the challenges and weaknesses of flow solutions on unstructured grids for problems of this type were presented. To prevent the formation of very skewed cells and voids at the wing trailing edge, alternatives were presented to close the trailing edge of airfoil sections. The results obtained with the midpoint method, the linear displacement of the upper and lower airfoil surfaces and the extrapolation of the surfaces at the trailing edge compared well with the ones computed for the original airfoil shape. Issues related to grid convergence highlighted the difficulty of obtaining finer grids from a baseline grid in a consistent fashion. The small spacing at the airfoil leading edge had a significant impact on the computed drag coefficient. A fine mesh near the trailing edge prevented the appearance of a 
peculiar variation in the pressure distribution in that region. The difficulties in obtaining a complete three-dimensional unstructured grid were explained with emphasis on surface grid crossing, memory problems, and machine precision. The presence of slivers in tetrahedral grids was shown to have a huge impact on the convergence of the flow solution. A method to circumvent the problem was described, in which the erroneous contributions of the slivers were not taken into account by the flow solver.

\section{Future Work}

There are some interesting possibilities for future work in the area presented in this dissertation:

- For the strut-wall configurations presented in Chapter 5 , the effect of the angle of attack and the sweep angle on the value of the interference drag could be investigated. On the strut-braced wing airplane, the strut is designed to carry negligible aerodynamic load along its span, but maneuvers and wind gusts can cause the flow to have an incidence angle on the strut. The consideration of that parameter would provide a better understanding of how dramatic its influence can be on the flow near the junction and on the interference drag penalty in particular. The sweep of the strut was determined to have an important effect in subsonic flow by Hoerner [24]. In transonic flow, it would provide some relief for the strut in a fashion similar to employing a swept wing on a transonic transport aircraft.

- The range of applicability of the response surface developed for the interference drag of strut-wall arrangements could be broadened by the analysis of thicker strut sections and for a wider range of Reynolds numbers than the one considered for the application to the strut-braced wing transonic transport in Chapter 5 .

- The strut-braced wing design in Chapter 4 was analyzed by solving the Euler equations. However, in light of the Navier-Stokes results obtained for a series of strut-wall combinations, it appears that the viscosity has a positive effect on the pressure drag and the strength of the shocks near the junction. The application of a Reynolds-averaged Navier-Stokes flow solver to the analysis of a complete strut-braced wing design would show the impact on the interference drag and on the flow near the junction. 
- The area ruling of the wing-strut combination would surely show a reduction in the pressure drag of the strut-braced wing. Also, in the vicinity of the junction, the application of shape optimization subject to the aircraft manufacturer's constraints would be beneficial to alleviate some of the strong interference effects between the wing and the strut.

- The aerodynamic performance of the airplane could potentially be improved with the use of tip-mounted engines because the wing tip vortex is swallowed inside the engine. The flow solver USM3D has the capability of modeling aircraft engines. A study of the strut-braced wing including the propulsive effects could be valuable in assessing the real impact of having the engines located at the wing tip.

The evaluation of all these effects takes time and money. However, the best way to do that at once is to actually build such a strut-braced wing airplane and fly it. Possibilities loom in the near future and it is hoped that they will materialize. To revolutionize the field of aeronautics, the strut-braced wing aircraft is the perfect pioneer. 


\section{Bibliography}

[1] Grasmeyer, J. M., "Multidisciplinary Design Optimization of a Transonic Strut-Braced Wing Aircraft", AIAA Paper 99-0010, 1999.

[2] Gern, F. H., Gundlach, J. F., Ko, A., Naghshineh-pour, A. H., Sulaeman, E., Tétrault, P.-A., Grossman, B., Kapania, R. K., Mason, W. H., Schetz, J. A., and Haftka, R. T., "Multidisciplinary Design Optimization of a Transonic Commercial Transport with a Strut-Braced Wing", WAC Paper 1999-01-5621, 1999 World Aviation Congress, San Francisco, CA, Oct. 19-21, 1999.

[3] Gundlach, J. F., Gern, F. H., Tétrault, P.-A., Naghshineh-pour, A. H., Ko, A., Schetz, J. A., Mason, W. H., Grossman, B., Kapania, R. K., and Haftka, R. T., "Multidisciplinary Optimization of a Strut-Braced Wing Transonic Transport", AIAA Paper 2000-0420, Jan. 2000.

[4] Technical information obtained from the web site of The Boeing Company at http://www.boeing.com/commercial/747-400/product.html.

[5] McMasters, J. H. and Kroo, I. M., "Advanced Configurations for Very Large Transport Airplanes", AIAA Paper 98-0439, 1998.

[6] Potsdam, M. A., Page, M. A., and Liebeck, R. H., "Blended Wing Body Analysis and Design", AIAA Paper 97-2317, 1997.

[7] Spearman, M. L. and Feigh, K. M., "An Airplane Configuration with an Inboard Wing Mounted Between Twin Fuselages", AIAA Paper 98-0440, 1998.

[8] Wolkovitch, J., "The Joined Wing: An Overview", Journal of Aircraft, Vol. 23, No. 3, 1986, pp. 161-178. 
[9] Gallman, J. W., Smith, S. C., and Kroo, I. M., "Optimization of Joined Wing Aircraft", Journal of Aircraft, Vol. 30, No. 6, 1993, pp. 897-905.

[10] Perkins, J. N., Cheatwood, F. M., Vess, R. J., and Wahls, R. A., "The Design and Testing of Several Joined Wing RPV's", AIAA Paper 85-0275, 1985.

[11] Smith, S. C., Cliff, S. E., and Kroo, I. M., "The Design of a Joined-Wing Flight Demonstrator Aircraft", AIAA Paper 87-2930, 1987.

[12] Gallman, J. W. and Kroo, I. M., "Structural Optimization for Joined-Wing Synthesis", Journal of Aircraft, Vol. 33, No. 1, 1996, pp. 214-223.

[13] Pfenninger, W., Design Considerations of Large Subsonic Long Range Transport Airplanes with Low Drag Boundary Layer Suction, Rep. No. NAI-58-529 (BLC-111), Northrop Corp., (Available from DTIC as AD821 759), 1958.

[14] Jobe, C. E., Kulfan, R. M., and Vachal, J. D., "Wing Planforms for Large Military Transports", AIAA Paper 78-1470, 1978.

[15] Ahmed, A. and Khan, M. J., "Effect of Sweep on Wing-Body Juncture Flow", AIAA Paper 95-0868, 1995.

[16] Barber, T. J., "An Investigation of Strut-Wall Intersection Losses", Journal of Aircraft, Vol. 15, No. 10, 1978, pp. 676-681.

[17] Burke, R. W., "Computation of Turbulent Incompressible Wing-Body Junction Flow", AIAA Paper 89-0279, 1989.

[18] Chang, P. S. and Gessner, F. B., "Experimental Investigation of Flow About a StrutEndwall Configuration", AIAA Journal, Vol. 29, No. 12, 1991, pp. 2105-2114.

[19] Chen, C.-L.. and Hung, C. M.., "Numerical Study of Juncture Flows", AIAA Journal, Vol. 30, No. 7, 1992, pp. 1800-1807.

[20] Devenport, W. J. and Simpson, R. L., "Time-Dependent and Time-Averaged Turbulence Structure Near the Nose of a Wing-Body Junction", Journal of Fluid Mechanics, Vol. 210, 1990, pp. 23-55. 
[21] Devenport, W. J., Agarwal, N. K., Dewitz, M. B., and Simpson, R. L., "Effects of a Fillet on the Flow Past a Wing-Body Junction", AIAA Journal, Vol. 28, No. 12, 1990, pp. 2017-2024.

[22] Devenport, W. J., Simpson, R. L., Dewitz, M. B., and Agarwal, N. K., "Effects of a Leading-Edge Fillet on the Flow Past an Appendage-Body Junction", AIAA Journal, Vol. 30, No. 9, 1992, pp. 2177-2183.

[23] Fleming, J. L., Simpson, R. L., and Devenport, W. J., "An Experimental Study of a Turbulent Wing-Body Junction and Wake Flow", AIAA Paper 92-0434, 1992.

[24] Hoerner, S. F., "Interference Drag", in Fluid-Dynamic Drag: Practical Information on Aerodynamic Drag and Hydrodynamic Resistance, pp. 8-1-8-20, published by S.F. Hoerner, Midland Park, N.J., 1965.

[25] Kaykayoglu, C. R., "Application of the Vortex Lattice CFD Method to Obtaining Unsteady Aerodynamic Predictions About Wing/Fuselage/Pylon/Store Configuration Including Store Separation", AIAA Paper 96-0168, 1996.

[26] Khan, M. J. and Ahmed, A., "Turbulence Measurements in Wing-Body Juncture Flow", AIAA Paper 96-0413, 1996.

[27] Krautheim, S., Miller, K. A., Campbell, B., Collicott, S. H., and Sullivan, J. P., "High Reynolds Number Experiments on Bypass Duct and Strut Flows", AIAA Paper 97$3001,1997$.

[28] Kubendran, L. R., McMahon, H., and Hubbartt, J., "Interference Drag in a Simulated Wing-Fuselage Junction", NASA CR-3811, 1984.

[29] Kubendran, L. R. and Harvey, W. D., "Juncture Flow Control Using Leading-Edge Fillets", AIAA Paper 85-4097, 1985.

[30] Kubendran, L. R., McMahon, H. M., and Hubbartt, J. E., "Turbulent Flow Around a Wing/Fuselage-Type Juncture", AIAA Journal, Vol. 24, No. 9, 1986, pp. 1447-1452.

[31] Maughmer, M. D., "An Experimental Investigation of Wing/Fuselage Integration Geometries", AIAA Paper 87-2937, 1987. 
[32] Ölçmen, M. S. and Simpson, R. L., "Influence of Wing Shapes on Surface PressureFluctuations at Wing-Body Junctions", AIAA Journal, Vol. 32, No. 1, 1994, pp. 6-15.

[33] Ölçmen, M. S. and Simpson, R. L., "Experimental Transport-Rate Budgets in Complex 3-D Turbulent Flow Near a Wing/Body Junction", AIAA Paper 96-2035, 1996.

[34] Ölçmen, S. and Simpson, R. L., "Some Features of a Turbulent Wing-Body Junction Vortical Flow", AIAA Paper 97-0651, 1997.

[35] Özcan, O. and Ölçmen, M. S., "Measurements of Turbulent Flow Behind a Wing-Body Junction", AIAA Journal, Vol. 26, No.4, 1988, pp. 494-496.

[36] Paul, B. P. and Carlson, L. A., "Analysis of Junction Flowfields Using the Incompressible Navier-Stokes Equations", AIAA Paper 92-0519, 1992.

[37] Pierce, F. J. and Nath, S. K., "Interference Drag of a Turbulent Junction Vortex", Journal of Fluids Engineering, Vol. 112, No. 4, pp. 441-446.

[38] Roach, P. E. and Turner, J. T., "Secondary Loss Generation by Gas Turbine Support Struts", International Journal of Heat and Fluid Flow, Vol. 6, No. 2, 1985, pp. 79-88.

[39] Shabaka, I. M. M. A. and Bradshaw, P., "Turbulent Flow Measurements in an Idealized Wing/Body Junction", AIAA Journal, Vol. 19, No. 2, 1981, pp. 131-132.

[40] Thwaites, B., editor, Fluid Motion Memoirs - Incompressible Aerodynamics, chapter 10, Oxford University Press, 1960.

[41] Abdulla-Altaii, A. K. and Raj, R. R., "Three-Dimensional Flow Structure in the Wake of a Wing-Body Juncture", AIAA Paper 94-2289, 1994.

[42] Slooff, J. W., "Computational Methods for Subsonic and Transonic Aerodynamic Design", in Special Course on Subsonic/Transonic Aerodynamic Interference for Aircraft, chapter 3, AGARD Report No. 712, 1983.

[43] Agrawal, S., Creasman, S. F., and Lowrie, R. B., "Evaluation of Euler Solvers for Transonic Wing-Fuselage Geometries", Journal of Aircraft, Vol. 28, No. 12, 1991, pp. 885-891. 
[44] Arabshahi, A. and Whitfield, D. L., "A Multiblock Approach to Solving the ThreeDimensional Unsteady Euler Equations about a Wing-Pylon-Store Configuration", AIAA Paper 89-3401, 1989.

[45] Baxendale, A. J., "Application of a Multiblock CFD System to Obtaining Flowfield Predictions About Wing Body Pylon Store Configurations", in Proceedings of the 17th ICAS Congress, Stockholm, 1990.

[46] Bartelheimer, W., Horstman, K. H., and Puffert-Meissner, W., "2-D Airfoil Tests Including Side Wall Boundary Layer Measurements", in A Selection of Experimental Test Cases for the Validation of CFD Codes, AGARD Advisory Report No. 303, 1994.

[47] Benek, J. A., Donegan, T. L., and Suhs, N. E., "Extended Chimera Grid Embedding Cheme with Application to Viscous Flows", AIAA Paper 8\%-1126, 1987.

[48] Carlson, J. R. and Lamb, M., "Integration Effects of Pylon Geometry and Rearward Mounted Nacelles for a High-Wing Transport", AIAA Paper 8\%-1920, 1987.

[49] Chandrasekaran, B., "Computation and Comparison of the Installation Effects of Compression Pylons for a High Wing Transport", AIAA Paper 88-0004, 1988.

[50] Chapman, G. T. and Bonness, W. K., "Multibody Interference at Transonic Mach Numbers", AIAA Paper 92-0651, 1992.

[51] Chesser, B. L., Jr.., "Analysis of an F-15E with Modified Weapons Pylon Using a Computational Fluid Dynamics Embedded Grid Method", AIAA Paper 95-1807, 1995.

[52] Cottrell, C. J. and Lijewski, L. E., "Finned, Multi-Body Aerodynamic Interference at Transonic Mach Numbers", Journal of Aircraft, Vol. 25, No. 9, 1988, pp. 827-834.

[53] Cottrell, C. J., Martinez, A., and Chapman, G. T., "Study of Multibody Aerodynamic Interference at Transonic Mach Numbers", AIAA Journal, Vol. 26, No. 5, 1988, pp. 553-560.

[54] Deese, J. E., Agarwal, R. K., and Underwood, R. R., "Numerical Solutions of the Euler Equations for Complex Three-Dimensional Aerodynamic Configurations", AIAA Paper 84-2399, 1984. 
[55] Deese, J. E. and Agarwal, R. K., "Navier-Stokes Calculations of Transonic Viscous Flow About Wing-Body Configurations", Journal of Aircraft, Vol. 25, No. 12, 1988, pp. $1106-1112$.

[56] Ecer, A., Spyropoulos, J. T., Rout, R. K., and Badesha, S., "Block-Structured Solution of Transonic Flows Around Wing/Pylon/Nacelle Configurations", AIAA Paper 89$2940,1989$.

[57] Fox, J. H., Donegan, T. L., Jacocks, J. L., and Nichols, R. H., "Computed Euler Flowfield for a Transonic Aircraft with Stores", Journal of Aircraft, Vol. 28, No. 6, 1991, pp. 389-396.

[58] Fujii, K. and Obayashi, S., "Navier-Stokes Simulations of Transonic Flows over a Wing-Fuselage Combination", AIAA Journal, Vol. 25, No. 12, 1987, pp. 1587-1596.

[59] Gea, L. M., Chyu, W. J., Stortz, M. W., and Chow, C. Y., "Juncture Flow Improvement for Wing/Pylon Configurations by Using CFD Methodology", AIAA Paper 93-0522, 1993.

[60] Jordan, J. K., "Computational Investigation of Predicted Store Loads in Mutual Interference Flow Fields", AIAA Paper 92-4570, 1992.

[61] Kao, T. J., Su, T. Y., and Yu, N. J., "Navier-Stokes Calculations for Transport WingBody Configurations with Nacelles and Struts", AIAA Paper 93-2945, 1993.

[62] Lijewski, L. E., "Transonic Euler Solutions on Mutually Interfering Finned Bodies", AIAA Journal, Vol. 28, No. 6, 1990, pp. 982-988.

[63] Lijewski, L. E., "Transonic Euler Solutions of a Wing-Pylon-Finned Body Configuration Using Blocked and Overlapping Grid Schemes", AIAA Paper 91-2854, 1991.

[64] Lijewski, L. E., "Transonic Mutual Interference of Wing-Pylon-Multiple Body Configurations Using an Overlapping Grid Scheme", AIAA Paper 93-3023, 1993.

[65] Lijewski, L. E. and Suhs, N. E., "Time-Accurate Computational Fluid-Dynamics Approach to Transonic Store Separation Trajectory Prediction", Journal of Aircraft, Vol. 31, No. 4, 1994, pp. 886-891. 
[66] Lord, W. K. and Zysman, S. H., "VSAERO Analysis of a Wing/Pylon/Nacelle Configuration", AIAA Paper 86-1523, 1986.

[67] Lynch, P. L. and Rizk, M. H., "Thin-Layer Navier-Stokes Solutions for Transonic Multi-Body Interference", AIAA Paper 91-0071, 1991.

[68] Madson, M., Moyer, S., and Cenko, A., "TRANAIR Computations of the Flow About a Generic Wing/Pylon/Finned-Store Configuration", AIAA Paper 94-0155, 1994.

[69] Meakin, R. L., "Computations of the Unsteady Flow About a Generic Wing/Pylon/Finned-Store Configuration", AIAA Paper 92-4568, 1992.

[70] Naik, D., "Innovative Pylon Concepts for Engine-Airframe Integration for Transonic Transports", AIAA Paper 89-1819, 1989.

[71] Naik, D. A., Ingraldi, A. M., and Pendergraft, O. C., "Experimental Study of Pylon Cross Sections for a Subsonic Transport Airplane", Journal of Aircraft, Vol. 30, No. 5, 1993, pp. 676-681.

[72] Newman, J. C. and Baysal, O., "Transonic Solutions of a Wing/Pylon/Finned Store Using Hybrid Domain Decomposition", AIAA Paper 92-4571, 1992.

[73] Parikh, P., Pirzadeh, S., and Frink, N. T., "Unstructured Grid Solutions to a Wing/Pylon/Store Configuration", Journal of Aircraft, Vol. 31, No. 6, 1994, pp. 12911296.

[74] Potsdam, M. A., Intemann, G. A., Frink, N. T., Campbell, R. L., Smith, L. A., and Pirzadeh, S., "Wing/Pylon Fillet Design Using Unstructured Mesh Euler Solvers", AIAA Paper 93-3500, 1993.

[75] Rosen, B. S., Method to Predict External Store Carriage Characteristics at Transonic Speeds, NASA CR-4170, August 1988.

[76] Shankar, V. and Malmuth, N. D., "Computational and Simplified Analytical Treatment of Transonic Wing-Fuselage-Pylon-Store Interactions", AIAA Paper 80-0127, 1980.

[77] Sundaram, P., Chaudhuri, S. N., and Wu, J. M., "Computation of Transonic Flow Field Over Wing-Body-Pylon-Store Combinations", AIAA Paper 83-1852, 1983. 
[78] Suhs, N. E., "Computational Estimations of Strut Support Interference at Transonic Mach Numbers", AIAA Paper 85-5018, 1985.

[79] Tu, Y., Noack, R. W., and Bishop, D., "Three-Dimensional Euler Solutions on WingPylon-Store Configuration with Unstructured Tetrahedral Meshes", AIAA Paper 92$4574,1992$.

[80] Vatsa, V. N. and Wedan, B. W., "Navier-Stokes Solutions for Transonic Flow Over a Wing Mounted in a Tunnel", AIAA Paper 88-0102, 1988.

[81] Chen, P. C. and Liu, D. D., "Unsteady Supersonic Computations of Arbitrary WingBody Configurations Including External Stores", Journal of Aircraft, Vol. 27, No. 2, 1990, pp. 108-116.

[82] Hung, C.-M. and Buning, P. G., "Simulation of Blunt-Fin-Induced Shock Wave and Turbulent Boundary-Layer Interaction", AIAA Paper 84-0457, 1984.

[83] Knight, D. D., Badekas, D., Horstman, C. C., and Settles, G. S., "Quasiconical Flowfield Structure of the Three-Dimensional Single Fin Interaction", AIAA Journal, Vol. 30, No. 12, 1992, pp. 2809-2816.

[84] Kulfan, R. M., "Application of Favorable Aerodynamic Interference to Supersonic Airplane Design", SAE Paper 901988, 1990.

[85] Lakshmanan, B. and Tiwari, S. N., "Study of Supersonic Intersection Flowfield at Modified Wing-Body Junctions", AIAA Journal, Vol. 31, No. 5, 1993, pp. 877-883.

[86] Lakshmanan, B. and Tiwari, S. N., "Investigation of Three-Dimensional Separation at Wing/Body Junctions in Supersonic Flows", Journal of Aircraft, Vol. 31, No. 1, 1994, pp. $64-71$.

[87] McMaster, D. L. and Shang, J. S., "A Numerical Study of Three-Dimensional Separated Flows Around a Sweptback Blunt Fin", AIAA Paper 88-0125, 1988.

[88] Philpott, D. R. and Zhao, J. Z., "Interference Effects Between Spherically Blunted Cylinders at $\mathrm{M}=2.5$ and 1.5", AIAA Paper 84-2098, 1984.

[89] Pritulo, T. M., Gubanov, A. A., and Voevodenko, N. V., "Favorable Interference of Optimized Wing-Body Combination with Inlets at Supersonic Speeds", AIAA Paper 95-3946, 1995. 
[90] Williams, K. E., Gessner, F. B., and Harloff, G. J., "Experimental and Numerical Investigation of Supersonic Turbulent Flow in an Annular Duct", AIAA Journal, Vol. 32, No. 7, 1994, pp. 1528-1531.

[91] Harloff, G. J., Williams, K. E., and Gessner, F. B., "Numerical Investigation of Supersonic Annular Flow About Struts of Various Thicknesses", AIAA Journal, Vol. 33, No. 3, 1995, pp. 562-564.

[92] Williams, K. E., Harloff, G. J., and Gessner, F. B., "Investigation of Supersonic Flow About Strut Endwall Intersections in an Annular Duct", AIAA Journal, Vol. 33, No. 4, 1995, pp. 586-594.

[93] Williams, K. E., Harloff, G. J., and Gessner, F. B., "Effect of Strut Sweep on Supersonic Flow About Strut/Endwall Intersections", AIAA Paper 95-0100, 1995.

[94] Zemsch, S. and Degrez, G., "Wing/Fuselage Interferences in Supersonic and Hypersonic Flow", AIAA Paper 93-5140, 1993.

[95] Frink, N. T., "Upwind Scheme for Solving the Euler Equations on Unstructured Tetrahedral Meshes", AIAA Journal, Vol. 30, No. 1, 1992, pp. 70-77.

[96] Thompson, J. F., Soni, B. K., and Weatherill, N. P., editors, Handbook of Grid Generation, CRC Press, 1999.

[97] Mavriplis, D. J., "Unstructured Grid Techniques", Annual Review of Fluid Mechanics, Vol. 29, 1997, pp. 472-514.

[98] Marcum, D. L. and Weatherill, N. P., "Unstructured Grid Generation Using Iterative Point Insertion and Local Reconnection", AIAA Journal, Vol. 33, No.9, 1995, pp. 1619 1625.

[99] Marcum, D. L., "Generation of Unstructured Grids for Viscous Flow Applications", AIAA Paper 95-0212, 1995.

[100] Pirzadeh, S., "Structured Background Grids for Generation of Unstructured Grids by Advancing-Front Method", AIAA Journal, Vol. 31, No. 2, 1993, pp. 257-265.

[101] Pirzadeh, S., "Three-Dimensional Unstructured Viscous Grids by the AdvancingLayers Method", AIAA Journal, Vol. 34, No. 1, 1996, pp. 43-49. 
[102] Samareh, J., "GridTool: A Surface Modeling and Grid Generation Tool", in Proceedings of the Workshop on Surface Modeling, Grid Generation, and Related Issues in CFD Solutions, NASA CP-3291, May 9-11, 1995.

[103] Pirzadeh, S., Private communication. Configuration Aerodynamics Branch, Mail Stop 499, 16 Victory Street, NASA Langley Research Center, Hampton, VA 23681-2199.

[104] Anderson, W. K. and Bonhaus, D. L., "An Implicit Upwind Algorithm for Computing Turbulent Flows on Unstructured Grids", Computers and Fluids, Vol. 23, No.1, 1994, pp. $1-21$.

[105] Anderson, W. K., Rausch, R. D., and Bonhaus, D. L., "Implicit/Multigrid Algorithms for Incompressible Turbulent Flows on Unstructured Grids", J. Comp. Physics, Vol. 128, 1996, pp. 391-408.

[106] Spalart, P. R. and Allmaras, S. R., "A One-Equation Turbulence Model for Aerodynamic Flows", La Recherche Aérospatiale, Vol. 1, 1994, pp. 5-21.

[107] Tannehill, J. C., Anderson, D. A., and Pletcher, R. H., Computational Fluid Mechanics and Heat Transfer - Second Edition, Taylor \& Francis, 1997.

[108] Anderson, W. K., Private communication. Computational Modeling and Simulation Branch, Mail Stop 128, 18e West Taylor Street, NASA Langley Research Center, Hampton, VA 23681-2199.

[109] Briggs, W., A Multigrid Tutorial, SIAM, 1987.

[110] Frink, N. T., Pirzadeh, S., and Parikh, P., An Unstructured-Grid Software System for Solving Complex Aerodynamic Problems, NASA CP-3291, May 1995.

[111] Schmitt, V. and Charpin, F., "Pressure Distributions on the ONERA M6-Wing at Transonic Mach Number", AGARD Advisory Report 138, May 1979.

[112] Harris, C. D., "NASA Supercritical Airfoils", NASA TP 2969, March 1990.

[113] Lamar, J. E., "A Vortex Lattice Method for the Mean Camber Shapes of Trimmed Non-Coplanar Planforms with Minimum Vortex Drag", NASA TN D-8090, 1976.

[114] Mason, W. H., "Wing-Canard Aerodynamics at Transonic Speeds - Fundamental Considerations on Minimum Drag Spanloads", AIAA Paper 82-0097, 1982. 
[115] Roache, P. J., Verification and Validation in Computational Science and Engineering, chapter 5, pp. 109-112, Hermosa Publishers, 1998.

[116] JMP Statistical Discovery Software, Version 3.1, SAS Institute Inc., Cary, NC, USA, 1995.

[117] Ladson, C. L. and Brooks, C. W., Jr., "Development of a Computer Program to Obtain Ordinates for NACA 6- and 6A- Series Airfoils", NASA TM X-3069, Sept. 1974.

[118] Rivers, M. B. and Wahls, R. A., Comparison of Computational and Experimental Results for a Supercritical Airfoil, NASA TM-4601, November 1994.

[119] Gaither, A. and Marcum, D. L., SolidMesh User's Manual, National Science Foundation Engineering Research Center (ERC) for Computational Field Simulation (CFS), Mississippi State University, available at http://www.erc.msstate.edu/research/thrusts/grid/solid_mesh/.

[120] Anderson, J. D., Jr., Fundamentals of Aerodynamics, Second Edition, McGraw-Hill, 1991.

[121] Baker, T. J., "Delaunay-Voronoï Methods", in Thompson, J. F., Soni, B. K., and Weatherill, N. P., editors, Handbook of Grid Generation, chapter 16, CRC Press, 1999.

[122] Marcum, D. L., "Unstructured Grid Generation Using Automatic Point Insertion and Local Reconnection", in Thompson, J. F., Soni, B. K., and Weatherill, N. P., editors, Handbook of Grid Generation, chapter 18, CRC Press, 1999. 


\section{Vita}

Philippe-André Tétrault was born on January 11, 1973 in Montréal, Canada. He grew up in Laval with his parents, Marie-Claude and Guy-Paul, and his younger sister, Rose-Marie. In 1992, he began his Bachelor's degree at École Polytechnique de Montréal in Canada. He graduated in 1996 with a degree in Mechanical Engineering with a specialty in Aeronautical Engineering. During his studies, he obtained summer internships with the aircraft manufacturer Bombardier (formerly known as Canadair) and with CAE Electronics Ltd., the world-leading manufacturer of flight simulators. He began his M.S. and Ph.D. degrees at Virginia Tech in the Fall of 1996. He joined a team of faculty and graduate students whose main task was to investigate the concept of strut-braced wing with multidisciplinary design optimization tools. He obtained his M.S. degree in the Fall of 1998. Upon completion of his doctoral studies, he will start working for Pratt \& Whitney Canada in Longueuil, Canada as senior aerodynamicist for the group of Installations Aerodynamics and Acoustics. He will investigate the noise and aerodynamics of installed turboprop engines on aircraft. 